\title{
Access to Highly Functionalized Cyclopentenones via Diastereoselective Pauson Khand Reaction of Siloxy-Tethered 1,7-Enynes
}

Austin Gallagher, Huan Tian, Osmar A. Torres-Herrera, Shuai Yin, Anxin Xie, Daniel M. Lange, Jerica K. Wilson, Louis G. Mueller, Michael R. Gau, Patrick J. Carroll, Dionicio Martinez

Submitted date: 21/08/2019 - Posted date: 21/08/2019

Licence: CC BY-NC-ND 4.0

Citation information: Gallagher, Austin; Tian, Huan; Torres-Herrera, Osmar A.; Yin, Shuai; Xie, Anxin; Lange, Daniel M.; et al. (2019): Access to Highly Functionalized Cyclopentenones via Diastereoselective Pauson Khand Reaction of Siloxy-Tethered 1,7-Enynes. ChemRxiv. Preprint.

A diastereoselective $\mathrm{Co}_{2}(\mathrm{CO})_{8}$-mediated Pauson-Khand reaction $(\mathrm{PKR})$ of siloxy-tethered 1,7-enynes for the synthesis of cyclopentaoxasilinones has been developed. This transformation can be performed on a multi-gram scale and is characterized by broad substrate scope, functional group compatibility, and high chemo- and diastereoselectivity. Oxidation of the resulting cyclopentaoxasilinones delivers stereoenriched $\beta$-alkylated cyclopentenones, which are inaccessible by intermolecular PKRs. This research provides a practical solution to the challenges associated with the classical intermolecular PKR.

File list (2)

Si-O_PKR_manuscript_final_draft.pdf (1.29 MiB)

view on ChemRxiv • download file 


\title{
Access to Highly Functionalized Cyclopentenones via Diastereoselec- tive Pauson Khand Reaction of Siloxy-Tethered 1,7-Enynes
}

\author{
Austin G. Gallagher, ${ }^{\dagger}$ Huan Tian, ${ }^{\dagger}$ Osmar A. Torres-Herrera ${ }^{\dagger}$ Shuai Yin, ${ }^{\dagger}$ Anxin Xie, ${ }^{\dagger}$ Daniel M. \\ Lange ${ }^{\dagger \S}$ Jerica K. Wilson, ${ }^{\dagger \perp}$ Louis G. Mueller, ${ }^{\dagger \perp}$ Michael R. Gau, ${ }^{\ddagger}$ Patrick J. Carroll, ${ }^{\ddagger}$ and Dionicio Mar- \\ tinez-Solorio $*^{\dagger}$ \\ ${ }^{\dagger}$ Department of Chemistry, Drexel University, 32 South 32nd Street, Philadelphia, Pennsylvania 19104, United States \\ ${ }^{\ddagger}$ Department of Chemistry, University of Pennsylvania, 231 South 34th Street, Philadelphia, Pennsylvania 19104, United \\ States
}

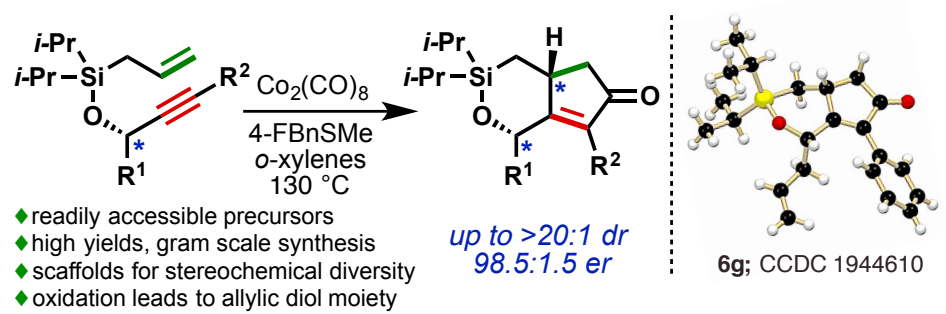

\begin{abstract}
A diastereoselective $\mathrm{Co}_{2}(\mathrm{CO})_{8}$-mediated Pauson-Khand reaction (PKR) of siloxy-tethered 1,7-enynes for the synthesis of cyclopentaoxasilinones has been developed. This transformation can be performed on a multi-gram scale and is characterized by broad substrate scope, functional group compatibility, and high chemo- and diastereoselectivity. Oxidation of the resulting cyclopentaoxasilinones delivers stereoenriched $\beta$-alkylated cyclopentenones, which are inaccessible by intermolecular PKRs. This research provides a practical solution to the challenges associated with the classical intermolecular PKR.
\end{abstract}

Stereoselective strategies for the synthesis of densely functionalized cyclopentenones are highly sought after because they enable assembly of structural components present in a broad range of biologically active natural products (Scheme 1a). ${ }^{1}$ The cobalt-mediated Pauson-Khand Reaction (PKR) exemplifies a convergent, atom-economical, ${ }^{2}$ and fundamental reaction for the synthesis of cyclopentenones. ${ }^{3}$ Since Khand and Pauson's initial discovery in $1973,{ }^{4}$ significant advances in the intermolecular PKR have been made. ${ }^{5}$ However, this reaction is generally limited to strained or symmetrical olefins due to the difficulty in controlling the alkene regio- and stereoselectivity (Scheme 1b). ${ }^{6}$ Pioneering studies by Riera and Verdaguer et al. have shown that chiral bidentate PuPhos, CamPhos, PNSO, and ThaxPhos ligands are efficient in cobalt-catalyzed asymmetric intermolecular PKRs. Notwithstanding, these studies are limited to the use of symmetrical and strained norbornadiene. ${ }^{7}$ Carreterro and co-workers reported the first example of an asymmetric intermolecular PKR with unstrained olefins employing stoichiometric cobaltalkyne complexes and $o$-(dimethylamino)phenyl vinyl sulfoxide as a chiral auxiliary. ${ }^{8}$ While intramolecular PKRs are showcased in numerous elegant total syntheses, ${ }^{9}$ the application of the intermolecular variant has been limited. ${ }^{10}$ Thus, a general stereoselective PKR strategy to access highly functionalized monocyclic cyclopentenones remains an unsolved challenge.

a) Representative bioactive cyclopentenoid natural products

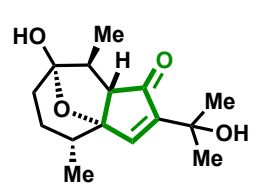

calamusin $B$

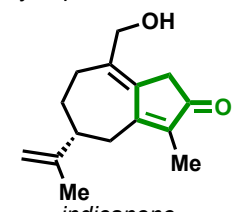

indicanone

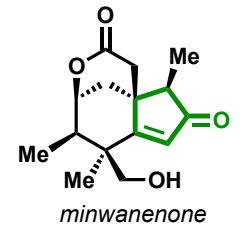

b) The Intermolecular Pauson-Khand reaction (PKR)
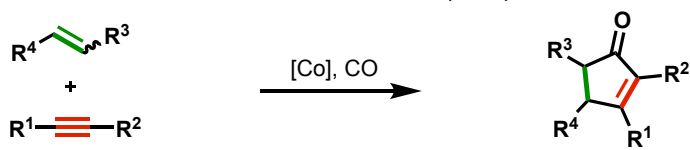

- poor reactivity/regioselectivity

- enatioselective systems underdeveloped

- restricted to symmetrical or strained alkenes

c) This research: Si-O tethered diastereoselective PKR strategy

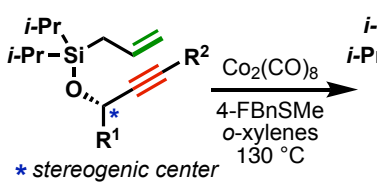

$\checkmark$ readily accesible precursors $\checkmark$ high yields, gram scale synthesis

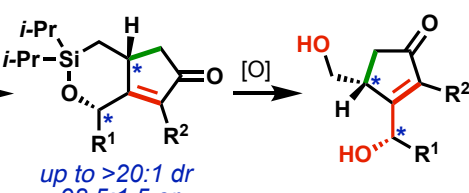
98.5:1.5 er
98:

$\checkmark$ oxidation leads to allylic diol moiety $\checkmark$ scaffolds for stereochemical diversity

Scheme 1. (a) Cyclopentanoid natural products. (b) Challenges associated with the intermolecular PKR. (c) This research: Siloxy-tethered diastereoselective PKR from propargyl alcohols. 
A tactic to enhance reactivity and control regio- and stereoselectivity is to employ a removable tether, preferably one that allows further functionalization (Scheme 1c). ${ }^{11}$ Silicon has proven a practical tethering element in a multitude of transformations. ${ }^{12}$ It is particularly useful because it serves as a convenient retron for hydroxyl groups via Tamao-Fleming oxidation, ${ }^{13 \mathrm{a}-\mathrm{f}}$ as well as a plethora of stereoselective transformations. ${ }^{13 \mathrm{~g}}$ Prior reports of $\mathrm{Si}-\mathrm{O}$ tethered PKRs suffer from unexpected by-products, low yields, and limited substrate scope. ${ }^{14 a-f}$ Brummond's group reported an example of an allenic $\mathrm{Mo}(\mathrm{CO})_{6}$-mediated $\mathrm{Si}-\mathrm{O}$ tethered $\mathrm{PKR}$ in $15-25 \%$ yields. ${ }^{14 \mathrm{~d}}$ Dobbs et al. reported four examples with diisopropyl $\mathrm{Si}-\mathrm{O}$ tethered 1,7-enynes derived from primary propargyl alcohols utilizing $\mathrm{NMO}$ promoted $\mathrm{Co}_{2}(\mathrm{CO})_{8}$-mediated PKR conditions. ${ }^{14 \mathrm{f}}$ Similarly, Porter and co-workers ${ }^{14 \mathrm{e}}$ investigated $\mathrm{Co}_{2}(\mathrm{CO})_{8}$-mediated PKRs of diphenyl $\mathrm{Si}-\mathrm{O}$ tethered 1,7enynes employing $n$-BuSMe ${ }^{15}$ as the promoter. In a single example, a secondary propargyl alcohol furnished the PKR product in low yield and diastereoselectivity $(9 \% ; 1: 1.5 \mathrm{dr})$. Inspired by these reports, we hypothesized that utilizing $\mathrm{Si}-\mathrm{O}$ tethered 1,7-enynes could lead to a highly diastereoselective PKR. ${ }^{16}$ An attractive feature of this approach is the use of enantioenriched secondary propargyl alcohols, readily accessible via a variety of well-established methods. ${ }^{17-19}$ Successful execution of such a tactic would provide otherwise difficult to access diastereoenriched $\beta$-alkylated cyclopentenones from readily accessible starting materials. (Scheme 1c). Central to our approach is the oxidative cleavage of the $\mathrm{Si}-\mathrm{C}$ bond (vide infra; 7d and 7f, Scheme 5), which unveils useful allylic and homoallylic diol moieties for further structural and stereochemical augmentation. ${ }^{20}$ In this Communication, we describe our efforts toward a cobalt-mediated diastereoselective $\mathrm{Si}-\mathrm{O}$ tethered PKR of 1,7-enynes for the synthesis of cyclopentaoxasilinones. By employing a functionalizable silicon-tether, the methodology presented herein provides a practical solution to the challenges associated with the intermolecular PKR.

Optimization studies (Table 1) commenced with an evaluation of solvent, temperature, time, concentration, and promoters in the PKR of model substrate 1a to afford cyclopentaoxasilinone 2a. Treatment with 1.05 equiv of $\mathrm{Co}_{2}(\mathrm{CO})_{8}$ with $\mathrm{N}$ oxides $^{21,22}$ at room temperature gave low yields (Table 1, Entries 1 - 2). Cyclohexylamine $\left(\mathrm{CyNH}_{2}\right)$ reported as an effective Lewis base promoter ${ }^{23}$ led to similar results $(22 \%$; Entry $3)$. We were pleased to observe that upon treatment with 1.05 equiv of $\mathrm{Co}_{2}(\mathrm{CO})_{8}, 3.50$ equiv of $n$-BuSMe in $o$-xylenes $(0.1 \mathrm{M})$ at $130^{\circ} \mathrm{C}$ for $12 \mathrm{~h}$ resulted in improved yields of $\mathbf{2 a}$ (57\%; Entry 6). Notably, reducing the reaction time from $24 \mathrm{~h}$ to $12 \mathrm{~h}$ did not negatively impact the yield (Entry $5 \mathrm{vs}$. Entry6). However, $24 \mathrm{~h}$ was required to give comparable yields of $2 \mathbf{a}$ at $110{ }^{\circ} \mathrm{C}$ in toluene (Entry 4). Based on Magnus' proposed mechanism of the PKR, ${ }^{24}$ Sugihara's report, ${ }^{15}$ and seminal mechanistic studies by the groups of Krafft, ${ }^{25 a}$ Nakamu$\mathrm{ra}^{25 \mathrm{~b}}$ Milet and Gimbert, ${ }^{25 \mathrm{c}}$ we reasoned that modulating the Lewis basicity of the thioether would impact the rate of alkene insertion and the overall rate of reaction as well as lead to improved yields. To test this hypothesis, we turned our attention to a variety of commercially available and readily accessible thioether additives. While PhSMe gave similar yields $(55 \%$; Entry 7) to $n$-BuSMe (57\%; Entry 6), BnSMe and 4OMeBnSMe led to an increase in yield of $2 \mathbf{a}$ to $65 \%$ and $64 \%$ yields, respectively (Entries 8 -9). Overall, we found 5.0 equiv of both commerciallyl available furfuryl methyl sulfide
Table 1. Optimization Studies and Discovery of a Recoverable/Reusable PKR Promotor (4-FBnSMe)

\begin{tabular}{|c|c|c|c|c|c|}
\hline Entry $^{a}$ & Solvent & Promotor & $\begin{array}{c}T \\
\left({ }^{\circ} \mathrm{C}\right)\end{array}$ & $\begin{array}{c}\text { Time } \\
\text { (h) }\end{array}$ & $\begin{array}{l}\text { Yield (\%) } \\
2 \mathrm{a}^{e}\end{array}$ \\
\hline 1 & DCE & $\mathrm{NMO}^{b}$ & 25 & 8 & 17 \\
\hline 2 & DCE & TMANO $^{b}$ & 25 & 8 & 24 \\
\hline 3 & DCE & $\mathrm{CyNH}_{2}{ }^{c}$ & 85 & 12 & 22 \\
\hline 4 & $\mathrm{PhCH}_{3}$ & $n$-BuSMe ${ }^{c}$ & 110 & 24 & 47 \\
\hline 5 & o-xylenes & $n$-BuSMe ${ }^{c}$ & 130 & 24 & 55 \\
\hline 6 & o-xylenes & $n$-BuSMe ${ }^{c}$ & 130 & 12 & 57 \\
\hline 7 & o-xylenes & $\mathrm{PhSMe}^{c}$ & 130 & 12 & 55 \\
\hline 8 & o-xylenes & $\mathrm{BnSMe}^{c}$ & 130 & 12 & 65 \\
\hline 9 & o-xylenes & 4-OMeBnSMe & 130 & 12 & 64 \\
\hline 10 & o-xylenes & furfurylSMe ${ }^{d}$ & 130 & 12 & 72 \\
\hline 11 & o-xylenes & 4-FBnSMe ${ }^{d}$ & 130 & 12 & 81 \\
\hline 12 & o-xylenes & --- & 130 & 12 & $36^{f}$ \\
\hline
\end{tabular}

${ }^{a} 1.0$ equiv of $1 \mathrm{a}, 0.33 \mathrm{mmol}$ scale $\left(0.10 \mathrm{M}\right.$ of solvent). ${ }^{b} 10$ equiv. ${ }^{c} 3.5$ equiv. ${ }^{d} 5$ equiv. ${ }^{e}$ Purified/isolated yields represent an average of 3 experiments. ${ }^{f}$ Promotor omitted.

(furfurylSMe) or 4-fluorobenzyl(methyl)sulfide (4-FBnSMe), affords $2 \mathrm{a}$ in improved yields ( $72 \%$ and $81 \%$ respectively; Entries $10-11)$. Moreover, 4-FBnSMe is conveniently prepared in one-step on a multi-gram scale, is recoverable via flash chromatography, and can be reused without loss of reactivity ( $83 \%$ average recovery; See ESI). A control experiment, in which the promotor was omitted (36\%; Entry 12), demonstrates the value of 4-FBnSMe as a new, efficient, and recoverable/reusable thioether promoter in the PKR.

With optimized conditions in hand, we initiated a systematic study of the scope and limitation $\left(\mathrm{R}^{2}\right.$; Scheme 2$)$ of the $\mathrm{Si}-\mathrm{O}$

Scheme 2. Variation of Alkynyl Substituents ${ }^{a, b, c}$
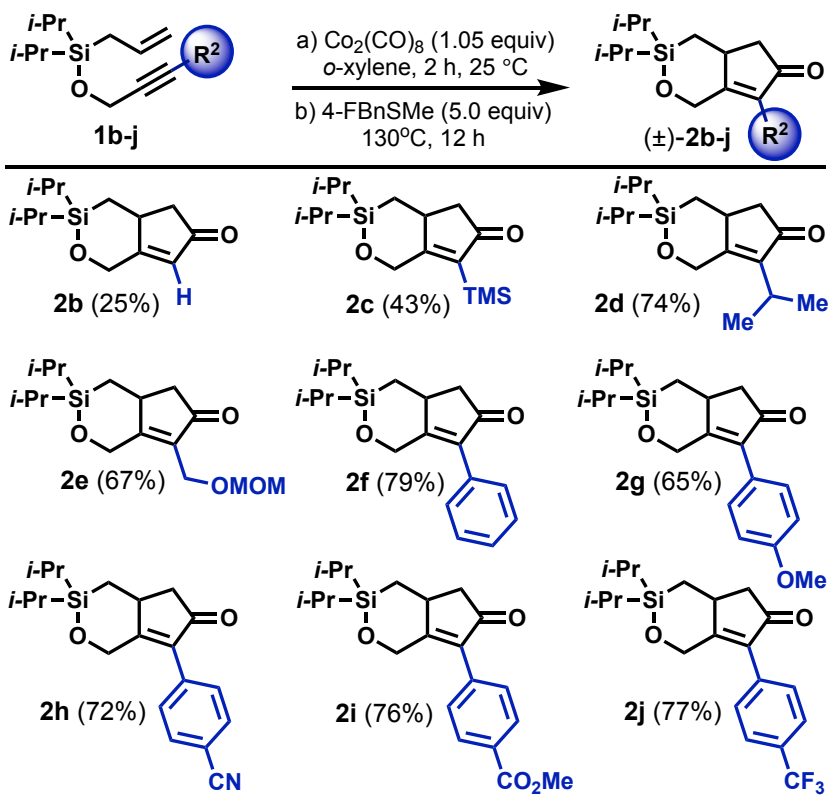

${ }^{a} 1.0$ equiv of $\mathbf{1 b}-\mathbf{j}, 0.33 \mathrm{mmol}$ scale $(0.10 \mathrm{M}) .{ }^{b}$ Purified/isolated yields represent an average of 3 experiments. ${ }^{c} 83 \%$ average recovery of 4-FBnSMe. 
tethered PKR of 1,7-enynes $\mathbf{1 b}-\mathbf{1} \mathbf{j}$ containing a variety of alkynyl substituents. Unsubstituted enyne $\mathbf{1 b}$ did not perform well under the current reaction conditions providing $\mathbf{2 b}$ in $25 \%$ yield. Trimethylsilane substituted enyne 1c gave a diminished yield of product $2 \mathrm{c}(43 \%)$, despite a $24 \mathrm{~h}$ extended reaction time. In contrast, isopropyl substituted 2d reached full conversion in $12 \mathrm{~h}$ and was isolated in 74\% yield. Methoxymethyl-protected propargyl ether 2e was furnished in $67 \%$ yield, providing an opportunity for post-PKR synthetic manipulations. Phenyl substituted $\mathbf{2 f}$ was isolated in $79 \%$ yield while the electron-rich analogue (4-OMe) gave $\mathbf{2 g}$ in a slightly diminished yield of $65 \%$. Furthermore, electron-withdrawing para-substituted arenes $\mathbf{2} \mathbf{h}-\mathbf{j}$ were obtained in good yields and demonstrate excellent functional group compatibility; $-\mathrm{CN}$ $(72 \%),-\mathrm{CO}_{2} \mathrm{Me}(76 \%),-\mathrm{CF}_{3}(77 \%)$.

Building on this initial success, we turned our attention to the development of a diastereoselective PKR from secondary propargyl alcohols (Scheme 3). Upon close examination of molecular models, we hypothesized that the sterically hindered $i$-Pr alkyl substituents of silicon would serve a dual purpose: (1) inducing a Thorpe-Ingold effect, ${ }^{26}$ thereby increasing the rate of reaction, and (2) imparting a favorable steric influence on the transition state of the cycloaddition, which would lead to improved diastereoselectivity. As delineated in Scheme 3, alkyl substituted propargyl enynes $\mathbf{5 a}-\mathbf{b}$ provided products 6a-6b in moderate selectivity (syn/anti; 6:1 and 3:1 dr, respectively) and in moderate yields (31\% and 59\%, respectively). On the other hand, phenyl substituted $\mathbf{6 c}$ was furnished in high yield with similar selectivity $(81 \% ; 5.5: 1 \mathrm{dr})$. Unsurprisingly, decomposition of enyne 5d was observed via TLC analysis upon cobalt complexation, ${ }^{27}$ and $\mathbf{6 d}$ was not detected. However, benzyl substituted enyne 5e provided the PKR product $\mathbf{6 e}$ in high yield and selectivity (88\% yield, and 13:1 dr). We also performed the reaction with this substrate on gram scale attesting to the robust nature of the reaction $(1.13 \mathrm{~g}, 3.0 \mathrm{mmol}$ of enyne 5e). Extending the carbon chain length by one methylene unit gave product 6 f in $84 \%$ yield, albeit with diminished selectivity $(4: 1 \mathrm{dr})$. In all cases, mixtures of diastereomers were readily separable via simple flash chromatography. Much to our delight, enyne $\mathbf{5 g}$ with an appended allyl group provided the product $\mathbf{6 g}$ in $87 \%$ isolated yield as a single diastereomer $(>20: 1 \mathrm{dr})$ on a 2.5-gram scale of enyne $\mathbf{5 g}$ (7.65 mmol)! Remarkably, extending the carbon chain length by two methylene groups before the terminal olefin led to a completely chemo- and diastereoselective PKR that furnished the product $6 \mathbf{i}$ in excellent yield $(89 \%)$. The alternative PKR product, where the terminal olefin engages with the cobaltacycle for the cyclization event instead of the allyl silane, is not detected. By contrast, omitting the olefinic moiety from the starting material furnishes products $\mathbf{6 h}$ and $\mathbf{6 j}$ in good yields (70\% and 68\%, respectively), however with diminished selectivity (3.5:1 and 4:1 dr, respectively). To our surprise, enyne 5k with a methyl substituent on the alkynyl position provided product $\mathbf{6 k}$ in high yield and diastereoselectivity $(81 \% ; 12: 1$ dr), which is in contrast with the results observed for $\mathbf{6 a}-\mathbf{6 b}$ (vide supra).

Demonstrated in Scheme 4 is the use of propargyl alcohol ()-S15 of 95:5 er $(90 \%$ ee $)$ for the synthesis of enyne (-)-5g (81\% yield; See ESI). Following our optimized PKR conditions, optically active cyclopentaoxasilinone (-)-6g was furnished in $92 \%$ yield with $98.5: 1.5$ er $(97 \%$ ee $)$, presumably as a result of adventitious enantiomeric enrichment during purification of enyne (-)-5g and PKR product (-)-6g. ${ }^{28}$ In all cases,
Scheme 3. Scope and Limitation of the Diastereoselective Siloxy-Tethered PKR ${ }^{a, b, c, d}$

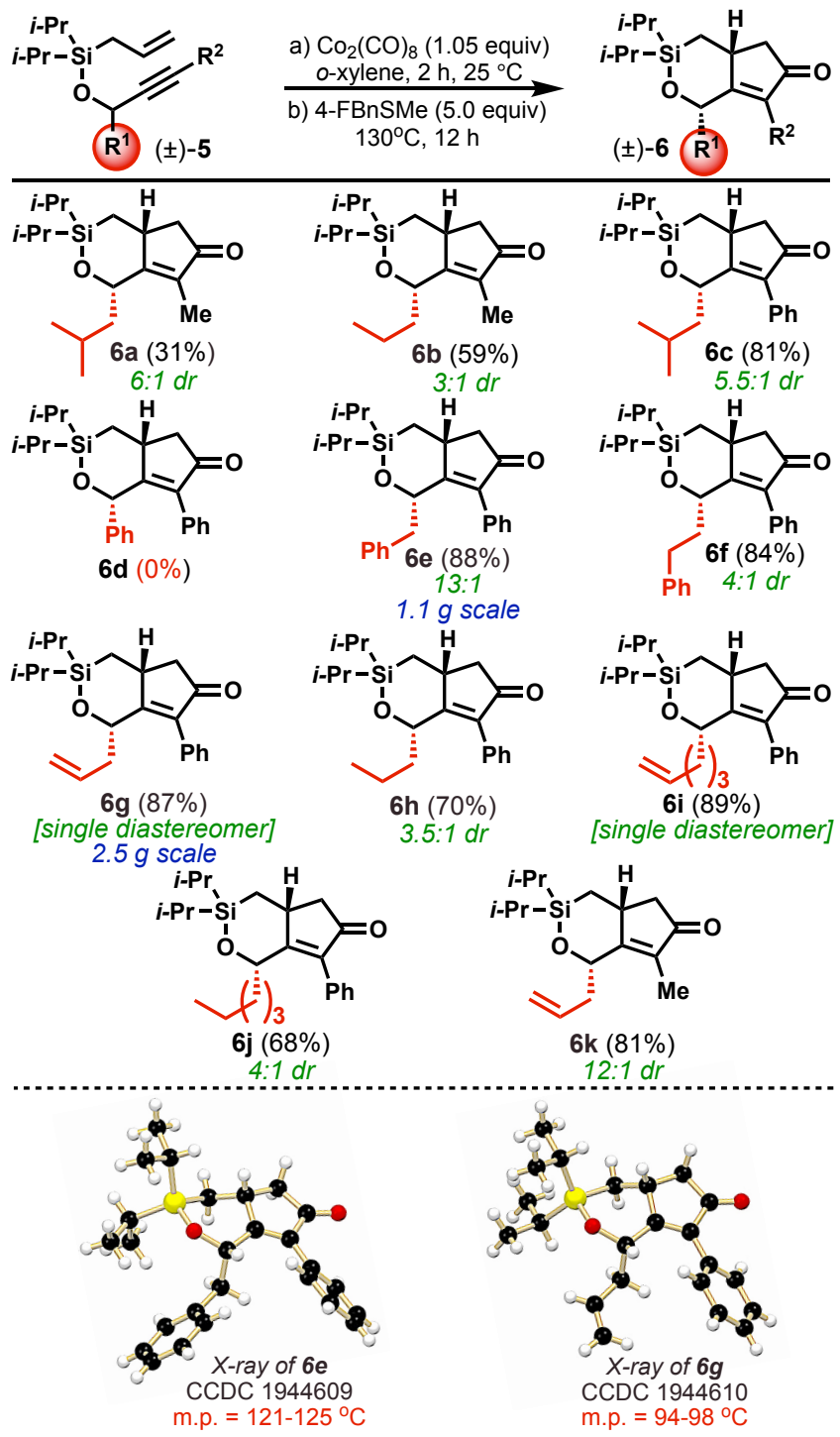

${ }^{a} 1.0$ equiv of $( \pm)-5 \mathbf{a}-\mathbf{k}, 0.33 \mathrm{mmol}$ scale $(0.10 \mathrm{M})$ unless otherwise noted. ${ }^{b}$ Purified/isolated yields represent an average of 3 experiments. ${ }^{c} 83 \%$ average recovery of 4 -FBnSMe. ${ }^{d}$ Diastereomer ratios were measured by ${ }^{1} \mathrm{H}$ NMR analysis of the unpurified material.

as illustrated in Schemes 3 and 4, the major PKR product possesses a syn relationship between the propargylic proton and the bridgehead proton of the newly formed stereocenter. The connectivity and selectivity of products $\mathbf{6 a}-\mathbf{k}$ was

Scheme 4. Synthesis of Enantio- and Diastereoenriched Cyclopentaoxasilinone (-)-6g ${ }^{a, b}$

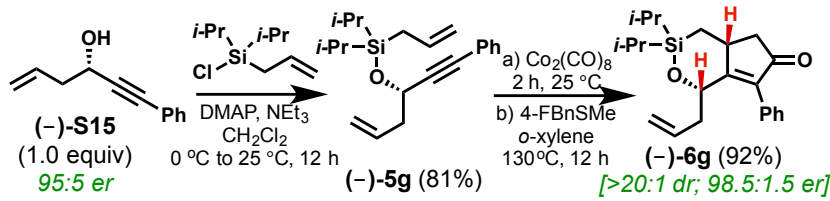

${ }^{a}$ The er value was determined by chiral HPLC analysis. ${ }^{b}$ Diastereomer ratios were measured by ${ }^{1} \mathrm{H}$ NMR analysis of the unpurified material. 
unambiguously established by single-crystal X-ray diffraction studies or by analysis of 2D NOESY experiments (See ESI). The improvement in diastereoselectivity observed for cyclopentaoxasilinones $\mathbf{6 e}, \mathbf{6 g}, \mathbf{6} \mathbf{i}$, and $\mathbf{6 k}$ is intriguing because it: (1) implicates the appended $\pi$-component as the key structural element for control of diastereoselectivity, (2) expands the synthetic utility of the products via a terminal olefin that provides a versatile handle for further synthetic manipulations, and (3) accommodates alkyl groups at the alkynyl position, whilst maintaining high yields and selectivity (cf. 6k and $\mathbf{6 a}-$ 6b).

As illustrated in Scheme 5, highly diastereoselective transformations of cyclopentaoxasilinone $\mathbf{6 e}$ and $\mathbf{6 g}$ rapidly

Scheme 5. Stereoselective Reactions Involving Cyclopentaoxasilinones (6e) and (6g) Showcasing Utility ${ }^{a}$

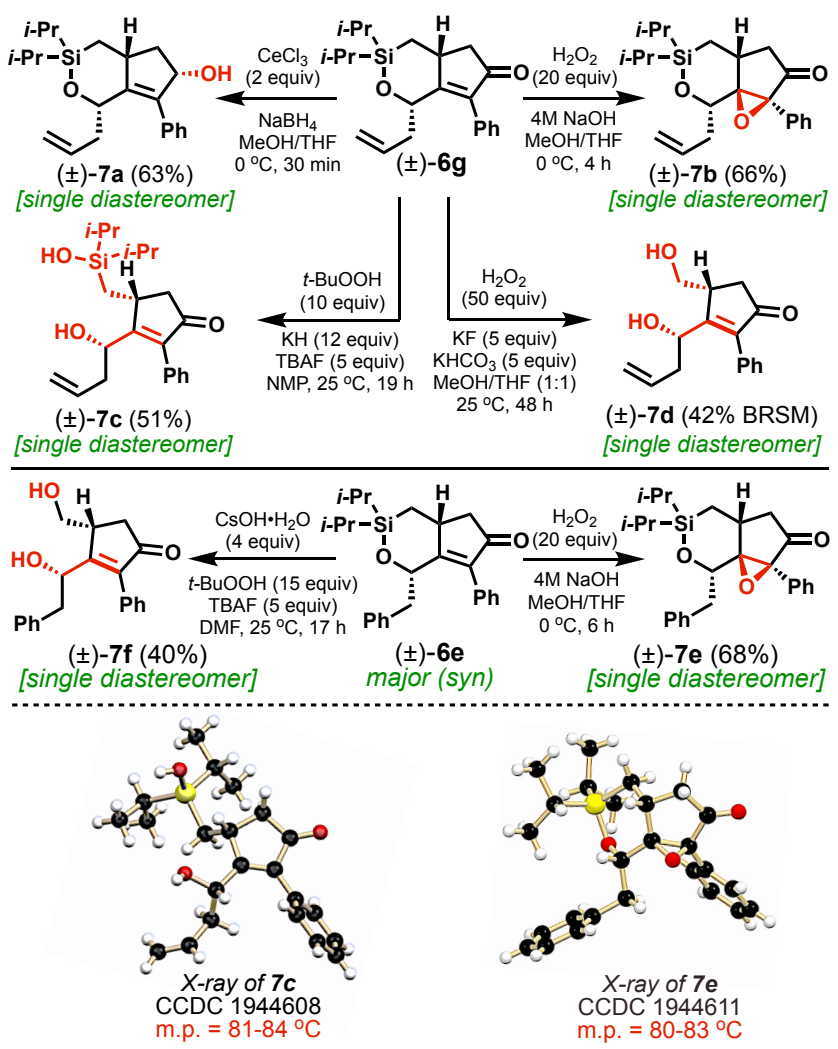

${ }^{a}$ Diastereomer ratios were measured by ${ }^{1} \mathrm{H}$ NMR analysis of the unpurified material.

introduce stereochemical complexity and structural diversity for the synthesis of useful synthetic building blocks. ${ }^{29}$ For example, subjecting $\mathbf{6 g}$ to cerium(III)-mediated Luche reduction $^{30}$ affords allylic alcohol $7 \mathbf{a}$ in $63 \%$ yield, which is poised for substrate-directed stereoselective transformations. ${ }^{20}$ Treatment of $\mathbf{6 e}$ and $\mathbf{6 g}$ with basic hydrogen peroxide provides $7 \mathbf{e}$ and $7 \mathbf{b}$ in $66 \%$ and $68 \%$ yields, respectively. Importantly these compounds were isolated as single diastereomers and are endowed with four contiguous stereocenters and an epoxide that can be opened in stereoselective fashion with a variety of nucleophiles. The stereochemical outcome is consistent with nucleophilic attack from the more sterically accessible convex face of the cyclopentaoxasilinone structure. Scission of the $\mathrm{Si}-$ $\mathrm{O}$ bond furnished silanol $7 \mathbf{c}$ in a $51 \%$ yield. Oxidative cleavage of the $\mathrm{Si}-\mathrm{C}$ bond $^{13}$ in $\mathbf{6 e}$ and $\mathbf{6 g}$ delivered the desired monocyclic cyclopentenones $7 \mathbf{d}$ in $42 \%$ (brsm; based on the re- covery of starting material $\mathbf{6 g}$ ), and $7 \mathbf{f}$ in $40 \%$ unoptimized yields. Notably, functionalized cyclopentenones $7 \mathbf{a}-\mathbf{7 f}$ were obtained as single diastereomers and confirmed via X-ray crystallographic analysis or 2D NOESY experiments (See ESI). The accessible nature of the precursors, modular onestep synthesis of the enynes, and the scalability of the siloxytethered PKR reaction bodes well for future synthetic applications.

In summary, we have developed a diastereoselective $\mathrm{Si}-\mathrm{O}$ tethered Pauson-Khand reaction that utilizes readily available propargyl alcohols for the synthesis of cyclopentaoxasilinones. The strategy presented provides stereoenriched/highly functionalized cyclopentenones that are otherwise not accessible via intermolecular PKRs. For example, oxidative cleavage of the $\mathrm{Si}-\mathrm{C}$ bond, enables access to stereoenriched $\beta$-alkylated cyclopentenones with pendant homo- and allylic diol moieties. A recoverable/reusable thioether, namely 4fluorobenzyl(methyl)sulfide (4-FBnSMe) was found to promote the reaction in improved yields. The utility of the PKR products is showcased in highly diastereoselective transformations to furnish cyclopentenones with multiple functional groups and stereocenters. Moreover, the cyclopentaoxasilinones are structural chemotypes that are of broad scientific interest. ${ }^{29} \mathrm{We}$ are currently working on understanding the selectivity and the fascinating role of the appended olefin/benzyl groups through a combination of computational and experimental studies. Further extensions of this method and applications in the total synthesis of natural products are currently ongoing in our Laboratory and will be reported in due course.

\section{ASSOCIATED CONTENT}

\section{Supporting Information}

The Supporting Information is available free of charge on the ACS Publications website.

Detailed experimental procedures, characterization data, and NMR spectra for all new compounds (PDF)

\section{Accession Codes}

CCDC 1944608 - 1944611 contain the supplementary crystal graphic data for this paper. These data can be obtained free of charge via www.ccdc.cam.ac.uk/data request/ cif, or by emailing data_request@ccdc.cam.ac.uk, or by contacting The Cambridge Crystallographic Data Centre, 12 Union Road, Cambridge CB2 1EZ, UK; fax: +44 1223336033 .

\section{AUTHOR INFORMATION}

\section{Corresponding Author}

*dionicio.s.martinez@drexel.edu

\section{Present Addresses}

§ Johnson Matthey, 436 Devon Park Drive, Wayne, PA 19087.

\section{Author Contributions}

${ }^{\perp}$ These authors contributed during initial stages of the project. Notes

The authors declare no competing financial interest.

\section{ACKNOWLEDGMENT}

Financial support for this project was provided by Drexel University (start-up funds). We thank Dr. Jacob R. Powell (NMR Specialist, Drexel), Dr. Timothy P. Wade, and Michelle L. Piotrowski 
(mass spectrometry, Drexel). We are grateful to Prof. Osvaldo Gutierrez (University of Maryland) for helpful discussions from the inception of the project. We are thankful to Prof. Simon Cocklin (Drexel) and Prof. Patrick J. Walsh (UPenn) for helpful suggestions during the writing of this manuscript, Dr. Chen $\mathrm{Wu}$ (Walsh Group, UPenn) for providing HPLC data, and former Martinez-Solorio research group members: Brendan G. Rackley, Adam W. McLean, Pasquale Carione (Genentech), and Karlo K. Sales. In memoriam: A Great Father and Teacher, Rosalio Martinez Aguayo; 9/4/1947 - 2/26/2019 (D.M.S.).

\section{REFERENCES}

(1) For selected reviews, see: (a) Simeonov, S. P.; Nunes, J. P. M.; Guerra, K.; Kurteva, V. B.; Afonso, C. A. M. Chem. Rev., 2016, 116, 5744 - 5893. (b) Aitken, D. J.; Eijsberg, H.; Frongia, A.; Ollivier, J.; Piras, P. P. Synthesis, 2014, 46, 00010 - 00024, DOI: 10.1055/s0033-1340414.

(2) Trost, B.M. Science, 1991, 254, 1471-1477.

(3) (a) R. Rios Torres, The Pauson Khand Reaction: Scope, Variations, and Applications, John Wiley \& Sons, Ltd., Chichester, U.K., 2012; For selected PKR reviews, see: (b) Ricker, J. D.; Geary, L. M. Topics in Catalysis, 2017, 60, 609 - 619. (c) Shi, L.; Yang, Z. Eur. J. Org. Chem., 2016, 2356 - 2368. (d) Lee, H.-W., Kwong, F.-Y. Eur. J. Org. Chem., 2010, $789-811$.

(4) For seminal PKR publications, see: (a) Khand, I. U.; Knox, G. R.; Pauson, P. L.; Watts, W. E.; Foreman, M. I. J. Chem. Soc., Perkin Trans. 1, 1973, 977 - 981 (b) Khand, I. U.; Knox, G. R.; Pauson, P. L.; Watts, W. E.; J. Chem. Soc. , Perkin Trans. 1, 1973, 975 - 977.

(5) For intermolecular PKR reviews, see: (a) Gibson, S. E.; Mainolfi, N. Angew. Chem. Int. Ed., 2005, 44, 3022 - 3037. (b) Laschat, S.; Becheanu, A.; Bell, T.; Baro, A. Synlett, 2005, 17, 2547 2570.

(6) For recent advances in the cobalt-catalyzed intermolecular PKR: (a) Kizirian, J.-C.; Aiguabella, N.; Pesquer, A.; Fustero, S.; Bello, P.; Verdaguer, X.; Riera, A. Org. Lett., 2010, 12, 5620 - 5623. (b) Revés, M.; Lledó, A.; Ji, Y.; Blasi, E.; Riera, A.; Verdaguer, X. Org. Lett., 2012, 14, 3534 - 3537. (c) Fager-Jokela, E.; Muuronen, M.; Khaizourane, H.; Vázquez-Romero, A.; Verdaguer, X.; Riera, A.; Helaja, J. J. Org. Chem., 2014, 79, 10999 - 11010. (d) Cabré, A.; Verdaguer, X.; Riera, A. Synthesis, 2017, 49, A - G, DOI: 10.1055/s0036-1588813.

(7) (a) Lledó, A.; Solà, J.; Verdaguer, X.; Riera, A.; Maestro, M. A. Adv. Synth. Catal.; 2007, 349, 2121 - 2128. (b) Sola, J.; Revés, M.; Riera, A.; Verdaguer, X. Angew. Chem. Int. Ed., 2007, 46, 5020 5023. (c) Revés, M.; Achard, T.; Solà, J.; Verdaguer, X.; Riera, A.; Helaja, J. J. Org. Chem., 2008, 73, 7080 - 7087. (d) Ferrer, C.; Riera, A.; Verdaguer, X. Organometallics, 2009, 28, 4571 - 4576. (e) Ji, Y.; Riera, A.; Verdaguer, X. Org. Lett., 2009, 11, 4346 - 4349. (f) Orgué, S.; León, T.; Riera, A.; Verdaguer, X. Org. Lett., 2015, 17, 250 - 253.

(8) (a) Rodríguez Rivero, M.; De la Rosa, J. C.; Carretero, J. C. J. Am. Chem. Soc. 2003, 125, 14992 - 14993. (b) Rodríguez Rivero, M.; Alonso, I.; Carretero, J. C. Chem. Eur. J., 2004, 10, 5443 - 5459.

(9) (a) Miller, K. A.; Martin, S. F. Org. Lett., 2007, 9, 1113 - 1116. (b) Liu, D. D.; Sun, T. W.; Wang, K. Y.; Lu, Y.; Zhang, S. L.; Li, Y. H.; Jiang, Y. L.; Chen, J. H.; Yang, Z. J. Am. Chem. Soc., 2017, 139, 5732 - 5735. (c) Zhao, N.; Yin, S.; Xie, S.; Yan, H.; Ren, P.; Chen, G.; Chen, F.; Xu, J. Angew. Chem. Int. Ed., 2018, 57, 3386 - 3390. (d) Zhang, W.; Ding, M.; Li, J.; Guo, Z.; Lu, M.; Chen, Y.; Liu, L.; Shen, Y. H.; Li, A. J. Am. Chem. Soc., 2018, 140, 4227 - 4231

(10) For rare examples of intermolecular PKRs in total synthesis of natural products, see: (a) Iqbal, M.; Evans, P.; Lledó, A.; Verdaguer, X.; Pericàs, M. A.; Riera, A.; Loeffler, C.; Sinha, A. K.; Mueller, M. J. Chembiochem 2005, 6, 276 - 280. (b) Jiang, B.; Li, M.-M.; Xing, P.; Huang, Z.-G. Org. Lett., 2013, 15, 871 - 873. (c) Rodriguez, R. A.; Barrios Steed, D.; Kawamata, Y.; Su, S.; Smith, P. A.; Steed, T. C.; Romesberg, F. E.; Baran, P. S. J. Am. Chem. Soc., 2014, 136, $15403-15413$.

(11) F. Diederich and P. J. Stang, Templated Organic Synthesis, Wiley-VCH, Verlag GmbH, D-69469 Weinheim, Federal Republic of Germany, 2000.
(12) For seminal research employing silicon tethers, see: (a) Nishiyama, H.; Kitajima, T.; Matsumoto, M.; Itoh, K. J. Org. Chem., 1984, 49, 2298 - 2300. (b) Stork, G.; Kahn, M. J. Am. Chem. Soc., 1985, 107, 500 - 501; For a review, see: (a) Bracegirdle, S.; Anderson, E. A. Chem. Soc. Rev., 2010, 39, $4114-4129$.

(13) For seminal Tamao-Fleming reports, see: (a) Tamao, K.; Akita, M.; Kumada, M. J. Organomet. Chem. 1983, 254, 13 - 22. (b) Tamao, K.; Ishida, N.; Tanaka, T.; Kumada, M. Organometallics 1983, 2, 1694 - 1696. (c) Tamao, K.; Kakui, T.; Akita, M.; Iwahara, T.; Kanatani, R.; Yoshida, J.; Kumada, M. Tetrahedron 1983, 39, 983 - 990. (d) Fleming, I.; Henning, R.; Plaut, H. J. Chem. Soc., Chem. Commun. 1984, 29 - 31. (e) Tamao, K.; Ishida, N. J. Organomet. Chem. 1984, 269, C37 - C39. (f) Fleming, I.; Sanderson, P. E. J. Tetrahedron Lett. 1987, 28, 4229-4232. For a review on stereoselective silicon transformations, see: (g) Fleming, I.; Barbero, A.; Walter, D. Chem. Rev. 1997, 97, 2063 - 2192.

(14) (a) Kagoshima, H.; Hayashi, M.; Hashimoto, Y.; Saigo, K. Organometallics, 1996, 15, 5439 - 5441. (b) Reichwein, J. F.; Iacono, S. T.; Pagenkopf, B. L. Tetrahedron, 2002, 58, 3813 - 3822. (c) Reichwein, J. F.; Iacono, S. T.; Patel, M. C.; Pagenkopf, B. L.; Tetrahedron Lett., 2002, 43, 3739 - 3741. (d) Brummond, K. M.; Sill, P. C.; Rickards, B.; Geib, S, J. Tetrahedron Lett., 2002, 43, 3735 - 3738. (e) Ishaq, S.; Porter, M. J. Synthetic Communications, 2006, 36, 547 557. (f) Dobbs, A. P.; Miller, I. J.; Martinovic, S. Beilstein J. Org. Chem., 2007, 3, No. 21.

(15) Sugihara, T.; Yamada, M.; Yamaguchi, M.; Nishizawa, M. Synlett 1999, $771-773$.

(16) For rare examples of PKRs employing enantioenriched propargyl alcohols, see: (a) Cai, H.; Nie, J.; Zheng, Y.; Ma, J.-A. J. Org. Chem., 2014, 79, 5484 - 5493. (b) Chen, W.; Tay, J.-H.; Ying, J.; Yu, X.-Q.; Pu, L. J. Org. Chem., 2013, 78, 2256 - 2265. (c) Turlington, M.; Yue, Y.; Yu, X.-Q.; Pu, L. J. Org. Chem., 2010, 75, 6941-6952. (d) Sezer, S.; Özdemirhan, D.; Sahin, E.; Tanyeli, C. Tetrahedron Asymmetry, 2006, 17, 2981 - 2986. (e) Stumpf, A.; Hwang, S. H.; Kim, J. S.; Jeong, N. Bull. Korean Chem. Soc., 2004, 25, 1621 1622.

(17) Asymmetric transfer hydrogenation: a) Matsumura, K.; Hashiguchi, S.; Ikariya, T.; Noyori, R. J. Am. Chem. Soc. 1997, 119, 8738 - 8739; Asymmetric hydrogenation: b) Noyori, R.; Okuma, T.; Tsutsumi, K.; Utsumi, N.; Murata, K. US pat. US20070225528, 2007.; Jap. pat., JP2010285443,2010; c) Arai, N.; Satoh, H.; Utsumi, N.; Murata, K.; Tsutsumi, K.; Ohkuma, T. Org.Lett. 2013, 15, 3030 3033.

(18) For a review on asymmetric allylations, see: Yus, M.; Gonzalez-Gomez, G. C.; Foubelo, F. Chem. Rev. 2013, 113, 5595 - 5698.

(19) Asymmetric alkynylations: (a) Frantz, D. E.; Fässler, R.; Tomooka, C. S.; Carreira, E. M. Acc. Chem. Res. 2000, 33, 373 - 381. (b) $\mathrm{Pu}, \mathrm{L}$. Tetrahedron 2003, 59, 9873 - 9886.

(20) For a discussion on allylic alcohol asymmetric directed reactions, see: (a) Bhadra, S.; Yamamoto, H. Chem. Rev. 2018, 118, 3391 - 3446. For a recent mini-review on allylic alcohols, see: (b) Zhang, J.; Liao, J.; Wei, Y.-F.; Cheng, G.; Luo, R. Mini-Reviews in Organic Chemistry, 2018, 15, 476-487. For a review on synthesis and applications of allylic alcohols, see: (c) Lumbroso, A.; Cooke, M. L.; Breit, B. Angew. Chem. Int. Ed. 2013, 52, 1890 - 1932.

(21) Shambayati, S.; Crewe, W. E.; Schreiber, S. L. Tetrahedron Letters, 1990, 31, 5289 - 5292.

(22) Jeong, N.; Chung, Y. K.; Lee, B. Y.; Lee, S. H.; Yoo, S.-E. Synlett, 1991, 3, $204-206$.

(23) Sugihara, T.; Yamada, M.; Ban, H.; Yamaguchi, M.; Kaneko, C. Angew. Chem., Int. Ed. Engl. 1997, 36, $2801-2804$.

(24) Magnus, P.; Principe, L. M.; Tetrahedron Letters, 1985, 26 , $4851-4854$

(25) (a) Krafft, M. E.; Scott, I. L.; Romero, R. H.; Feibelmann, S.; Van Pelt, C. E. J. Am. Chem. Soc. 1993, 115, 7199 - 7207. (b) Yamanaka, M.; Nakamura, E. J. Am. Chem. Soc. 2001, 123, $1703-1708$. (c) Del Valle, C. P.; Milet, A.; Gimbert, Y.; Greene, A. E. Angew. Chem. Int. Ed. 2005, 44, 5717 - 5719.

(26) For a review on the Thorpe-Ingold effect directly relating to organosilicon compounds, see: Luh, T.-Y.; Hua, Z. Dalton Trans. 2010, 39, 9185 - 9192 . 
(27) Phenyl substituted propargyl alcohols or enynes such as $\mathbf{5 d}$ undergo $\mathrm{C}-\mathrm{O}$ heterolytic cleavage due to the facile formation of a cobalt-stabilized benzylic cation. For a relevant review of the Nicholas reaction, see: (a) Nicholas, K. M. Acc. Chem. Res. 1987, 20, 207 214.

(28) Martens J.; Bhushan, R. Helv. Chim. Acta, 2014, 97, $161-$
(29) For reviews on organosilicon compounds for medicinal chemistry applications, see: (a) Ramesh R.; Reddy, D. S. J. Med. Chem., 2018, 61, 3779 - 3798. (b) Franz A. K.; Wilson, S. O. J. Med. Chem., 2013, $56,388-405$.

(30) Luche, J.-L. J. Am. Chem. Soc. 1978, 100, 2226 - 2227. 187. 


\section{Supporting Information}

\section{Access to Highly Functionalized Cyclopentenones via Diastereoselective Pauson Khand Reaction of Siloxy-Tethered 1,7-Enynes}

Austin G. Gallagher, ${ }^{\dagger}$ Huan Tian,${ }^{\dagger}$ Osmar A. Torres-Herrera,${ }^{\dagger}$ Shuai Yin, ${ }^{\dagger}$ Anxin Xie, ${ }^{\dagger}$ Daniel M. Lange,${ }^{\dagger \S}$ Jerica K. Wilson, ${ }^{\dagger 1}$ Louis G. Mueller, ${ }^{\dagger \perp}$ Michael R. Gau, ${ }^{\ddagger}$ Patrick J. Carroll, ${ }^{\ddagger}$ and Dionicio Martinez-Solorio*'

${ }^{\dagger}$ Department of Chemistry, Drexel University, 32 S. 32nd Street, Philadelphia, Pennsylvania 19104, United States

* Department of Chemistry, University of Pennsylvania, 231 South 34th Street, Philadelphia, Pennsylvania 19104, United States

Corresponding Author Email: dionicio.s.martinez@drexel.edu

\section{Table of Contents}

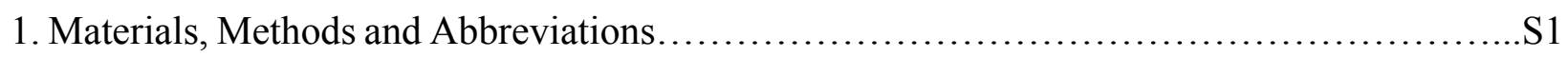

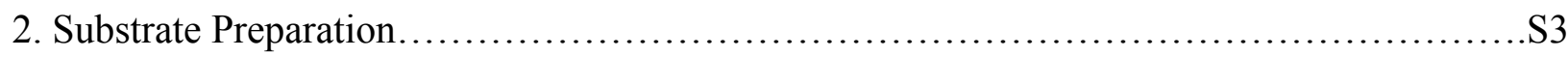

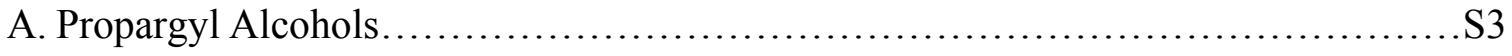

B. General Procedure for the Syntheses of 1,7-Siloxy-Tethered Enynes................S4

3. Pauson-Khand Reaction (PKR) of 1,7-Siloxy-Tethered Enynes ........................ 15

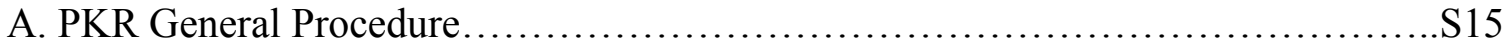

B. Relative Stereochemistry Determination................................... 16

C. Enantioenriched Substrates...........................................S29

D. Functionalization of Cyclopentaoxasilinones................................. 32

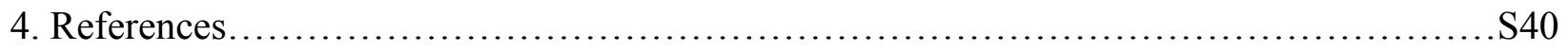

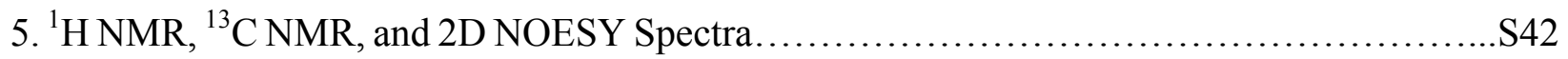




\section{Materials and Methods}

Unless otherwise stated, all reactions were performed using syringe-septum cap techniques under an inert atmosphere of $\mathrm{N}_{2}$. All reagent grade solvents and chemicals were purchased from commercial vendors and used without further purification, unless otherwise stated. All reactions were magnetically stirred in oven- or vacuum flame-dried glassware with commercially available anhydrous solvents; tetrahydrofuran (THF), diethyl ether $\left(\mathrm{Et}_{2} \mathrm{O}\right)$, methylene chloride $\left(\mathrm{CH}_{2} \mathrm{Cl}_{2}\right)$, toluene (PhMe), and $o$-xylenes, unless otherwise noted. Anhydrous N-methylpyrrolidinone (NMP) was purchased from Aldrich and stored under $\mathrm{N}_{2}$. Unless otherwise stated, chemicals and reagents were used as received. All reactions were monitored by thin-layer chromatography (TLC) using $250 \mu \mathrm{m}$ precoated silica gel plates and were visualized by staining with $\mathrm{KMnO}_{4}\left(1.5 \mathrm{~g}\right.$ of $\mathrm{KMnO}_{4}$, $10 \mathrm{~g}$ of $\mathrm{K}_{2} \mathrm{CO}_{3}$ and $2.5 \mathrm{~mL}$ of $5 \%$ aq. $\mathrm{NaOH}$ in $150 \mathrm{~mL} \mathrm{H} \mathrm{H}_{2} \mathrm{O}$ ), or CAM (4.8 $\mathrm{g}$ of $\left(\mathrm{NH}_{4}\right)_{6} \mathrm{Mo}_{7} \mathrm{O}_{24} \cdot 4 \mathrm{H}_{2} \mathrm{O}$ and $0.2 \mathrm{~g}$ of $\mathrm{Ce}\left(\mathrm{SO}_{4}\right)_{2}$ in $100 \mathrm{~mL}$ of a $3.5 \mathrm{~N} \mathrm{H}_{2} \mathrm{SO}_{4}$ solution) stain. . Flash column chromatography was performed as described by Still et al. ${ }^{1}$ using silica gel (230-400 mesh, Silicycle). Purified compounds were dried on a high vacuum line ( $2-6$ torr) to remove trace solvent. Yields refer to chromatographically and spectroscopically pure compounds, unless otherwise stated. All melting points were obtained on a Thomas-Hoover apparatus and are uncorrected. Optical rotations were measured on a Jasco P-2000 polarimeter using a $100 \mathrm{~mm}$ pathlength cell at $589 \mathrm{~nm}$ at the University of Pennsylvania. ${ }^{1} \mathrm{H}$ and ${ }^{13} \mathrm{C}$ NMR spectra were recorded on a Varian $400 \mathrm{MR}$ (at $400 \mathrm{MHz}$ and $101 \mathrm{MHz}$, respectively), or a Varian Inova 500 (at $500 \mathrm{MHz}$ and $126 \mathrm{MHz}$, respectively). NMR data is reported relative to internal $\mathrm{CDCl}_{3}\left({ }^{1} \mathrm{H}, \delta=7.26\right), \mathrm{CDCl}_{3}$ $\left({ }^{13} \mathrm{C}, \delta=77.16\right)$. Data for ${ }^{1} \mathrm{H}$ NMR spectra are reported as follows: chemical shift $(\delta \mathrm{ppm})$ (multiplicity, coupling constant (Hz), integration). Multiplicity and qualifier abbreviations are as follows: $(\mathrm{s}=$ singlet, $\mathrm{d}=$ doublet, $\mathrm{t}=$ triplet, $\mathrm{q}=$ quartet, $\mathrm{qn}=$ quintet, $\mathrm{dd}=$ doublet of doublets, $\mathrm{ddd}=$ doublet of doublet of doublets, $\mathrm{ddt}=$ doublet of doublet of triplets, $\mathrm{dt}=$ doublet of triplets, thept $=$ triplet of heptets, $\mathrm{m}=$ multiplet, $\mathrm{b}=$ broad). ${ }^{13} \mathrm{C}$-NMR spectra are tabulated by observed peak. IR spectra were recorded on a PerkinElmer 100 FT-IR spectrometer and are reported in frequency of absorption (cm-1). Analytical chiral SFC was performed with a Mettler SFC supercritical $\mathrm{CO}_{2}$ analytical chromatography system $\left(\mathrm{CO}_{2}=1450\right.$ psi, column temperature $=40$ ${ }^{\circ} \mathrm{C}$ ) with Chiralcel AD-H, OD-H, columns (4.6 mm x $\left.25 \mathrm{~cm}\right)$. High-resolution mass spectra (HRMS) were acquired at the Drexel Mass Spectral Facility on a Thermo-Electron LTQ-FT 7T 
Fourier transform ion cyclotron resonance (FT-ICR) Spectrometer with an atmospheric pressure chemical ionization (APCI) source with direct infusion run in positive ion mode at $5 \mathrm{kV}$.

\section{X-Ray Structure Determination (University of Pennsylvania)}

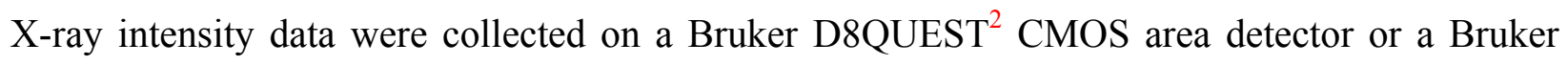
$\mathrm{APEXII}^{3}$ CCD area detector employing graphite-monochromated Mo-K $\alpha$ radiation $(\lambda=0.71073 \AA)$ at a temperature of $100 \mathrm{~K}$. Preliminary indexing was performed from a series of twenty-four $0.5^{\circ}$ rotation frames with exposures of 10 seconds. Rotation frames were integrated using SAINT, ${ }^{4}$ producing a listing of unaveraged $\mathrm{F}^{2}$ and $\sigma\left(\mathrm{F}^{2}\right)$ values. The intensity data were corrected for Lorentz and polarization effects and for absorption using SADABS. ${ }^{5}$ The structure was solved by direct methods - ShelXT ${ }^{6}$ or ShelXS-97. ${ }^{7}$ Refinement was by full-matrix least squares based on $\mathrm{F}^{2}$ using SHELXL-2018. ${ }^{8}$ All reflections were used during refinement. Non-hydrogen atoms were refined anisotropically and hydrogen atoms were refined using a riding model. Crystallographic data for compounds can be obtained free of charge from The Cambridge Crystallographic Data Centre (CCDC) via www.ccdc.cam.ac.uk/data_request/cif under CCDC deposition numbers 1944608-1944611. Graphical representation of structures with 50\% probability thermal ellipsoids was generated using Mercury visualization software. ${ }^{9}$

\section{Abbreviations:}

4-OMeBnSMe - 4-Methoxybenzyl methyl sulfide; 4-FBnSMe - (4fluorobenzyl)(methyl)sulfide; BnSMe - Benzyl methyl sulfide; n-BuSMe - nButyl methyl sulfide; CAM - cerium ammonium molybdate; $\mathbf{C y N H}$ - Cyclohexylamine; DCM dichloromethane; DMAP - 4-dimethylaminopyridine; DMF - dimethylformamide; dr diastereomeric ratio; er - enantiomeric ratio; $\mathbf{E t}_{2} \mathbf{O}$ - diethyl ether; EtOAc - ethyl acetate; FTIR - Fourier transform infrared; FurfurylSMe - 2-((methylthio)methyl)furan; NMO - NMethylmorpholine N-Oxide; NMP - N-methylpyrrolidinone; NMR - nuclear magnetic resonance; PhMe - toluene; PhSMe - methyl(phenyl)sulfide; SFC - supercritical fluid chromatography; THF - tetrahydrofuran; TLC - thin layer chromatography; TMANO Trimethylamine N-Oxide $\mathbf{U V}$ - ultraviolet 


\section{Substrate Preparation}

\section{A. Propargyl Alcohols}

The synthesis of the propargyl alcohols S1 - (-)-S19, ${ }^{10-29}$ allyldiisopropylchlorosilane S20 ${ }^{30}$ and (4-fluorobenzyl)(methyl)sulfide (4-FBnSMe) $\mathbf{S 2 1}^{31}$ were prepared according to previously reported procedures. All the NMR spectra and analytical data of known compounds were in accordance with the reported references. Please see these references for characterization and supporting information.
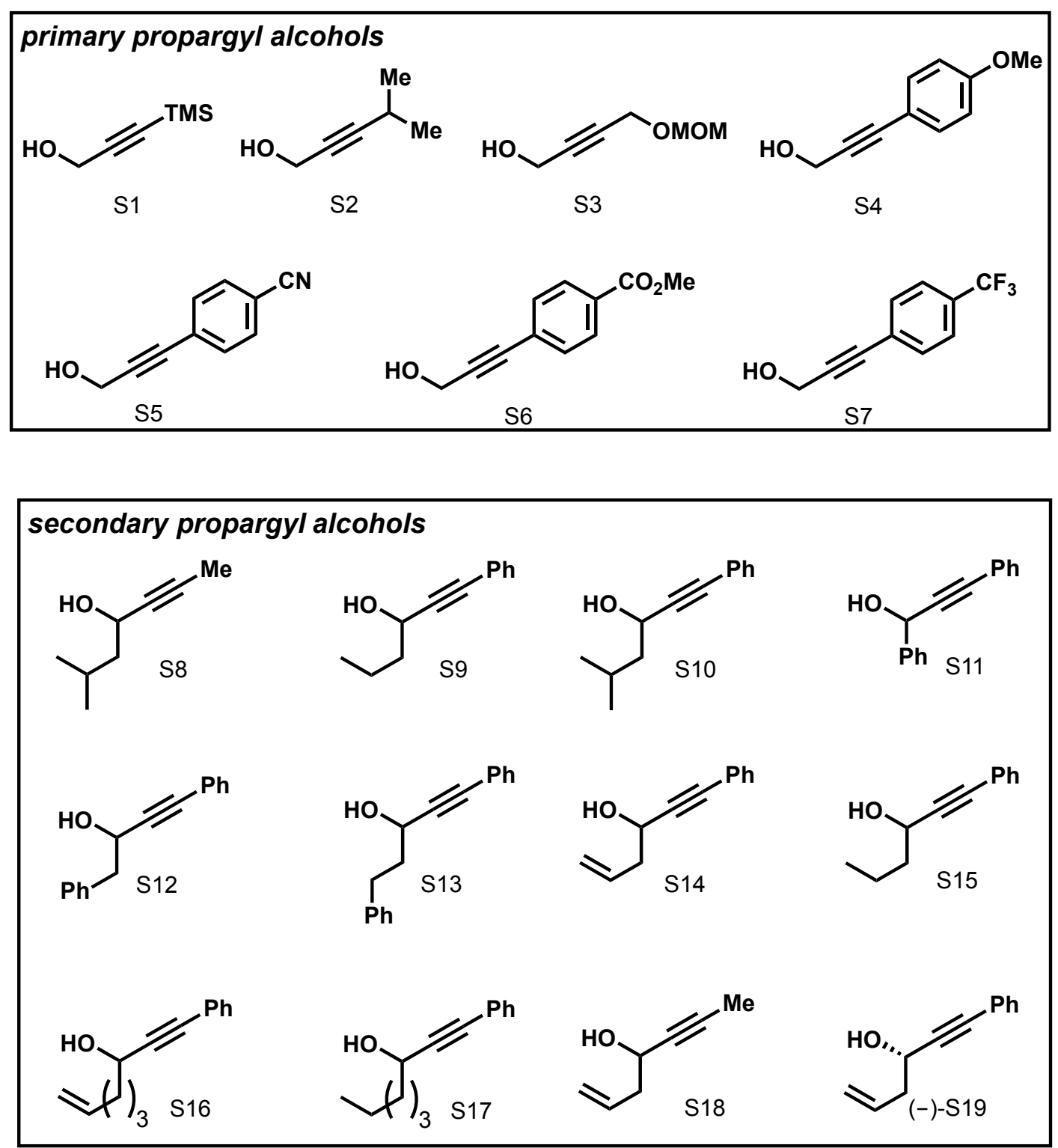


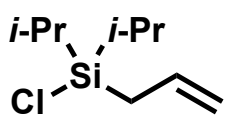

S20

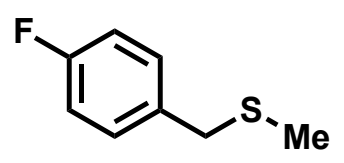

S21

Propargyl alcohols S22 - S24 purchased commercial sources and used as is without further purification.

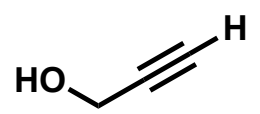

S22

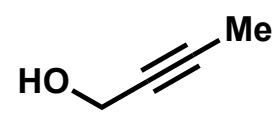

S23

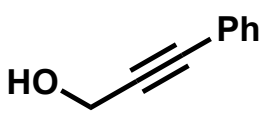

S24

B. 1,7-Siloxy-Tethered Enynes

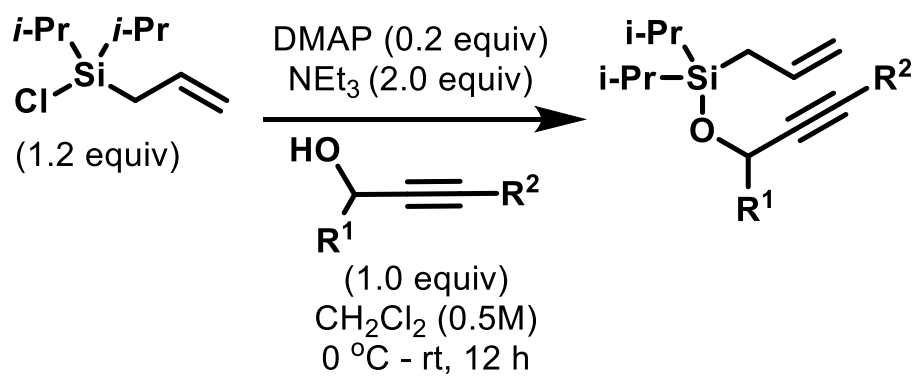

\section{General Procedure 1: 1,7-Siloxy-Tethered Enyne Synthesis}

A flame dried round-bottom flask equipped with a magnetic stir-bar was sequentially charged with dry $\mathrm{CH}_{2} \mathrm{Cl}_{2}$ (0.5 M with respect to the propargyl alcohol), allyldiisopropylchlorosilane $\mathbf{S 2 0}{ }^{29}$ (1.2 equiv), DMAP (0.2 equiv), and $\mathrm{NEt}_{3}$ (2.0 equiv) under an inert atmosphere $\left(\mathrm{N}_{2}\right)$. The reaction mixture was cooled to $0{ }^{\circ} \mathrm{C}$. In a separate flame-dried pear-shaped flask, the propargyl alcohol (S1 - $\mathbf{S 1 9}$ and $\mathbf{S 2 2}-\mathbf{S 2 4}$; 1.0 equiv) was dissolved in $\mathrm{CH}_{2} \mathrm{Cl}_{2}(1.0 \mathrm{M})$ and added dropwise to the $0{ }^{\circ} \mathrm{C}$ reaction mixture via syringe. This was followed by rinsing the pear-shaped flask with an additional portion of $\mathrm{CH}_{2} \mathrm{Cl}_{2}(0.3 \mathrm{~mL})$. The cooling bath was removed, and the reaction mixture allowed to stir at room temperature $\left(25^{\circ} \mathrm{C}\right)$ for $18 \mathrm{~h}$. Upon consumption of the propargyl alcohol, as noted by TLC analysis, the reaction mixture was quenched with sat. aq. $\mathrm{NH}_{4} \mathrm{Cl}$. The aqueous phase was extracted with $\mathrm{CH}_{2} \mathrm{Cl}_{2}(3 \times 20 \mathrm{~mL})$, and the combined organic phases were washed with brine, dried with $\mathrm{MgSO}_{4}$, filtered, and concentrated under reduced pressure. Flash chromatography (hexanes - 0.25\% $\mathrm{Et}_{2} \mathrm{O} /$ hexanes) yielded enynes $\mathbf{1 a - j}$ and $\mathbf{5 a - k}$ as colorless oils. 


\section{Allyl(but-2-yn-1-yloxy)diisopropylsilane (1a)}

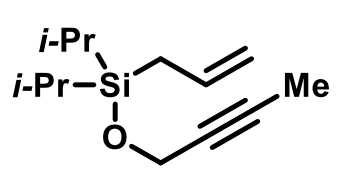

77\% yield (2.47 g, $11.0 \mathrm{mmol})$. Prepared from but-2-yn-1-ol S23 (1.00 g, $14.3 \mathrm{mmol}, 1.00$ equiv) and allylchlorodiisopropylsilane $\mathbf{S 2 0}(3.18 \mathrm{~mL}$, $3.27 \mathrm{~g}, 17.2 \mathrm{mmol}, 1.20$ equiv).

$\mathbf{R}_{f}: 0.8$ (silica, 1\% EtOAc/hexanes).

${ }^{1}$ H NMR (400 MHz, $\left.\mathbf{C D C l}_{3}\right)$ : $\delta 5.90(\mathrm{ddt}, J=17.0,10.1,8.1 \mathrm{~Hz}, 1 \mathrm{H}), 4.97$ (ddt, $J=17.0,2.2,1.3$ $\mathrm{Hz}, 1 \mathrm{H}), 4.87$ (ddt, $J=10.1,2.2,1.1 \mathrm{~Hz}, 1 \mathrm{H}), 4.33$ (q, $J=2.4 \mathrm{~Hz}, 2 \mathrm{H}), 1.84$ (t, $J=2.4 \mathrm{~Hz}, 3 \mathrm{H})$, $1.75(\mathrm{dt}, J=8.1,1.3 \mathrm{~Hz}, 2 \mathrm{H}), 1.10-0.99(\mathrm{~m}, 14 \mathrm{H})$.

${ }^{13}$ C NMR (101 MHz, $\left.\mathbf{C D C l}_{3}\right): \delta 134.56,113.97,81.08,77.83,52.35,18.95,17.58,17.57,12.64$, 3.75 .

IR (neat, $\mathbf{c m}^{-1}$ ): 2944, 2867, 1630, 1464, 1371, 1263, 1145, 1064, 994, 882, 774.

HRMS $\left(\mathbf{C I}^{+}, \boldsymbol{m} / \boldsymbol{z}\right)$ : calculated for $\mathrm{C}_{13} \mathrm{H}_{23} \mathrm{OSi}[\mathrm{M}-\mathrm{H}]$ 233.15127, found 223.15146.

\section{Allyldiisopropyl(prop-2-yn-1-yloxy)silane (1b)}

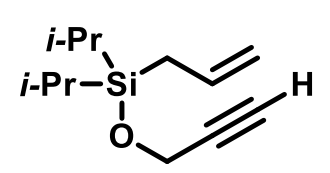

76\% yield (1.43 g, $6.78 \mathrm{mmol})$. Prepared from prop-2-yn-1-ol S22 (500 mg, $8.92 \mathrm{mmol}, 1.00$ equiv) and allylchlorodiisopropylsilane $\mathbf{S 2 0}$ (1.99 mL, 2.04 g, 10.7 mmol, 1.20 equiv).

$\mathbf{R}_{f}: 0.8$ (silica, 1\% EtOAc/hexanes).

${ }^{1}$ H NMR (400 MHz, $\mathbf{C D C l}_{3}$ ): $\delta 5.89$ (ddt, $\left.J=16.9,10.0,1.3 \mathrm{~Hz}, 1 \mathrm{H}\right), 4.99$ (ddt, $J=16.9,2.2,1.5$ $\mathrm{Hz}, 1 \mathrm{H}), 4.89$ (ddt, $J=10.0,2.2,1.1 \mathrm{~Hz}, 1 \mathrm{H}), 4.38$ (dd, $J=2.4,0.6 \mathrm{~Hz}, 2 \mathrm{H}), 2.40$ (td, $J=2.4,0.6$ $\mathrm{Hz}, 1 \mathrm{H}), 1.77(\mathrm{dt}, J=8.1,1.3 \mathrm{~Hz}, 2 \mathrm{H}), 1.10-1.03(\mathrm{~m}, 14 \mathrm{H})$.

${ }^{13}$ C NMR (101 MHz, $\left.\mathbf{C D C l}_{3}\right): \delta 134.33,114.18,82.40,73.04,51.95,18.86,17.56,17.53,12.61$. IR (neat, $\mathbf{c m}^{-1}$ ): 2944, 2867, 1630, 1464, 1370, 1264, 1160, 1094, 994, 882, 749.

HRMS $\left(\mathbf{C I}^{+}, \boldsymbol{m} / \boldsymbol{z}\right)$ : calculated for $\mathrm{C}_{12} \mathrm{H}_{21} \mathrm{OSi}[\mathrm{M}-\mathrm{H}] 209.13562$, found 209.13573.

\section{Allyldiisopropyl((3-(trimethylsilyl)prop-2-yn-1-yl)oxy)silane (1c)}

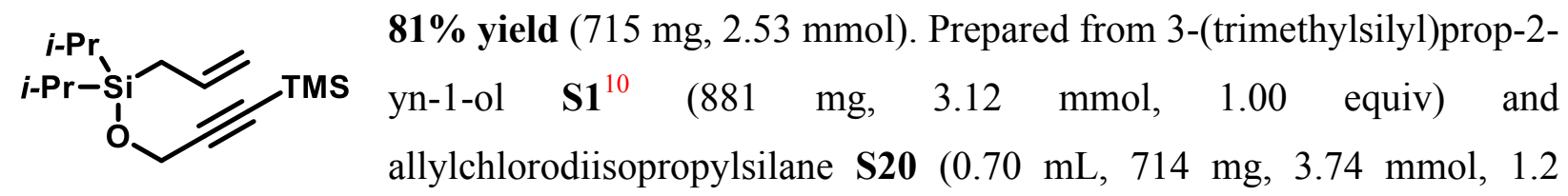
equiv).

$\mathbf{R}_{f}: 0.8$ (silica, 1\% EtOAc/hexanes). 
${ }^{1}$ H NMR (400 MHz, $\left.\mathbf{C D C l}_{3}\right): \delta 5.89$ (ddt, $\left.J=17.0,10.0,8.1 \mathrm{~Hz}, 1 \mathrm{H}\right), 4.97(\mathrm{dt}, J=17.0,2.2,1.4$ $\mathrm{Hz}, 1 \mathrm{H}), 4.86$ (ddt, $J=10.0,2.2,1.1 \mathrm{~Hz}, 1 \mathrm{H}), 4.36$ (s, 2H), 1.76 (dd, $J=8.1,1.3 \mathrm{~Hz}, 2 \mathrm{H}), 1.09$ $1.02(\mathrm{~m}, 14 \mathrm{H}), 0.16$ (s, 9H).

${ }^{13}$ C NMR (101 MHz, $\left.\mathbf{C D C l}_{3}\right): \delta 134.46,114.01,104.52,89.82,52.66,18.97,17.57,17.54,12.70$, -0.12 .

IR (neat, $\mathbf{c m}^{-1}$ ): 2945, 2867, 2177, 1630, 1463, 1364, 1250, 1094, 1001, 882, 840, 758.

HRMS $\left(\mathbf{C I}^{+}, \boldsymbol{m} / \boldsymbol{z}\right)$ : calculated for $\mathrm{C}_{15} \mathrm{H}_{30} \mathrm{OSi}_{2}[\mathrm{M}+]$ 282.18297, found 2822.18313.

Allyldiisopropyl((4-methylpent-2-yn-1-yl)oxy)silane (1d)

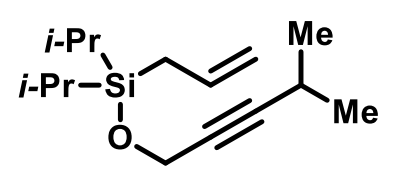

74\% yield (952 mg, 3.77 mmol). Prepared from 4-methylpent-2-yn-1-ol S2 $^{11}$ (500 mg, $5.09 \mathrm{mmol}, 1.00$ equiv) and allylchlorodiisopropylsilane S20 (1.14 mL, $1.17 \mathrm{~g}, 6.11 \mathrm{mmol}, 1.20$ equiv).

$\mathbf{R}_{f}: 0.8$ (silica, $1 \%$ EtOAc/hexanes).

${ }^{1}$ H NMR (400 MHz, $\left.\mathbf{C D C l}_{3}\right): \delta 5.90$ (ddt, $\left.J=17.0,10.1,8.1 \mathrm{~Hz}, 1 \mathrm{H}\right), 5.01-4.92(\mathrm{~m}, 1 \mathrm{H}), 4.90$ $-4.84(\mathrm{~m}, 1 \mathrm{H}), 4.36(\mathrm{dd}, J=6.8,2.0 \mathrm{~Hz}, 3 \mathrm{H}), 2.57$ (thept, $J=6.8,2.0 \mathrm{~Hz}, 1 \mathrm{H}), 1.76(\mathrm{dt}, J=8.0$, $1.3 \mathrm{~Hz}, 3 \mathrm{H}), 1.16(\mathrm{dd}, J=6.8,0.5 \mathrm{~Hz}, 6 \mathrm{H}), 1.06(\mathrm{qd}, J=2.5,0.9 \mathrm{~Hz}, 14 \mathrm{H})$.

${ }^{13}$ C NMR (101 MHz, $\left.\mathbf{C D C l}_{3}\right): \delta 134.67,113.90,91.02,77.97,52.37,22.99,20.70,19.03,17.61$, $17.59,12.70$.

IR (neat, $\mathbf{c m}^{-1}$ ): 2967, 2942, 2866, 2246, 1630, 1463, 1419, 1384, 1396, 1319, 1262, 1185, 1159, 1127, 1088, 1035, 994, 932, 882, 775, 749, 657.

HRMS (CI $\left.\mathbf{C I}^{+} \boldsymbol{m} / \boldsymbol{z}\right)$ : calculated for $\mathrm{C}_{15} \mathrm{H}_{29} \mathrm{OSi}[\mathrm{M}+\mathrm{H}]^{+}$253.19822, found 253.19814 .

10,10-Diisopropyl-2,4,9-trioxa-10-silatridec-12-en-6-yne (1e)

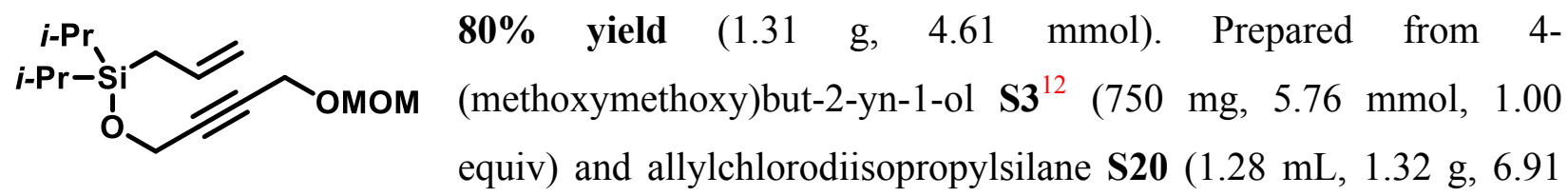
mmol, 1.20 equiv).

$\mathbf{R}_{f}: 0.8$ (silica, 1\% EtOAc/hexanes).

${ }^{1}$ H NMR (400 MHz, $\left.\mathbf{C D C l}_{3}\right): \delta 5.88$ (ddt, $\left.J=16.9,10.0,8.1 \mathrm{~Hz}, 1 \mathrm{H}\right), 4.97$ (ddt, $J=16.9,2.1,1.5$ $\mathrm{Hz}, 1 \mathrm{H}), 4.88(\mathrm{ddt}, J=10.1,2.2,1.1 \mathrm{~Hz}, 1 \mathrm{H}), 4.70(\mathrm{~s}, 2 \mathrm{H}), 4.42(\mathrm{t}, J=1.8 \mathrm{~Hz}, 2 \mathrm{H}), 4.26(\mathrm{t}, J=$ $1.8 \mathrm{~Hz}, 2 \mathrm{H}), 3.38(\mathrm{~s}, 3 \mathrm{H}), 1.75(\mathrm{dt}, J=8.0,1.3 \mathrm{~Hz}, 2 \mathrm{H}), 1.08-1.04(\mathrm{~m}, 14 \mathrm{H})$. 


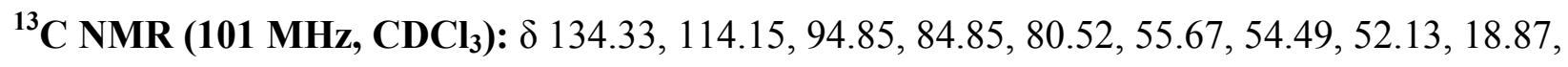
$17.56,17.54,12.61$.

IR (neat, $\mathbf{c m}^{-1}$ ): 2943, 2867, 1630, 1464, 1371, 1151, 1131, 1084, 1043, 990, 922, 882, 777.

HRMS $\left(\mathrm{CI}^{+}\right) m / z$ calculated for $\mathrm{C}_{15} \mathrm{H}_{27} \mathrm{OSi}[\mathrm{M}-\mathrm{H}]^{+} 283.17240$, found 283.17248.

\section{Allyldiisopropyl((3-phenylprop-2-yn-1-yl)oxy)silane (1f)}

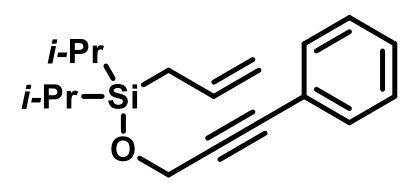

85\% yield (4.61 g, $16.1 \mathrm{mmol})$. Prepared from 3-phenylprop-2-yn-1-ol S24 (2.50 g, 18.9 mmol, 1.00 equiv) and allylchlorodiisopropylsilane S20 (4.21 mL, $4.33 \mathrm{~g}, 22.7 \mathrm{mmol}, 1.20$ equiv).

$\mathbf{R}_{f}: 0.8$ (silica, 1\% EtOAc/hexanes).

${ }^{1}$ H NMR (400 MHz, $\mathbf{C D C l}_{3}$ ): $\delta 7.50-7.45$ (m, 2H), $\delta 7.36-7.31$ (m, 3H), 5.98 (ddt, $J=16.9$, $10.1,8.0 \mathrm{~Hz}, 1 \mathrm{H}), 5.05$ (ddt, $J=16.9,2.2,1.5 \mathrm{~Hz}, 1 \mathrm{H}), 4.94$ (ddt, $J=10.1,2.2,1.1 \mathrm{~Hz}, 1 \mathrm{H}), 4.64$ $(\mathrm{s}, 2 \mathrm{H}), 1.86(\mathrm{dt}, J=8.0,1.3 \mathrm{~Hz}, 2 \mathrm{H}), 1.18-1.12(\mathrm{~m}, 14 \mathrm{H})$.

${ }^{13}$ C NMR (101 MHz, $\left.\mathbf{C D C l}_{3}\right): \delta$ 134.37, 131.68, 128.34, 128.32, 123.08, 114.11, 114.08, 87.89, $84.93,52.60,18.97,17.58,17.56,12.69$.

IR (neat, $\mathrm{cm}^{-1}$ ): 3076, 2943, 2893, 2866, 2244, 1630, 1599, 1490, 1463, 1443, 1419, 1369, 1259, 1159, 1086, 1069, 994,962, 882, 775, 754, 690, 658.

HRMS (CI $\left.\mathbf{C I}^{+} \boldsymbol{m} / \boldsymbol{z}\right)$ : calculated for $\mathrm{C}_{15} \mathrm{H}_{26} \mathrm{OSi}[\mathrm{M}+\mathrm{H}]^{+}$263.14618, found 263.14632 .

\section{Allyldiisopropyl((3-(4-methoxyphenyl)prop-2-yn-1-yl)oxy)silane (1g)}

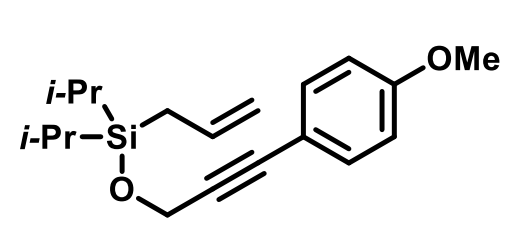

76\% yield (1.48 g, $4.68 \mathrm{mmol})$. Prepared from 3-(4methoxyphenyl)prop-2-yn-1-ol $\mathbf{S 4}^{13}$ (1.00 g, $6.17 \mathrm{mmol}, 1.00$ equiv) and allylchlorodiisopropylsilane $\mathbf{S 2 0}(1.37 \mathrm{~mL}, 1.41 \mathrm{~g}, 7.40$ mmol, 1.20 equiv).

$\mathbf{R}_{f}: 0.6$ (silica, 1\% EtOAc /hexanes).

${ }^{1}$ H NMR (400 MHz, $\left.\mathbf{C D C l}_{3}\right): \delta 7.40-7.34(\mathrm{~m}, 2 \mathrm{H}), 6.86-6.81(\mathrm{~m}, 2 \mathrm{H}), 5.94(\mathrm{ddt}, J=16.9$, $10.1,8.1 \mathrm{~Hz}, 1 \mathrm{H}), 5.00$ (ddt, $J=16.9,2.2,1.2 \mathrm{~Hz}, 1 \mathrm{H}), 4.90$ (ddt, $J=10.1,2.2,1.1 \mathrm{~Hz}, 1 \mathrm{H}), 4.59$ (s, 2H), $3.80(\mathrm{~s}, 3 \mathrm{H}), 1.81(\mathrm{dt}, J=8.0,1.3 \mathrm{~Hz}, 2 \mathrm{H}), 1.18-1.04(\mathrm{~m}, 14 \mathrm{H})$.

${ }^{13}$ C NMR (101 MHz, $\left.\mathbf{C D C l}_{3}\right): \delta$ 159.72, 134.51, 133.19, 115.21, 114.06, 114.02, 86.49, 84.87, 55.38, 52.70, 19.01, 17.62, 17.60, 12.72 .

IR (neat, $\mathbf{c m}^{-1}$ ): 2942, 2865, 1629, 1607, 1569, 1509, 1463, 1442, 1418, 1368, 1323, 1291, 1247 , $1172,1084,1066,1034,995,964,932,882,830,799,775,749,659,618,563,535$. 
HRMS (CI $\left.{ }^{+}, \boldsymbol{m} / \boldsymbol{z}\right)$ : calculated for $\mathrm{C}_{19} \mathrm{H}_{29} \mathrm{O}_{2} \mathrm{Si}[\mathrm{M}+\mathrm{H}]^{+}$317.19313, found 317.19334.

4-(3-((Allyldiisopropylsilyl)oxy)prop-1-yn-1-yl)benzonitrile (1h)

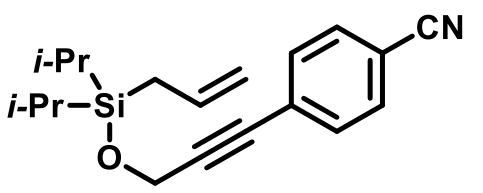

73\% yield (1.45 g, 4.65 mmol). Prepared from 4-(3-hydroxyprop1-yn-1-yl)benzonitrile $\mathbf{S 5}^{14}$ (1.00 g, $6.36 \mathrm{mmol}, 1.00$ equiv) and allylchlorodiisopropylsilane $\mathbf{S 2 0}$ (2.31 mL, $2.38 \mathrm{~g}, 7.63 \mathrm{mmol}, 1.20$

equiv).

$\mathbf{R}_{f}: 0.65$ (silica, 1\% EtOAc/hexanes).

${ }^{1}$ H NMR (400 MHz, $\left.\mathbf{C D C l}_{3}\right): \delta 7.62-7.58(\mathrm{~m}, 2 \mathrm{H}), 7.52-7.48(\mathrm{~m}, 2 \mathrm{H}), 5.91$ (ddt, $J=16.9$, $10.0,8.1 \mathrm{~Hz}, 1 \mathrm{H}), 5.00$ (ddt, $J=16.9,2.1,1.2 \mathrm{~Hz}, 1 \mathrm{H}), 4.90$ (ddt, $J=10.0,2.1,1.1 \mathrm{~Hz}, 1 \mathrm{H}), 4.62$ (s, 2H), $1.79(\mathrm{dt}, J=8.1,1.3 \mathrm{~Hz}, 2 \mathrm{H}), 1.13-1.05$ (m, 14H).

${ }^{13}$ C NMR (101 MHz, $\left.\mathbf{C D C l}_{3}\right): \delta 134.17,132.22,132.16,132.13,127.95,118.52,114.32,111.83$, 92.54, 83.34, 52.52, 18.87, 17.56, 17.54, 12.64 .

IR (neat, $\mathbf{c m}^{-1}$ ): 2943, 2866, 2229, 1738, 1630, 1605, 1500, 1463, 1369, 1261, 1160, 1088, 994, 961, 932, 882, 839, 776, 751, 659, 617, 554.

HRMS (CI $\left.{ }^{+}, \boldsymbol{m} / \boldsymbol{z}\right)$ : calculated for $\mathrm{C}_{19} \mathrm{H}_{26} \mathrm{NOSi}[\mathrm{M}+\mathrm{H}]^{+}$312.178986, found 312.178368.

\section{Methyl 4-(3-((allyldiisopropylsilyl)oxy)prop-1-yn-1-yl)benzoate (1i)}

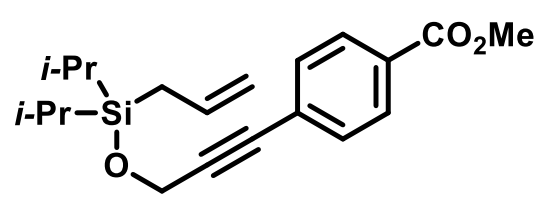

78\% yield (1.41 g, $4.09 \mathrm{mmol})$. Prepared from methyl 4-(3hydroxyprop-1-yn-1-yl)benzoate $\mathbf{S 6}^{15}$ (1.00 g , $5.26 \mathrm{mmol}, 1.00$ equiv) and allylchlorodiisopropylsilane $\mathbf{S 2 0}(1.17 \mathrm{~mL}, 1.20 \mathrm{~g}$, $6.31 \mathrm{mmol}, 1.20$ equiv).

$\mathbf{R}_{f}: 0.55$ (silica, 1\% EtOAc/hexanes).

${ }^{1}$ H NMR (400 MHz, $\left.\mathbf{C D C l}_{3}\right): \delta 8.00-7.93(\mathrm{~m}, 2 \mathrm{H}), 7.50-7.42$ (m, 2H), 5.90 (ddt, $J=17.0$, $10.1,8.1 \mathrm{~Hz}, 1 \mathrm{H}), 4.99$ (ddt, $J=16.9,2.2,1.6 \mathrm{~Hz}, 1 \mathrm{H}), 4.89$ (ddt, $J=10.1,2.2,1.1 \mathrm{~Hz}, 1 \mathrm{H}), 4.60$ (s, 2H), $3.89(\mathrm{~s}, 3 \mathrm{H}), 1.79(\mathrm{dt}, J=8.1,1.3 \mathrm{~Hz}, 2 \mathrm{H}), 1.11-1.06(\mathrm{~m}, 14 \mathrm{H})$.

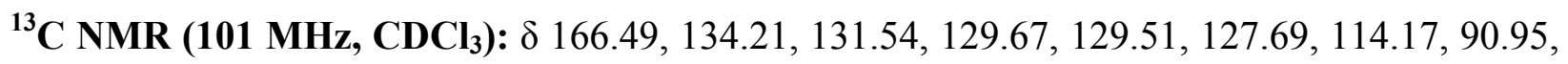
$84.15,52.52,52.23,18.86,17.52,17.50,12.62$.

IR (neat, $\mathbf{c m}^{-1}$ ): 2945, 2866, 1725, 1630, 1606, 1506, 1463, 1436, 1406, 1367, 1307, 1272, 1192, 1176, 1090, 1019, 993, 966, 932, 882, 857, 827, 768, 738, 695, 659, 620, 527.

HRMS $\left(\mathbf{C I}^{+}, \boldsymbol{m} / \boldsymbol{z}\right)$ : calculated for $\mathrm{C}_{20} \mathrm{H}_{27} \mathrm{O}_{3} \mathrm{Si}[\mathrm{M}-\mathrm{H}]^{+}$343.171438, found 343.172948. 


\section{Allyldiisopropyl((3-(4-(trifluoromethyl)phenyl)prop-2-yn-1-yl)oxy)silane (1j)}

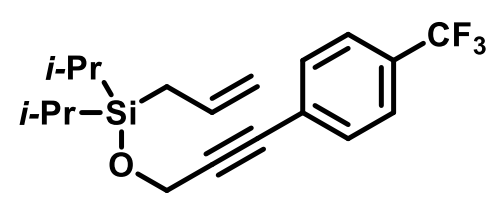

73\% yield (649 mg, $1.83 \mathrm{mmol})$. Prepared from 3-(4(trifluoromethyl)phenyl)prop-2-yn-1-ol $\mathbf{S} 7^{16}$ (500 mg, $2.50 \mathrm{mmol}$, 1.00 equiv) and allylchlorodiisopropylsilane $\mathbf{S 2 0}(0.56 \mathrm{~mL}, 572 \mathrm{mg}$, $3.00 \mathrm{mmol}, 1.20$ equiv).

$\mathbf{R}_{\boldsymbol{f}}: 0.7$ (silica, 1\% EtOAc/hexanes).

${ }^{1}$ H NMR (400 MHz, $\left.\mathbf{C D C l}_{3}\right): \delta 7.60-7.50$ (m, 4H), $5.93(\mathrm{ddt}, J=17.0,10.0,8.0 \mathrm{~Hz}, 1 \mathrm{H}), 5.01$ (ddt, $J=17.0,2.2,1.2 \mathrm{~Hz}, 1 \mathrm{H}), 4.91$ (ddt, $J=10.0,2.2,1.1 \mathrm{~Hz}, 1 \mathrm{H}), 4.62$ (s, 2H), 1.81 (dt, $J=8.0$, $1.3 \mathrm{~Hz}, 2 \mathrm{H}), 1.13-1.07$ (m, 14H).

${ }^{13}$ C NMR (101 MHz, CDCl $\left.\mathbf{3}\right): \delta$ 134.29, 131.96, 130.68, 130.35, 130.03, 129.71, 125.42, 125.40, $125.38,125.34,125.31,114.26,90.50,83.63,52.56,18.93,17.58,17.56,12.69 .\left({ }^{1} \mathrm{~J}_{\mathrm{C}-\mathrm{F}}=130.68\right.$, 130.35.130.03, 129.71)

IR (neat, $\mathbf{c m}^{-1}$ ): 2945, 2867, 1630, 1617, 1463, 1405, 1367, 1322, 1264, 1167, 1128, 1104, 1065, 1018, 995, 964, 932, 897, 882, 842, 776, 740, 713, 659, 619, 597, 521.

HRMS (CI $\left.{ }^{+}, \boldsymbol{m} / \boldsymbol{z}\right)$ : calculated for $\mathrm{C}_{19} \mathrm{H}_{26} \mathrm{~F}_{3} \mathrm{OSi}[\mathrm{M}+\mathrm{H}]^{+}$355.16995, found 355.17023.

\section{Allyldiisopropyl((6-methylhept-2-yn-4-yl)oxy)silane (5a)}

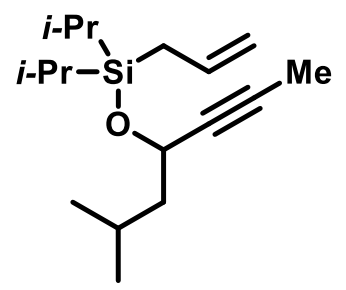

73\% yield (813 mg, $2.90 \mathrm{mmol}$ ). Prepared from 6-methylhept-2-yn-4-ol $\mathbf{S 8}^{17}$ (500 mg, $3.97 \mathrm{mmol}, 1.00$ equiv) and allylchlorodiisopropylsilane $\mathbf{S 2 0}$ (0.89 mL, $909 \mathrm{mg}, 4.76 \mathrm{mmol}, 1.20$ equiv).

$\mathbf{R}_{f}: 0.7$ (silica, 1\% EtOAc/hexanes).

${ }^{1}$ H NMR (400 MHz, $\left.\mathbf{C D C l}_{3}\right)$ : $\delta 5.91$ (ddt, $\left.J=16.9,10.1,8.0 \mathrm{~Hz}, 1 \mathrm{H}\right), 4.96(\mathrm{ddt}, J=17.0,2.6,1.3$ $\mathrm{Hz}, 1 \mathrm{H}), 4.85$ (ddt, $J=10.1,2.2,1.1 \mathrm{~Hz}, 1 \mathrm{H}), 4.45$ (tq, $J=7.2,3.9 \mathrm{~Hz}, 1 \mathrm{H}), 1.82$ (dd, $J=8.9,2.2$ $\mathrm{Hz}, 3 \mathrm{H}), 1.77(\mathrm{dt}, J=8.1,1.3 \mathrm{~Hz}, 2 \mathrm{H}), 1.61-1.46(\mathrm{~m}, 2 \mathrm{H}), 1.09-1.02(\mathrm{~m}, 15 \mathrm{H}), 0.90(\mathrm{dd}, J=$ $8.9,6.6,0.8 \mathrm{~Hz}, 6 \mathrm{H})$.

${ }^{13}$ C NMR (101 MHz, $\left.\mathbf{C D C l}_{3}\right): \delta$ 135.00, 113.67, 81.36, 80.25, 62.09, 48.22, 24.74, 22.87, 22.72, $19.51,17.76,17.68,17.67,17.65,13.03,12.98,3.65$.

IR (neat, $\mathbf{c m}^{-1}$ ): 2957, 2867, 1630, 1465, 1386, 1159, 1064, 994, 883, 851, 747.

HRMS (CI, $\boldsymbol{m} / \boldsymbol{z})$ : calculated for $\mathrm{C}_{17} \mathrm{H}_{33} \mathrm{OSi}[\mathrm{M}+\mathrm{H}]^{+}$281.22952, found 281.22961. 


\section{Allyl(hept-2-yn-4-yloxy)diisopropylsilane (5b)}

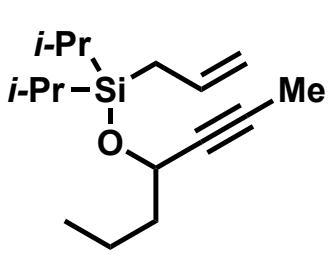

73\% yield (607 mg, 2.28 mmol). Prepared from hept-2-yn-4-ol S9 ${ }^{18}$ (350 $\mathrm{mg}, 3.12 \mathrm{mmol}, 1.00$ equiv) and allylchlorodiisopropylsilane $\mathbf{S 2 0}(0.70 \mathrm{~mL}$, $714 \mathrm{mg}, 3.74 \mathrm{mmol}, 1.20$ equiv).

$\mathbf{R}_{\boldsymbol{f}}: 0.7$ (silica, 1\% EtOAc/hexanes).

${ }^{1}$ H NMR (400 MHz, $\left.\mathbf{C D C l}_{3}\right)$ : $\delta 5.91$ (ddt, $\left.J=17.0,10.0,8.1 \mathrm{~Hz}, 1 \mathrm{H}\right), 4.96(\mathrm{ddt}, J=16.9,2.2,1.5$ $\mathrm{Hz}, 1 \mathrm{H}), 4.85$ (ddt, $J=10.0,2.2,1.2 \mathrm{~Hz}, 1 \mathrm{H}), 4.40$ (tq, $J=6.4,2.1 \mathrm{~Hz}, 1 \mathrm{H}), 1.82$ (d, $J=2.1 \mathrm{~Hz}$, $3 \mathrm{H}), 1.79-1.75(\mathrm{~m}, 2 \mathrm{H}), 1.66-1.58(\mathrm{~m}, 2 \mathrm{H}), 1.49-1.39(\mathrm{~m}, 2 \mathrm{H}), 1.08-1.02(\mathrm{~m}, 14 \mathrm{H}), 0.92(\mathrm{t}$, $J=7.4 \mathrm{~Hz}, 3 \mathrm{H})$.

${ }^{13}$ C NMR (101 MHz, $\left.\mathbf{C D C l}_{3}\right): \delta$ 135.02, 113.66, 81.26, 80.14, 63.30, 41.37, 19.43, 18.62, 17.74, $17.65,17.63,14.02,12.98,12.92,3.67$.

IR (neat, cm$^{-1}$ ): 2963, 2865, 2946, 1631, 1464, 1390, 1348, 1157, 1110, 1065, 1038, 883, 762.

HRMS (CI', $\boldsymbol{m} / \boldsymbol{z})$ : calculated for $\mathrm{C}_{16} \mathrm{H}_{30} \mathrm{OSi}[\mathrm{M}+]$ 266,206594, found 266.206481.

\section{Allyldiisopropyl((5-methyl-1-phenylhex-1-yn-3-yl)oxy)silane (5c)}

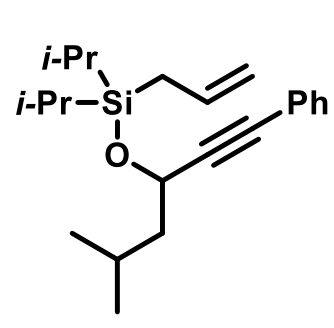

81\% yield (730 mg, $2.13 \mathrm{mmol}$ ). Prepared from 5-methyl-1-phenylhex-1yn-3-ol $\quad$ S10 $^{19} \quad(500 \quad \mathrm{mg}, \quad 2.66 \quad \mathrm{mmol}, \quad 1.00 \quad$ equiv $)$ and allylchlorodiisopropylsilane $\mathbf{S 2 0}(0.59 \mathrm{~mL}, 609 \mathrm{mg}, 3.19 \mathrm{mmol}, 1.20$ equiv).

$\mathbf{R}_{f}: 0.7$ (silica, $1 \%$ EtOAc/hexanes).

${ }^{1}$ H NMR (400 MHz, $\left.\mathbf{C D C l}_{3}\right): \delta 7.43-7.37$ (m, 2H), 7.33 - 7.27 (m, 3H), 5.94 (ddt, $J=16.9$, $10.1,8.1 \mathrm{~Hz}, 1 \mathrm{H}), 4.98$ (ddt, $J=16.9,2.2,1.5 \mathrm{~Hz}, 1 \mathrm{H}), 4.87$ (ddt, $J=10.0,2.2,1.2 \mathrm{~Hz}, 1 \mathrm{H}), 4.72$ $(\mathrm{t}, J=7.0 \mathrm{~Hz}, 1 \mathrm{H}), 1.92(\mathrm{pd}, J=6.8,5.3 \mathrm{~Hz}, 1 \mathrm{H}), 1.83(\mathrm{dq}, J=8.2,1.4 \mathrm{~Hz}, 2 \mathrm{H}), 1.13-1.04(\mathrm{~m}$, $16 \mathrm{H}), 0.98-0.93(\mathrm{~m}, 6 \mathrm{H})$.

${ }^{13}$ C NMR (101 MHz, $\left.\mathbf{C D C l}_{3}\right): \delta$ 134.86, 131.62, 128.39, 128.21, 123.33, 113.88, 90.41, 84.40, $62.35,47.94,24.82,22.86,22.84,19.50,17.79,17.73,17.70,13.05,13.00$.

IR (neat, $\mathbf{c m}^{-1}$ ): 2956, 2867, 1630, 1492, 1462, 1084, 754, 880.

HRMS (CI, $\boldsymbol{m} / \mathbf{z})$ : calculated for $\mathrm{C}_{22} \mathrm{H}_{35} \mathrm{OSi}[\mathrm{M}+\mathrm{H}]^{+}$343.24517, found 343.24537. 


\section{Allyl((1,3-diphenylprop-2-yn-1-yl)oxy)diisopropylsilane (5d)}

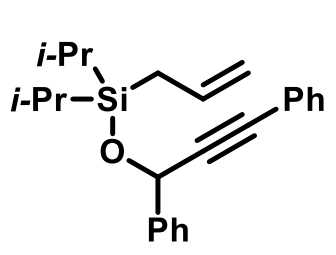

84\% yield (732 mg, $2.02 \mathrm{mmol}$ ). Prepared from 1,3-diphenylprop-2-yn-1ol S11 ${ }^{20}$ (500 mg, $2.40 \mathrm{mmol}, 1.00$ equiv) and allylchlorodiisopropylsilane S20 (0.54 mL, $550 \mathrm{mg}, 2.88 \mathrm{mmol}, 1.20$ equiv).

$\mathbf{R}_{f}: 0.7$ (silica, $1 \%$ EtOAc/hexanes).

${ }^{1}$ H NMR (400 MHz, $\left.\mathbf{C D C l}_{3}\right): \delta 7.60$ - $7.56(\mathrm{~m}, 2 \mathrm{H}), 7.46-7.35$ (m, 4H), $7.34-7.28(\mathrm{~m}, 4 \mathrm{H})$, 5.94 (ddt, $J=16.5,10.0,8.1 \mathrm{~Hz}, 1 \mathrm{H}), 5.82$ (s, 1H), 4.99 (ddt, $J=16.9,2.2,1.5 \mathrm{~Hz}, 1 \mathrm{H}), 4.88$ (ddt, $J=10.0,2.1,1.0 \mathrm{~Hz}, 1 \mathrm{H}), 1.89(\mathrm{dt}, J=8.1,1.3 \mathrm{~Hz}, 2 \mathrm{H}), 1.16-1.05(\mathrm{~m}, 14 \mathrm{H})$.

${ }^{13}$ C NMR (101 MHz, CDCl $)$ ): $\delta$ 141.90, 134.60, 131.72, 128.46, 128.39, 127.83, 126.25, 122.99, $114.13,90.34,85.72,65.41,19.42,17.75,17.72,17.70,13.00,12.98$.

IR (neat, $\mathbf{c m}^{-1}$ ): 3073, 2944, 2867, 1694, 1630, 1599, 1492, 1451, 1273, 1060, 885, 755, 692.

HRMS (CI $\left.{ }^{+}, \boldsymbol{m} / \boldsymbol{z}\right)$ : calculated for $\mathrm{C}_{24} \mathrm{H}_{31} \mathrm{OSi}[\mathrm{M}+\mathrm{H}]^{+} 363.21387$, found 363.21418 .

\section{Allyl((1,4-diphenylbut-3-yn-2-yl)oxy)diisopropylsilane (5e)}

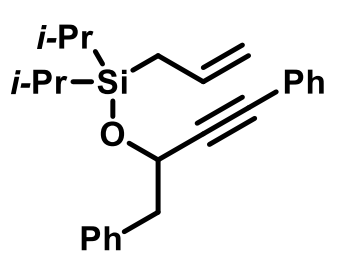

81\% yield (1.37 g, $3.64 \mathrm{mmol})$. Prepared from 1,4-diphenylbut-3-yn-2-ol $\mathbf{S 1 2}^{21}$ (1.00 g, $4.50 \mathrm{mmol}, 1.00$ equiv) and allylchlorodiisopropylsilane $\mathbf{S 2 0}$ (1.00 mL, $1.03 \mathrm{~g}, 5.4 \mathrm{mmol}, 1.20$ equiv). $\mathbf{R}_{f}: 0.7$ (silica, 1\% EtOAc/hexanes).

${ }^{1}$ H NMR (400 MHz, $\left.\mathbf{C D C l}_{3}\right): \delta 7.39-7.21(\mathrm{~m}, 10 \mathrm{H}), 5.83(\mathrm{ddt}, J=17.0,10.0,8.1 \mathrm{~Hz}, 1 \mathrm{H}), 4.93$ (ddt, $J=16.9,2.2,1.5 \mathrm{~Hz}, 1 \mathrm{H}), 4.84(\mathrm{ddt}, J=10.0,2.2,1.5 \mathrm{~Hz}, 1 \mathrm{H}), 4.82(\mathrm{~d}, J=6.6 \mathrm{~Hz}, 1 \mathrm{H}), 3.07$ $(\mathrm{dd}, J=7.0,0.4 \mathrm{~Hz}, 2 \mathrm{H}), 1.71$ (ddt, $J=8.1,5.4,1.3 \mathrm{~Hz}, 2 \mathrm{H}), 1.11-0.95$ (m, 14H).

${ }^{13}$ C NMR (101 MHz, $\left.\mathbf{C D C l}_{3}\right): \delta 137.57,134.74,131.57,130.17,128.39,128.32,128.19,126.70$, $123.09,113.88,90.66,85.24,65.01,45.47,19.25,17.63,17.58,12.89,12.85$.

IR (neat, $\mathbf{~ c m}^{-1}$ ): 3032, 2944, 2866, 1630, 1490, 1463, 1342, 1266, 1159, 1083, 993, 882, 754, 690. HRMS (CI, $\boldsymbol{m} / \boldsymbol{z})$ : calculated for $\mathrm{C}_{25} \mathrm{H}_{33} \mathrm{OSi}[\mathrm{M}+\mathrm{H}]^{+}$377.22952, found 377.22973.

Allyl((1,5-diphenylpent-1-yn-3-yl)oxy)diisopropylsilane (5f)

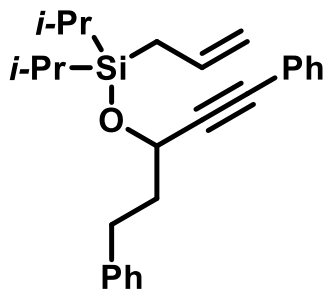

82\% yield (1.02 g, 2.60 mmol). Prepared from 1,5-diphenylpent-1-yn-3-ol $\mathbf{S 1 3}^{22}(0.71 \mathrm{~mL}, 750 \mathrm{mg}, 3.17 \mathrm{mmol}, 1.00$ equiv) and allylchlorodiisopropylsilane $\mathbf{S 2 0}$ (725 mg, $3.80 \mathrm{mmol}, 1.20$ equiv). $\mathbf{R}_{f}: 0.7$ (silica, 1\% EtOAc/hexanes). 
${ }^{1}$ H NMR (400 MHz, $\left.\mathbf{C D C l}_{3}\right): \delta 7.46-7.40(\mathrm{~m}, 2 \mathrm{H}), 7.35-7.15(\mathrm{~m}, 8 \mathrm{H}), 5.93$ (ddt, $J=17.0$, 10.1, 2.0 Hz, 1H), 4.97 (ddt, $J=16.9,3.7,2.2 \mathrm{~Hz}, 1 \mathrm{H}), 4.87$ (ddt, $J=10.0,2.1,1.0 \mathrm{~Hz}, 1 \mathrm{H}), 4.72$ $(\mathrm{td}, J=6.3,1.9 \mathrm{~Hz}, 1 \mathrm{H}), 2.90-2.82(\mathrm{~m}, 2 \mathrm{H}), 2.15-2.07(\mathrm{~m}, 2 \mathrm{H}), 1.85-1.80(\mathrm{~m}, 2 \mathrm{H}), 1.14-$ $1.05(\mathrm{~m}, 14 \mathrm{H})$.

${ }^{13}$ C NMR (101 MHz, $\left.\mathbf{C D C l}_{3}\right): \delta$ 141.92, 134.76, 131.66, 128.64, 128.52, 128.43, 128.34, 125.99, 123.16, 113.93, 90.77, 84.79, 63.11, 40.56, 31.63, 19.41, 17.77, 17.72, 17.69, 13.02, 12.96 .

IR (neat, $\mathbf{~ c m}^{-1}$ ): 3027, 2943, 2865, 1630, 1602, 1490, 1462, 1340, 1159, 1090, 991, 882, 843, 754, 690.

HRMS (CI $\left.{ }^{+}, \boldsymbol{m} / \boldsymbol{z}\right)$ : calculated for $\mathrm{C}_{26} \mathrm{H}_{35} \mathrm{OSi}[\mathrm{M}+\mathrm{H}]^{+}$391.24517, found 391.24552.

\section{Allyldiisopropyl((1-phenylhex-5-en-1-yn-3-yl)oxy)silane (5g)}

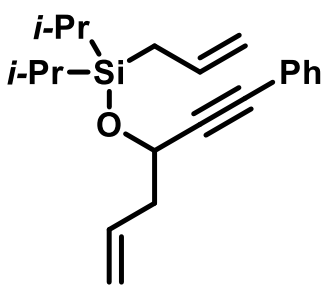

80\% yield (3.79 g, 11.6 mmol). Prepared from 1-phenylhex-5-en-1-yn-3-ol $\mathbf{S 1 4}^{23}$ (2.50 g, $14.5 \mathrm{mmol}, 1.00$ equiv) and allylchlorodiisopropylsilane $\mathbf{S 2 0}$ (3.23 mL, $3.32 \mathrm{~g}, 17.4 \mathrm{mmol}, 1.20$ equiv).

$\mathbf{R}_{f}: 0.8$ (silica, $1 \%$ EtOAc/hexanes).

${ }^{1}$ H NMR (400 MHz, $\left.\mathbf{C D C l}_{3}\right): \delta 7.46-7.40(\mathrm{~m}, 2 \mathrm{H}), 7.34-7.29(\mathrm{~m}, 3 \mathrm{H}), 6.03-5.90(\mathrm{~m}, 2 \mathrm{H})$, $5.23-5.13(\mathrm{~m}, 2 \mathrm{H}), 5.01(\mathrm{ddt}, J=16.9,2.3,1.2 \mathrm{~Hz}, 1 \mathrm{H}), 4.90(\mathrm{dt}, J=10.1,2.2,1.2 \mathrm{~Hz}, 1 \mathrm{H}), 4.73$ $(\mathrm{dt}, J=6.4,1.1 \mathrm{~Hz}, 1 \mathrm{H}), 2.56(\mathrm{dt}, J=7.3,2.3 \mathrm{~Hz}, 2 \mathrm{H}), 1.85(\mathrm{dt}, J=8.0,1.3 \mathrm{~Hz}, 2 \mathrm{H}), 1.15-1.06$ (m, 14H).

${ }^{13}$ C NMR (101 MHz, CDCl $)$ : $\delta$ 134.73, 134.07, 131.67, 128.38, 128.30, 123.16, 117.87, 113.95, 90.62, 84.84, 63.54, 43.51, 19.38, 17.73, 17.67, 17.65, 12.98, 12.93 .

IR (neat, $\mathbf{c m}^{-1}$ ): 3079, 2943, 2866, 1630, 1490, 1463, 1342, 1159, 1084, 993, 914, 882, 754, 689. HRMS $\left(\mathbf{C I}^{+}, \boldsymbol{m} / \boldsymbol{z}\right)$ : calculated for $\mathrm{C}_{21} \mathrm{H}_{31} \mathrm{OSi}[\mathrm{M}+\mathrm{H}]^{+}$327.21387, found 327.21409.

\section{Allyldiisopropyl((1-phenylhex-1-yn-3-yl)oxy)silane (5h)}

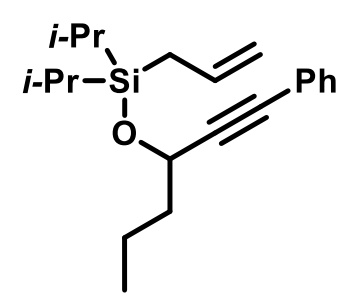

79\% yield (1.34 g, 4.08 mmol). Prepared from 1-phenylhex-1-yn-3-ol S15 $\mathbf{S H}^{24}$ (750 mg, $4.3 \mathrm{mmol}, 1.00$ equiv) and allylchlorodiisopropylsilane $\mathbf{S 2 0}$ (0.96 $\mathrm{mL}, 85 \mathrm{mg}, 5.16 \mathrm{mmol}, 1.20$ equiv). $\mathbf{R}_{f}: 0.7$ (silica, 1\% EtOAc/hexanes).

${ }^{1}$ H NMR (400 MHz, $\left.\mathbf{C D C l}_{3}\right): \delta 7.43-7.38$ (m, 2H), $7.32-7.28$ (m, 3H), 5.94 (ddt, $J=16.9$, $10.0,8.1 \mathrm{~Hz}, 1 \mathrm{H}), 4.98$ (ddt, $J=16.9,2.1,1.5 \mathrm{~Hz}, 1 \mathrm{H}), 4.87$ (ddt, $J=10.0,2.2,1.1 \mathrm{~Hz}, 1 \mathrm{H}), 4.68$ 
$(\mathrm{t}, J=6.4 \mathrm{~Hz}, 1 \mathrm{H}), 1.82(\mathrm{dt}, J=8.1,1.4 \mathrm{~Hz}, 2 \mathrm{H}), 1.79-1.72(\mathrm{~m}, 2 \mathrm{H}), 1.58-1.47(\mathrm{~m}, 2 \mathrm{H}), 1.16$ $-1.03(\mathrm{~m}, 14 \mathrm{H}), 0.96(\mathrm{t}, J=7.4 \mathrm{~Hz}, 3 \mathrm{H})$.

${ }^{13}$ C NMR (101 MHz, $\left.\mathbf{C D C l}_{3}\right): \delta$ 134.86, 131.64, 128.38, 128.20, 123.34, 113.85, 91.31, 84.30, 63.58, 41.13, 19.44, 18.68, 17.77, 17.71, 17.70, 17.68, 14.05, 13.03, 12.97 .

IR (neat, $\mathbf{c m}^{-1}$ ): 2960, 2942, 2866, 1630, 1490, 1463, 1341, 1111, 1082, 1038, 994, 882, 754, 689. HRMS (CI, $\boldsymbol{m} / \boldsymbol{z})$ : calculated for $\mathrm{C}_{21} \mathrm{H}_{33} \mathrm{OSi}[\mathrm{M}+\mathrm{H}]^{+}$329.22952, found 329.22952.

\section{Allyldiisopropyl((1-phenyloct-7-en-1-yn-3-yl)oxy)silane (5i)}

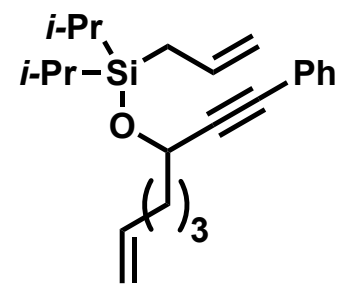

73\% yield (1.03 g, $2.91 \mathrm{mmol})$. Prepared from 1-phenyloct-7-en-1-yn-3-ol $\mathbf{S 1 6}^{25}$ (800 mg, 3.99 mmol, 1.00 equiv) and allylchlorodiisopropylsilane $\mathbf{S 2 0}$ (0.89 mL, $914 \mathrm{mg}, 4.79 \mathrm{mmol}, 1.20$ equiv).

$\mathbf{R}_{f}: 0.5$ (silica, 1\% EtOAc/hexanes).

${ }^{1}$ H NMR (400 MHz, CDCl 3 ): $\delta 7.43-7.38(\mathrm{~m}, 2 \mathrm{H}), 7.32-7.28(\mathrm{~m}, 3 \mathrm{H}), 6.00-5.77$ (m, 2H), $5.07-4.99(\mathrm{~m}, 2 \mathrm{H}), 4.97(\mathrm{dt}, J=2.2,1.2 \mathrm{~Hz}, 1 \mathrm{H}), 4.87(\mathrm{ddt}, J=10.0,2.2,1.1 \mathrm{~Hz}, 1 \mathrm{H}), 4.69$ (t, $J$ $=6.3 \mathrm{~Hz}, 1 \mathrm{H}), 2.12(\mathrm{tdd}, J=14.1,6.9,1.4 \mathrm{~Hz}, 2 \mathrm{H}), 1.82(\mathrm{dt}, J=8.1,1.3 \mathrm{~Hz}, 2 \mathrm{H}), 1.81-1.76(\mathrm{~m}$, 2H), $1.66-1.57(\mathrm{~m}, 2 \mathrm{H}), 1.12-1.05(\mathrm{~m}, 14 \mathrm{H})$.

${ }^{13}$ C NMR (101 MHz, $\left.\mathbf{C D C l}_{3}\right): \delta$ 138.83, 134.83, 131.64, 128.40, 128.24, 123.27, 114.75, 113.89, $91.14,84.41,63.62,38.34,33.58,24.62,19.42,17.77,17.70,17.69,13.01,12.96$.

IR (neat, $\mathbf{c m}^{-1}$ ): 3078, 2943, 2866, 1630, 1490, 1461, 1091, 882, 754.

HRMS (CI ${ }^{+}, \boldsymbol{m} / \boldsymbol{z}$ ): calculated for $\mathrm{C}_{24} \mathrm{H}_{35} \mathrm{OSi}[\mathrm{M}+\mathrm{H}]^{+}$355.24517, found 355.24503 .

\section{Allyldiisopropyl((1-phenyloct-1-yn-3-yl)oxy)silane (5j)}

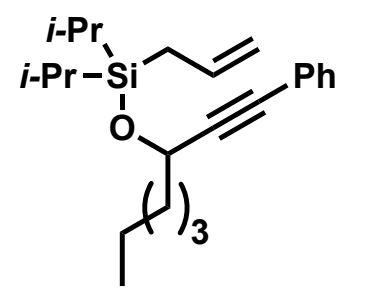

74\% yield (785 mg, $2.20 \mathrm{mmol}$ ). Prepared from 1-phenyloct-1-yn-3-ol $\mathbf{S 1 7 ^ { 2 6 }}$ (600 mg, $2.97 \mathrm{mmol}, 1.00$ equiv) and allylchlorodiisopropylsilane $\mathbf{S 2 0}$ (0.66 mL, $680 \mathrm{mg}, 3.56 \mathrm{mmol}, 1.20$ equiv).

$\mathbf{R}_{f}: 0.7$ (silica, 1\% EtOAc/hexanes).

${ }^{1}$ H NMR (400 MHz, $\left.\mathbf{C D C l}_{3}\right): \delta 7.49$ - 7.41 (m, 2H), 7.36 - 7.29 (m, 3H), 5.99 (ddt, $J=16.9,8.6$, $1.5 \mathrm{~Hz}, 1 \mathrm{H}), 5.03(\mathrm{dt}, J=16.9,1.8 \mathrm{~Hz}, 1 \mathrm{H}), 4.92(\mathrm{dt}, J=10.1,1.8 \mathrm{~Hz}, 1 \mathrm{H}), 4.72(\mathrm{td}, J=6.5,1.6$ $\mathrm{Hz}, 1 \mathrm{H}), 1.87(\mathrm{dd}, J=8.1,1.5 \mathrm{~Hz}, 2 \mathrm{H}), 1.85-1.78(\mathrm{~m}, 2 \mathrm{H}), 1.61-1.51(\mathrm{~m}, 2 \mathrm{H}), 1.43-1.34(\mathrm{~m}$, $4 \mathrm{H}), 1.20-1.10(\mathrm{~m}, 14 \mathrm{H}), 0.95(\mathrm{~d}, J=7.0 \mathrm{~Hz}, 3 \mathrm{H})$. 
${ }^{13}$ C NMR (101 MHz, $\left.\mathbf{C D C l}_{3}\right): \delta$ 134.79, 131.60, 131.59, 128.35, 128.16, 123.32, 113.84, 91.30, 84.30, 63.78, 38.91, 31.69, 25.03, 22.76, 19.41, 17.74, 17.69, 17.67, 17.66, 14.16, 13.00, 12.95.

IR (neat, $\mathbf{c m}^{-1}$ ): 2943, 2865, 1630, 1490, 1464, 1382, 1340, 1159, 1088, 1067, 1041, 993, 882, 754, 689, 658.

HRMS $\left(\mathbf{C I}^{+}, \boldsymbol{m} / \mathbf{z}\right)$ : calculated for $\mathrm{C}_{23} \mathrm{H}_{37} \mathrm{OSi}[\mathrm{M}+\mathrm{H}]^{+}$357.26082, found 357.26109.

Allyl(hept-1-en-5-yn-4-yloxy)diisopropylsilane (5k)

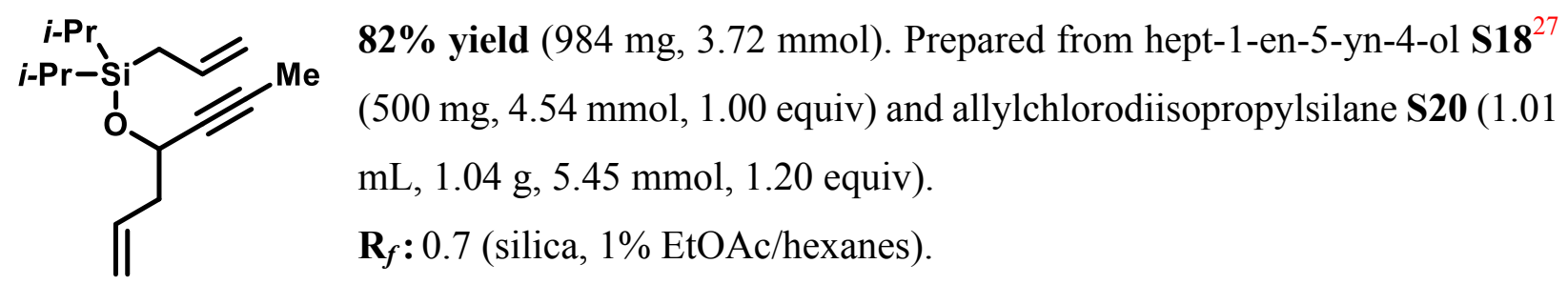

${ }^{1}$ H NMR (400 MHz, $\mathbf{C D C l}_{3}$ ): $\delta 5.96-5.82$ (m, 2H), $5.14-5.06$ (m, 2H), 4.96 (ddt, $J=16.9,2.2$, $1.5 \mathrm{~Hz}, 1 \mathrm{H}$ ), 4.86 (ddt, $J=10.0,2.2,1.1 \mathrm{~Hz}, 1 \mathrm{H}), 4.43$ (tq, $J=6.3,2.1 \mathrm{~Hz}, 1 \mathrm{H}$ ), 2.40 (ddt, $J=6.8$, $1.3 \mathrm{~Hz}, 2 \mathrm{H}), 1.83$ (d, $J=2.1 \mathrm{~Hz}, 3 \mathrm{H}), 1.77$ (dt, $J=8.1,1.3 \mathrm{~Hz}, 2 \mathrm{H}), 1.09-1.02(\mathrm{~m}, 14 \mathrm{H})$.

${ }^{13}$ C NMR (101 MHz, $\left.\mathbf{C D C l}_{3}\right): \delta$ 134.75, 134.73, 134.28, 117.45, 117.43, 113.73, 80.60, 63.20, 43.67, 19.32, 17.64, 17.55, 17.53, 12.91, 12.84, 3.56, 3.54.

IR (neat, $\mathbf{~ c m}^{-1}$ ): 3077, 2943, 2867, 1630, 1464, 1343, 1157, 1084, 991, 914, 882, 748.

HRMS $\left(\mathbf{C I}^{+}, \boldsymbol{m} / z\right)$ : calculated for $\mathrm{C}_{16} \mathrm{H}_{29} \mathrm{OSi}[\mathrm{M}+\mathrm{H}]^{+}$265.19822, found 265.19843 . 


\section{Pauson-Khand Reaction (PKR) of Siloxy-Tethered 1,7-enynes}

A. PKR General Procedure

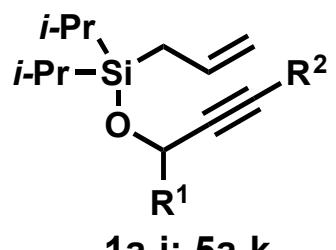

1a-j; 5a-k a) $\mathrm{Co}_{2}(\mathrm{CO})_{8}$ (1.05 equiv)

$\frac{\text { o-xylene, } 2 \mathrm{~h}, 25^{\circ} \mathrm{C}}{\text { b) } 4-\mathrm{FBnSMe}(5.0 \text { equiv })}$

$130^{\circ} \mathrm{C}, 12 \mathrm{~h}$

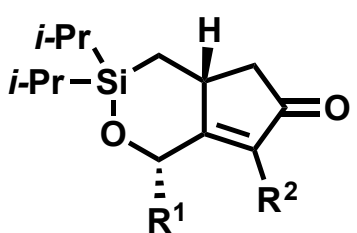

2a-j; 6a-k

General Procedure 2: Pauson-Khand Reaction of Siloxy-Tethered 1,7-enynes unless otherwise indicated (see $\mathbf{6} \boldsymbol{e}$ and $\mathbf{6 g}$ ), reactions were performed on a $0.334 \mathrm{mmol}$ scale. The isolated yields reported represent an average of three experiments.

A flame dried $30 \mathrm{~mL}$ vial equipped with a magnetic stir-bar was sequentially charged with the enyne (1a-j and 5a-k; $0.334 \mathrm{mmol}, 1.00$ equiv), $o$-xylene $(3.40 \mathrm{~mL}, 0.10 \mathrm{M}), \mathrm{Co}_{2}(\mathrm{CO})_{8}(120 \mathrm{mg}$, $0.35 \mathrm{mmol}, 1.05$ equiv), and stirred for $2 \mathrm{~h}$ at room temperature $\left(25^{\circ} \mathrm{C}\right)$ under inert atmosphere $\left(\mathrm{N}_{2}\right)$. After 2 hours, the reaction was monitored by TLC analysis to ensure full conversion of the enyne to the cobalt-alkyne complex. To the reaction mixture 4-fluorobenzyl(methyl)sulfide $\mathbf{S 2 1}{ }^{30}$ (4-FBnSMe) (261 mg, $1.67 \mathrm{mmol}, 5.00$ equiv) was added, and the vial was immediately placed in a pre-heated oil bath $\left(130^{\circ} \mathrm{C}\right)$ for $12 \mathrm{~h}$. The solvent was removed under reduced pressure and the crude reaction mixture dry loaded on silica gel. Flash chromatography (silica, 100\% hexanes) provided the recovered the promoter 4-FBnSMe S21 (83\% average recovery). Increasing the polarity of the solvent system (1\% - 10\% EtOAc/hexanes) yields $\mathbf{2 a - j}$ and $\mathbf{6 a - k}$ as a colorless oils or white to clear crystalline solids.

Note: For enynes derived from secondary propargyl alcohols, the crude reaction mixture was first filtered through a plug of silica to remove paramagnetic cobalt. Diastereomeric ratios were then determined by ${ }^{1} H$ NMR analysis. The mixture of diastereomers was further purified to obtain the clean spectra and yields of the major (syn) PKR products. 


\section{B. Relative Stereochemistry Determination}

The diagnostic ${ }^{1} \mathrm{H}$ NMR signals to differentiate the diastereomeric major (syn) vs. minor (anti) PKR products are the $\delta \mathrm{H}_{\mathrm{a}}$ and $\mathrm{H}_{\mathrm{b}}$ in both compounds: For example, $\delta \mathrm{H}_{\mathrm{a}}$ in $\mathbf{6 h}$ major (syn) is 5.23 ppm and $\delta \mathrm{H}_{\mathrm{a}}$ in $\mathbf{6 h}$ minor (anti) is $5.10 \mathrm{ppm} . \delta \mathrm{H}_{\mathrm{b}}$ in $\mathbf{6 h}$ major (syn) is $3.26 \mathrm{ppm}$ and $\delta \mathrm{H}_{\mathrm{b}}$ in $\mathbf{6 h}$ minor (anti) is $3.17 \mathrm{ppm}$. The chemical shift difference between the two sets of diastereomeric protons $\delta \mathrm{H}_{\mathrm{a}}$ and $\mathrm{H}_{\mathrm{b}}$ is consistent with a broad range of PKR products. The relative stereochemistry assigned is further corroborated through single X-ray diffraction studies of $6 \mathbf{e}, \mathbf{6 g}, 7 \mathbf{c}$, and 7e.
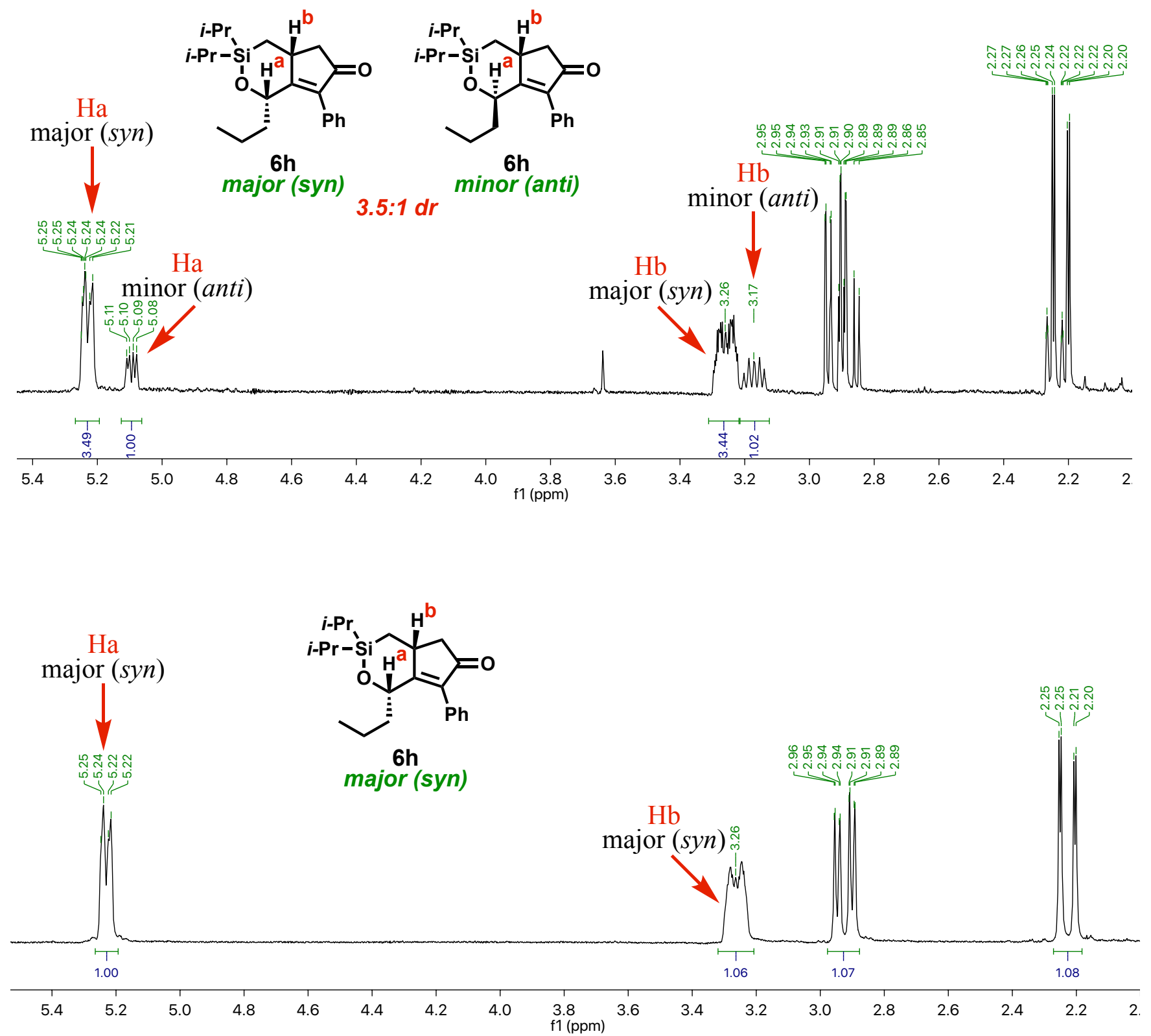


\section{3,3-Diisopropyl-7-methyl-3,4,4a,5-tetrahydrocyclopenta $[d][1,2]$ oxasilin-6(1H)-one (2a)}

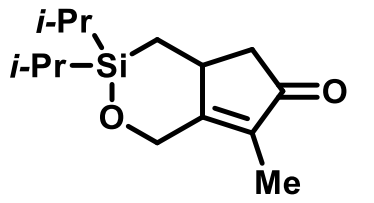

81\% yield (68.2 mg, $0.27 \mathrm{mmol})$; colorless oil. 4-FBnSMe S21 (216 mg,

$83 \%$ recovery).

$\mathbf{R}_{f}: 0.4$ (silica, 10\% EtOAc/hexanes).

${ }^{1}$ H NMR (400 MHz, $\mathbf{C D C l}_{3}$ ): $\delta 4.93$ (d, $\left.J=17.4 \mathrm{~Hz}, 1 \mathrm{H}\right), 4.77$ (d, $\left.J=17.6 \mathrm{~Hz}, 1 \mathrm{H}\right), 3.06$ (dddq, $J=13.5,7.3,3.7,1.8 \mathrm{~Hz}, 1 \mathrm{H}), 2.76(\mathrm{ddd}, J=18.8,6.2,1.0 \mathrm{~Hz}, 1 \mathrm{H}), 2.07$ (dt, $J=18.9,0.9 \mathrm{~Hz}$, 1H), $1.66-1.62(\mathrm{~m}, 3 \mathrm{H}), 1.30$ (dd, $J=14.5,5.3 \mathrm{~Hz}, 1 \mathrm{H}), 1.09$ (tdd, $J=4.8,3.4,2.0 \mathrm{~Hz}, 7 \mathrm{H}), 0.98$ (ddt, $J=11.2,4.3,1.9 \mathrm{~Hz}, 7 \mathrm{H}), 0.54$ (dd, $J=14.5,13.5 \mathrm{~Hz}, 1 \mathrm{H})$.

${ }^{13}$ C NMR (101 MHz, $\left.\mathbf{C D C l}_{3}\right): \delta 208.09,172.78,133.49,63.38,45.50,35.59,17.38,17.19,17.12$, $16.98,14.91,13.04,12.70,7.61$.

IR (neat, $\mathbf{c m}^{-1}$ ): 2940, 2921, 2892, 2865, 1702, 1649, 1463, 1409, 1382, 1329, 1292, 1205, 1163 , 1120, 1048, 995, 880, 800, 759, 727.

HRMS $\left(\mathbf{C I}^{+}, \boldsymbol{m} / \boldsymbol{z}\right)$ : calculated for $\mathrm{C}_{14} \mathrm{H}_{25} \mathrm{O}_{2} \mathrm{Si}[\mathrm{M}+\mathrm{H}]^{+}$253.1612483, found 253.16197.

\section{3,3-Diisopropyl-3,4,4a,5-tetrahydrocyclopenta[d][1,2]oxasilin-6(1H)-one $(2 \mathrm{~b})$}

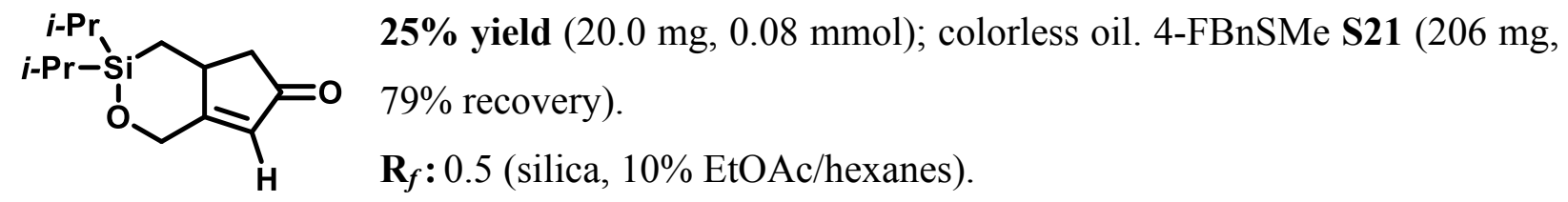

${ }^{1}$ H NMR (400 MHz, CDCl $): \delta 5.90(\mathrm{dq}, J=1.7,0.9 \mathrm{~Hz}, 1 \mathrm{H}), 4.91(\mathrm{dt}, J=16.9,1.7 \mathrm{~Hz}, 1 \mathrm{H})$, $4.82(\mathrm{dt}, J=16.8,1.6 \mathrm{~Hz}, 1 \mathrm{H}), 3.16(\mathrm{dtt}, J=13.5,5.8,1.6 \mathrm{~Hz}, 1 \mathrm{H}), 2.78(\mathrm{ddd}, J=18.7,6.3,0.8$ $\mathrm{Hz}, 1 \mathrm{H}), 2.14-2.06(\mathrm{~m}, 1 \mathrm{H}), 1.33(\mathrm{dd}, J=14.5,5.6 \mathrm{~Hz}, 1 \mathrm{H}), 1.13-1.06(\mathrm{~m}, 7 \mathrm{H}), 1.02-0.94(\mathrm{~m}$, $7 \mathrm{H}), 0.61(\mathrm{dd}, J=14.5,13.5 \mathrm{~Hz}, 1 \mathrm{H})$

${ }^{13}$ C NMR (101 MHz, $\left.\mathbf{C D C l}_{3}\right): \delta 208.21,181.53,126.90,64.40,46.54,37.55,17.46,17.29,17.12$, $17.01,15.17,12.87,12.78$.

IR (neat, $\mathbf{c m}^{-1}$ ): 2924, 2865, 1714, 1619, 1463, 1409, 1362, 1232, 1175, 1150, 1084, 1002, 917, $881,771,752,719$.

HRMS $\left(\mathbf{C I}^{+}, \boldsymbol{m} / \boldsymbol{z}\right)$ : calculated for $\mathrm{C}_{13} \mathrm{H}_{23} \mathrm{O}_{2} \mathrm{Si}[\mathrm{M}+\mathrm{H}]^{+} 239.14618$, found 239.14634. 


\section{3,3-Diisopropyl-7-(trimethylsilyl)-3,4,4a,5-tetrahydrocyclopenta[ $[d][1,2]$ oxasilin-6(1H)-one}

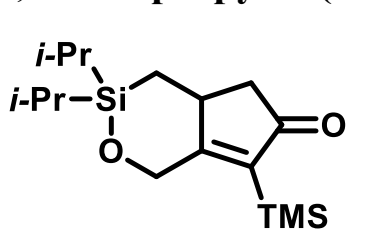

(2c)

43\% yield (44.7 mg, $0.14 \mathrm{mmol}$ ); colorless oil. 4-FBnSMe S21 (209 mg, $80 \%$ recovery).

$\mathbf{R}_{f}: 0.45$ (silica, 10\% EtOAc/hexanes).

${ }^{1}$ H NMR (400 MHz, CDCl 3 ): $\delta 5.01(\mathrm{dt}, J=17.8,1.2 \mathrm{~Hz}, 1 \mathrm{H}), 4.87(\mathrm{dt}, J=17.9,1.2 \mathrm{~Hz}, 1 \mathrm{H})$, $3.12(\mathrm{dddd}, J=11.2,5.3,4.2,3.2,1.5 \mathrm{~Hz}, 1 \mathrm{H}), 2.70(\mathrm{ddd}, J=18.5,6.6,1.0 \mathrm{~Hz}, 1 \mathrm{H}), 2.04(\mathrm{dd}, J=$ $18.3,2.1 \mathrm{~Hz}, 1 \mathrm{H}), 1.30(\mathrm{dd}, J=14.6,5.3 \mathrm{~Hz}, 1 \mathrm{H}), 1.11-1.05(\mathrm{~m}, 7 \mathrm{H}), 1.02-0.96(\mathrm{~m}, 7 \mathrm{H}), 0.57$ (dd, $J=14.7,13.6 \mathrm{~Hz}, 1 \mathrm{H}), 0.19$ (s, 9H).

${ }^{13}$ C NMR (101 MHz, $\left.\mathbf{C D C l}_{3}\right): \delta 212.11,189.37,136.91,65.38,46.60,38.86,17.30,17.23,17.12$, $17.05,14.90,13.12,12.67,-0.55$.

IR (neat, $\mathbf{c m}^{-1}$ ): 2924, 2866, 1693, 1585, 1462, 1408, 1248, 1098, 936, 881, 838, 750.8, 726.

HRMS $\left(\mathbf{C I}^{+}, \boldsymbol{m} / \boldsymbol{z}\right)$ : calculated for $\mathrm{C}_{16} \mathrm{H}_{31} \mathrm{O}_{2} \mathrm{Si}_{2}[\mathrm{M}+\mathrm{H}]^{+}$310.179900, found 310.178437 .

\section{3,3,7-Triisopropyl-3,4,4a,5-tetrahydrocyclopenta[d][1,2] oxasilin-6(1H)-one (2d)}

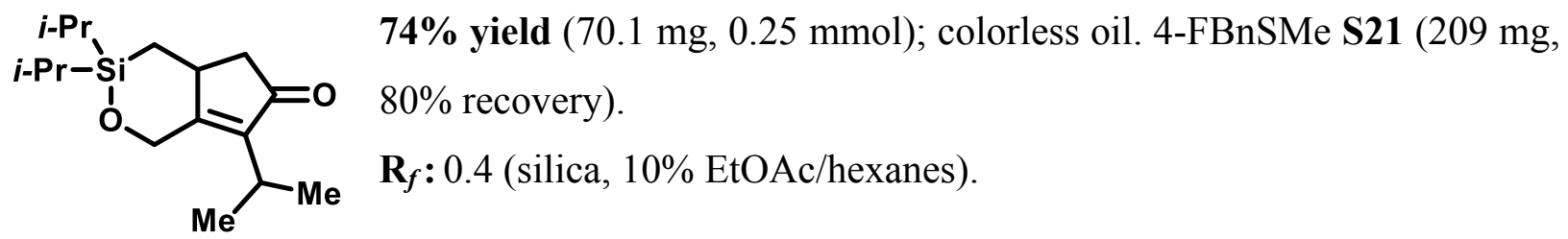

${ }^{1}$ H NMR (400 MHz, CDCl 3 ): $\delta 5.02(\mathrm{dt}, J=17.3,1.2 \mathrm{~Hz}, 1 \mathrm{H}), 4.81$ (dt, $\left.J=17.3,1.4 \mathrm{~Hz}, 1 \mathrm{H}\right)$, 3.01 (dddt, $J=13.3,6.9,5.3,1.7 \mathrm{~Hz}, 1 \mathrm{H}), 2.75-2.63(\mathrm{~m}, 2 \mathrm{H}), 2.03$ (dt, $J=18.8,0.8 \mathrm{~Hz}, 1 \mathrm{H})$, $1.29(\mathrm{dd}, J=14.6,5.2 \mathrm{~Hz}, 1 \mathrm{H}), 1.12(\mathrm{dd}, J=7.1,0.9 \mathrm{~Hz}, 6 \mathrm{H}), 1.10-1.06(\mathrm{~m}, 7 \mathrm{H}), 1.01-0.95$ (m, 7H), $0.52(\mathrm{dd}, J=14.6,13.5 \mathrm{~Hz}, 1 \mathrm{H})$.

${ }^{13}$ C NMR (101 MHz, $\left.\mathbf{C D C l}_{3}\right): \delta 207.78,172.28,142.31,63.36,45.95,35.24,24.50,20.50,20.11$, $17.31,17.16,17.11,17.01,15.02,13.06,12.67$.

IR (neat, $\mathbf{c m}^{-1}$ ): 2929, 2893, 2865, 1699, 1635, 1462, 1408, 1362, 1334, 1294, 1154, 1124, 1089, 992, 917, 881, 802, 761, 729.

HRMS ( $\left.\mathbf{C I}^{+}, \boldsymbol{m} / \boldsymbol{z}\right)$ : calculated for $\mathrm{C}_{16} \mathrm{H}_{29} \mathrm{O}_{2} \mathrm{Si}[\mathrm{M}+\mathrm{H}]^{+}$281.19313, found 281.19330. 


\section{3,3-Diisopropyl-7-((methoxymethoxy)methyl)-3,4,4a,5-}

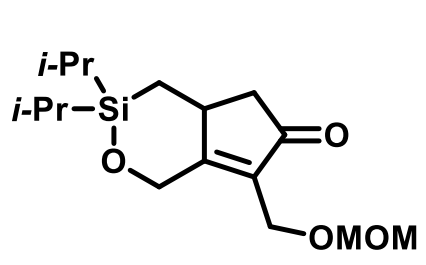
tetrahydrocyclopenta $[d][1,2]$ oxasilin-6(1H)-one $(2 \mathrm{e})$

67\% yield (68.8 mg, $0.22 \mathrm{mmol})$; colorless oil. 4-FBnSMe S21 (200 $\mathrm{mg}, 77 \%$ recovery).

$\mathbf{R}_{f}: 0.4$ (silica, 10\% EtOAc/hexanes).

${ }^{1}$ H NMR (400 MHz, CDCl $): \delta 5.10(\mathrm{~d}, J=18.0 \mathrm{~Hz}, 1 \mathrm{H}), 4.91(\mathrm{~d}, J=18.1 \mathrm{~Hz}, 1 \mathrm{H}), 4.59(\mathrm{~s}, 2 \mathrm{H})$, $4.19(\mathrm{~d}, J=11.8 \mathrm{~Hz}, 1 \mathrm{H}), 4.14(\mathrm{~d}, J=12.1 \mathrm{~Hz}, 1 \mathrm{H}), 3.34$ (s, 3H), 3.17 - 3.08 (m, 1H), 2.78 (ddd, $J=18.8,6.3,1.0 \mathrm{~Hz}, 1 \mathrm{H}), 2.09(\mathrm{dd}, J=18.7,1.9 \mathrm{~Hz}, 1 \mathrm{H}), 1.31(\mathrm{dd}, J=14.6,5.4 \mathrm{~Hz}, 1 \mathrm{H}), 1.11-$ $1.05(\mathrm{~m}, 7 \mathrm{H}), 1.01-0.94(\mathrm{~m}, 7 \mathrm{H}), 0.58(\mathrm{dd}, J=14.6,13.6 \mathrm{~Hz}, 1 \mathrm{H})$.

${ }^{13}$ C NMR (101 MHz, $\left.\mathbf{C D C l}_{3}\right): \delta 206.33,178.33,133.77,96.38,63.22,58.29,55.50,45.62,36.08$, $17.37,17.18,17.15,16.99,14.66,13.02,12.66$.

IR (neat, $\mathbf{c m}^{-1}$ ): 2927, 2893, 2865, 1702, 1648, 1463, 1409, 1291, 1210, 1151, 1103, 1039, 1021, $919,881,802,763,733$.

HRMS (CI $\left.{ }^{+}, \boldsymbol{m} / z\right)$ : calculated for $\mathrm{C}_{16} \mathrm{H}_{27} \mathrm{O}_{2} \mathrm{Si}[\mathrm{M}-\mathrm{H}]$ 311.16731, found 311.16775 .

3,3-Diisopropyl-7-phenyl-3,4,4a,5-tetrahydrocyclopenta $[d][1,2]$ oxasilin-6(1H)-one (2f)

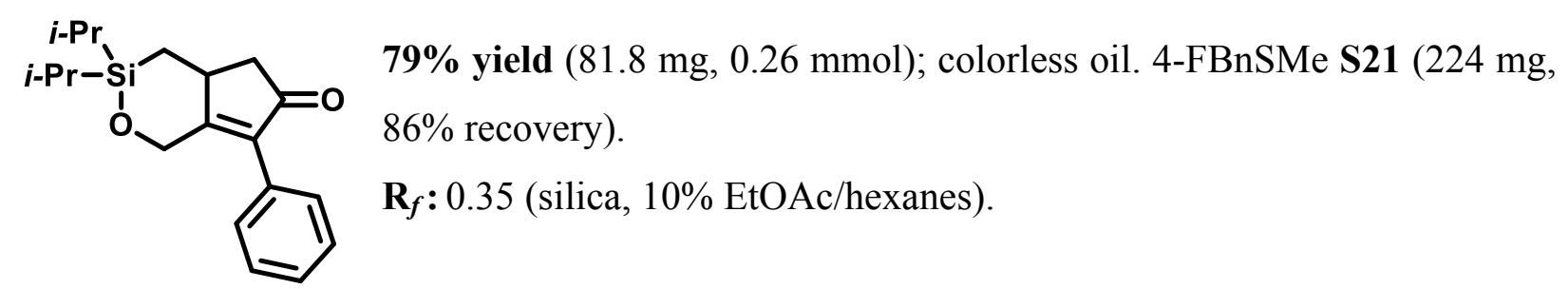

${ }^{1}$ H NMR (400 MHz, $\left.\mathbf{C D C l}_{3}\right): \delta 7.42-7.36(\mathrm{~m}, 2 \mathrm{H}), 7.35-7.30(\mathrm{~m}, 1 \mathrm{H}), 7.25-7.21(\mathrm{~m}, 2 \mathrm{H})$, $5.01(\mathrm{dt}, J=17.1,1.1 \mathrm{~Hz}, 1 \mathrm{H}), 4.93(\mathrm{dt}, J=17.1,1.7 \mathrm{~Hz}, 1 \mathrm{H}), 3.28-3.19$ (m, 1H), 2.93 (ddd, $J=$ 18.8, 6.4, $0.9 \mathrm{~Hz}, 1 \mathrm{H}), 2.27$ (dd, $J=18.9,1.9 \mathrm{~Hz}, 1 \mathrm{H}), 1.41(\mathrm{dd}, J=14.5,5.4 \mathrm{~Hz}, 1 \mathrm{H}), 1.25$ (s, $1 \mathrm{H}), 1.16-1.09(\mathrm{~m}, 7 \mathrm{H}), 1.00-0.95(\mathrm{~m}, 6 \mathrm{H}), 0.68(\mathrm{dd}, J=14.5,13.4 \mathrm{~Hz}, 1 \mathrm{H})$.

${ }^{13}$ C NMR (101 MHz, $\left.\mathbf{C D C l}_{3}\right): \delta$ 206.12, 174.43, 137.55, 130.88, 128.92, 128.45, 128.13, 63.78, $46.21,35.79,17.43,17.25,17.13,16.99,15.54,12.89,12.72$.

IR (neat, $\mathbf{c m}^{-1}$ ): 2924, 2864, 1695, 1623, 1463, 1294, 1092, 1009, 982, 884, 764, 706.

HRMS ( $\left.\mathbf{C I}^{+}, \boldsymbol{m} / \boldsymbol{z}\right)$ : calculated for $\mathrm{C}_{19} \mathrm{H}_{27} \mathrm{O}_{2} \mathrm{Si}[\mathrm{M}+\mathrm{H}]^{+} 315.17762$, found 315.17748. 


\section{3,3-Diisopropyl-7-(4-methoxyphenyl)-3,4,4a,5-tetrahydrocyclopenta[ $[\mathrm{d}][1,2]$ oxasilin-6(1H)- one (2g)}

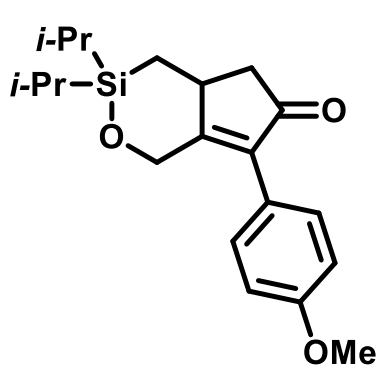

65\% yield (75.8 mg, $0.22 \mathrm{mmol}$ ); colorless oil. 4-FBnSMe S21 (201 mg, $77 \%$ recovery).

$\mathbf{R}_{f}: 0.3$ (silica, 10\% EtOAc/hexanes).

2H), $5.02(\mathrm{dt}, J=17.1,1.1 \mathrm{~Hz}, 1 \mathrm{H}), 4.93(\mathrm{dt}, J=17.0,1.7 \mathrm{~Hz}, 1 \mathrm{H}), 3.82(\mathrm{~s}, 3 \mathrm{H}), 3.26-3.17$ (m, 1H), 2.91 (ddd, $J=18.8,6.4,0.9 \mathrm{~Hz}, 1 \mathrm{H}), 2.25$ (dd, $J=18.7,1.9 \mathrm{~Hz}, 1 \mathrm{H}), 1.40$ (dd, $J=14.5,5.4$ $\mathrm{Hz}, 1 \mathrm{H}), 1.15-1.09(\mathrm{~m}, 7 \mathrm{H}), 1.01-0.93$ (m, 7H), $0.66(\mathrm{dd}, J=14.5,13.4 \mathrm{~Hz}, 1 \mathrm{H})$.

${ }^{13}$ C NMR (101 MHz, $\left.\mathbf{C D C l}_{3}\right): \delta$ 206.56, 173.41, 159.48, 137.04, 130.25, 123.22, 114.02, 63.96, $55.42,46.27,35.70,17.48,17.30,17.18,17.05,15.63,12.96,12.79$.

IR (neat, $\mathbf{c m}^{-1}$ ): 2925, 2864, 1699, 1606, 1510, 1462. 1289, 1247, 1177, 1095, 1032, 988, 915, $881,827,783,761$,

HRMS (CI, $\boldsymbol{m} / \mathbf{z})$ : calculated for $\mathrm{C}_{20} \mathrm{H}_{29} \mathrm{O}_{3} \mathrm{Si}[\mathrm{M}+\mathrm{H}]^{+}$344.180943, found 344.180773.

4-(3,3-Diisopropyl-6-oxo-1,3,4,4a,5,6-hexahydrocyclopenta[d][1,2] oxasilin-7-yl)benzonitrile<smiles>CC(C)[Si]1(C(F)(F)F)CC2CC(=O)C(c3ccc(C#N)cc3)=C2CO1</smiles>
(2h)

72\% yield (81.5 mg, $0.24 \mathrm{mmol})$; colorless oil. 4-FBnSMe S21 (214 mg, $82 \%$ recovery).

$\mathbf{R}_{f}: 0.3$ (silica, $10 \%$ EtOAc/hexanes).

${ }^{1}$ H NMR (400 MHz, $\left.\mathbf{C D C l}_{3}\right): \delta 7.68(\mathrm{~d}, J=8.1 \mathrm{~Hz}, 2 \mathrm{H}), 7.35(\mathrm{~d}, J=8.1 \mathrm{~Hz}, 2 \mathrm{H}), 4.94(\mathrm{dd}, J=$ 20.1, 3.5 Hz, 2H), $3.26(\mathrm{dt}, J=13.1,5.7 \mathrm{~Hz}, 1 \mathrm{H}), 2.94(\mathrm{dd}, J=19.0,6.4 \mathrm{~Hz}, 1 \mathrm{H}), 2.28(\mathrm{dd}, J=$ $18.9,1.9 \mathrm{~Hz}, 1 \mathrm{H}), 1.42(\mathrm{dd}, J=14.6,5.4 \mathrm{~Hz}, 1 \mathrm{H}), 1.14-1.07(\mathrm{~m}, 7 \mathrm{H}), 1.0-0.92(\mathrm{~m}, 7 \mathrm{H}), 0.68$ (t, $J=14.0 \mathrm{~Hz}, 1 \mathrm{H})$.

${ }^{13}$ C NMR (101 MHz, CDCl 3 ): $\delta$ 205.14, 176.75, 135.86, 135.70, 132.21, 129.65, 118.74, 111.82, 63.55, 46.06, 36.18, 29.80, 17.37, 17.19, 17.10, 16.96, 15.41, 12.84, 12.64 .

IR (neat, $\mathbf{c m}^{-1}$ ): 2941, 2864, 2227, 1701, 1605, 1503, 1462, 1406, 1336, 1293, 1098, 989, 915, $881,829,759,735,652,600,551,527,457$.

HRMS $\left(\mathbf{C I}^{+}, \boldsymbol{m} / \boldsymbol{z}\right)$ : calculated for $\mathrm{C}_{20} \mathrm{H}_{26} \mathrm{NO}_{2} \mathrm{Si}[\mathrm{M}+\mathrm{H}]^{+}$339.165700, found 339.165458. 


\section{Methyl-4-(3,3-diisopropyl-6-oxo-1,3,4,4a,5,6-hexahydrocyclopenta[d][1,2]oxasilin-7-}

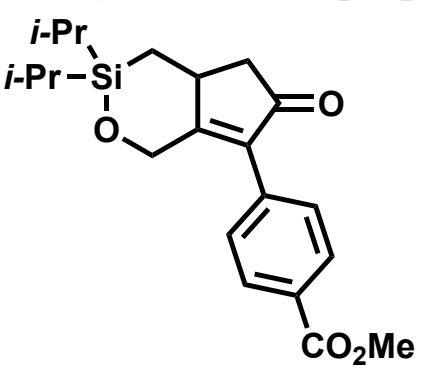
yl)benzoate (2i)

76\% yield (93.1 mg, $0.25 \mathrm{mmol}$ ); colorless oil. 4-FBnSMe S21 (206 $\mathrm{mg}, 79 \%$ recovery).

$\mathbf{R}_{f}: 0.25$ (silica, 10\% EtOAc/hexanes).

${ }^{1}$ H NMR (400 MHz, $\left.\mathbf{C D C l}_{3}\right): \delta 8.08$ - $8.03(\mathrm{~m}, 2 \mathrm{H}), 7.33-7.29$ (m, 2H), 4.98 (dt, $J=17.3,1.1$ $\mathrm{Hz}, 1 \mathrm{H}), 4.94$ (dt, $J=17.3,1.6 \mathrm{~Hz}, 1 \mathrm{H}), 3.91$ (s, 3H), $3.29-3.21$ (m, 1H), 2.94 (ddd, $J=18.9,6.4$, $0.8 \mathrm{~Hz}, 1 \mathrm{H}), 2.28(\mathrm{dd}, J=18.8,2.0 \mathrm{~Hz}, 1 \mathrm{H}), 2.06 \mathrm{dd}, J=15.7,6.3 \mathrm{~Hz}, 1 \mathrm{H}), 1.41(\mathrm{dd}, J=14.6,5.4$ $\mathrm{Hz}, 1 \mathrm{H}), 1.14-1.08(\mathrm{~m}, 7 \mathrm{H}), 1.0-0.93(\mathrm{~m}, 6 \mathrm{H}), 0.68(\mathrm{dd}, J=14.6,13.4 \mathrm{~Hz}, 1 \mathrm{H})$, .

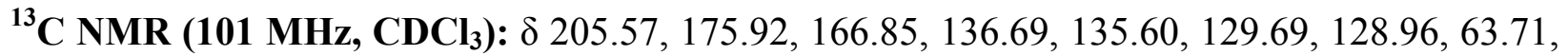
$52.30,46.17,36.05,17.40,17.23,17.12,16.99,15.46,12.89,12.68$.

IR (neat, $\mathbf{c m}^{-1}$ ): 2943, 2864, 1720, 1702, 1607, 1462, 1435, 1406, 1334, 1273, 1184, 1099, 989, $916,881,780,771,704,649,591$.

HRMS (CI, $\boldsymbol{m} / \boldsymbol{z})$ : calculated for $\mathrm{C}_{21} \mathrm{H}_{29} \mathrm{O}_{4} \mathrm{Si}[\mathrm{M}+\mathrm{H}]^{+}$372.176548, found 372.175688.

\section{3,3-Diisopropyl-7-(4-(trifluoromethyl)phenyl)-3,4,4a,5-}

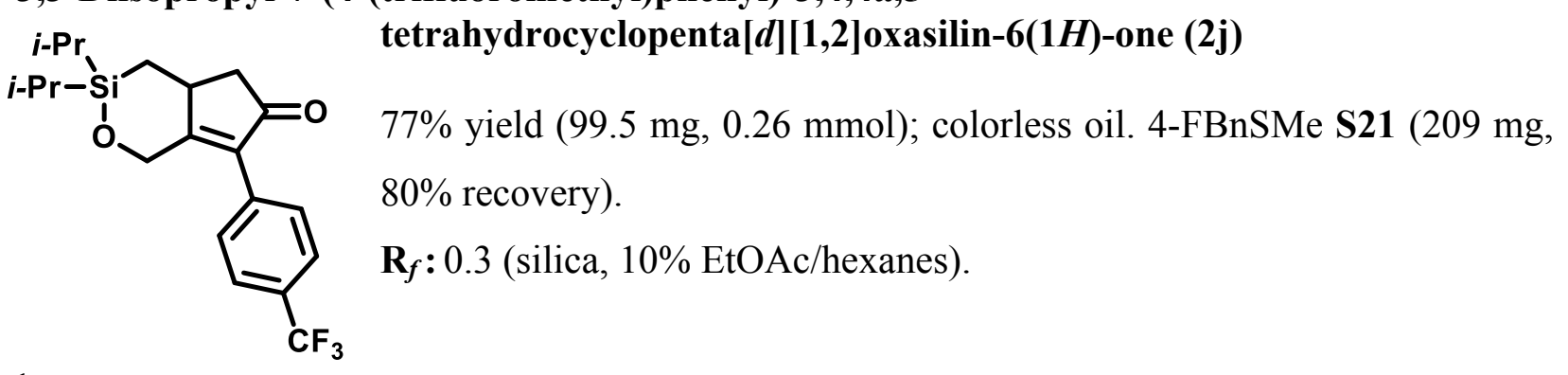

${ }^{1}$ H NMR (400 MHz, CDCl 3 ): $\delta 7.65(\mathrm{~d}, J=8.1 \mathrm{~Hz}, 2 \mathrm{H}), 7.35(\mathrm{~d}, J=8.0 \mathrm{~Hz}, 2 \mathrm{H}), 4.97$ (dt, $J=$ 17.3, 1.6 Hz, 1H), $4.94(\mathrm{dd}, J=17.3,1.6 \mathrm{~Hz}, 1 \mathrm{H}), 3.31-3.22(\mathrm{~m}, 1 \mathrm{H}), 2.94(\mathrm{dd}, J=18.9,6.4 \mathrm{~Hz}$, 1H), 2.28 (dd, $J=18.9,1.9 \mathrm{~Hz}, 1 \mathrm{H}), 1.43(\mathrm{dd}, J=14.6,5.5 \mathrm{~Hz}, 1 \mathrm{H}), 1.14-1.08$ (m, 7H), $1.00-$ $0.94(\mathrm{~m}, 7 \mathrm{H}), 0.69$ (dd, $J=14.6,13.4 \mathrm{~Hz}, 1 \mathrm{H})$.

${ }^{13}$ C NMR (101 MHz, CDCl $)$ ): $\delta$ 205.55, 176.10, 136.36, 134.62, 134.60, 130.64, 130.32, 130.00, 129.67, 129.31, 125.49, 125.46, 125.42, 125.38, 122.79, 63.62, 46.12, 36.09, 29.83, 17.40, 17.21, $17.11,16.98,15.46,12.89,12.70 .\left({ }^{1} J_{\mathrm{C}-\mathrm{F}}=130.64,130.32,130.00,129.67\right)$.

IR (neat, $\mathbf{c m}^{-1}$ ): 2943, 2866, 1702, 1615, 1463, 1408, 1322, 1293, 1165, 1120, 1065, 1019, 989, $880,831,813,760,745,728,683,649,604$.

HRMS (CI $\left.{ }^{+}, \boldsymbol{m} / \boldsymbol{z}\right)$ : calculated for $\mathrm{C}_{20} \mathrm{H}_{26} \mathrm{~F}_{3} \mathrm{O}_{2} \mathrm{Si}[\mathrm{M}+\mathrm{H}]^{+}$382.156432, found 382.157593. 
(1S,4aR)-1-isobutyl-3,3-diisopropyl-7-methyl-3,4,4a,5-tetrahydrocyclopenta[d][1,2]oxasilin6(1H)-one (6a)

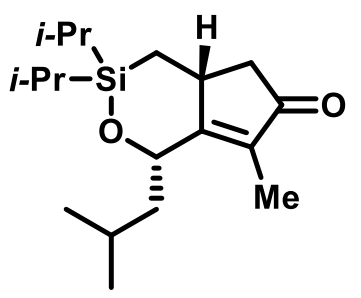

6:1 dr, 31\% yield (31.1 mg, $0.101 \mathrm{mmol}$ ); white solid. 4-FBnSMe S21 (200 mg, 77\% recovery).

$\mathbf{R}_{f}: 0.4$ (silica, 10\% EtOAc/hexanes).

${ }^{1}$ H NMR (400 MHz, $\left.\mathbf{C D C l}_{3}\right): \delta 5.01(\mathrm{dd}, J=10.1,3.3 \mathrm{~Hz}, 1 \mathrm{H}), 3.06-2.96(\mathrm{~m}, 1 \mathrm{H}), 2.70(\mathrm{dd}, J$ $=18.8,6.2 \mathrm{~Hz}, 1 \mathrm{H}), 2.06(\mathrm{~d}, J=18.8 \mathrm{~Hz}, 1 \mathrm{H}), 2.00-1.86(\mathrm{~m}, 1 \mathrm{H}), 1.71-1.56(\mathrm{~m}, 4 \mathrm{H}), 1.31-$ $1.10(\mathrm{~m}, 3 \mathrm{H}), 1.08-1.00(\mathrm{~m}, 7 \mathrm{H}), 0.99-0.88(\mathrm{~m}, 12 \mathrm{H}), 0.44(\mathrm{dd}, J=14.5,13.2 \mathrm{~Hz}, 1 \mathrm{H})$.

${ }^{13}$ C NMR (101 MHz, $\left.\mathbf{C D C l}_{3}\right): \delta 208.98,177.70,132.92,70.53,45.92,45.67,34.03,24.41,23.74$, $21.57,17.24,17.14,17.05,17.02,16.10,13.40,13.05,8.02$.

IR (neat, $\mathbf{c m}^{-1}$ ): 2925, 2866, 1703, 1647, 1464, 1383, 991, 880, 759.

HRMS (CI $\left.{ }^{+}, \boldsymbol{m} / \boldsymbol{z}\right)$ : calculated for $\mathrm{C}_{18} \mathrm{H}_{33} \mathrm{O}_{2} \mathrm{Si}[\mathrm{M}+\mathrm{H}]^{+}$309.224984, found 309.226161.

(1S,4aR)-3,3-diisopropyl-7-methyl-1-propyl-3,4,4a,5-tetrahydrocyclopenta[d][1,2]oxasilin6(1H)-one (6b)

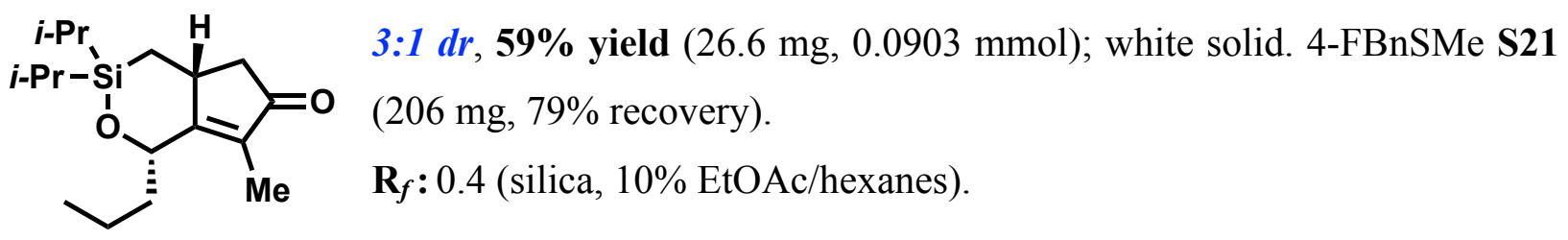

${ }^{1}$ H NMR (400 MHz, $\left.\mathbf{C D C l}_{3}\right): \delta 4.97(\mathrm{dd}, J=8.4,3.4 \mathrm{~Hz}, 1 \mathrm{H}), 3.01$ (dtd, $J=13.3,5.8,1.7 \mathrm{~Hz}$, 1H), 2.72 (dd, $J=18.8,6.1 \mathrm{~Hz}, 1 \mathrm{H}), 2.07$ (dd, $J=18.9,1.1 \mathrm{~Hz}, 1 \mathrm{H}), 1.66(\mathrm{~d}, J=1.8 \mathrm{~Hz}, 3 \mathrm{H}), 1.55$ $-1.39(\mathrm{~m}, 2 \mathrm{H}), 1.28(\mathrm{dd}, J=14.5,5.7 \mathrm{~Hz}, 1 \mathrm{H}), 1.10-1.00(\mathrm{~m}, 8 \mathrm{H}), 0.98-0.89(\mathrm{~m}, 10 \mathrm{H}), 0.45$ (dd, $J=14.5,13.2 \mathrm{~Hz}, 1 \mathrm{H})$.

${ }^{13}$ C NMR (101 MHz, $\left.\mathbf{C D C l}_{3}\right): \delta 209.00,177.23,133.33,72.03,45.62,39.28,34.28,18.54,17.20$, $17.07,17.01,16.98,16.08,14.01,13.36,13.03,8.14$.

IR (neat, $\mathbf{c m}^{-1}$ ): 2925, 2867, 1703, 1644, 1463, 1411, 1386, 1335, 1297, 1170, 1140, 1106, 1068, $1036,985,881,805,779,690$.

HRMS (CI $\left.{ }^{+}, \boldsymbol{m} / \boldsymbol{z}\right)$ : calculated for $\mathrm{C}_{17} \mathrm{H}_{31} \mathrm{O}_{2} \mathrm{Si}[\mathrm{M}+\mathrm{H}]^{+}$295.20878, found 295.20884. 
(1S,4aR)-1-isobutyl-3,3-diisopropyl-7-phenyl-3,4,4a,5-tetrahydrocyclopenta[d][1,2]oxasilin6(1H)-one (6c)

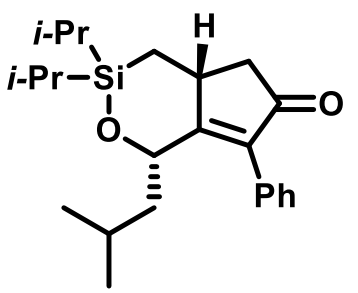

5.5:1 dr, 81\% yield (100.4 mg, 0.271 mmol); white solid. 4-FBnSMe S21 (219 $\mathrm{mg}, 84 \%$ recovery).

$\mathbf{R}_{f}: 0.4$ (silica, 10\% EtOAc/hexanes).

${ }^{1}$ H NMR (400 MHz, CDCl $)$ ): $\delta 7.39$ - $7.33(\mathrm{~m}, 2 \mathrm{H}), 7.32$ - $7.28(\mathrm{~m}, 1 \mathrm{H}), 7.19-7.15$ (m, 2H), $5.31(\mathrm{~d}, J=11.3 \mathrm{~Hz}, 1 \mathrm{H}), 3.31-3.22(\mathrm{~m}, 1 \mathrm{H}), 2.92(\mathrm{ddd}, J=18.6,6.3,1.2 \mathrm{~Hz}, 1 \mathrm{H}), 2.22(\mathrm{dd}, J=$ $18.5,2.9 \mathrm{~Hz}, 1 \mathrm{H}), 1.87-1.74(\mathrm{~m}, 1 \mathrm{H}), 1.38-1.28(\mathrm{~m}, 2 \mathrm{H}), 1.17-1.10(\mathrm{~m}, 7 \mathrm{H}), 1.09-0.98(\mathrm{~m}$, $7 \mathrm{H}), 0.98-0.82(\mathrm{~m}, 2 \mathrm{H}), 0.71-0.63(\mathrm{~m}, 3 \mathrm{H}), 0.58(\mathrm{~d}, J=6.5 \mathrm{~Hz}, 3 \mathrm{H})$.

${ }^{13}$ C NMR (101 MHz, $\left.\mathbf{C D C l}_{3}\right): \delta$ 205.85, 180.36, 137.96, 132.27, 128.96, 128.53, 127.96, 72.15, $46.08,46.07,36.79,23.90,23.44,20.19,18.17,17.84,17.27,17.15,15.58,13.82,12.67$.

IR (neat, $\mathbf{c m}^{-1}$ ): 2953, 2866, 1702, 1466, 1143, 1131, 1085, 1017, 881, 755, 697.

HRMS $\left(\mathbf{C I}^{+}, \boldsymbol{m} / \boldsymbol{z}\right)$ : calculated for $\mathrm{C}_{23} \mathrm{H}_{35} \mathrm{O}_{2} \mathrm{Si}[\mathrm{M}+\mathrm{H}]^{+} 371.24008$, found 371.24017.

(1S,4aR)-1-benzyl-3,3-diisopropyl-7-phenyl-3,4,4a,5-tetrahydrocyclopenta[d][1,2]oxasilin-

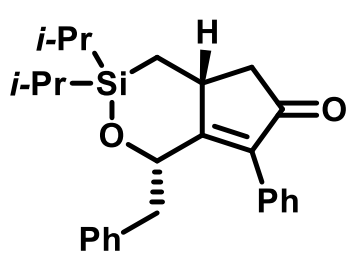
6(1H)-one (6e)

13:1 dr, 88\% yield (1.07 g, $2.64 \mathrm{mmol})$; white solid. 4-FBnSMe S21 (1.85 g, $89 \%$ recovery).

$\mathbf{R}_{f}: 0.4$ (silica, 10\% EtOAc/hexanes).

MP: $121-125^{\circ} \mathrm{C}$.

${ }^{1}$ H NMR (400 MHz, CDCl $): \delta 7.44-7.38(\mathrm{~m}, 2 \mathrm{H}), 7.35-7.30(\mathrm{~m}, 1 \mathrm{H}), 7.25-7.11(\mathrm{~m}, 5 \mathrm{H})$, $6.98-6.93(\mathrm{~m}, 2 \mathrm{H}), 5.49(\mathrm{dt}, J=10.4,1.8 \mathrm{~Hz}, 1 \mathrm{H}), 3.29(\mathrm{ddt}, J=13.8,6.5,2.6 \mathrm{~Hz}, 1 \mathrm{H}), 2.97$ (ddd, $J=18.7,6.4,1.0 \mathrm{~Hz}, 1 \mathrm{H}), 2.74(\mathrm{dd}, J=14.7,2.5 \mathrm{~Hz}, 1 \mathrm{H}), 2.58$ (dd, $J=14.7,10.5 \mathrm{~Hz}, 1 \mathrm{H})$, $2.29(\mathrm{dd}, J=18.6,2.7 \mathrm{~Hz}, 1 \mathrm{H}), 1.41(\mathrm{dd}, J=14.6,4.5 \mathrm{~Hz}, 1 \mathrm{H}), 1.16-0.93(\mathrm{~m}, 14 \mathrm{H}), 0.75(\mathrm{t}, J=$ $14.4 \mathrm{~Hz}, 1 \mathrm{H})$.

${ }^{13}$ C NMR (101 MHz, $\left.\mathbf{C D C l}_{3}\right): \delta$ 206.23, 177.83, 138.81, 138.78, 132.34, 129.09, 128.93, 128.77, $128.28,128.16,126.26,75.10,46.20,42.84,37.22$, 18.03, 17.72, 17.17, 17.15, 15.15, 14.67, 12.52 . IR (neat, $\mathbf{c m}^{-1}$ ): 2939, 2863, 1594, 1497, 1455, 1686, 1096, 1054, 923, 883, 723, 758, 697. HRMS ( $\left.\mathbf{C I}^{+}, \boldsymbol{m} / \boldsymbol{z}\right)$ : calculated for $\mathrm{C}_{26} \mathrm{H}_{33} \mathrm{O}_{2} \mathrm{Si}[\mathrm{M}+\mathrm{H}]^{+}$405.22443, found 405.22429. 


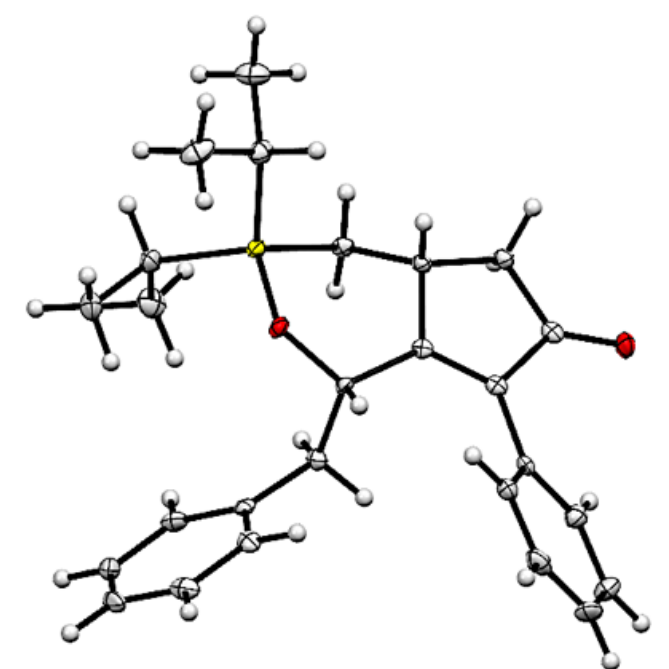

Table S1. Crystal data and structure refinement for (6e).

CCDC Number

Empirical formula

Formula weight

Temperature/K

Crystal system

Space group

a

b

C

$\beta$

Volume

Z

$\mathrm{d}_{\text {calc }}$

$\mu$

$\mathrm{F}(000)$

Crystal size, $\mathrm{mm}$

$2 \theta$ range for data collection

Index ranges

Reflections collected

Independent reflections

Data/restraints/parameters

Goodness-of-fit on $\mathrm{F}^{2}$

Final $R$ indexes $[l>=2 \sigma(I)]$

Final $R$ indexes [all data]

Largest diff. peak/hole
1944609

$\mathrm{C}_{26} \mathrm{H}_{32} \mathrm{O}_{2} \mathrm{Si}$

404.60

100

monoclinic

$\mathrm{P} 2{ }_{1} / \mathrm{C}$

$6.9808(7) \AA$

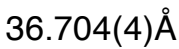

$17.5047(16) \AA$

$93.658(3)^{\circ}$

$4475.9(7) \AA^{3}$

8

$1.201 \mathrm{~g} / \mathrm{cm}^{3}$

$0.124 \mathrm{~mm}^{-1}$

1744.0

$0.43 \times 0.05 \times 0.02$

$5.848-50.792^{\circ}$

$-8 \leq h \leq 8,-44 \leq k \leq 44,-21 \leq l \leq 21$

70606

$8214[R$ (int) $=0.0587]$

$8214 / 0 / 531$

1.079

$\mathrm{R}_{1}=0.0487, \mathrm{wR}_{2}=0.0951$

$\mathrm{R}_{1}=0.0654, \mathrm{wR}_{2}=0.1012$

$0.35 /-0.32 \mathrm{e}^{-3}$ 
(1S,4aR)-3,3-diisopropyl-1-phenethyl-7-phenyl-3,4,4a,5-

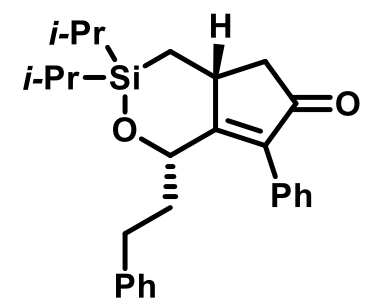

tetrahydrocyclopenta[d][1,2]oxasilin-6(1H)-one (6f)

4:1 dr, 84\% yield (117.6 mg, $0.281 \mathrm{mmol}$ ); white solid. 4-FBnSMe S21 (209 $\mathrm{mg}, 77 \%$ recovery).

$\mathbf{R}_{f}: 0.4$ (silica, 10\% EtOAc/hexanes).

${ }^{1}$ H NMR (400 MHz, CDCl $)$ ): $\delta 7.36$ - $7.28(\mathrm{~m}, 3 \mathrm{H}), 7.23-7.11(\mathrm{~m}, 3 \mathrm{H}), 7.09-7.02$ (m, 2H), $6.85-6.79(\mathrm{~m}, 2 \mathrm{H}), 5.14(\mathrm{ddt}, J=10.8,2.5,1.3 \mathrm{~Hz}, 1 \mathrm{H}), 3.30-3.20(\mathrm{~m}, 1 \mathrm{H}), 2.92(\mathrm{ddd}, J=18.7$, 6.4, 1.2 Hz, 1H), 2.69 (ddd, $J=13.2,8.4,4.5 \mathrm{~Hz}, 1 \mathrm{H}), 2.58$ (dt, $J=13.7,8.3 \mathrm{~Hz}, 1 \mathrm{H}), 2.23$ (dd, $J$ $=18.6,2.8 \mathrm{~Hz}, 1 \mathrm{H}), 1.73-1.53(\mathrm{~m}, 2 \mathrm{H}), 1.36(\mathrm{dd}, J=14.7,4.3 \mathrm{~Hz}, 1 \mathrm{H}), 1.19-1.12(\mathrm{~m}, 9 \mathrm{H}), 1.08$ $-1.03(\mathrm{~m}, 3 \mathrm{H}), 1.03-0.86(\mathrm{~m}, 2 \mathrm{H}), 0.69(\mathrm{t}, J=14.5 \mathrm{~Hz}, 1 \mathrm{H})$.

${ }^{13}$ C NMR (101 MHz, CDCl 3 ): $\delta$ 207.47, 176.87, 136.10, 134.17, 131.07, 129.14, 128.38, 128.02, $113.82,64.75,41.78,36.11,35.92,35.62,19.50,19.19,17.54,17.47,17.41,12.72,12.68$.

IR (neat, $\mathbf{c m}^{-1}$ ): 3027, 2924, 2864, 1702, 1495, 1462, 1407, 1290, 1129, 1097, 1074, 1050, 989, 919, 881, 857, 805, 754, 696, 667.

HRMS ( $\left.\mathbf{C I}^{+}, \boldsymbol{m} / \boldsymbol{z}\right)$ : calculated for $\mathrm{C}_{27} \mathrm{H}_{35} \mathrm{O}_{2} \mathrm{Si}[\mathrm{M}+\mathrm{H}]^{+} 419.24008$, found 419.24024.

(1S,4aR)-1-allyl-3,3-diisopropyl-7-phenyl-3,4,4a,5-tetrahydrocyclopenta[d][1,2]oxasilin6(1H)-one (6g)

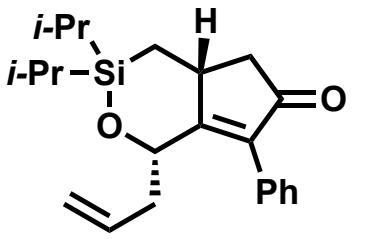

$>20: 1 d r, 87 \%$ yield (2.36 g, $6.65 \mathrm{mmol})$; white solid. 4-FBnSMe S21 (5.20 g, 87\% recovery).

$\mathbf{R}_{f}: 0.4$ (silica, $10 \%$ EtOAc/hexanes).

MP: $94-98{ }^{\circ} \mathrm{C}$.

${ }^{1}$ H NMR (400 MHz, $\left.\mathbf{C D C l}_{3}\right): \delta 7.43-7.28$ (m, 3H), 7.23 - 7.15 (m, 2H), 5.75 (ddt, $J=17.1$, $11.9,5.4 \mathrm{~Hz}, 1 \mathrm{H}), 5.30(\mathrm{~d}, J=10.0 \mathrm{~Hz}, 1 \mathrm{H}), 4.94$ (d, $J=10.3 \mathrm{~Hz}, 1 \mathrm{H}), 4.75$ (d, $J=17.1 \mathrm{~Hz}, 1 \mathrm{H})$, $3.31-3.22(\mathrm{~m}, 1 \mathrm{H}), 2.94(\mathrm{dd}, J=18.7,6.3 \mathrm{~Hz}, 1 \mathrm{H}), 2.25(\mathrm{dd}, J=18.7,2.7 \mathrm{~Hz}, 1 \mathrm{H}), 2.20-1.99$ $(\mathrm{m}, 2 \mathrm{H}), 1.37(\mathrm{dd}, J=14.7,4.3 \mathrm{~Hz}, 1 \mathrm{H}), 1.20-0.92(\mathrm{~m}, 14 \mathrm{H}), 0.68(\mathrm{t}, J=14.4 \mathrm{~Hz}, 1 \mathrm{H})$ ${ }^{13}$ C NMR (101 MHz, CDCl 3 ): $\delta$ 206.01, 177.89, 138.41, 135.02, 132.24, 128.83, 128.63, 128.13, $117.08,74.50,46.03,40.92,36.81,18.08,17.77,17.31,17.11,15.20,14.21,12.62$.

IR (neat, $\mathbf{c m}^{-1}$ ): 2937, 2863, 1696, 1463, 1406, 1292, 1141, 1123, 1082, 915, 882, 808, 758, 698, 669.

HRMS $\left(\mathbf{C I}^{+}, \boldsymbol{m} / \boldsymbol{z}\right)$ : calculated for $\mathrm{C}_{22} \mathrm{H}_{31} \mathrm{O}_{2} \mathrm{Si}[\mathrm{M}+\mathrm{H}]^{+}$355.20878, found 355.20860. 


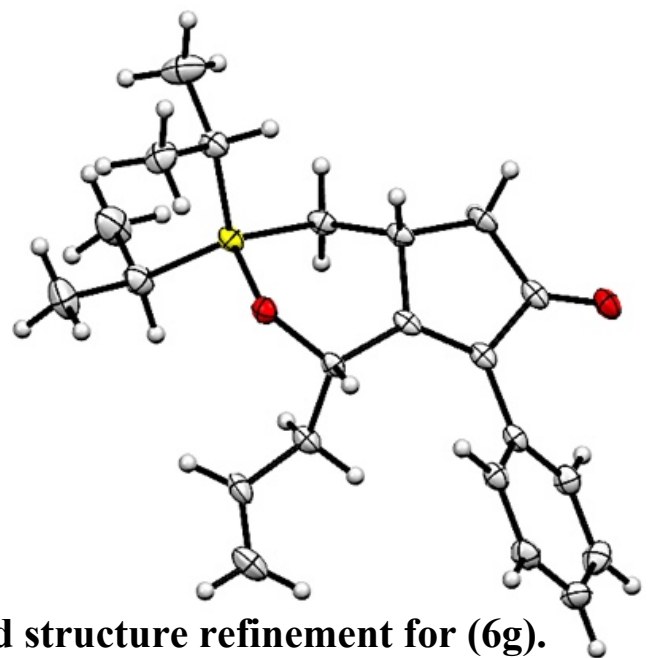

Table S2. Crystal data and structure refinement for (6g).

CCDC Number

Empirical formula

Formula weight

Temperature/K

Crystal system

Space group

a

b

c

$\alpha$

$\beta$

$\gamma$

Volume

Z

$\mathrm{d}_{\text {calc }}$

$\mu$

$\mathrm{F}(000)$

Crystal size, $\mathrm{mm}$

$2 \theta$ range for data collection

Index ranges

Reflections collected

Independent reflections

Data/restraints/parameters

Goodness-of-fit on $\mathrm{F}^{2}$

Final $\mathrm{R}$ indexes $[\mathrm{I}>=2 \sigma(\mathrm{I})]$

Final $\mathrm{R}$ indexes [all data]

Largest diff. peak/hole
1944610

$\mathrm{C}_{22} \mathrm{H}_{30} \mathrm{O}_{2} \mathrm{Si}$

354.55

100

triclinic

P1

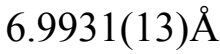

$11.8301(19) \AA$

$13.352(2) \AA$

$75.235(6)^{\circ}$

$77.460(6)^{\circ}$

$78.348(6)^{\circ}$

$1030.0(3) \AA^{3}$

2

$1.143 \mathrm{~g} / \mathrm{cm}^{3}$

$0.126 \mathrm{~mm}^{-1}$

384.0

$0.16 \times 0.08 \times 0.03$

$3.604-50.734^{\circ}$

$-8 \leq \mathrm{h} \leq 8,-13 \leq \mathrm{k} \leq 14,-16 \leq 1 \leq 16$

19352

$3700[\mathrm{R}($ int $)=0.0527]$

$3700 / 0 / 230$

1.029

$\mathrm{R}_{1}=0.0397, \mathrm{wR}_{2}=0.0874$

$\mathrm{R}_{1}=0.0593, \mathrm{wR}_{2}=0.0954$

$0.32 /-0.27$ e $\AA^{-3}$ 
(1S,4aR)-3,3-diisopropyl-7-phenyl-1-propyl-3,4,4a,5-tetrahydrocyclopenta[d][1,2]oxasilin6(1H)-one (6h)

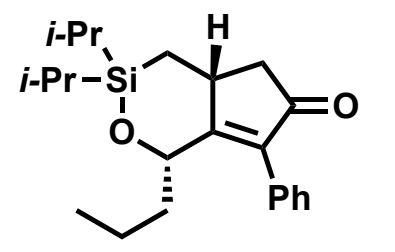

3.5:1 dr, 74\% yield (88.1 mg, $0.247 \mathrm{mmol}$ ); white solid. 4-FBnSMe S21 (214 $\mathrm{mg}, 82 \%$ recovery).

$\mathbf{R}_{f}: 0.4$ (silica, 10\% EtOAc/hexanes).

${ }^{1}$ H NMR (400 MHz, $\left.\mathbf{C D C l}_{3}\right): \delta 7.40$ - $7.34(\mathrm{~m}, 2 \mathrm{H}), 7.32-7.27(\mathrm{~m}, 1 \mathrm{H}), 7.20-7.16(\mathrm{~m}, 2 \mathrm{H})$, $5.23(\mathrm{dd}, J=9.4,3.4 \mathrm{~Hz}, 1 \mathrm{H}), 3.31-3.23$ (m, 1H), 2.92 (dd, $J=18.6,6.3 \mathrm{~Hz}, 1 \mathrm{H}), 2.23$ (dd, $J=$ $18.5,2.8 \mathrm{~Hz}, 1 \mathrm{H}), 1.47-1.22(\mathrm{~m}, 6 \mathrm{H}), 1.16-1.11(\mathrm{~m}, 7 \mathrm{H}), 1.08-0.98(\mathrm{~m}, 6 \mathrm{H}), 0.97-0.87(\mathrm{~m}$, $1 \mathrm{H}), 0.72-0.62(\mathrm{~m}, 3 \mathrm{H})$.

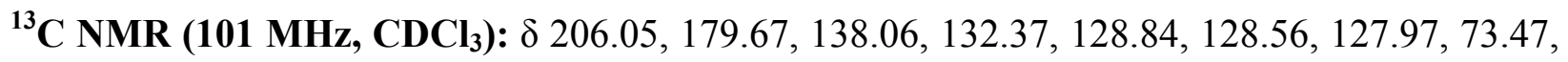
$46.05,38.87,36.83,18.98,18.13,17.81,17.34,17.17,15.28,13.93,13.11,12.70$.

IR (neat, $\mathbf{c m}^{-1}$ ): 2942, 2865, 1702, 1495, 1463, 1408, 1288, 1225, 1127, 1071, 1034, 1012, 919, $881,756,697,667$.

HRMS ( $\left.\mathbf{C I}^{+}, \boldsymbol{m} / \boldsymbol{z}\right)$ : calculated for $\mathrm{C}_{23} \mathrm{H}_{33} \mathrm{O}_{2} \mathrm{Si}[\mathrm{M}+\mathrm{H}]^{+}$357.22443, found 357.22439.

(1S,4aR)-3,3-diisopropyl-1-(pent-4-en-1-yl)-7-phenyl-3,4,4a,5-

tetrahydrocyclopenta[d][1,2]oxasilin-6(1H)-one (6i)

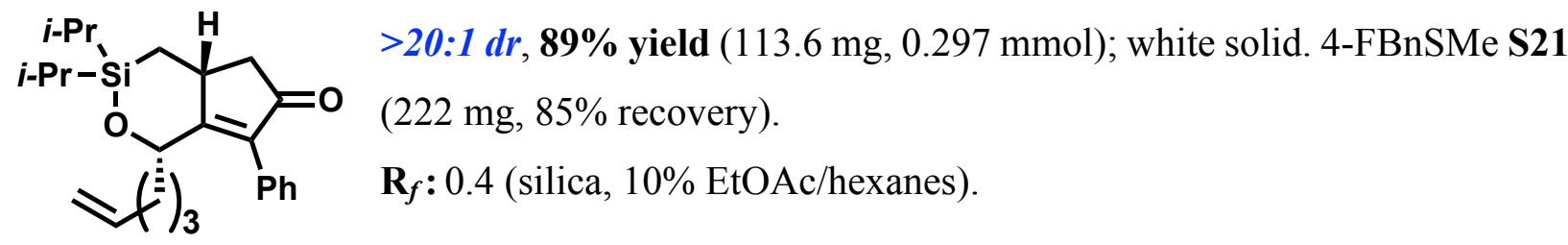

${ }^{1}$ H NMR (400 MHz, CDCl $)$ ): $\delta 7.43$ - 7.37 (m, 2H), 7.36 - 7.30 (m, 1H), $7.21-7.17$ (m, 2H), $5.62(\mathrm{ddt}, J=16.9,10.1,2.0 \mathrm{~Hz}, 1 \mathrm{H}), 5.06-5.03(\mathrm{~m}, 1 \mathrm{H}), 4.80-4.71(\mathrm{~m}, 2 \mathrm{H}), 3.24-3.16(\mathrm{~m}$, $1 \mathrm{H}), 2.72(\mathrm{dd}, J=19.1,6.7 \mathrm{~Hz}, 1 \mathrm{H}), 2.30-2.22(\mathrm{~m}, 1 \mathrm{H}), 2.14-2.00(\mathrm{~m}, 3 \mathrm{H}), 1.68-1.57(\mathrm{~m}$, 2H), $1.44(\mathrm{dt}, J=8.1,1.4 \mathrm{~Hz}, 2 \mathrm{H}), 1.27-1.12(\mathrm{~m}, 1 \mathrm{H}), 0.93-0.75(\mathrm{~m}, 14 \mathrm{H})$.

${ }^{13}$ C NMR (101 MHz, $\left.\mathbf{C D C l}_{3}\right): \delta$ 207.47, 176.87, 136.10, 134.17, 131.07, 129.14, 128.38, 128.02, $113.82,64.75,41.78,36.11,35.92,35.62,19.50,19.19,17.54,17.47,17.41,12.72,12.68$.

IR (neat, $\mathbf{c m}^{-1}$ ): 2938, 1865, 1705, 1630, 1463, 1449, 1291, 1138, 1114, 1072, 1026, 929, 882, $828,785,758,712,697$.

HRMS (CI $\left.{ }^{+}, \boldsymbol{m} / \boldsymbol{z}\right)$ : calculated for $\mathrm{C}_{24} \mathrm{H}_{35} \mathrm{O}_{2} \mathrm{Si}[\mathrm{M}+\mathrm{H}]^{+}$383.24008, found 383.24012. 
(1R,4aS)-3,3-diisopropyl-1-pentyl-7-phenyl-3,4,4a,5-tetrahydrocyclopenta[d][1,2]oxasilin6(1H)-one (6j)

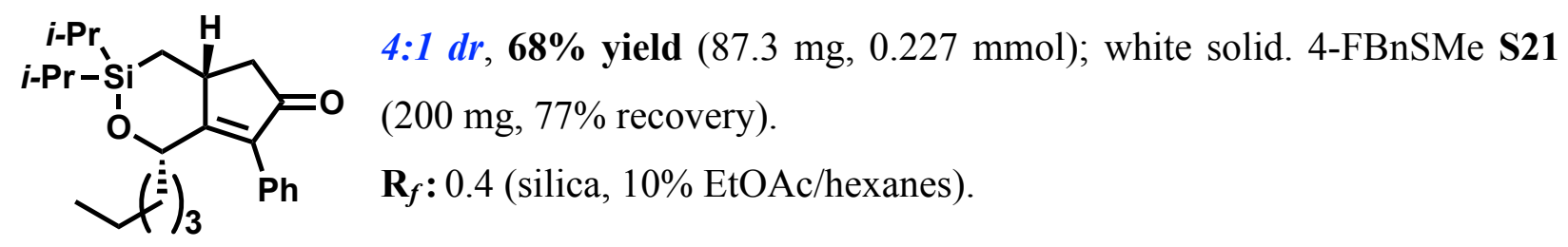

${ }^{1}$ H NMR (400 MHz, CDCl $): \delta 7.43-7.27(\mathrm{~m}, 3 \mathrm{H}), 7.20-7.13(\mathrm{~m}, 2 \mathrm{H}), 5.21$ (d, J=9.7 Hz, $1 \mathrm{H}), 3.31-3.21(\mathrm{~m}, 1 \mathrm{H}), 2.92(\mathrm{ddd}, J=18.5,6.3,1.2 \mathrm{~Hz}, 1 \mathrm{H}), 2.23(\mathrm{dd}, J=18.6,2.8 \mathrm{~Hz}, 1 \mathrm{H})$, $1.45-1.18(\mathrm{~m}, 7 \mathrm{H}), 1.16-0.98(\mathrm{~m}, 15 \mathrm{H}), 0.97-0.82(\mathrm{~m}, 2 \mathrm{H}), 0.75(\mathrm{t}, J=7.1 \mathrm{~Hz}, 2 \mathrm{H}), 0.67(\mathrm{t}, J$ $=14.5 \mathrm{~Hz}, 1 \mathrm{H})$.

${ }^{13}$ C NMR (101 MHz, $\left.\mathbf{C D C l}_{3}\right): \delta$ 206.05, 179.69, 132.42, 128.83, 128.60, 127.97, 73.99, 46.07, $36.84,36.79,30.82,25.54,22.32,18.16,17.83,17.37,17.17,15.34,14.03,13.96,12.71$.

IR (neat, $\mathbf{c m}^{-1}$ ): 2926, 2863, 1689, 1460, 1143, 1123, 1081, 1015, 920, 881, 859, 808, 765, 703.

HRMS $\left(\mathbf{C I}^{+}, \boldsymbol{m} / \boldsymbol{z}\right)$ : calculated for $\mathrm{C}_{24} \mathrm{H}_{37} \mathrm{O}_{2} \mathrm{Si}[\mathrm{M}+\mathrm{H}]^{+}$385.25573, found 385.25566.

(1S,4aR)-1-allyl-3,3-diisopropyl-7-methyl-3,4,4a,5-tetrahydrocyclopenta[d][1,2]oxasilin6(1H)-one (6k)

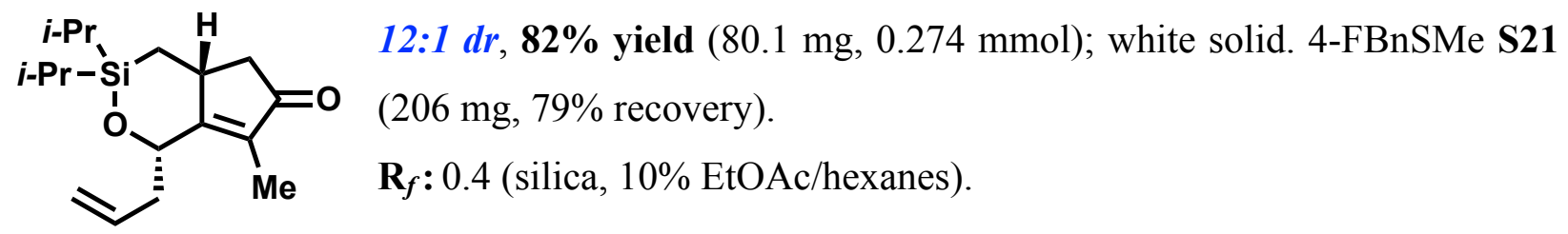

${ }^{1}$ H NMR (400 MHz, $\left.\mathbf{C D C l}_{3}\right): \delta 5.96(\mathrm{ddt}, J=17.1,10.3,6.6 \mathrm{~Hz}, 1 \mathrm{H}), 5.19-5.10$ (m, 2H), 4.94 $(\mathrm{d}, J=10.6 \mathrm{~Hz}, 1 \mathrm{H}), 3.08-2.97(\mathrm{~m}, 1 \mathrm{H}), 2.72(\mathrm{dd}, J=18.4,6.1 \mathrm{~Hz}, 1 \mathrm{H}), 2.61-2.52(\mathrm{~m}, 1 \mathrm{H})$, $2.45-2.35(\mathrm{~m}, 1 \mathrm{H}), 2.04(\mathrm{dd}, J=18.4,2.9 \mathrm{~Hz}, 1 \mathrm{H}), 1.69(\mathrm{~s}, 3 \mathrm{H}), 1.25(\mathrm{dd}, J=14.4,4.3 \mathrm{~Hz}, 1 \mathrm{H})$, $1.11-1.01(\mathrm{~m}, 8 \mathrm{H}), 1.01-0.88(\mathrm{~m}, 6 \mathrm{H}), 0.55(\mathrm{t}, J=14.5 \mathrm{~Hz}, 1 \mathrm{H})$.

${ }^{13}$ C NMR (101 MHz, $\left.\mathbf{C D C l}_{3}\right): \delta$ 207.91, 175.35, 134.98, 133.48, 117.36, 74.33, 45.42, 41.68, $36.70,18.07,17.77,17.30,17.11,15.16,13.94,12.52,9.19$.

IR (neat, $\mathbf{c m}^{-1}$ ): 2942, 2865, 1702, 1638, 1463, 1410, 1382, 1288, 1221, 1141, 1056, 989, 913, $882,802,757,736,711$.

HRMS ( $\left.\mathbf{C I}^{+}, \boldsymbol{m} / \boldsymbol{z}\right)$ : calculated for $\mathrm{C}_{17} \mathrm{H}_{29} \mathrm{O}_{2} \mathrm{Si}[\mathrm{M}+\mathrm{H}]^{+}$293.19313, found 293.19334. 


\section{Enantioenriched Substrates}

\section{(R)-1-phenylhex-5-en-1-yn-3-ol (-)-S19}

Propargyl Alcohol (-)-S19 was synthesized following a published procedure by H.C. Brown. ${ }^{28 \mathrm{a}-\mathrm{b}}$ Spectral data is in agreement with those previously reported in the literature. ${ }^{28 \mathrm{c}}$

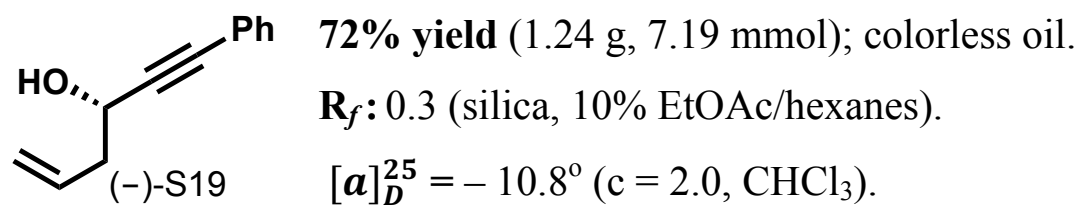

Racemic

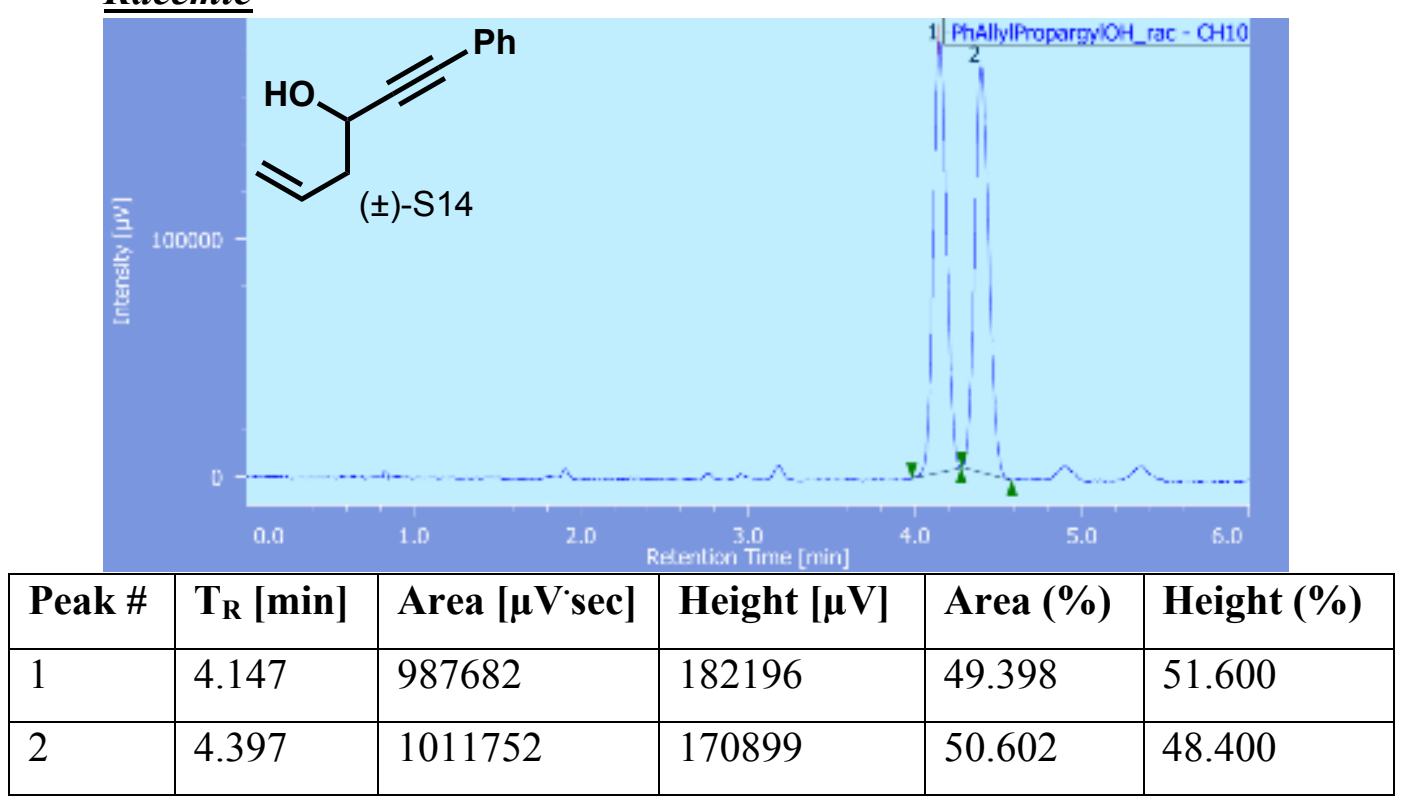

\section{Enantioenriched}

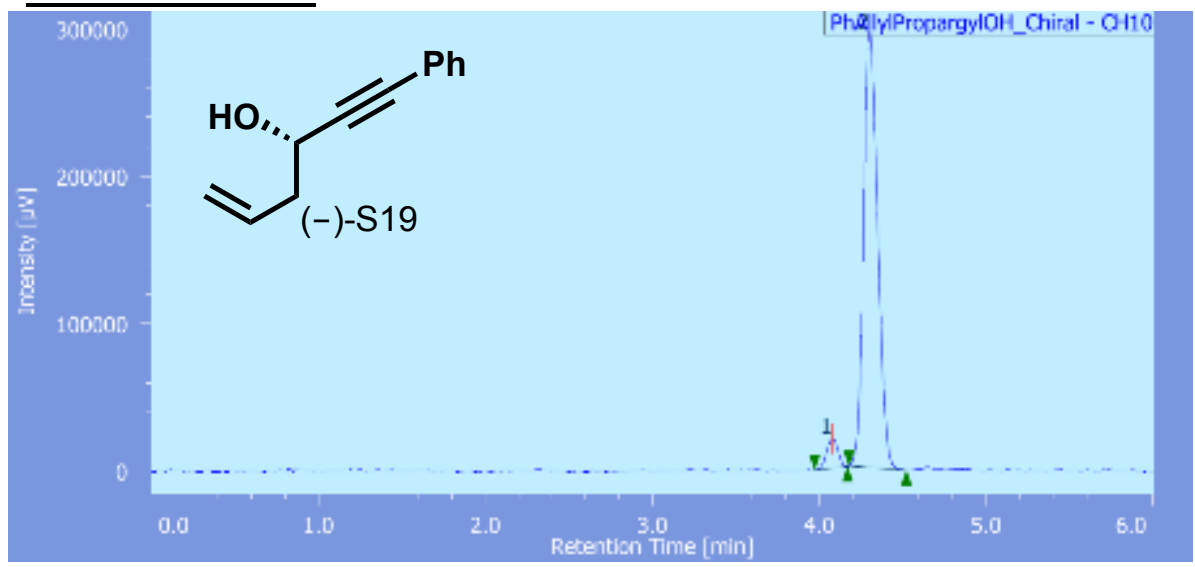

\begin{tabular}{|l|l|l|l|l|l|}
\hline Peak \# & $\mathbf{T}_{\mathbf{R}}[\mathbf{m i n}]$ & Area $[\boldsymbol{\mu V} \mathbf{s e c}]$ & Height $[\boldsymbol{\mu V}]$ & Area (\%) & Height (\%) \\
\hline 1 & 4.08 & 94266 & 20001 & 5.120 & 6.311 \\
\hline 2 & 4.302 & 1747019 & 296932 & 94.880 & 93.689 \\
\hline
\end{tabular}




\section{Allyldiisopropyl((1-phenylhex-5-en-1-yn-3-yl)oxy)silane (-)-5g}

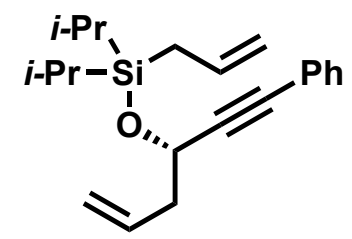

81\% yield (0.768 g, $2.35 \mathrm{mmol})$; colorless oil.

$\mathbf{R}_{f}: 0.8$ (silica, $1 \%$ EtOAc/hexanes).

$$
[a]_{D}^{25}=-50.5^{\circ}\left(\mathrm{c}=2.0, \mathrm{CHCl}_{3}\right)
$$

${ }^{1}$ H NMR (400 MHz, $\mathbf{C D C l}_{3}$ ): $\delta 7.44$ - 7.39 (m, 2H), $7.34-7.28$ (m, 3H), $6.02-5.88$ (m, 2H), $5.22-5.11(\mathrm{~m}, 2 \mathrm{H}), 4.99(\mathrm{ddt}, J=16.9,3.7,1.8 \mathrm{~Hz}, 1 \mathrm{H}), 4.88$ (ddt, $J=10.1,2.2,1.1 \mathrm{~Hz}, 1 \mathrm{H})$, $4.71(\mathrm{t}, J=6.4 \mathrm{~Hz}, 1 \mathrm{H}), 2.59-2.51(\mathrm{~m}, 2 \mathrm{H}), 1.83(\mathrm{dt}, J=8.1,1.4 \mathrm{~Hz}, 2 \mathrm{H}), 1.15-1.04(\mathrm{~m}, 14 \mathrm{H})$. ${ }^{13}$ C NMR (101 MHz, CDCl $)$ ): $\delta$ 134.76, 134.08, 131.68, 128.39, 128.31, 123.16, 117.88, 113.95, $90.62,84.83,77.48,77.16,76.84,63.54,43.50,19.38,17.73,17.67,17.65,12.99,12.93$.

IR (neat, $\mathbf{c m}^{-1}$ ): 3079, 2943, 2866, 1630, 1490, 1463, 1342, 1159, 1084, 993, 914, 882, 754, 689. HRMS $\left(\mathbf{C I}^{+}, \boldsymbol{m} / \boldsymbol{z}\right)$ : calculated for $\mathrm{C}_{21} \mathrm{H}_{31} \mathrm{OSi}[\mathrm{M}+\mathrm{H}]^{+}$327.21387, found 327.21409.

\section{1-Allyl-3,3-diisopropyl-7-phenyl-3,4,4a,5-tetrahydrocyclopenta[d][1,2] ]oxasilin-6(1H)-one $(-)-6 g$}

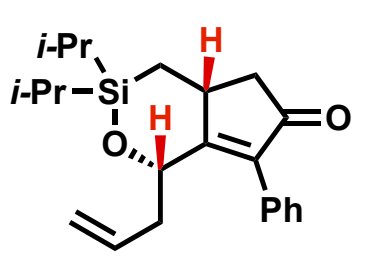

$>20: 1 \mathrm{dr}, 98.5: 1.5 \mathrm{er}, \mathbf{9 2 \%}$ yield, (103.0 mg, $0.291 \mathrm{mmol})$; white solid.

$\mathbf{R}_{\boldsymbol{f}}: 0.4$ (silica, 10\% EtOAc/hexanes). 4-FBnSMe S21 (222 mg, 85\% recovery).

$[\boldsymbol{a}]_{\boldsymbol{D}}^{\mathbf{2 5}}=-22.0^{\mathrm{o}}\left(\mathrm{c}=1.0, \mathrm{CHCl}_{3}\right)$.

Chiral SFC: $\left(\mathrm{OD}-\mathrm{H}, 4 \mathrm{~mL} / \mathrm{min}, 0.1 \%\right.$ IPA in $\left.\mathrm{CO}_{2}, \lambda=254 \mathrm{~nm}\right): \mathrm{t}_{\mathrm{R}}($ major $)=4.14 \mathrm{~min}, \mathrm{t}_{\mathrm{R}}$ (minor) $=4.55 \mathrm{~min}$.

${ }^{1}$ H NMR (400 MHz, $\left.\mathbf{C D C l}_{3}\right): \delta 7.43-7.27$ (m, 3H), $7.22-7.16(\mathrm{~m}, 2 \mathrm{H}), 5.76$ (ddt, $J=17.0$, $10.2,6.7 \mathrm{~Hz}, 1 \mathrm{H}), 5.30$ (d, $J=10.0 \mathrm{~Hz}, 1 \mathrm{H}), 4.94(\mathrm{dd}, J=10.2,1.8 \mathrm{~Hz}, 1 \mathrm{H}), 4.76$ (dt, $J=17.1,1.7$ $\mathrm{Hz}, 1 \mathrm{H}), 3.31-3.22(\mathrm{~m}, 1 \mathrm{H}), 2.93(\mathrm{dd}, J=18.6,6.4 \mathrm{~Hz}, 1 \mathrm{H}), 2.24(\mathrm{dd}, J=18.6,2.7 \mathrm{~Hz}, 1 \mathrm{H}), 2.19$ - $2.01(\mathrm{~m}, 2 \mathrm{H}), 1.37(\mathrm{dd}, J=14.7,4.4 \mathrm{~Hz}, 1 \mathrm{H}), 1.18-1.09(\mathrm{~m}, 7 \mathrm{H}), 1.08-0.90(\mathrm{~m}, 7 \mathrm{H}), 0.68(\mathrm{t}$, $J=14.4 \mathrm{~Hz}, 1 \mathrm{H})$.

${ }^{13}$ C NMR (101 MHz, $\left.\mathbf{C D C l}_{3}\right): \delta 205.95,177.78,138.45,135.04,132.29,128.87,128.63,128.13$, $117.05,77.48,77.16,76.84,74.52$, 46.06, 40.93, 36.84, 18.08, 17.78, 17.31, 17.12, 15.17, 14.29, 12.66 .

IR (neat, $\mathbf{c m}^{-1}$ ): 2939, 2864, 1696, 1463, 1407, 1292, 1141, 1123, 1082, 916, 882, 807, 758, 698. HRMS ( $\left.\mathbf{C I}^{+}, \boldsymbol{m} / \boldsymbol{z}\right)$ : calculated for $\mathrm{C}_{22} \mathrm{H}_{31} \mathrm{O}_{2} \mathrm{Si}[\mathrm{M}+\mathrm{H}]^{+}$355.20878, found 355.20860. 


\section{$\underline{\text { Racemic }}$}

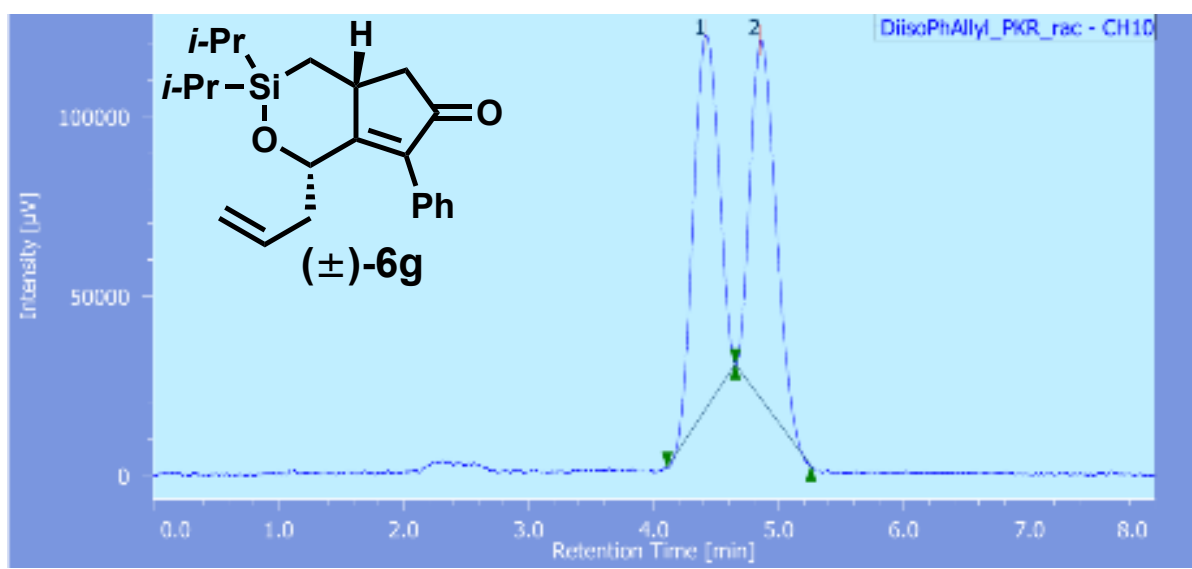

\begin{tabular}{|l|l|l|l|l|l|}
\hline Peak \# & $\mathbf{T}_{\mathbf{R}}[\mathbf{m i n}]$ & Area $[\boldsymbol{\mu V} \cdot \mathbf{s e c}]$ & Height $[\boldsymbol{\mu V}]$ & Area (\%) & Height $(\%)$ \\
\hline 1 & 4.420 & 1455085 & 103796 & 50,611 & 50.921 \\
\hline 2 & 4.857 & 1419931 & 100041 & 49.389 & 49.079 \\
\hline
\end{tabular}

\section{Enantioenriched}

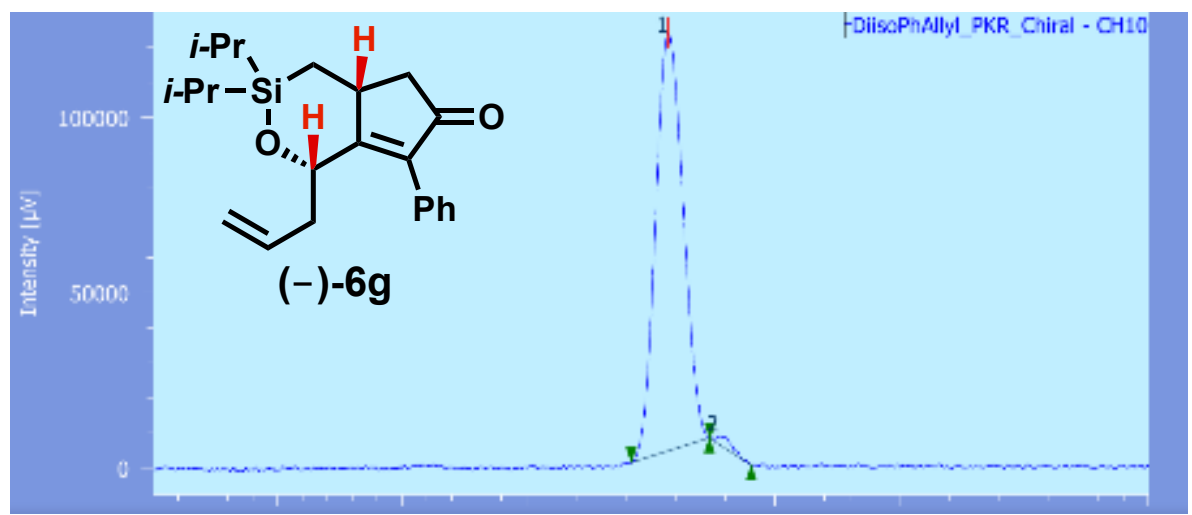

\begin{tabular}{|l|l|l|l|l|l|}
\hline Peak \# & $\mathbf{T}_{\mathbf{R}}[\mathbf{m i n}]$ & Area $[\boldsymbol{\mu V} \mathbf{s e c}]$ & Height $[\boldsymbol{\mu V}]$ & Area (\%) & Height (\%) \\
\hline 1 & 4.140 & 1833000 & 119177 & 98.524 & 97.964 \\
\hline 2 & 4.547 & 27469 & 2477 & 1.476 & 2.036 \\
\hline
\end{tabular}




\section{Functionalization of Siloxy-Tethered Cyclopentenones}

\section{(1S,4aR,6R)-1-allyl-3,3-diisopropyl-7-phenyl-1,3,4,4a,5,6- \\ hexahydrocyclopenta[d][1,2] oxasilin-6-ol (7a)}

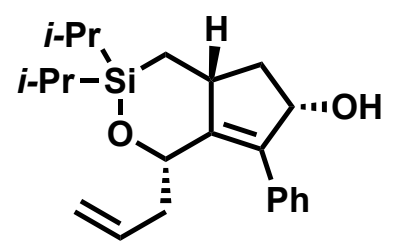

A flame-dried $25 \mathrm{~mL}$ round bottom flask equipped with a magnetic stirbar was placed under inert atmosphere $\left(\mathrm{N}_{2}\right)$ and sequentially charged enone 6g (100 mg, $0.28 \mathrm{mmol}, 1.00$ equiv), MeOH/THF (4:1, 8:2 mL), and cerium(III) chloride heptahydrate $\left(209 \mathrm{mg}, 0.56 \mathrm{mmol}, 2.00\right.$ equiv) at $0{ }^{\circ} \mathrm{C}$. After stirring the reaction mixture for 10 minutes, $\mathrm{NaBH}_{4}(10.3 \mathrm{mg}, 0.34 \mathrm{mmol}, 1.20$ equiv) was added in one portion. The reaction was stirred for 30 minutes at $0{ }^{\circ} \mathrm{C}$. TLC analysis indicated the consumption of $6 \mathbf{g}$ at which time the reaction was quenched with water $(20 \mathrm{~mL})$. The aqueous phase was extracted with EtOAc $(3 \times 20 \mathrm{~mL})$ and the combined organic phases were washed with brine $(25$ $\mathrm{mL}$ ), dried with $\mathrm{MgSO}_{4}$, filtered, and concentrated under reduced pressure. Flash chromatography (silica, 5\% EtOAc/hexanes) provided $7 \mathbf{a}(63.7 \mathrm{mg}, 0.18 \mathrm{mmol}, 63 \%$ yield) as a viscous colorless oil.

$\mathbf{R}_{f}: 0.4$ (silica, $10 \%$ EtOAc/hexanes).

${ }^{1}$ H NMR (400 MHz, CDCl $): \delta 7.55-7.19(\mathrm{~m}, 5 \mathrm{H}), 5.75(\mathrm{dddd}, J=17.3,10.3,7.2,6.3 \mathrm{~Hz}, 1 \mathrm{H})$, 4.89 (ddt, $J=10.3,2.3,1.2 \mathrm{~Hz}, 1 \mathrm{H}), 4.79-4.70$ (m, 3H), $2.94-2.83$ (m, 1H), 2.73 (dt, $J=12.6$, $7.3 \mathrm{~Hz}, 1 \mathrm{H}), 2.13-1.98(\mathrm{~m}, 2 \mathrm{H}), 1.77$ (bs, $1 \mathrm{H}), 1.44$ (dt, $J=13.0,6.6 \mathrm{~Hz}, 1 \mathrm{H}), 1.21$ (dd, $J=14.4$, $4.7 \mathrm{~Hz}, 1 \mathrm{H}), 1.10-0.98$ (m, 14H), 0.67 (dd, $J=14.5,13.5 \mathrm{~Hz}, 1 \mathrm{H})$.

${ }^{13}$ C NMR (101 MHz, CDCl 3 ): $\delta$ 146.12, 137.71, 136.92, 136.11, 128.95, 128.54, 127.40, 116.24, 79.60, 73.56, 44.30, 42.72, 41.00, 18.14, 17.88, 17.43, 17.29, 16.08, 14.69, 12.79 .

IR (neat, $\mathbf{~ c m}^{-1}$ ): 3347, 3074, 2941, 2921, 2891, 2864, 1640, 1599, 1492, 1463, 1087, 990, 912, $882,754,699$.

HRMS (CI $\left.{ }^{+}, \boldsymbol{m} / \boldsymbol{z}\right)$ : calculated for $\mathrm{C}_{22} \mathrm{H}_{31} \mathrm{O}_{2} \mathrm{Si}[\mathrm{M}-\mathrm{H}]^{+}$355.20878, found 355.20911.

(1aS,3aR,7S,7aR)-7-allyl-5,5-diisopropyl-1a-phenyltetrahydro-7Hoxireno[2',3':2,3]cyclopenta[1,2-d][1,2]oxasilin-2(1aH)-one (7b)

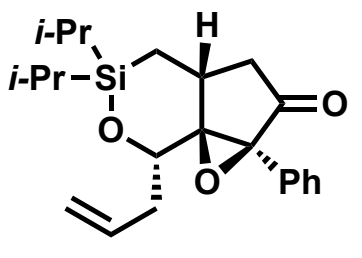

A flame-dried $25 \mathrm{~mL}$ round bottom flask equipped with a magnetic stir bar was sequentially charged with enone $\mathbf{6 g}$ ( $100 \mathrm{mg}, 0.28 \mathrm{mmol}, 1.00$ equiv), and $\mathrm{MeOH} / \mathrm{THF}(3: 1,6: 2 \mathrm{~mL})$. The solution was cooled to $0{ }^{\circ} \mathrm{C}$ and $50 \%$ aqueous $\mathrm{H}_{2} \mathrm{O}_{2}$ solution $(0.26 \mathrm{~mL}, 191 \mathrm{mg}, 5.64 \mathrm{mmol}, 20.0$ equiv) was 
added dropwise, immediately followed by the dropwise addition of a $4 \mathrm{M}$ aqueous $\mathrm{NaOH}$ solution ( $0.69 \mathrm{~mL}, 1.97 \mathrm{mmol}, 7.00$ equiv). The solution was stirred at $0{ }^{\circ} \mathrm{C}$ for $4 \mathrm{~h}$. TLC analysis indicated the consumption of enone $\mathbf{6 g}$ at which time the reaction was quenched with $1 \mathrm{M}$ aqueous $\mathrm{HCl}$ (5 $\mathrm{mL}$ ). The solution was diluted with $\mathrm{CH}_{2} \mathrm{Cl}_{2}$, transferred to a separatory funnel, and washed with water $(2 \times 10 \mathrm{~mL})$. The combined aqueous phases were extracted with $\mathrm{CH}_{2} \mathrm{Cl}_{2}(3 \times 10 \mathrm{~mL})$. The combined organic phases were dried with $\mathrm{Na}_{2} \mathrm{SO}_{4}$, filtered, and concentrated under reduced pressure. Flash chromatography (silica, 1\% EtOAc/hexanes) provided 7b $(67.9 \mathrm{mg}, 0.18 \mathrm{mmol}$, $66 \%$ yield) as a white to clear crystalline solid.

Note: The stereochemistry of the epoxide in $7 \boldsymbol{b}$ is assigned by analogy to that of the expoxide in 7e. Please see 339 for X-ray crystal strutcture of $7 \boldsymbol{e}$.

$\mathbf{R}_{\boldsymbol{f}}: 0.67$ (silica, 10\% EtOAc/hexanes).

${ }^{1}$ H NMR (400 MHz, $\left.\mathbf{C D C l}_{3}\right): \delta 7.44-7.28$ (m, 5H), 5.69 (dddd, $\left.J=17.1,10.2,7.9,5.8 \mathrm{~Hz}, 1 \mathrm{H}\right)$, 4.90 (ddt, $J=10.2,2.3,1.1 \mathrm{~Hz}, 1 \mathrm{H}), 4.84$ (ddt, $J=17.2,2.0,1.6 \mathrm{~Hz}, 1 \mathrm{H}), 4.49$ (dd, $J=10.6,2.2$ Hz, 1H), $2.96(\mathrm{dd}, J=16.5,7.2 \mathrm{~Hz}, 1 \mathrm{H}), 2.89(\mathrm{ddd}, J=12.7,7.3,5.3 \mathrm{~Hz}, 1 \mathrm{H}), 1.98-1.88(\mathrm{~m}$, $2 \mathrm{H}), 1.30-1.15(\mathrm{~m}, 3 \mathrm{H}), 1.15-0.91(\mathrm{~m}, 13 \mathrm{H}), 0.65(\mathrm{dd}, J=14.9,12.8 \mathrm{~Hz}, 1 \mathrm{H})$.

${ }^{13}$ C NMR (101 MHz, $\left.\mathbf{C D C l}_{3}\right): \delta 210.36,135.88,129.30,128.74,128.40,128.28,116.33,77.48$, $77.16,76.84,76.15,74.53,68.95,43.64,36.60,34.86,17.83,17.58,17.24,15.21,12.27,11.74$.

IR (neat, $\mathbf{c m}^{-1}$ ): 3082, 2945, 2926, 2866, 1744, 1649, 1462, 1137, 939, 915, 882, 817, 745, HRMS (CI $\left.{ }^{+}, \boldsymbol{m} / \boldsymbol{z}\right)$ : calculated for $\mathrm{C}_{22} \mathrm{H}_{31} \mathrm{O}_{3} \mathrm{Si}[\mathrm{M}+\mathrm{H}]^{+}$371.20370, found 371.20408.

(R)-3-((S)-1-hydroxybut-3-en-1-yl)-4-((hydroxydiisopropylsilyl)methyl)-2-phenylcyclopent2-en-1-one (7c)

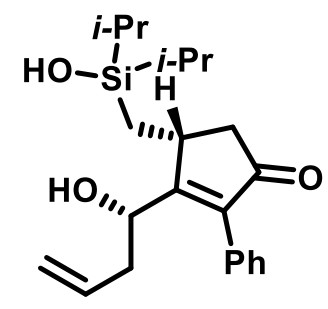

Adapted from a procedure by Gevorgyan and coworkers. ${ }^{31}$ To an ice-cooled $\left(0{ }^{\circ} \mathrm{C}\right)$ stirring solution of $\mathrm{KH}(452 \mathrm{mg}, 3.38 \mathrm{mmol}$, dispersed in mineral oil, $30 \%, 12.0$ equiv) in $3.5 \mathrm{~mL}$ of NMP was added tert-butyl hydroperoxide (3.94 mL, $2.76 \mathrm{mmol}, 10.0$ equiv, $70 \%$ in water) dropwise. The mixture was allowed to warm up to room temperature $\left(25^{\circ} \mathrm{C}\right)$ and stirred for $10 \mathrm{~min}$, then a solution of $\mathbf{6 g}$ (100 mg, $0.28 \mathrm{mmol}, 1.00$ equiv) in $2.8 \mathrm{~mL}$ of NMP was added. The reaction mixture was allowed to stir for $16 \mathrm{~h}$. TBAF (1.41 mL, $1.41 \mathrm{mmol}, 1.0 \mathrm{M}$ solution in THF) was added and the mixture was allowed to stir for another $3 \mathrm{~h}$, then cooled to $0^{\circ} \mathrm{C}$. To the cooled reaction mixture $\mathrm{Na}_{2} \mathrm{~S}_{2} \mathrm{O}_{3} \cdot 5 \mathrm{H}_{2} \mathrm{O}(2.0 \mathrm{~g})$ and of water $(10 \mathrm{~mL})$ were added. The mixture was stirred at $0^{\circ} \mathrm{C}$ for $30 \mathrm{~min}$ followed by addition of $\mathrm{NH}_{4} \mathrm{Cl}$. The aqueous phase was extracted with EtOAc 
$(3 \times 10 \mathrm{~mL})$, the combined organic phases were washed with $\mathrm{H}_{2} \mathrm{O}(4 \times 10 \mathrm{~mL})$, brine $(15 \mathrm{~mL})$, dried with $\mathrm{Na}_{2} \mathrm{SO}_{4}$, filtered, and concentrated under reduced pressure. Flash chromatography (silica, 15\% EtOAc/hexanes) provided $7 \mathrm{c}(53.2 \mathrm{mg}, 0.14 \mathrm{mmol}, 51 \%$ yield) as a white to clear crystalline solid.

$\mathbf{R}_{f}: 0.2$ (silica, 30\% EtOAc/Hexanes)

${ }^{1}$ H NMR (400 MHz, $\left.\mathbf{C D C l}_{3}\right): \delta 7.42-7.37(\mathrm{~m}, 2 \mathrm{H}), 7.35-7.30(\mathrm{~m}, 1 \mathrm{H}), 7.20-7.16(\mathrm{~m}, 2 \mathrm{H})$, 5.70 (dddd, $J=16.8,10.2,7.9,6.4 \mathrm{~Hz}, 1 \mathrm{H}), 5.16-5.07$ (m, 2H), $4.82(\mathrm{dd}, J=9.1,4.0 \mathrm{~Hz}, 1 \mathrm{H})$, 3.36 (ddt, $J=11.5,6.8,2.5 \mathrm{~Hz}, 1 \mathrm{H}$ ), 2.86 (ddd, $J=18.8,6.7,0.8 \mathrm{~Hz}, 1 \mathrm{H}), 2.55-2.39$ (m, 3H), 2.35 (dd, $J=18.8,2.1 \mathrm{~Hz}, 1 \mathrm{H}), 1.57$ (bs, $1 \mathrm{H}), 1.47$ (dd, $J=14.7,2.9 \mathrm{~Hz}, 1 \mathrm{H}), 1.11-1.03$ (m, $14 \mathrm{H}), 0.69$ (dd, $J=14.7,11.3 \mathrm{~Hz}, 1 \mathrm{H})$.

${ }^{13}$ C NMR (101 MHz, $\left.\mathbf{C D C l}_{3}\right): \delta 207.45,179.61,139.80,133.91,131.94,129.09,128.59,128.12$, 119.27, 70.16, 44.54, 41.90, 34.90, 29.84, 17.55, 17.53, 17.51, 17.44, 17.31, 13.39, 13.34.

IR (neat, $\mathbf{c m}^{-1}$ ): 3338, 3188, 2957, 2938, 2864, 167, 1625, 1596, 1462, 1343, 1317, 1072, 851, 759,695 .

HRMS (CI $\left.{ }^{+}, \boldsymbol{m} / \mathbf{z}\right)$ : calculated for $\mathrm{C}_{22} \mathrm{H}_{33} \mathrm{O}_{3} \mathrm{Si}[\mathrm{M}+\mathrm{H}]^{+} 373.21935$, found 373.21939 . 


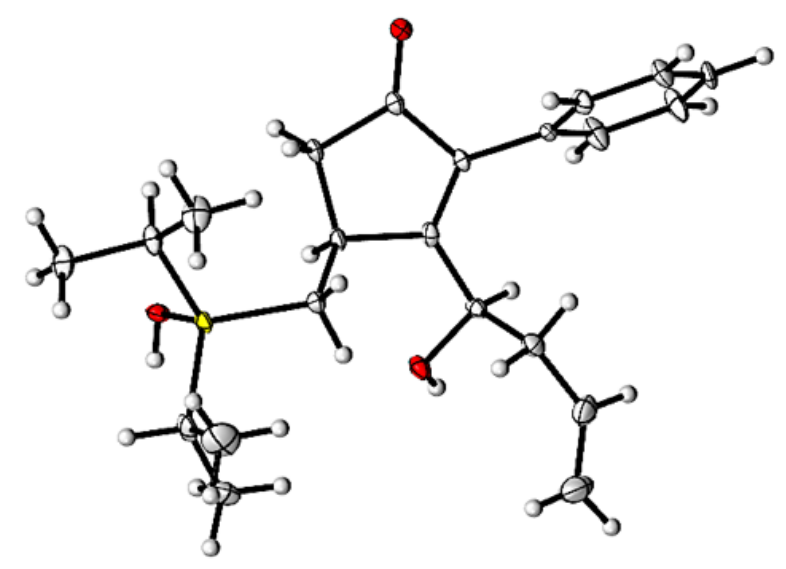

Table S3. Crystal data and structure refinement for (7c).

CCDC Number

Empirical formula

Formula weight

Temperature/K

Crystal system

Space group

a

b

C

$\beta$

Volume

Z

$d_{\text {calc }}$

$\mu$

$\mathrm{F}(000)$

Crystal size, $\mathrm{mm}$

$2 \theta$ range for data collection

Index ranges

Reflections collected

Independent reflections

Data/restraints/parameters

Goodness-of-fit on $\mathrm{F}^{2}$

Final $R$ indexes $[\mathrm{l}>=2 \sigma(\mathrm{I})]$

Final $\mathrm{R}$ indexes [all data]

Largest diff. peak/hole
1944608

$\mathrm{C}_{22} \mathrm{H}_{32} \mathrm{O}_{3} \mathrm{Si}$

372.56

100

monoclinic

$\mathrm{P} 2{ }_{1} / \mathrm{n}$

13.6130(8)Å

$9.2151(5) \AA$

$17.6361(10) \AA$

$99.306(2)^{\circ}$

2183.2(2) $\AA^{3}$

4

$1.133 \mathrm{~g} / \mathrm{cm}^{3}$

$0.125 \mathrm{~mm}^{-1}$

808.0

$0.33 \times 0.14 \times 0.09$

$6.044-50.794^{\circ}$

$-16 \leq \mathrm{h} \leq 16,-11 \leq \mathrm{k} \leq 11,-21 \leq \mathrm{I} \leq 20$

28527

$4007[R$ (int) $=0.0975]$

$4007 / 0 / 241$

1.088

$\mathrm{R}_{1}=0.0571, \mathrm{wR}_{2}=0.0986$

$\mathrm{R}_{1}=0.0839, \mathrm{wR}_{2}=0.1074$

$0.32 /-0.39 \mathrm{e}^{-3}$ 
(R)-3-((S)-1-hydroxybut-3-en-1-yl)-4-(hydroxymethyl)-2-phenylcyclopent-2-en-1-one (7d)

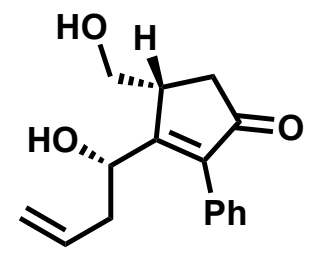

Adapted from a procedure by Maiti and coworkers. ${ }^{32}$ To an pre-cooled $\left(0^{\circ} \mathrm{C}\right)$ stirring solution of $\mathrm{KF}\left(0.25 \mathrm{~g}, 4.23 \mathrm{mmol}, 5.00\right.$ equiv) and $\mathrm{KHCO}_{3}(0.42 \mathrm{~g}$, $4.23 \mathrm{mmol}, 5.00$ equiv) in MeOH:THF (1:1, 2.1:2.1 mL), was added 30\% aqueous $\mathrm{H}_{2} \mathrm{O}_{2}(3.29 \mathrm{~mL}, 1.43 \mathrm{~g}, 42.3 \mathrm{mmol}, 50$ equiv) dropwise under an inert atmosphere $\left(\mathrm{N}_{2}\right)$. In a separate flame-dried $10 \mathrm{~mL}$ vial, $6 \mathrm{~g}(0.30 \mathrm{~g}, 0.84 \mathrm{mmol}, 1.00$ equiv) was diluted in THF $(0.3 \mathrm{~mL})$ and added dropwise at $0{ }^{\circ} \mathrm{C}$. The reaction was allowed to warm to room temperature, fitted with a reflux condenser, and heated to $60{ }^{\circ} \mathrm{C}$ for $48 \mathrm{~h}$. The reaction mixture was cooled $\left(0{ }^{\circ} \mathrm{C}\right)$, then $\mathrm{Na}_{2} \mathrm{~S}_{2} \mathrm{O}_{3} \cdot 5 \mathrm{H}_{2} \mathrm{O}(2.0 \mathrm{~g})$ and water $(10 \mathrm{~mL})$ were added. The mixture was stirred at $0^{\circ} \mathrm{C}$ for $30 \mathrm{~min}$ followed by addition of $\mathrm{NH}_{4} \mathrm{Cl}(10 \mathrm{~mL})$. The aqueous phase was extracted with EtOAc $(3 \times 20 \mathrm{~mL})$, the combined organic phases dried over $\mathrm{Na}_{2} \mathrm{SO}_{4}$, filtered, and concentrated under reduced pressure. Flash chromatography (silica, 5\% EtOAc/hexanes) provided the recovered starting material $6 \mathrm{~g}(161 \mathrm{mg}, 0.45 \mathrm{mmol})$. Increasing the solvent polarity $(25 \% \mathrm{EtOAc/hexanes})$ provided diol 7d (42.8 $\mathrm{mg}, 0.17 \mathrm{mmol}, 42 \%$ yield; based on the recovery of starting material $\mathbf{6 g}$ ) as a viscous colorless oil.

$\mathbf{R}_{\boldsymbol{f}}: 0.2$ (silica, $1 \% \mathrm{MeOH} / \mathrm{CH}_{2} \mathrm{Cl}_{2}$ )

${ }^{1}$ H NMR (400 MHz, $\left.\mathbf{C D C l}_{3}\right): \delta 7.43-7.31(\mathrm{~m}, 5 \mathrm{H}), 5.62$ (dddd, $\left.J=16.7,10.1,7.7,6.3 \mathrm{~Hz}, 1 \mathrm{H}\right)$, $5.02(\mathrm{ddt}, J=10.2,1.9,1.0 \mathrm{~Hz}, 1 \mathrm{H}), 4.88(\mathrm{dq}, J=17.1,1.6 \mathrm{~Hz}, 1 \mathrm{H}), 4.10-4.04(\mathrm{~m}, 2 \mathrm{H}), 3.78$ (dd, $J=11.3,3.9 \mathrm{~Hz}, 1 \mathrm{H}), 2.87$ (dddd, $J=8.9,3.7,2.7,1.0 \mathrm{~Hz}, 1 \mathrm{H}), 2.79$ (dd, $J=17.4,8.9 \mathrm{~Hz}$, 1H), 2.67 (bs, 1H), 2.23 (dddt, $J=14.4,6.2,3.0,1.4 \mathrm{~Hz}, 2 \mathrm{H}), 2.16(\mathrm{dd}, J=17.5,0.9 \mathrm{~Hz}, 1 \mathrm{H}), 2.07$ $-1.98(\mathrm{~m}, 1 \mathrm{H})$.

${ }^{13}$ C NMR (101 MHz, $\left.\mathbf{C D C l}_{3}\right): \delta$ 208.42, 134.19, 129.02, 128.77, 128.38, 127.98, 118.62, 75.81, $70.82,68.25,62.98,39.15,37.72,37.49$.

IR (neat, $\mathbf{c m}^{-1}$ ): 3432, 3066, 2927, 1742, 1641, 1502, 1448, 1407, 1281, 1135, 1066, 999, 915, $887,749,732$.

HRMS (CI $\left.{ }^{+}, \boldsymbol{m} / \boldsymbol{z}\right)$ : calculated for $\mathrm{C}_{16} \mathrm{H}_{17} \mathrm{O}_{3}[\mathrm{M}-\mathrm{H}]^{+} 257.11722$, found 257.11741 .

(1aS,3a R,7S,7aR)-7-benzyl-5,5-diisopropyl-1a-phenyltetrahydro-7H-

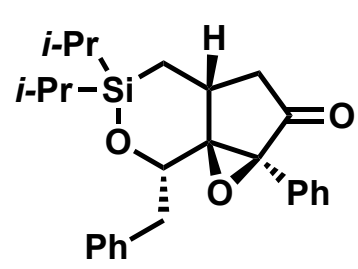
oxireno $\left[2^{\prime}, 3^{\prime}: 2,3\right]$ cyclopenta[1,2-d][1,2]oxasilin-2(1aH)-one (7e)

A flame-dried $25 \mathrm{~mL}$ round bottom flask equipped with a magnetic stir bar was sequentially charged with enone $\mathbf{6 g}$ (100 $\mathrm{mg}, 0.28 \mathrm{mmol}, 1.00$ equiv), 
and $\mathrm{MeOH} / \mathrm{THF}(3: 1,6: 2 \mathrm{~mL})$ under inert atmosphere $\left(\mathrm{N}_{2}\right)$. The solution was cooled to $0{ }^{\circ} \mathrm{C}$ and $50 \%$ aqueous $\mathrm{H}_{2} \mathrm{O}_{2}$ solution ( $0.27 \mathrm{~mL}, 191 \mathrm{mg}, 5.64 \mathrm{mmol}, 20.0$ equiv) was added dropwise, and immediately followed by the dropwise addition of a $4 \mathrm{M}$ aqueous $\mathrm{NaOH}$ solution $(0.69 \mathrm{~mL}, 1.97$ mmol, 7.00 equiv). The solution was stirred at $0{ }^{\circ} \mathrm{C}$ for $4 \mathrm{~h}$. TLC analysis indicated the consumption of enone $\mathbf{6 g}$ at which time the reaction was quenched with $1 \mathrm{M}$ aqueous $\mathrm{HCl}(5 \mathrm{~mL})$. The solution was diluted with $\mathrm{CH}_{2} \mathrm{Cl}_{2}$, transferred to a separatory funnel, and washed with water $(2 \times 10 \mathrm{~mL})$. The combined aqueous phases were extracted with $\mathrm{CH}_{2} \mathrm{Cl}_{2}(3 \times 10 \mathrm{~mL})$. The combined organic phases were dried with $\mathrm{Na}_{2} \mathrm{SO}_{4}$, filtered, and concentrated under reduced pressure. Flash chromatography (silica, 1\% EtOAc/hexanes) provided 7e $(67.9 \mathrm{mg}, 0.18 \mathrm{mmol}, 66 \%$ yield) as a white to clear crystalline solid.

$\mathbf{R}_{\boldsymbol{f}}: 0.63$ (10\% EtOAc/hexanes).

MP: $80-83^{\circ} \mathrm{C}$

${ }^{1}$ H NMR (400 MHz, CDCl 3 ): $\delta 7.48$ - 7.33 (m, 5H), $7.20-7.09$ (m, 3H), $7.05-7.00$ (m, 2H), $4.58(\mathrm{dd}, J=10.6,1.7 \mathrm{~Hz}, 1 \mathrm{H}), 2.99$ (dd, $J=16.7,7.3 \mathrm{~Hz}, 1 \mathrm{H}), 2.90$ (ddd, $J=12.7,7.3,5.3 \mathrm{~Hz}$, 1H), 2.51 (dd, $J=13.7,1.7 \mathrm{~Hz}, 1 \mathrm{H}), 1.93$ (d, $J=16.7 \mathrm{~Hz}, 1 \mathrm{H}), 1.63$ (dd, $J=13.7,10.6 \mathrm{~Hz}, 1 \mathrm{H})$, $1.18(\mathrm{dd}, J=14.9,5.4 \mathrm{~Hz}, 1 \mathrm{H}), 1.11-0.95(\mathrm{~m}, 9 \mathrm{H}), 0.82(\mathrm{dd}, J=35.7,7.5 \mathrm{~Hz}, 7 \mathrm{H}), 0.68(\mathrm{dd}, J=$ $14.9,12.8 \mathrm{~Hz}, 1 \mathrm{H})$.

${ }^{13}$ C NMR (101 MHz, $\left.\mathbf{C D C l}_{3}\right): \delta 210.31,139.79,129.54,129.43,128.83,128.56,128.34,127.90$, 126.04, 76.27, 75.66, 69.08, 43.73, 38.55, 34.97, 17.36, 17.32, 17.18, 15.55, 11.98, 11.71.

IR (neat, $\mathbf{c m}^{-1}$ ): 3030, 2935, 2909, 2864, 1746, 1624, 1493, 1668, 1608, 1383, 1343, 1231, 1131, 1085, 1066, 1019, 938, 883, 809, 779, 762.

HRMS $\left(\mathbf{C I}^{+}, \boldsymbol{m} / \boldsymbol{z}\right)$ : calculated for $\mathrm{C}_{26} \mathrm{H}_{33} \mathrm{O}_{3} \mathrm{Si}[\mathrm{M}+\mathrm{H}]^{+} 421.21935$, found 421.211964 . 


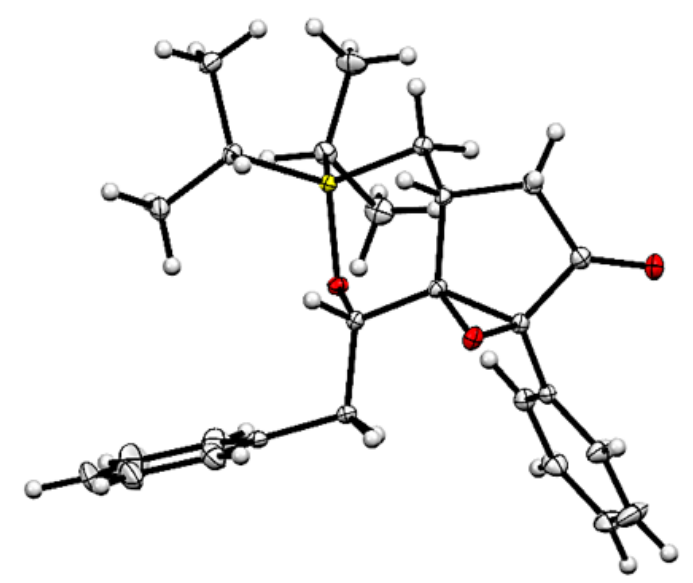

Table S4. Crystal data and structure refinement for (7e).

CCDC Number

Empirical formula

Formula weight

Temperature/K

Crystal system

Space group

a

b

c

a

$\beta$

$Y$

Volume

Z

$\mathrm{d}_{\text {calc }}$

$\mu$

$\mathrm{F}(000)$

Crystal size, $\mathrm{mm}$

$2 \theta$ range for data collection

Index ranges

Reflections collected

Independent reflections

Data/restraints/parameters

Goodness-of-fit on $\mathrm{F}^{2}$

Final $R$ indexes $[l>=2 \sigma(I)]$

Final $R$ indexes [all data]

Largest diff. peak/hole
1944611

$\mathrm{C}_{26} \mathrm{H}_{32} \mathrm{O}_{3} \mathrm{Si}$

420.60

100

triclinic

$\mathrm{P} \overline{1}$

10.5963(4)A

$10.8935(4) \AA$

$12.2184(5) \AA$

$105.9910(10)^{\circ}$

$103.3180(10)^{\circ}$

$114.5990(10)^{\circ}$

$1132.64(8) \AA^{3}$

2

$1.233 \mathrm{~g} / \mathrm{cm}^{3}$

$0.128 \mathrm{~mm}^{-1}$

452.0

$0.35 \times 0.32 \times 0.18$

$5.826-55.078^{\circ}$

$-13 \leq h \leq 13,-14 \leq k \leq 14,-15 \leq l \leq 15$

30106

$5207[\mathrm{R}$ (int) $=0.0276]$

$5207 / 0 / 275$

1.101

$\mathrm{R}_{1}=0.0353, \mathrm{wR}_{2}=0.0902$

$\mathrm{R}_{1}=0.0403, \mathrm{wR}_{2}=0.0938$

$0.35 /-0.39 \mathrm{e}^{-3}$ 


\section{(R)-3-((S)-1-hydroxy-2-phenylethyl)-4-(hydroxymethyl)-2-phenylcyclopent-2-en-1-one (7f)}

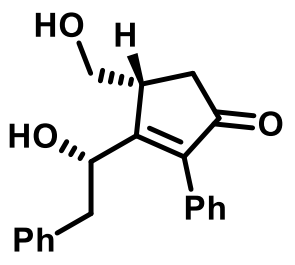

Adapted from a procedure by Jeon and coworkers. ${ }^{33}$ A flame-dried $25 \mathrm{~mL} \mathrm{RBF}$ equipped with a magnetic stir bar was sequentially charged with cesium hydroxide monohydrate (120 mg, $0.99 \mathrm{mmol}, 4.00$ equiv), and DMF (2.50 mL, $0.10 \mathrm{M})$ under inert atmosphere $\left(\mathrm{N}_{2}\right)$. The solution was cooled to $0{ }^{\circ} \mathrm{C}$ and tertbutyl hydroperoxide $(0.74 \mathrm{~mL}, 5.00 \mathrm{M}$ in decane, $3.71 \mathrm{mmol}, 15.0$ equiv), followed by tetrabutylammonium fluoride $(1.24 \mathrm{~mL}, 1.24 \mathrm{mmol}, 1.0 \mathrm{M}$ in THF solution) was added dropwise. In a separate flame-dried $10 \mathrm{~mL}$ pear-shaped flask, cyclopentaoxasilinone $6 \mathrm{~g}(0.30 \mathrm{~g}, 0.84 \mathrm{mmol}$, 1.00 equiv) was diluted with DMF $(0.30 \mathrm{~mL})$ and added dropwise to the reaction mixture at $0{ }^{\circ} \mathrm{C}$. Once addition of $\mathbf{6 g}$ was complete, the reaction was warmed to room temperature $\left(25^{\circ} \mathrm{C}\right)$ and allowed to stir for 17 hours. TLC analysis indicated the consumption of $\mathbf{6 g}$ at which time the reaction was quenched with saturated aqueous sodium thiosulfate $(10 \mathrm{~mL})$ and stirred for $30 \mathrm{~min}$. The aqueous layer was extracted with EtOAc $(3 \times 20 \mathrm{~mL})$, the combined organic phases were washed with aqueous $\mathrm{HCl}(1.0 \mathrm{M}, 20 \mathrm{~mL})$, saturated aqueous sodium bicarbonate $(20 \mathrm{~mL})$, brine $(20 \mathrm{~mL})$, dried over $\mathrm{Na}_{2} \mathrm{SO}_{4}$, filtered, and concentrated under reduced pressure. Flash chromatography (silica, 25\% EtOAc/hexanes) provided $7 \mathbf{f}$ (102 $\mathrm{mg}, 0.33 \mathrm{mmol}, 40 \%$ yield) as a clear viscous oil.

$\mathbf{R}_{\boldsymbol{f}}: 0.54$ (silica, $2 \% \mathrm{MeOH} / \mathrm{CH}_{2} \mathrm{Cl}_{2}$ )

${ }^{1}$ H NMR (400 MHz, $\mathbf{C D C l}_{3}$ ): $\delta 7.46-7.40(\mathrm{~m}, 4 \mathrm{H}), 7.21-7.13(\mathrm{~m}, 3 \mathrm{H}), 6.74(\mathrm{dd}, J=7.7,2.0$ Hz, 2H), 4.10 (ddd, $J=14.1,11.2,2.8 \mathrm{~Hz}, 2 \mathrm{H}), 3.83$ (dd, $J=11.2,4.3 \mathrm{~Hz}, 1 \mathrm{H}), 3.00$ - 2.95 (m, $1 \mathrm{H}), 2.82(\mathrm{dd}, J=17.8,8.9 \mathrm{~Hz}, 1 \mathrm{H}), 2.70(\mathrm{dd}, J=13.9,2.4 \mathrm{~Hz}, 1 \mathrm{H}), 2.52(\mathrm{dd}, J=13.8,10.8 \mathrm{~Hz}$, $1 \mathrm{H}), 2.18(\mathrm{dd}, J=17.8,1.2 \mathrm{~Hz}, 1 \mathrm{H}), 1.26(\mathrm{~s}, 2 \mathrm{H}), 0.93-0.83(\mathrm{~m}, 1 \mathrm{H})$.

${ }^{13}$ C NMR (101 MHz, $\left.\mathbf{C D C l}_{3}\right): \delta$ 208.31, 138.09, 129.17, 129.06, 128.81, 128.64, 128.46, 128.09, $126.74,73.88,68.42,62.97,39.52,38.91,37.55$.

IR (neat, $\mathbf{c m}^{-\mathbf{1}}$ ): 3338, 3030, 2928, 1747, 1604, 1496, 1449, 1403, 1267, 1132, 1060, 1029, 841, $749,733,699$.

HRMS $\left(\mathbf{C I}^{+}, \boldsymbol{m} / \boldsymbol{z}\right)$ : calculated for $\mathrm{C}_{20} \mathrm{H}_{19} \mathrm{O}_{3}[\mathrm{M}-\mathrm{H}]^{+}$307.13287, found 307.13301 . 


\section{References}

1. W. C. Still, M. Kahn, A. Mitra, J. Org. Chem. 1978, 43, 2923 - 2925.

2. APEX3 2016.1-0: Bruker-AXS, Madison, Wisconsin, USA (2016).

3. APEX2 2014.11-0: Bruker-AXS, Madison, Wisconsin, USA (2014).

4. SAINT v8.34A: Bruker-AXS, Madison, Wisconsin, USA (2014).

5. SADABS v2014/5: L. Krause, R. Herbst-Irmer, G.M. Sheldrick, and D. Stalke, J. Appl. Cryst., 2015, 48, 3 - 10.

6. SHELXT v2014/4: G.M Sheldrick, Acta Crystallogr., Sect. A, 2015, 71, 3 - 8.

7. SHELXS-97: G.M. Sheldrick, Acta Crystallogr., Sect. A, 2008, 64, 112 - 122.

8. SHELXL-2018/3: G.M. Sheldrick, Acta Crystallogr., Sect. A, 2015, 71, 3 - 8.

9. C. F. Macrae, P. R. Edgington, P. McCabe, E. Pidcock, G. P. Shields, R. Taylor, M. Towler, J. van de Streek, J. Appl. Cryst. 2006, 39, $453-457$.

10. (S1) M. Hu, J. Li, S. Q. Yao, Org. Lett. 2008, 10, 5529-5531.

11. (S2) S. Aoyagi, T. C. Wang, C. Kibayashi, J. Am. Chem. Soc., 1993, 115, $11393-11409$.

12. (S3) P. Liu, P.S. Wang, Chemistry Letters, 2017, 46, 1190-1192.

13. (S4) Y. Guan, M. P. Lopez-Alberca, Z. Lu, Y. Zhang, A. A. Desai, A. P. Patwardhan, Y. Dai, M. J. Vetticatt, W. D. Wulff, Chem. Eur. J., 2014, 20, 13894 - 13900.

14. (S5) M. Maji, D. Mallick, S. Mondal, A. Anoop, S. Sekhar Bag, A. Basak, E. D. Jemmis, Org. Lett., 2011, 13, $888-891$.

15. (S6) Y. Guan, M. P. Lopez-Alberca, Z. Lu, Y. Zhang, A. A. Desai, A. P. Patwardhan, Y. Dai, M. J. Vetticatt, W. D. Wulff, Chem. Eur. J., 2014, 20, $13894-13900$.

16. (S7) J. Panteleev, R. Y. Huang, E. K. J. Lui, M. Lautens, Org. Lett., 2011, 13, 5314 5317.

17. (S8) K. K. Chan, N. Cohen, J. P. De Noble, A. C. Specian, G. Saucy, J. Org. Chem., 1976, 41, 3497.

18. (S9) M. Barbazanges, C. Meyer, J. Cossy, Org. Lett., 2008, 10, 4489 - 4492.

19. (S10) B. S. Chinta, B., Baire, Eur. J. Org. Chem., 2017, 23, 3381 - 3385.

20. (S11) D. A. Engel, G. B. Dudley, Org. Lett., 2006, 8, 4027 - 4029.

21. (S12) Gao, G., Moore, D., Ru-Gang, X., Pu, L., Org Lett., 2002, 4, 4143 - 4146.

22. (S13) A. Boussonniere, R. Beneteau, N. Zimmermann, J. Lebreton, F. Denes, Chem. Eur. $J ., 2011,17,5613-5627$.

23. (S14) V. Mamane, T. Gress, H. Krause, A. Furstner, J. Am. Chem. Soc., 2004, 126, 8654 -8655 .

24. (S15) V. Gudla, R. Balamurugan, J. Org. Chem., 2011, 76, 9919 - 9933.

25. (S16) K. L. Salazar, K. M. Nicholas, Tetrahedron, 2000, 56, 2211 - 2224.

26. (S17) M. C. Pacheco, V. Gouverneur, Org. Lett., 2005, 7, $1267-1270$.

27. (S18) P. H. Dussault, J. A. Schultz, J. Org. Chem, 1999, 64, 8419 - 8422.

28. (S19) (a) Racherla, U. S.; Brown, H. C. J. Org. Chem. 1991, 56, 401. (b) Jadhav, P. K.; Bhat, K. S.; Perumal, P. T.; Brown, H. C. J. Org. Chem. 1986, 51, 432. (c) S. E. Denmark, T. Wynn, J. Am. Chem Soc., 2001, 123, 6199 - 6200.

29. (S20) 3M Innovative Properties Company; Outrider Technologies, WO 2009/155106, 2009, A1.

30. (S21) H. L. Holland, F. M. Brown, B. G. Larsen, Tetrahedron: Asymmetry, 1995, 6, 1561 $-1567$.

31. M. Parasram, V. O. Laroshenkok, V. Gevorgyan, J. Am. Chem. Soc., 2014, 136, 17926 17929. 
32. U. Dutta, S. Maiti, S. Pimparkar, S. Maiti, L. R. Gahan, E. H. Krenske, D. W. Lupton, D. Maiti, Chem. Sci. 2019, 10, 7426 - 7432.

33. Y. Hua, H. H. Nguyen, W. R. Scaggs, J. Jeon, Org. Lett. 2013, 15, 3412 - 3415. 


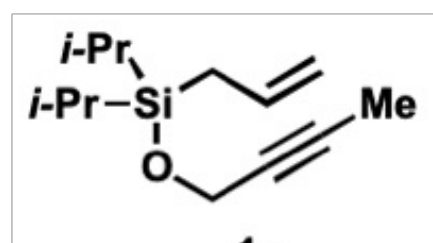

$\frac{m}{u}$
0
$\stackrel{0}{N}$
$\stackrel{i}{I}$

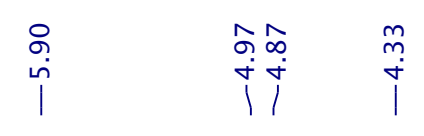

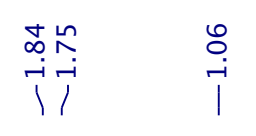

$1 a$

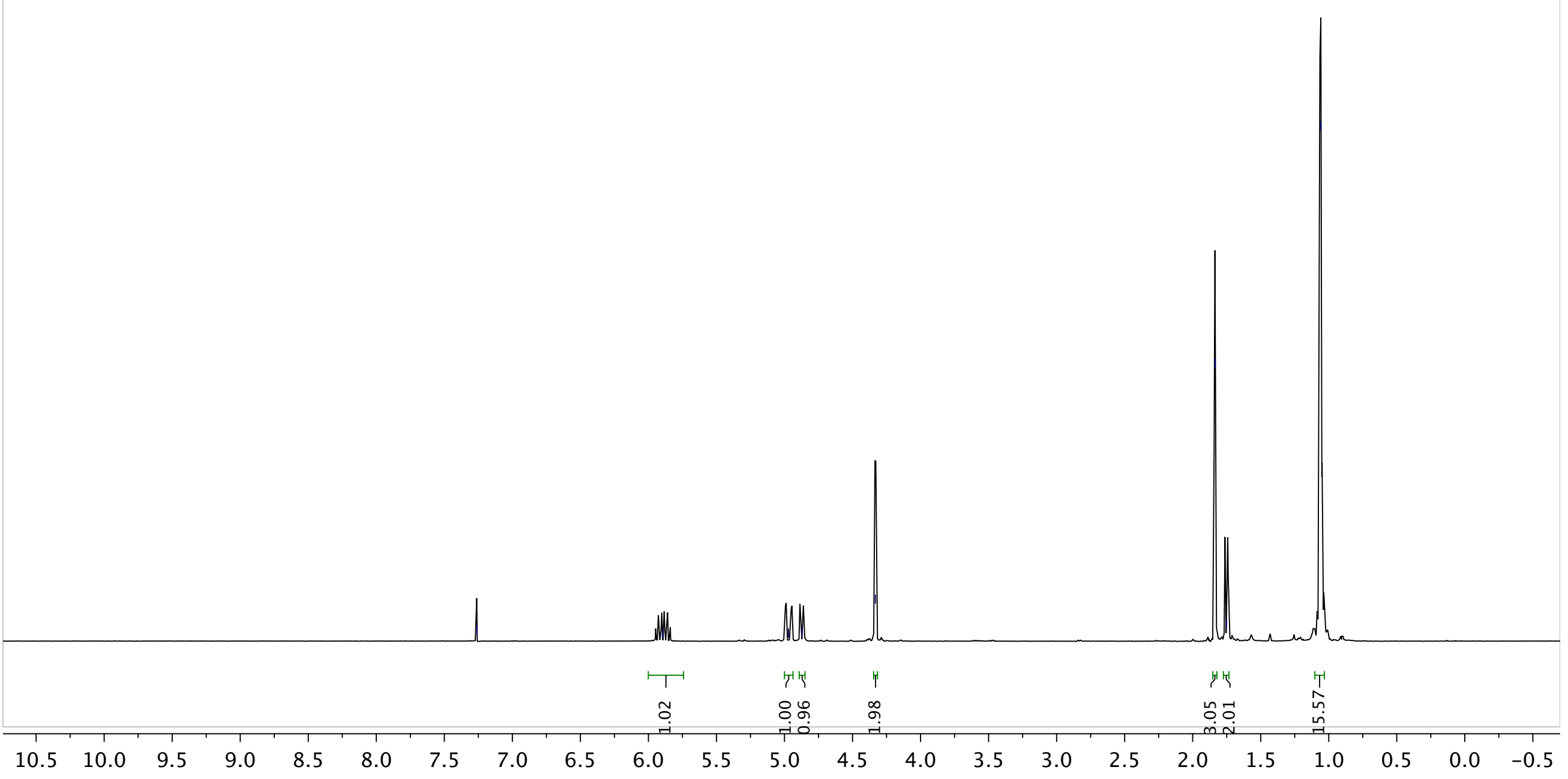




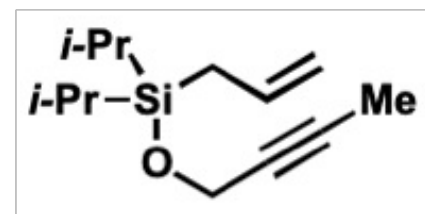

1a

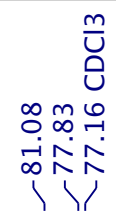

$\begin{array}{lllllllllllllllllllllllll}230 & 220 & 210 & 200 & 190 & 180 & 170 & 160 & 150 & 140 & 130 & 120 & 110 & 100 & 90 & 80 & 70 & 60 & 50 & 40 & 30 & 20 & 10 & 0 & -10\end{array}$




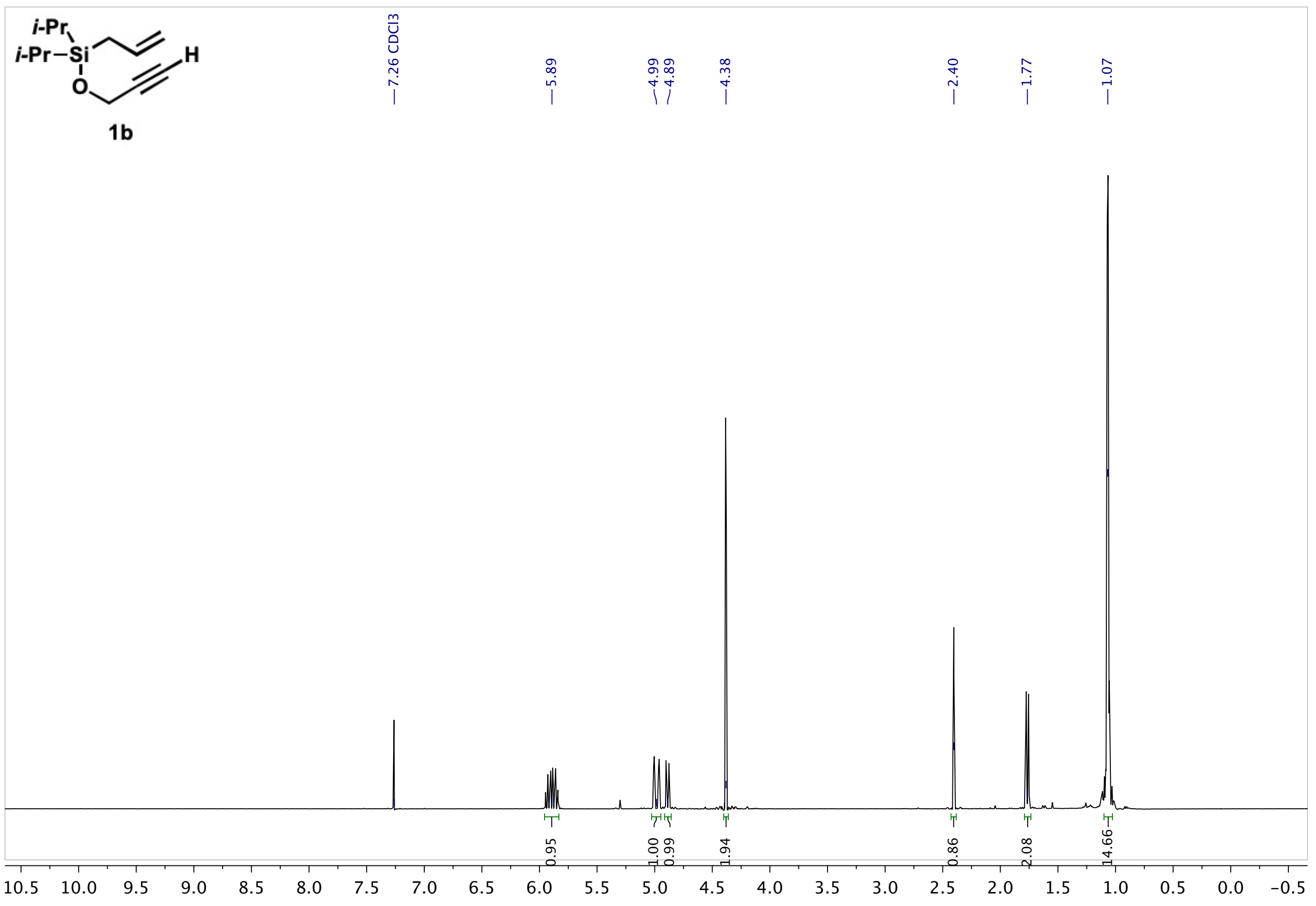


S46

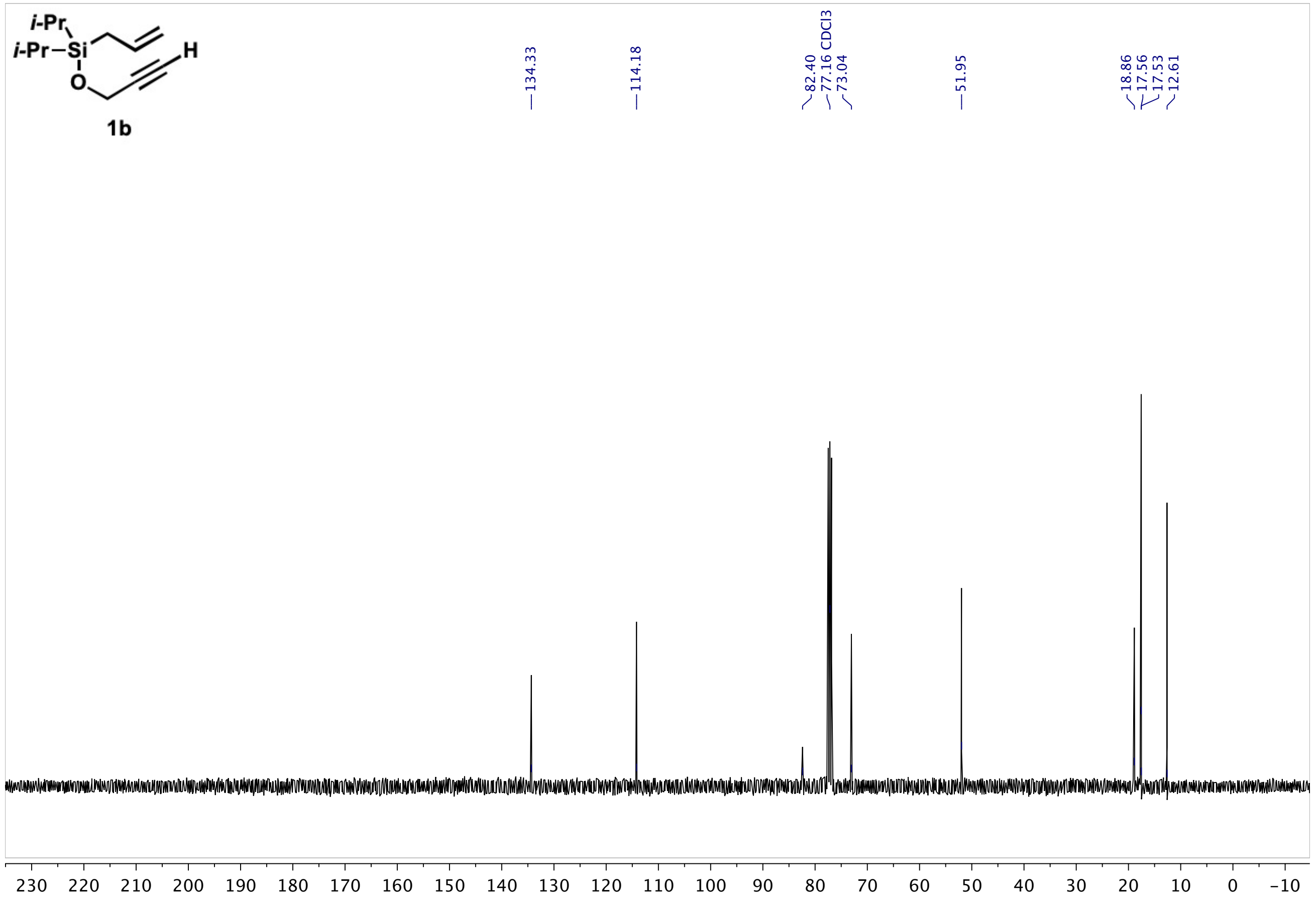


S47

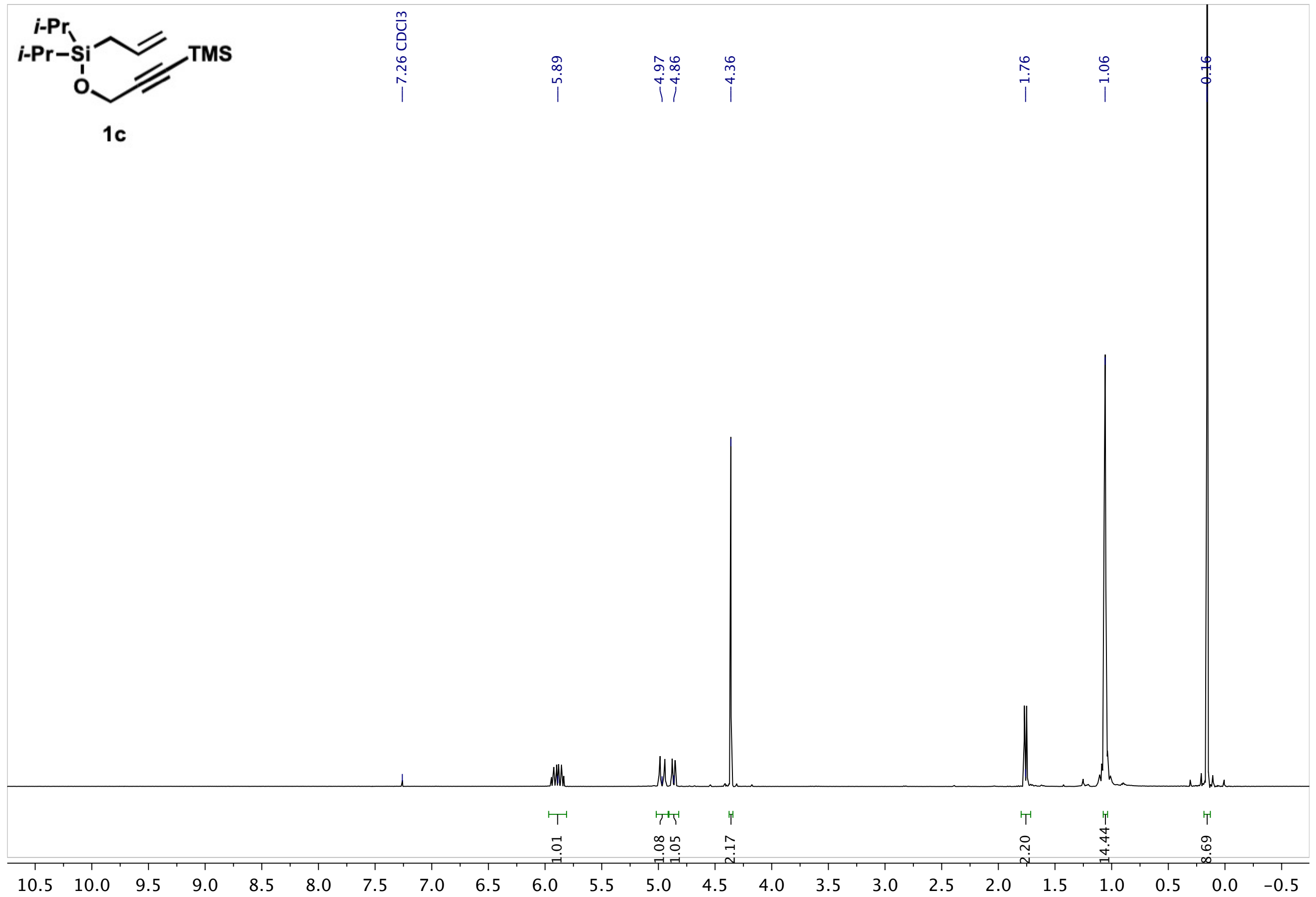


S48

o

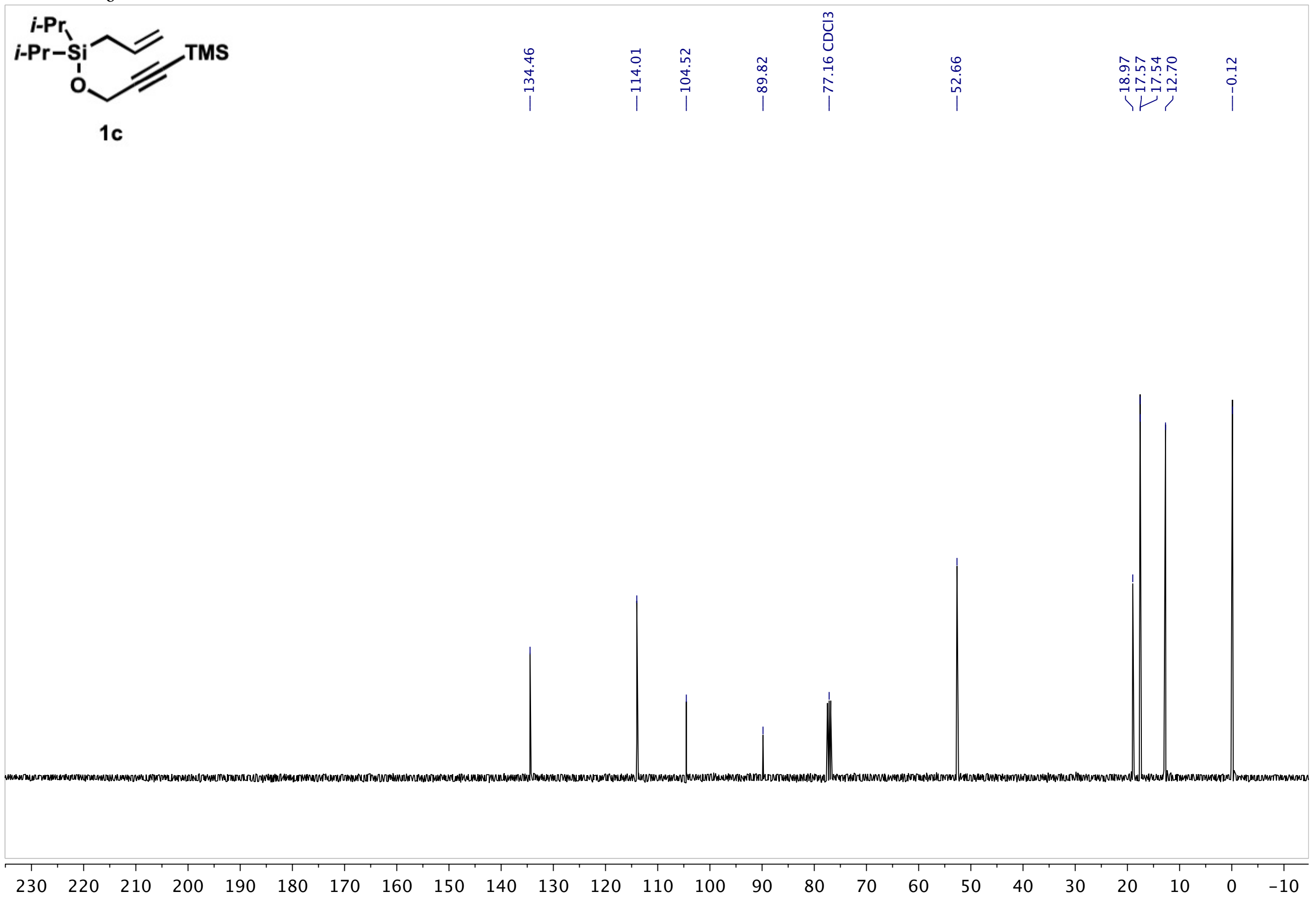




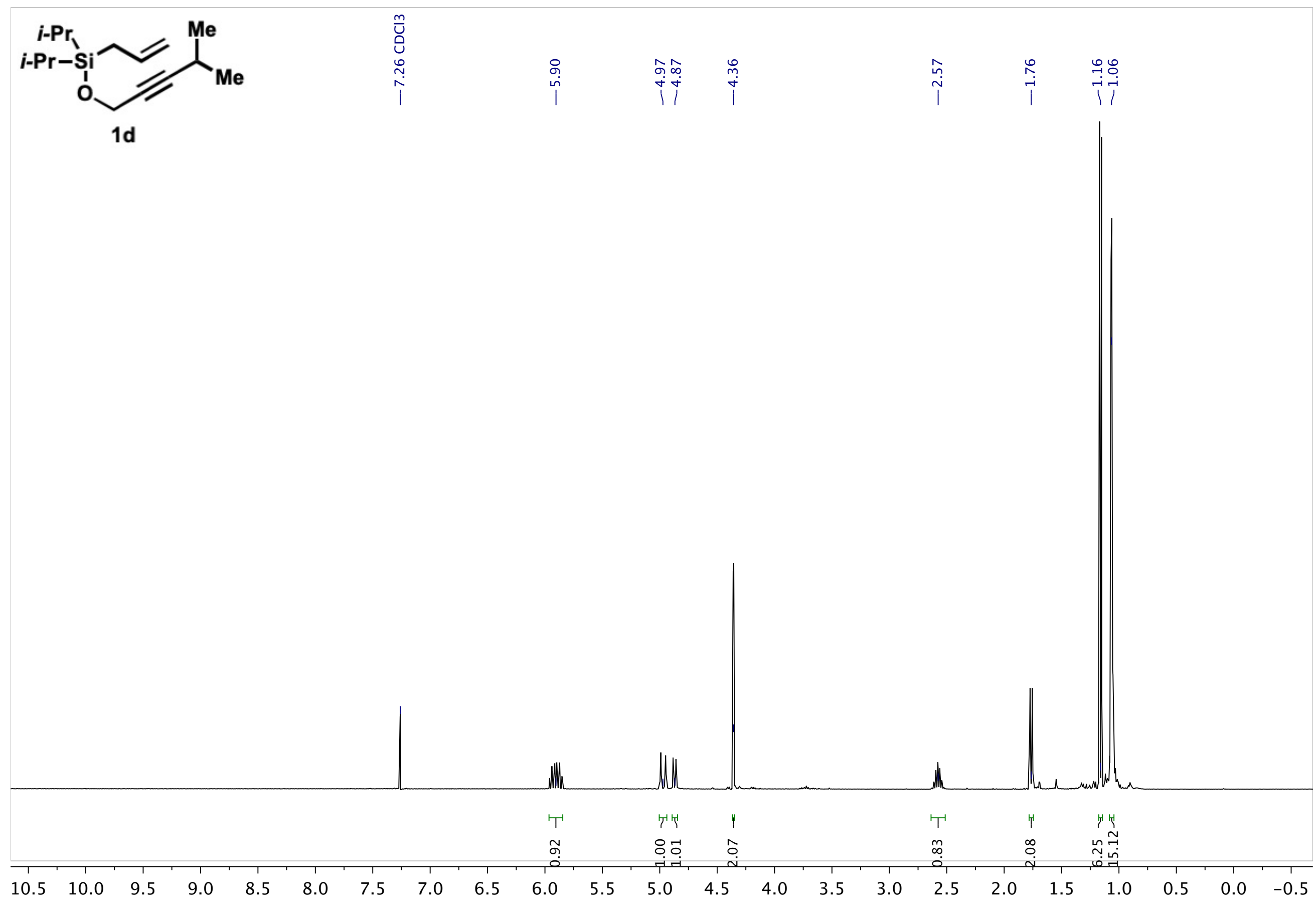




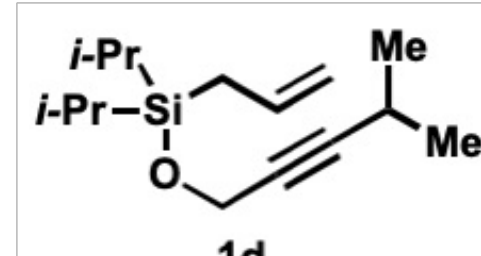

1d

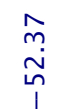

옹ํํ뭉ํ요

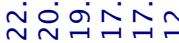

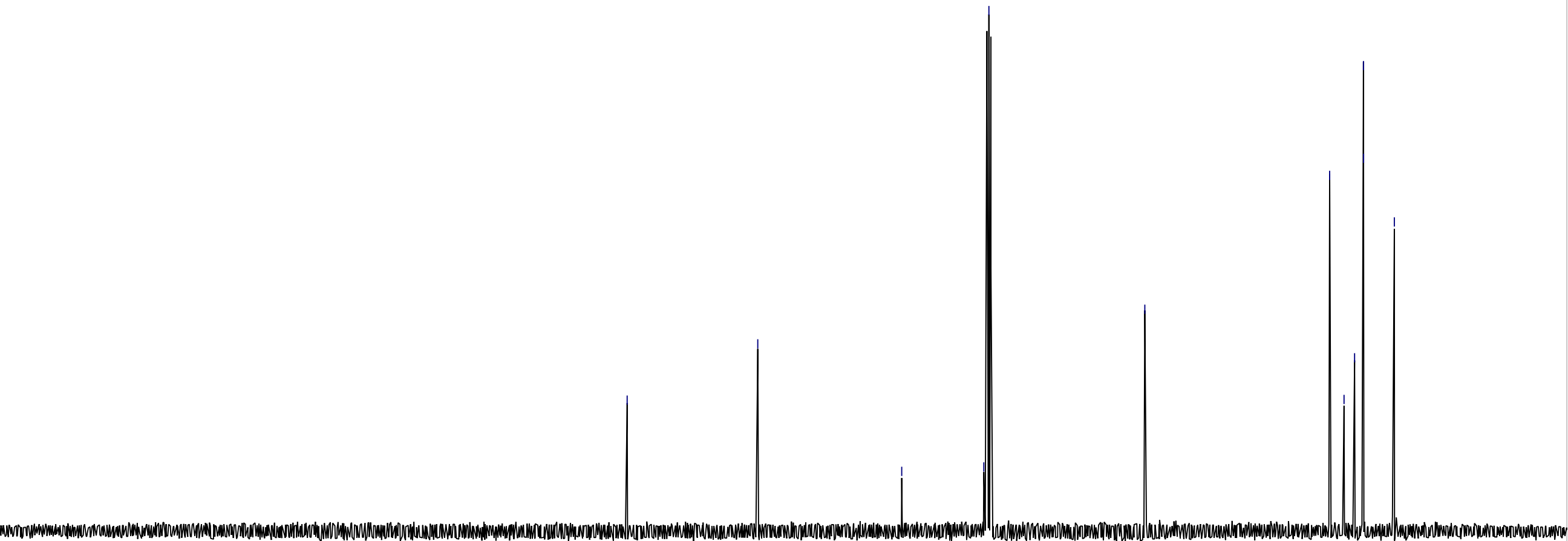


S51

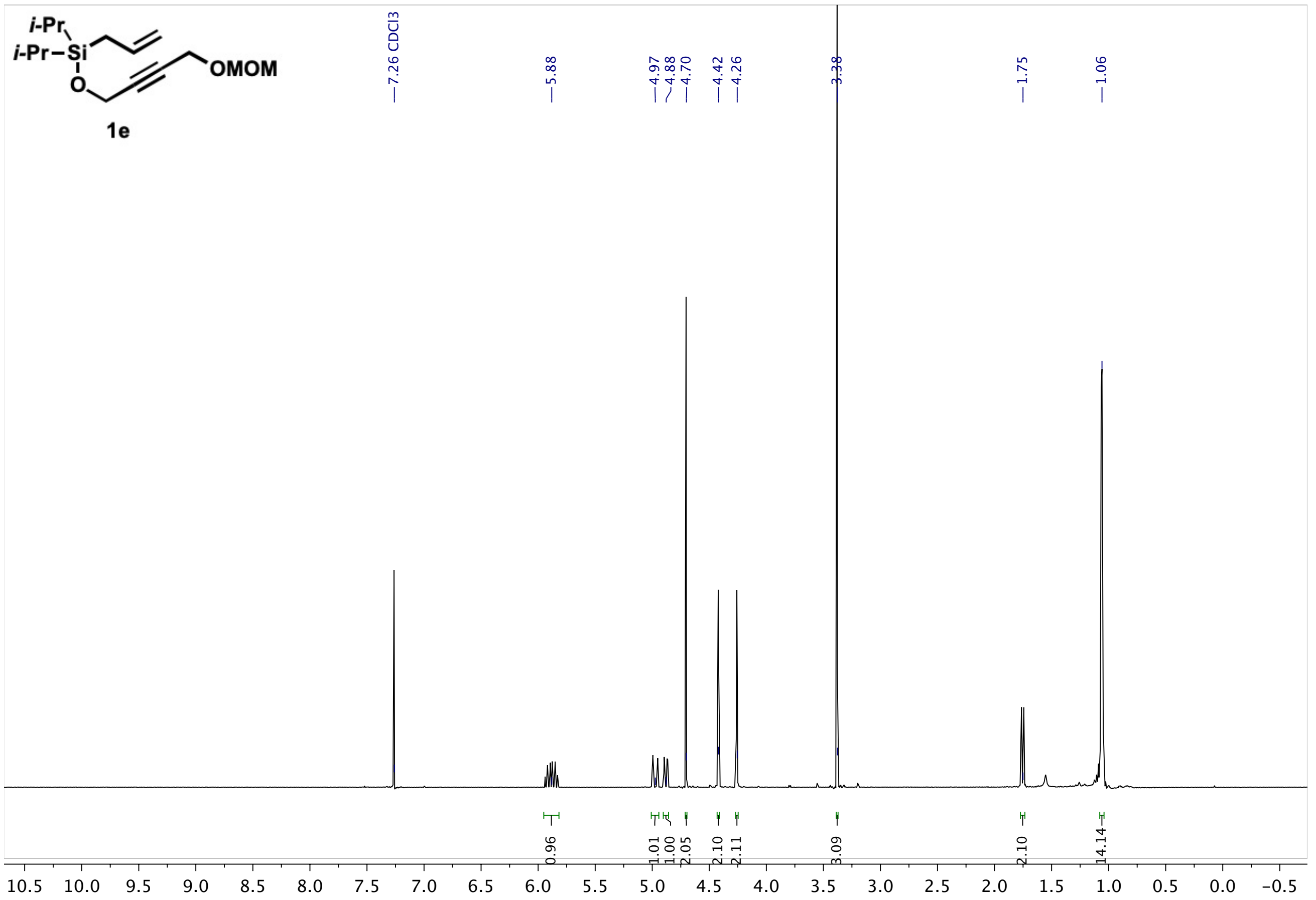




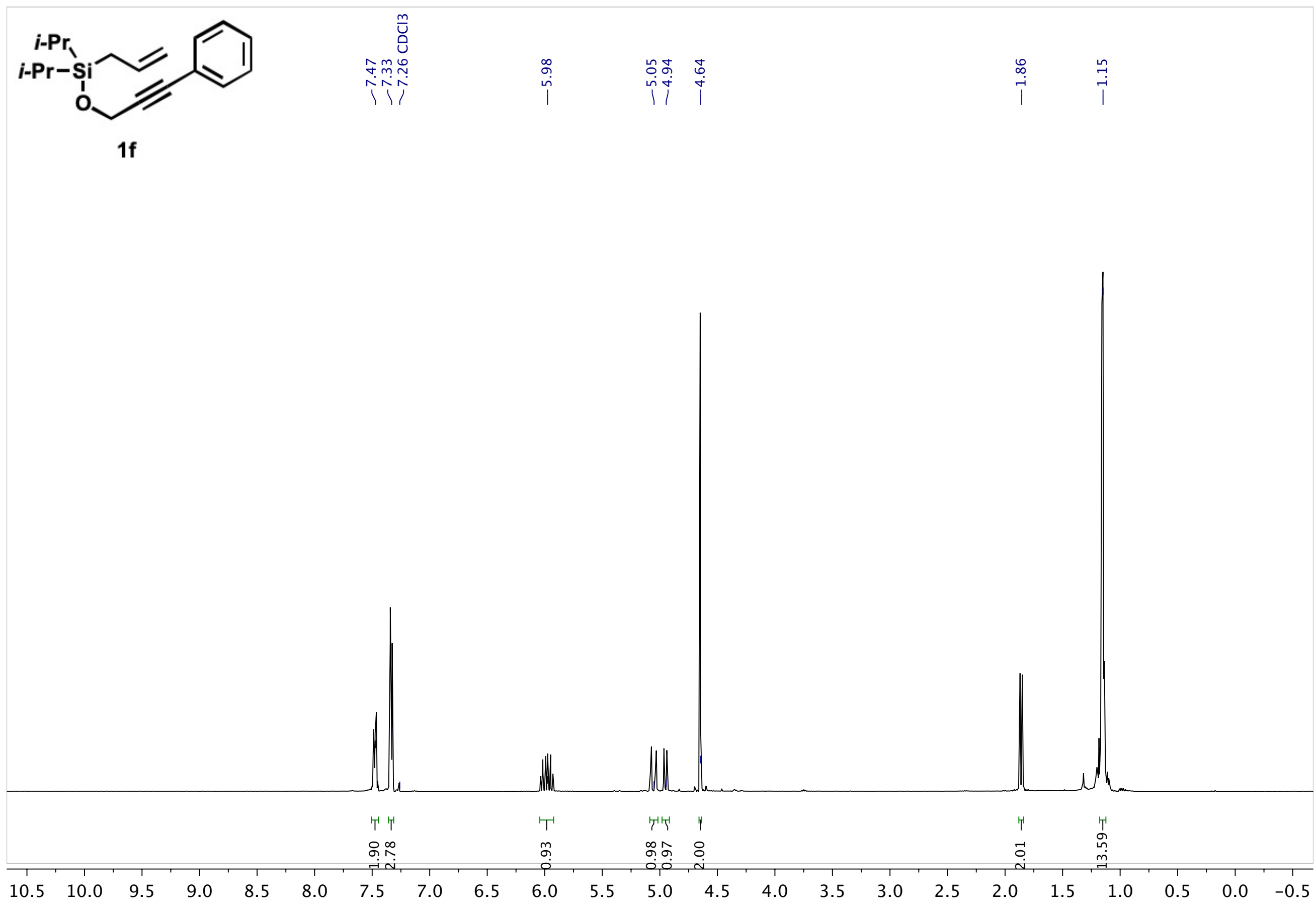

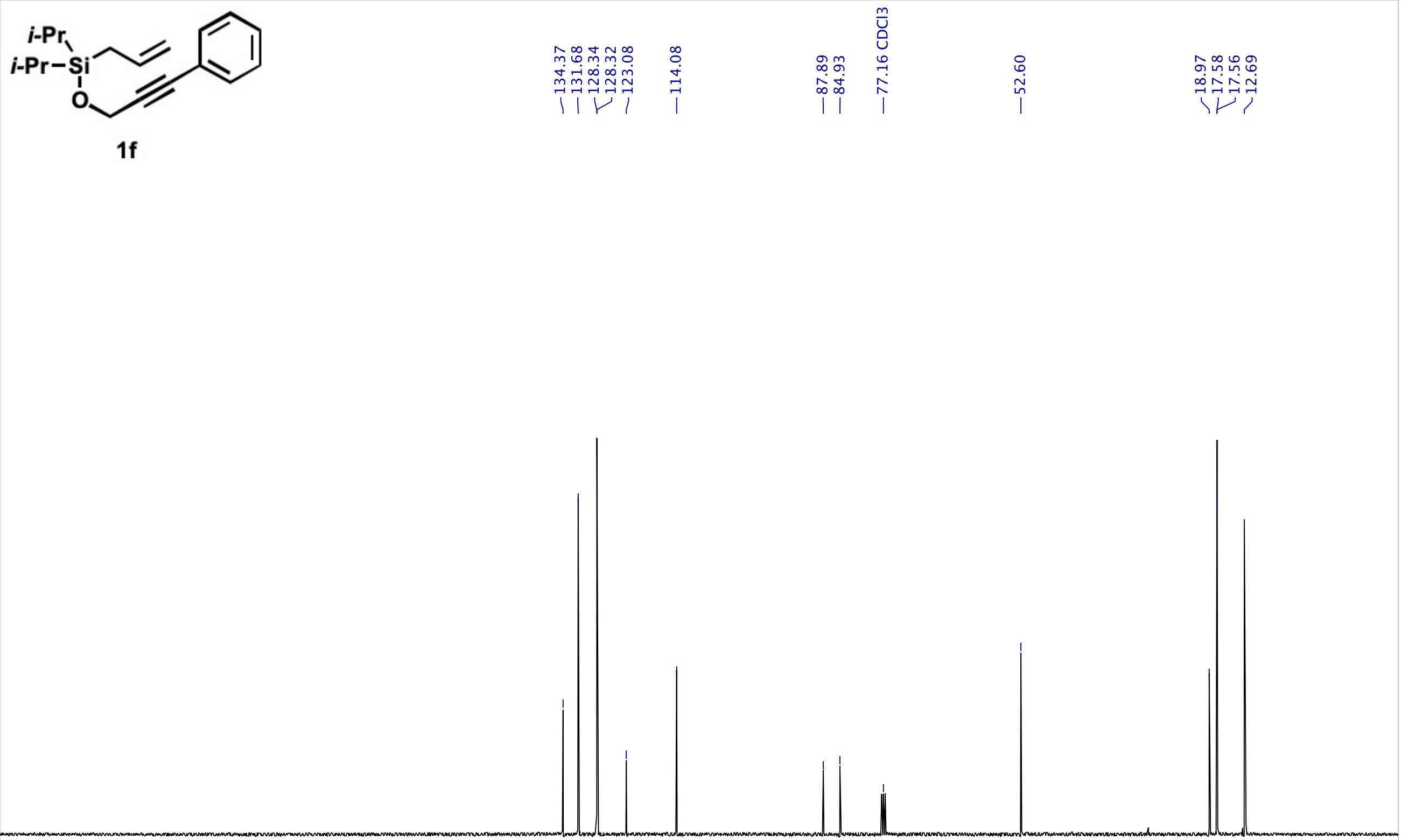


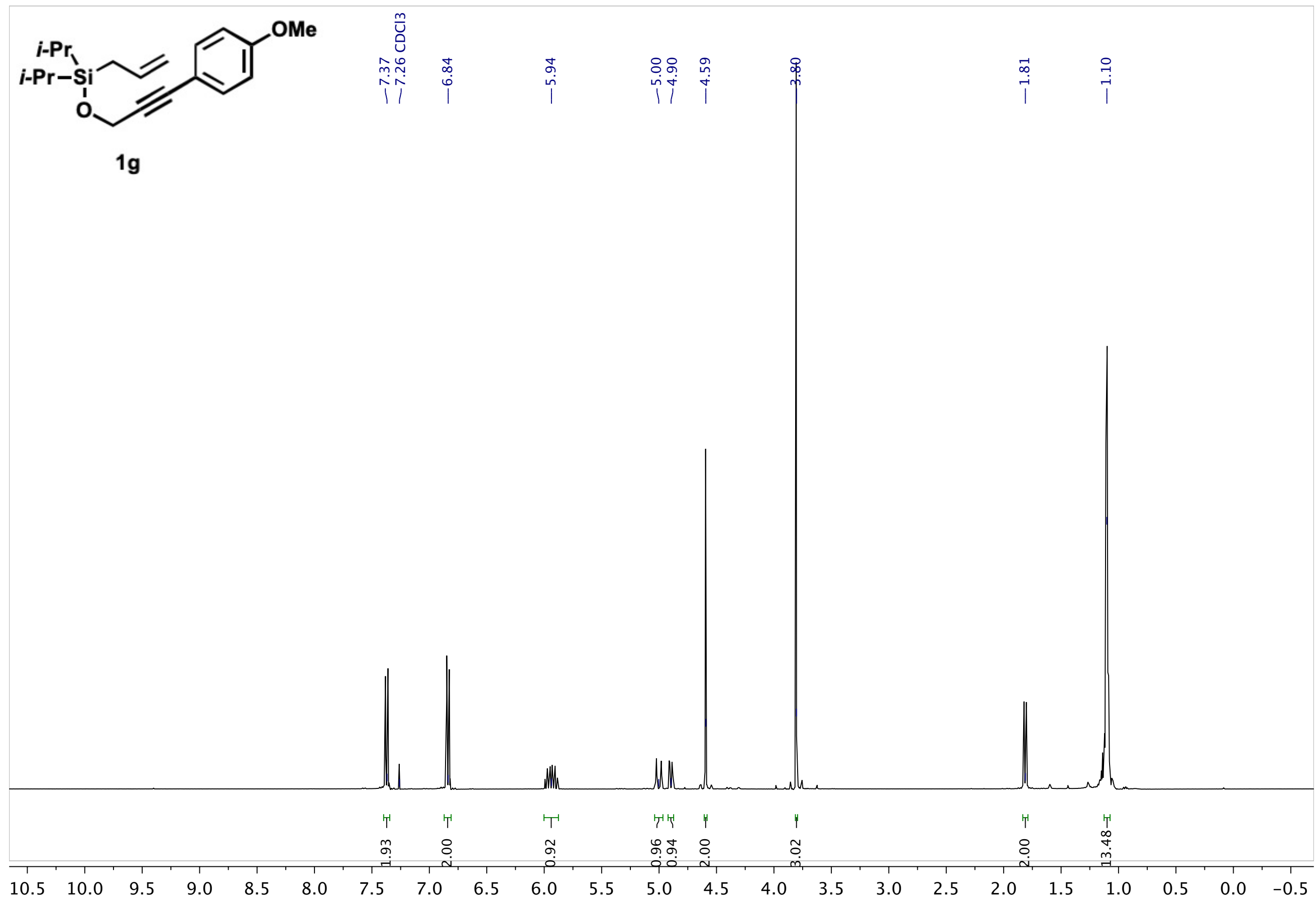



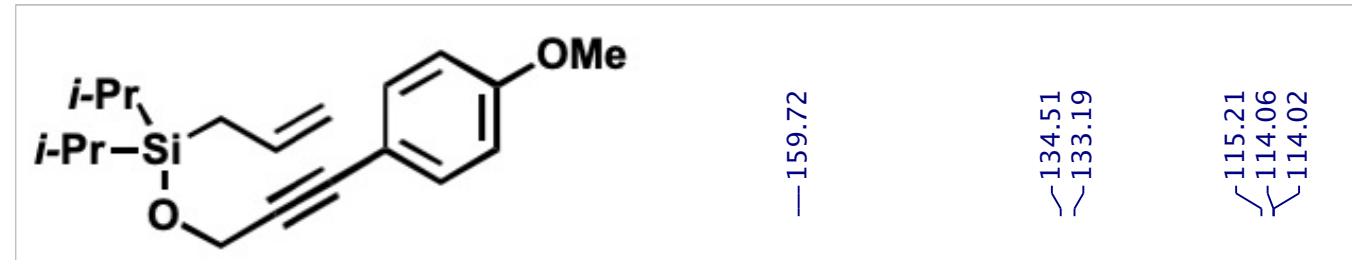

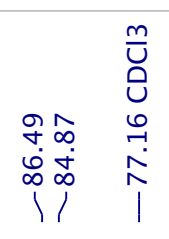

$$
\begin{aligned}
& \text { m욱 } \\
& \text { เท่ก }
\end{aligned}
$$

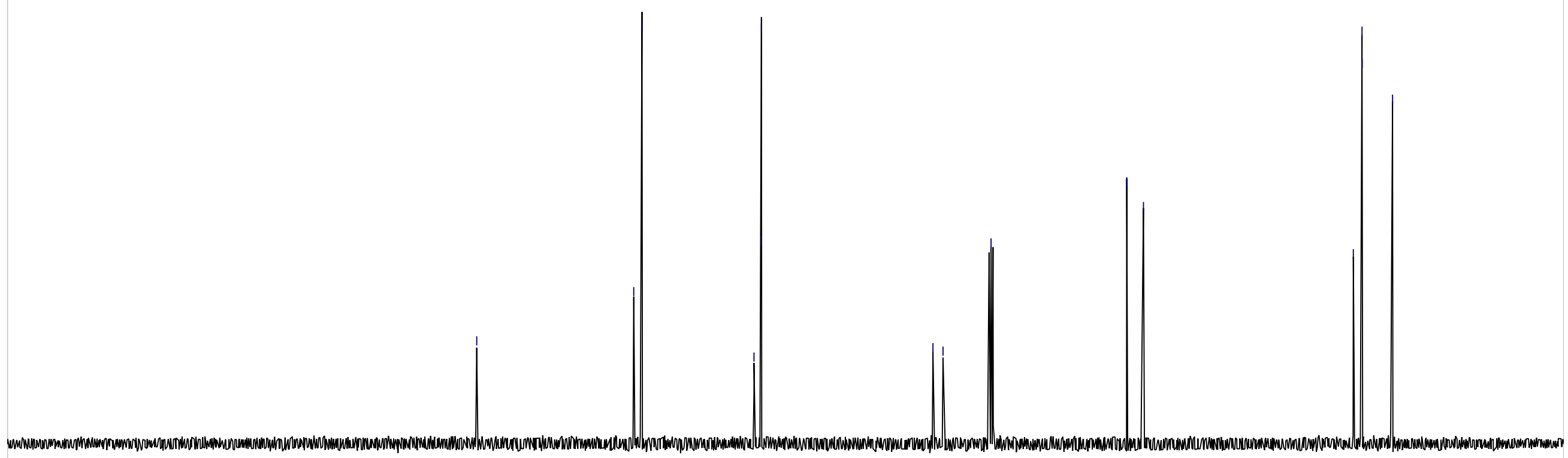



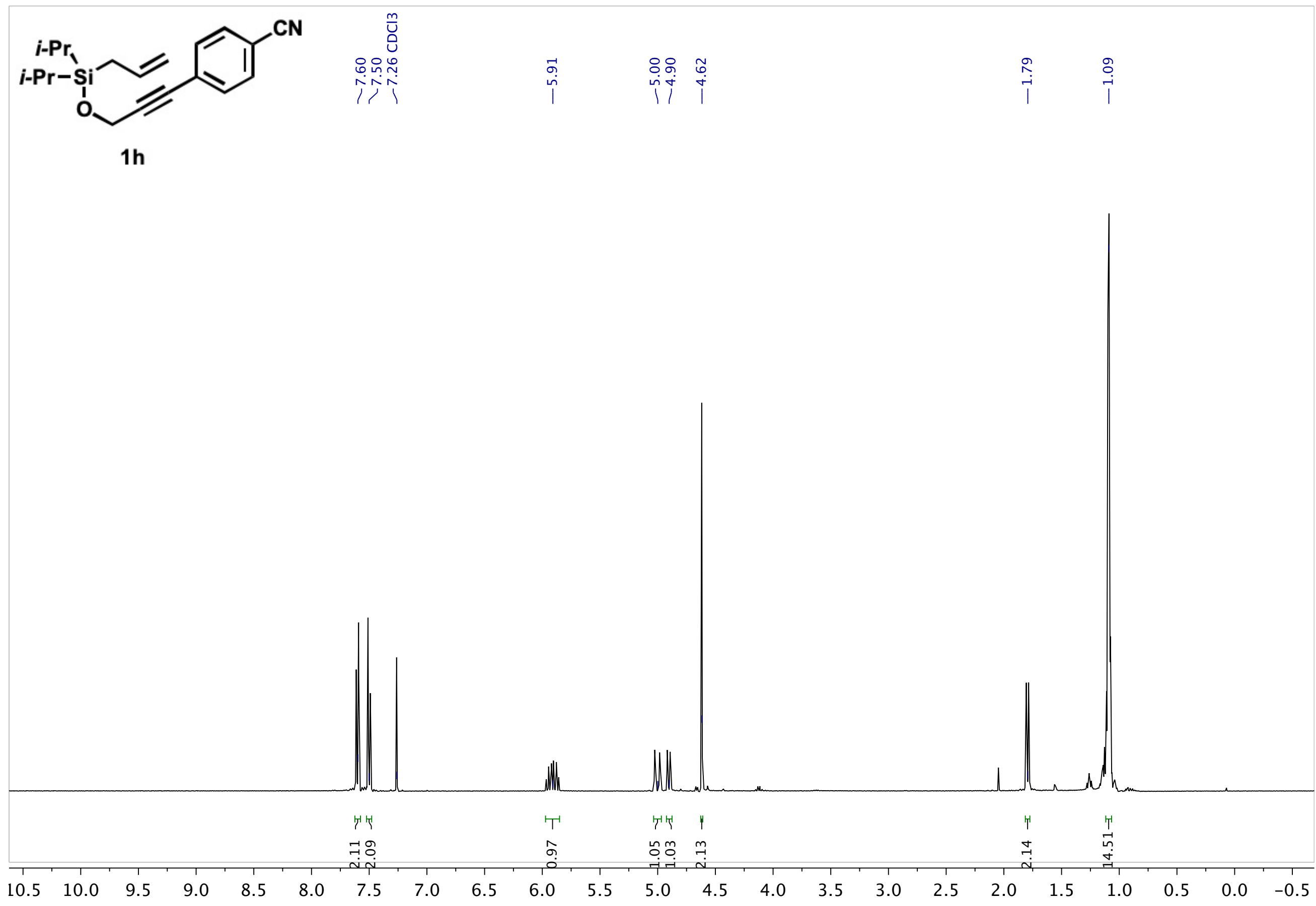


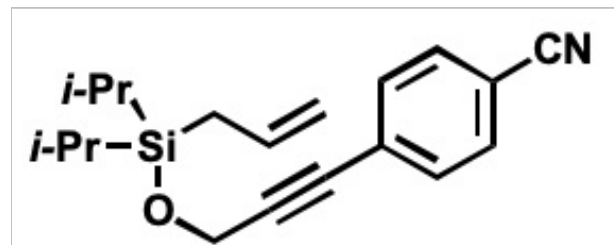

1h

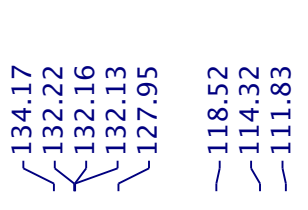

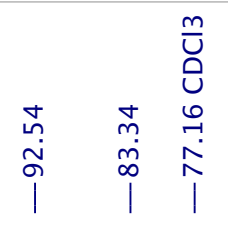

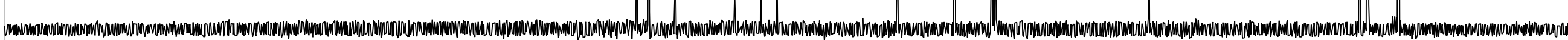

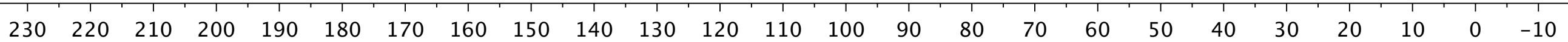




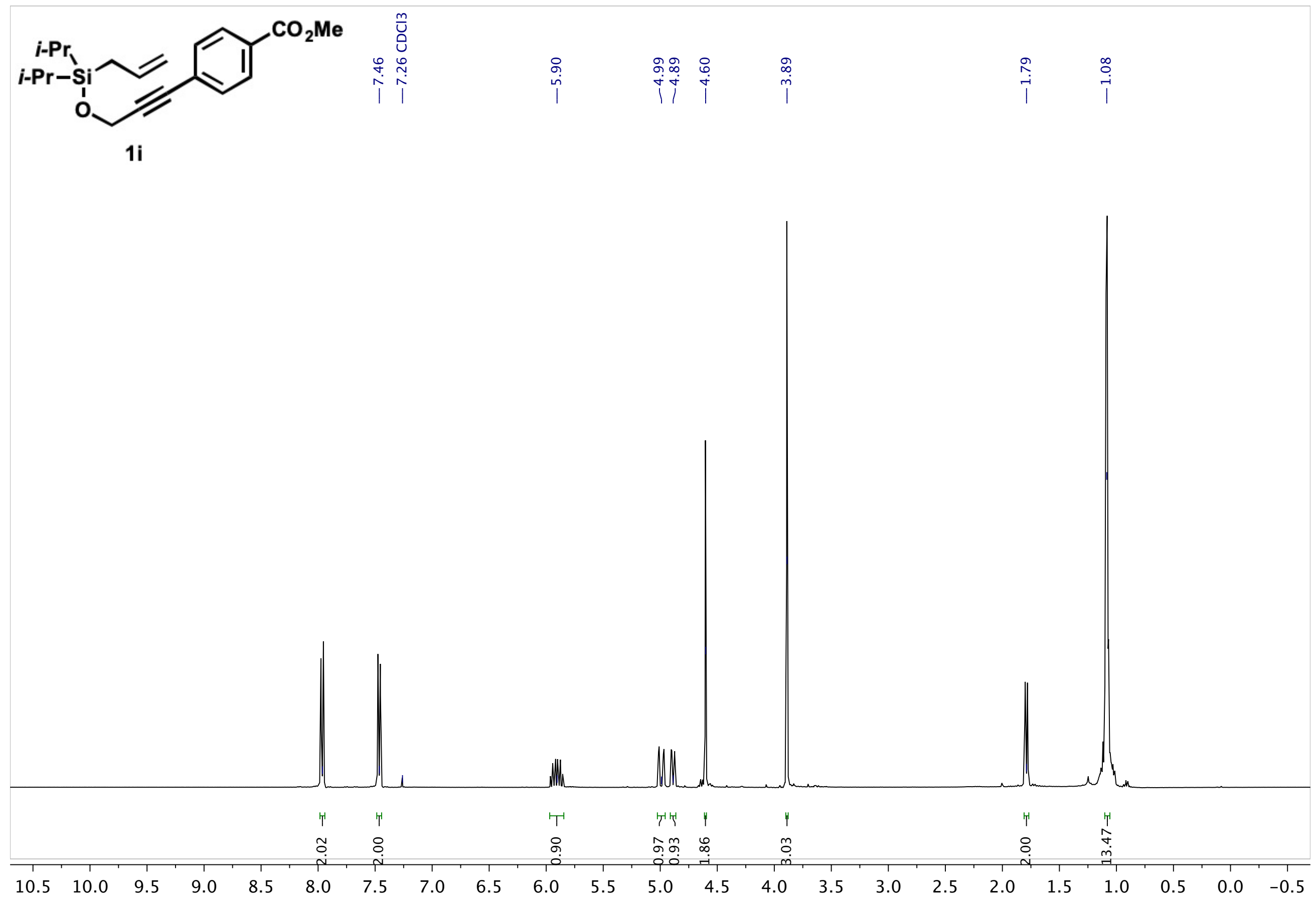



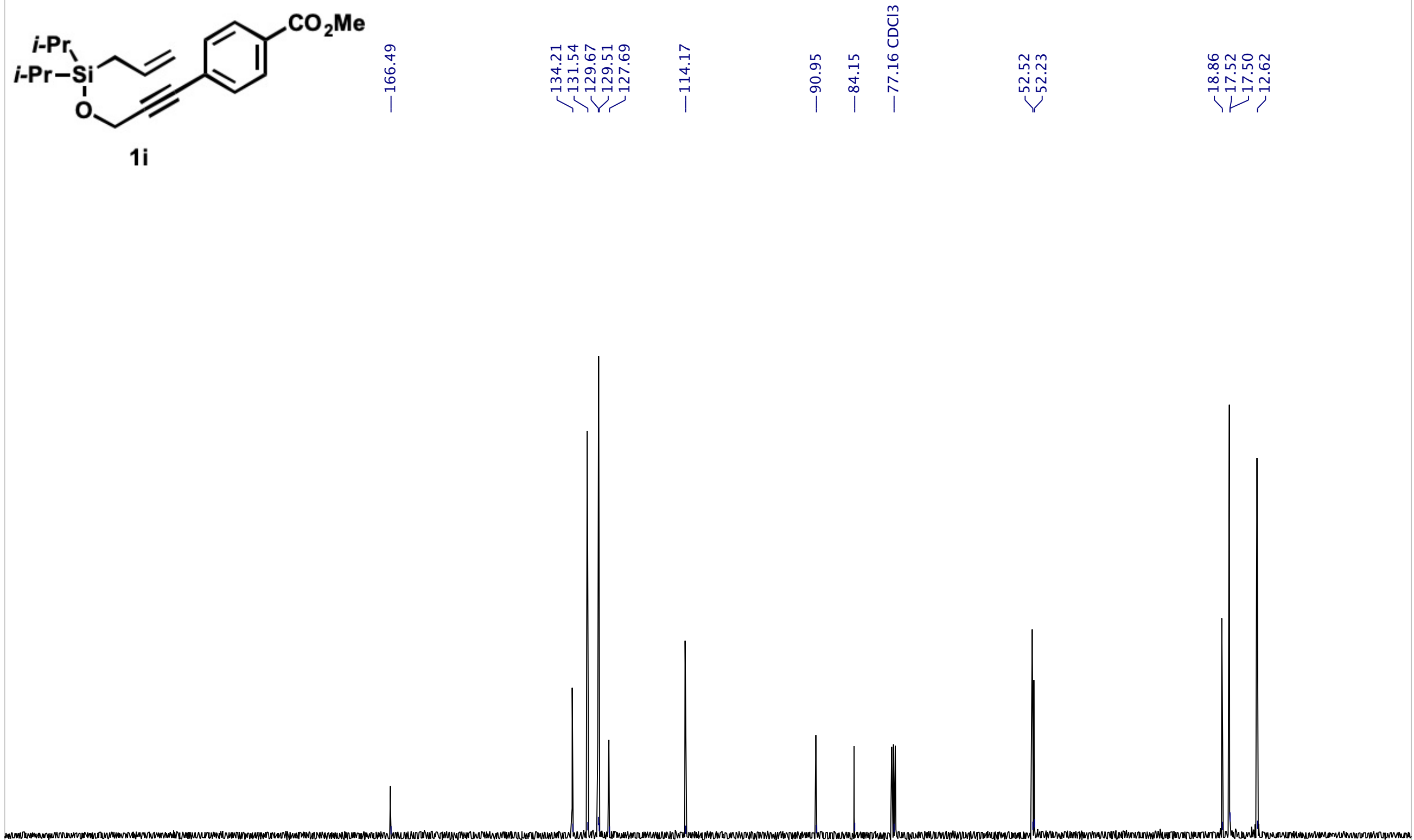


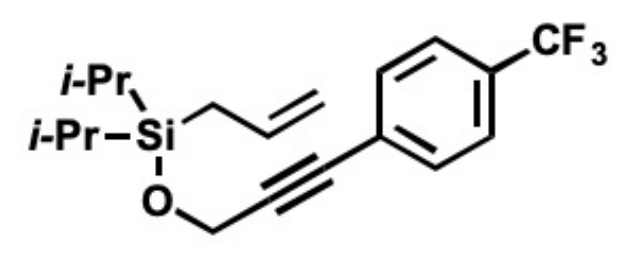

1j

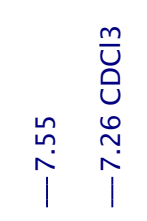

an

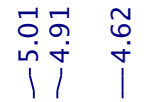

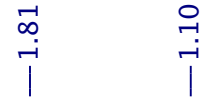

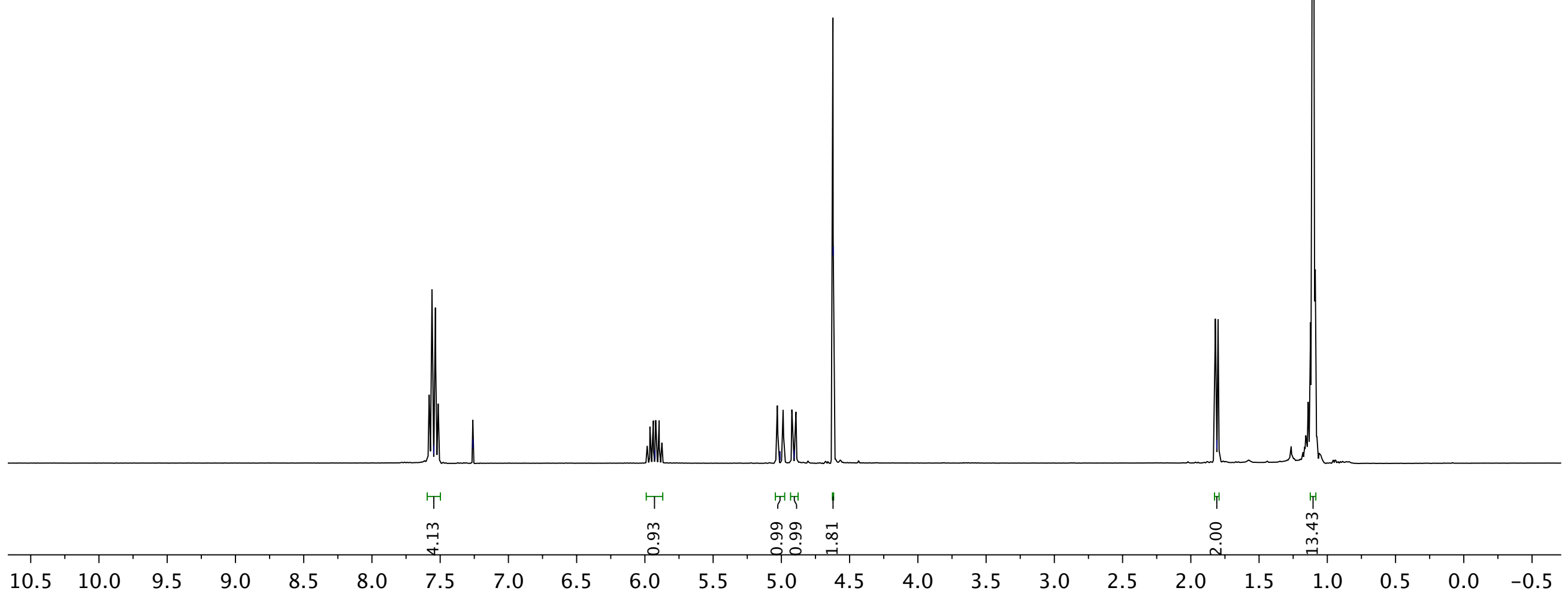




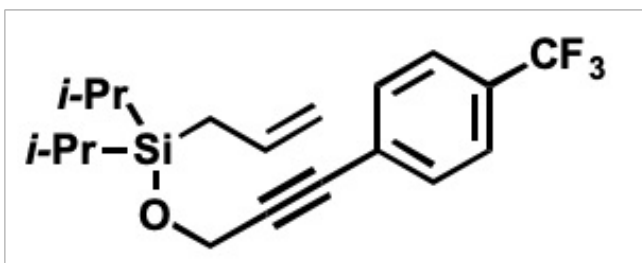

1j
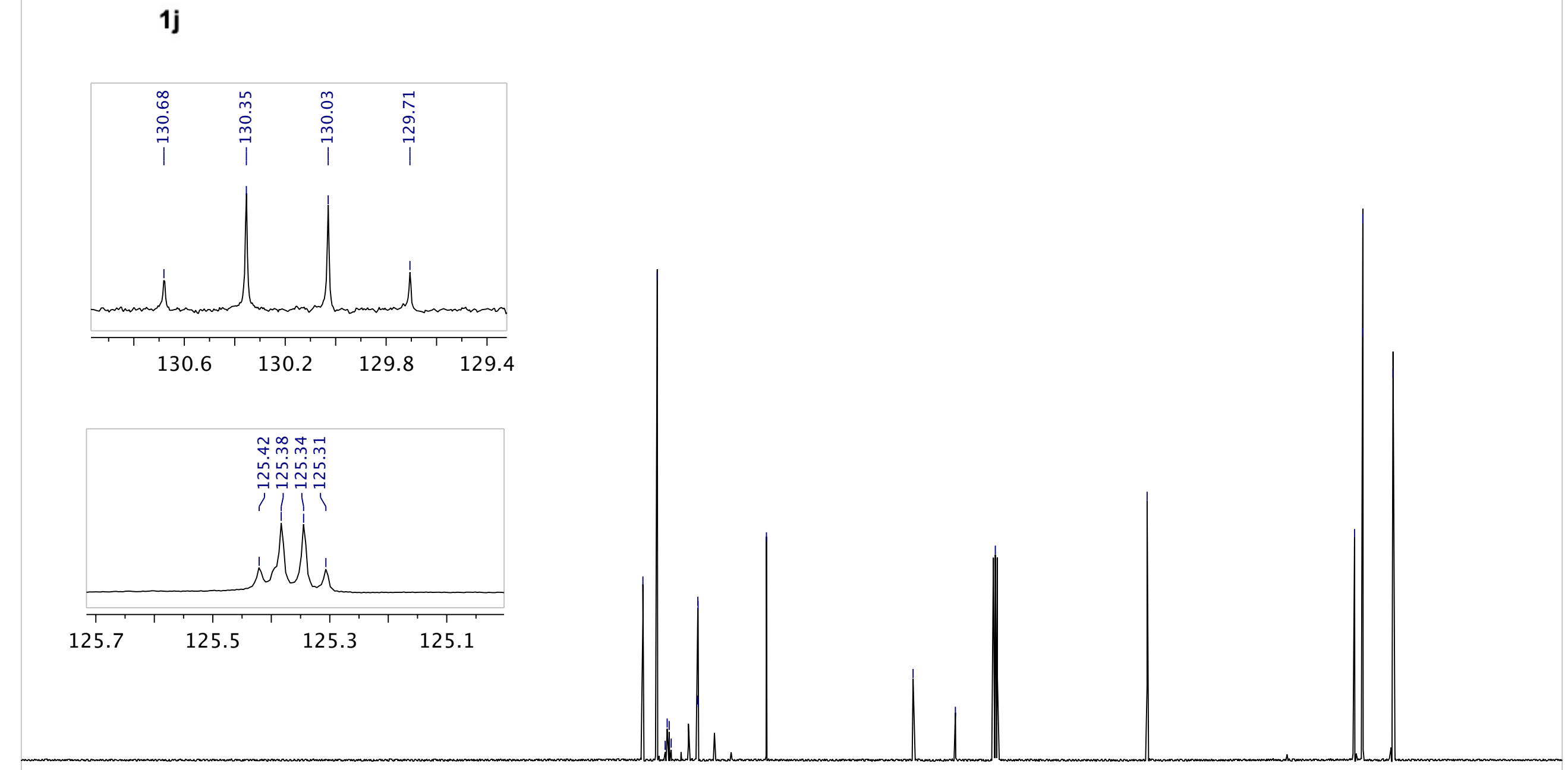

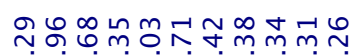

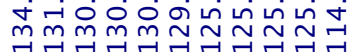
네

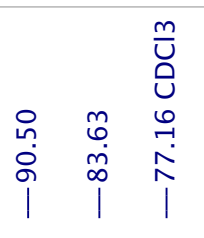

mon

我

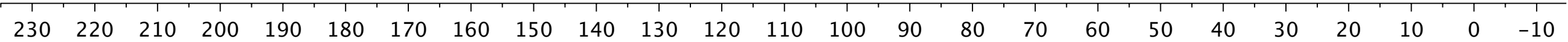



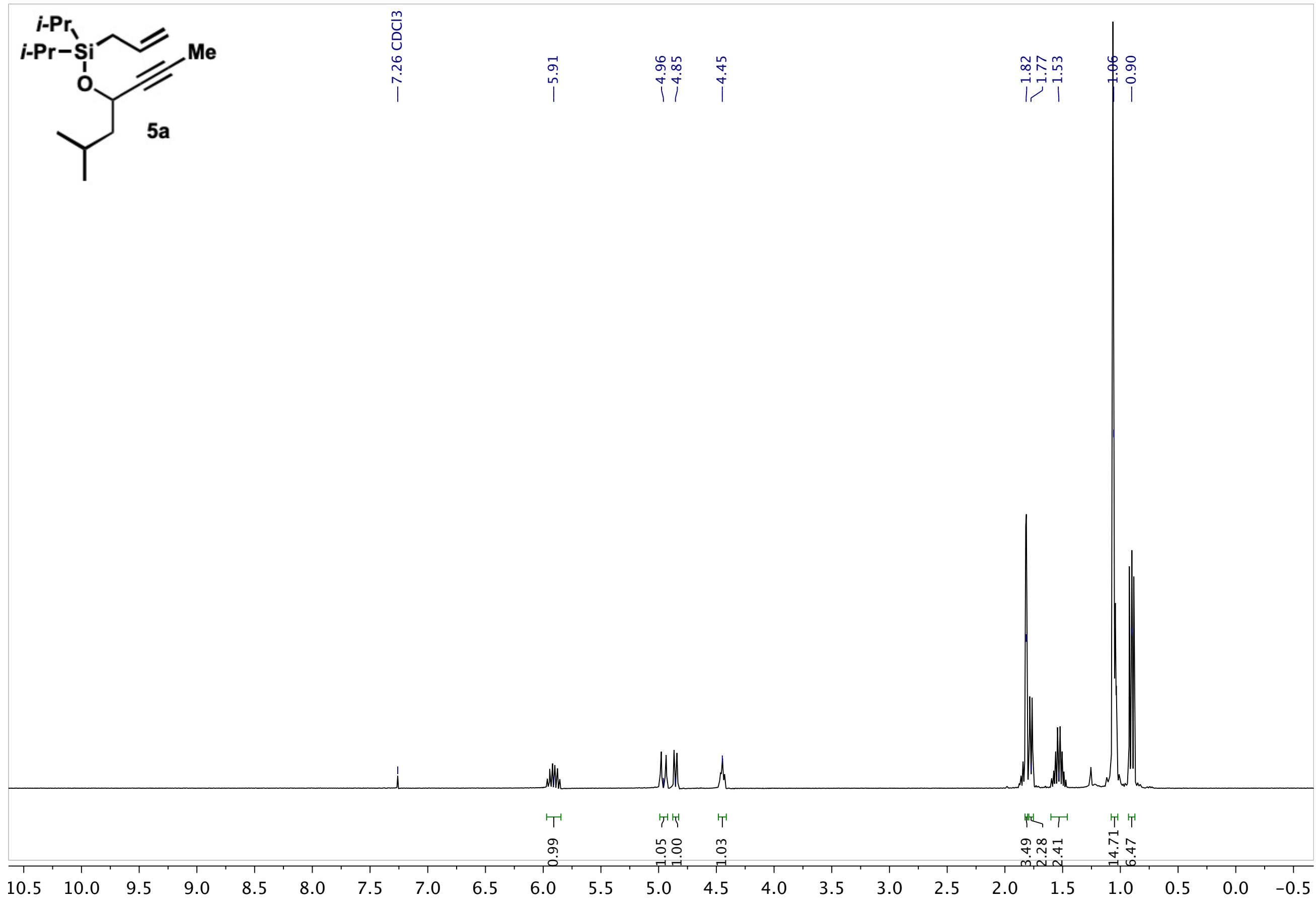


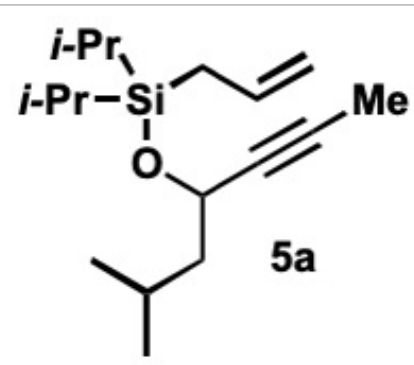

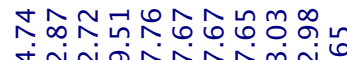

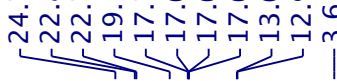

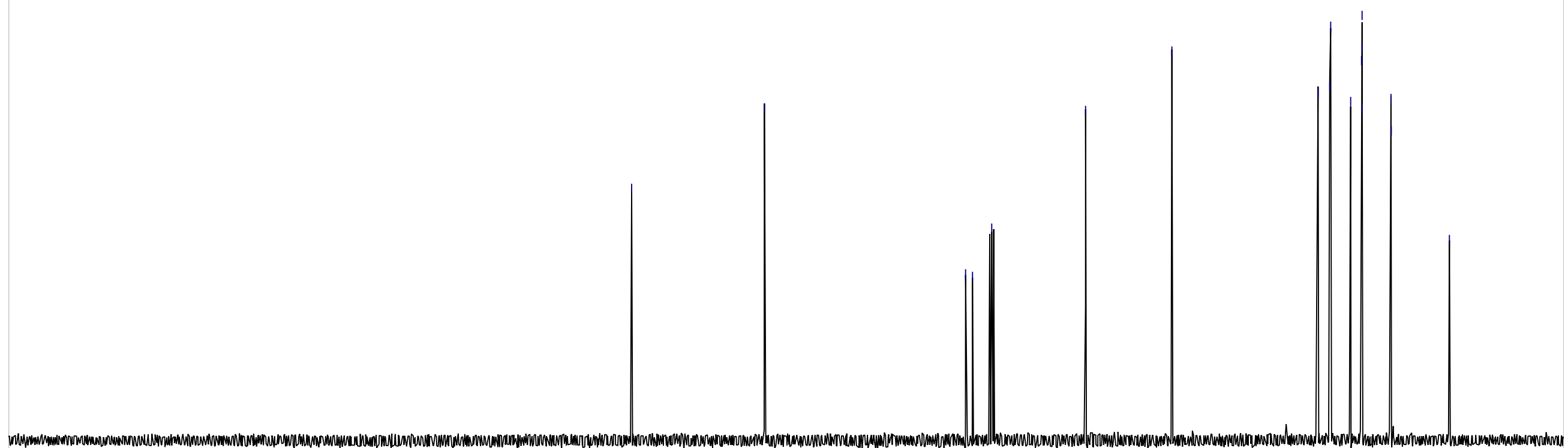


S65

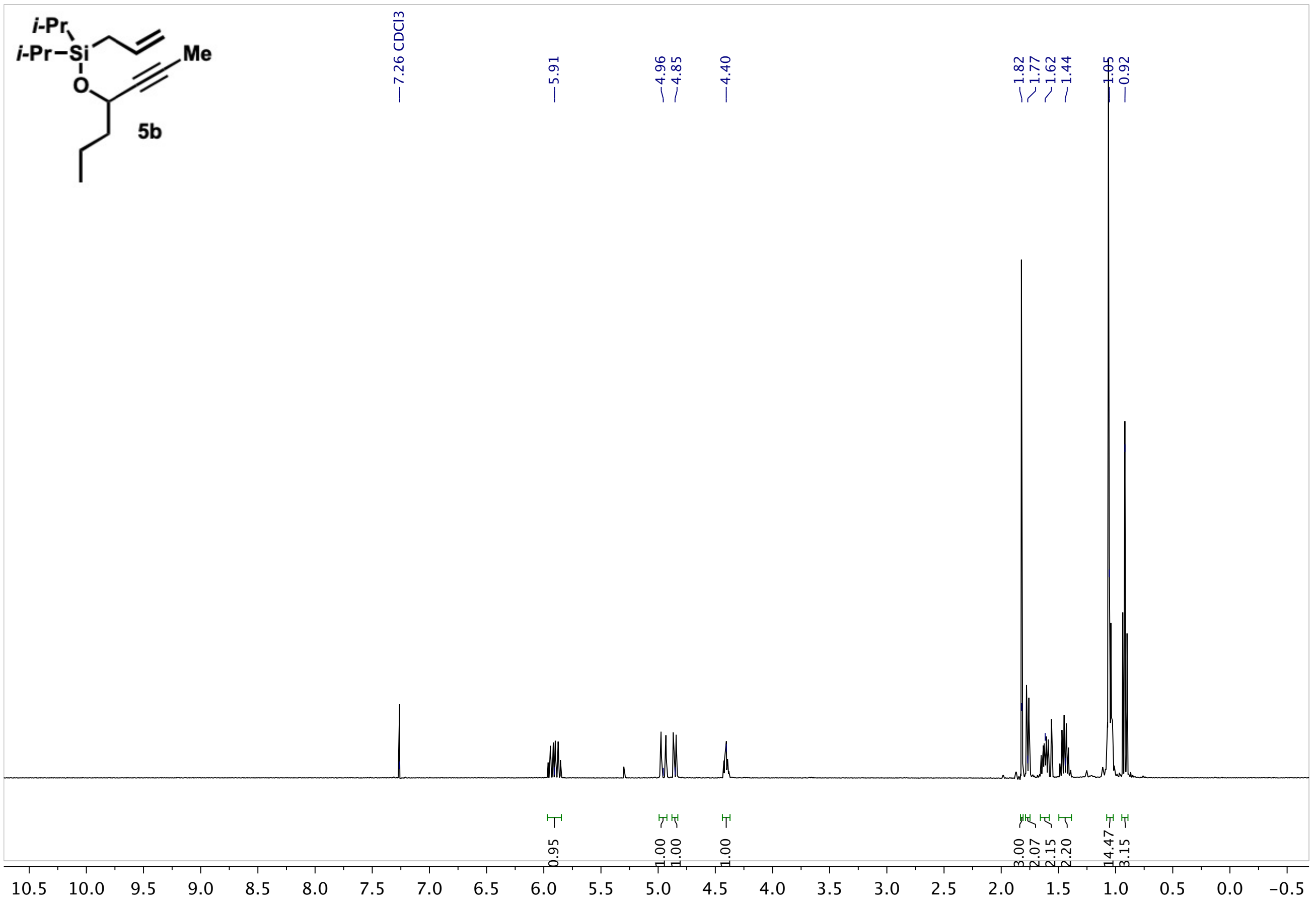


S66<smiles>C=CC[Si](CCC)(OC(C#CC)CCC)P([In])C(C)C</smiles>
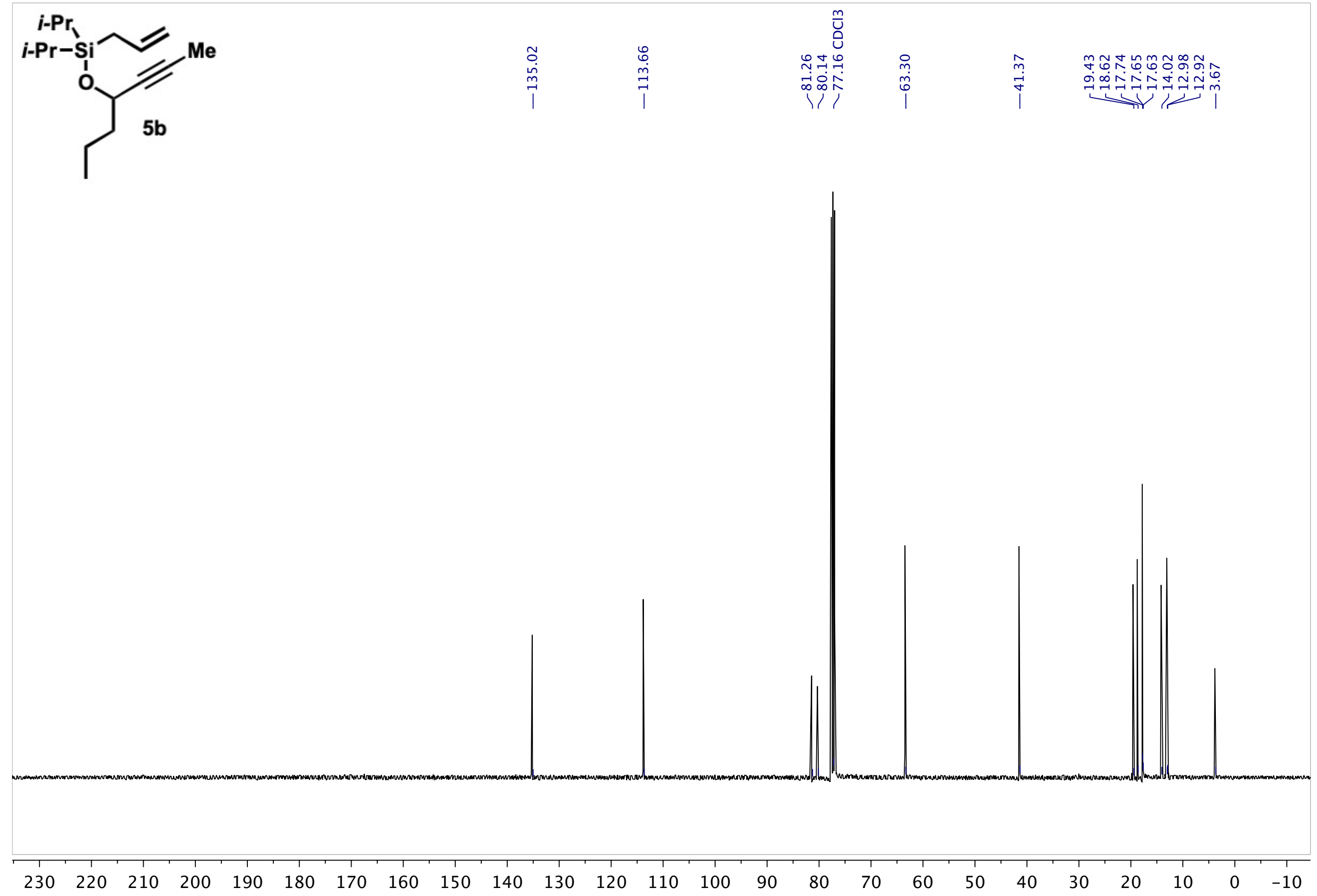


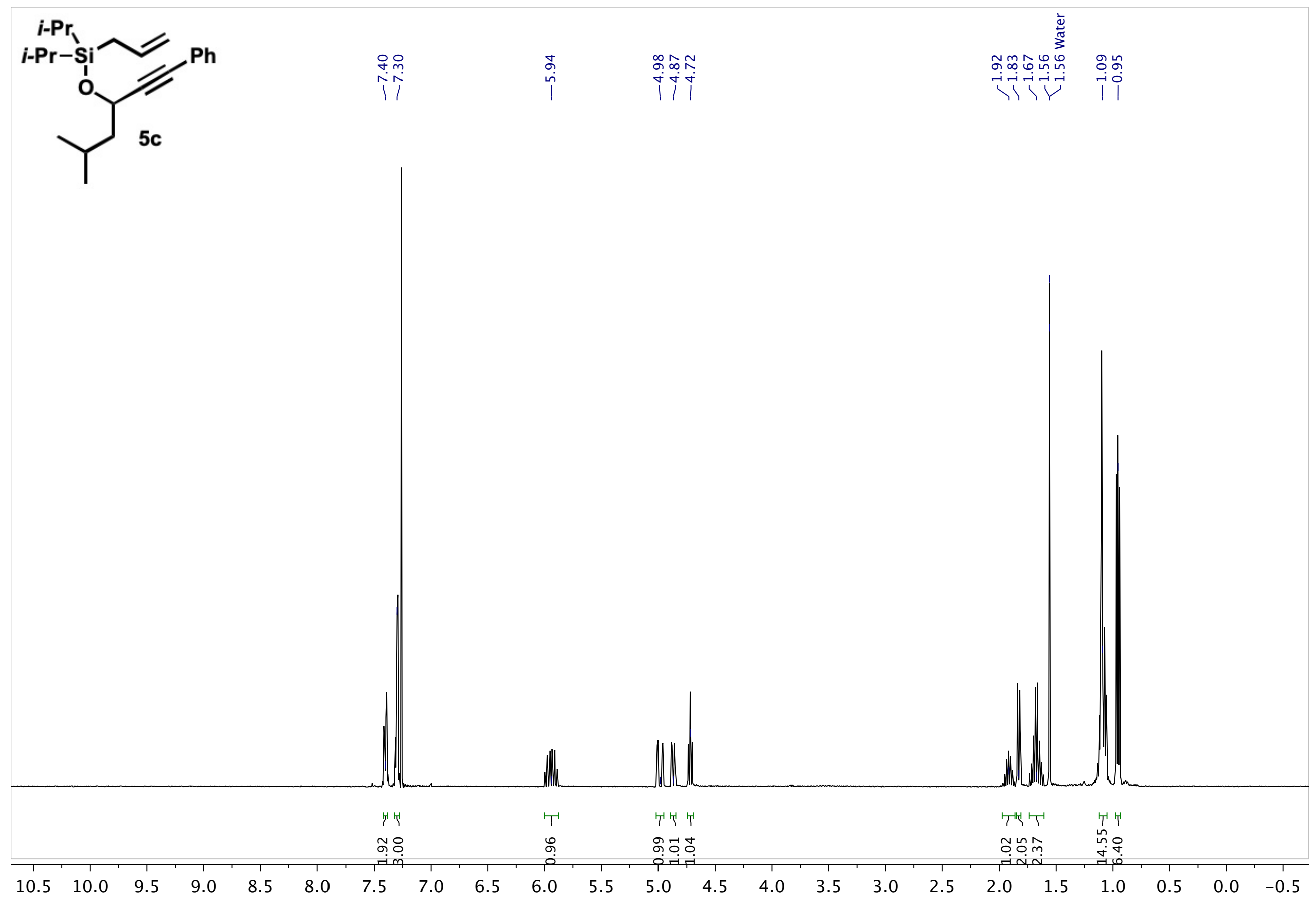




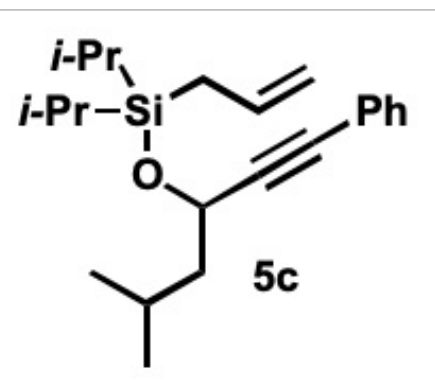

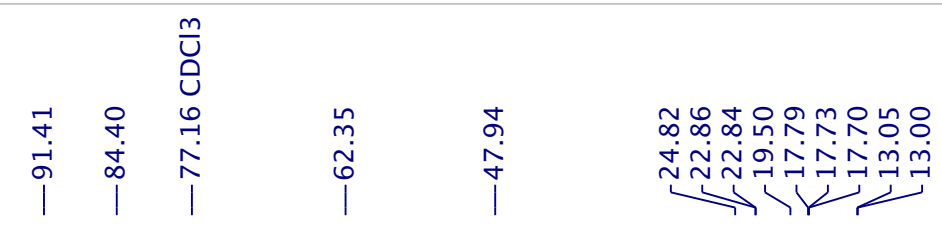

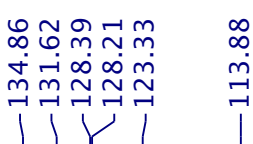

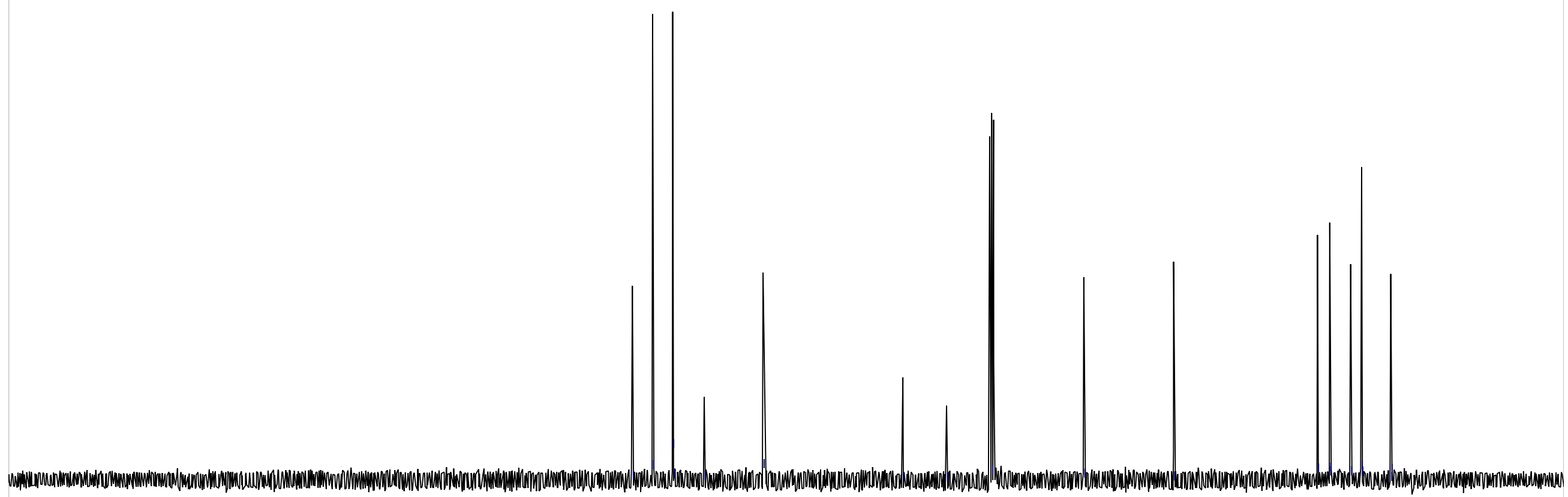




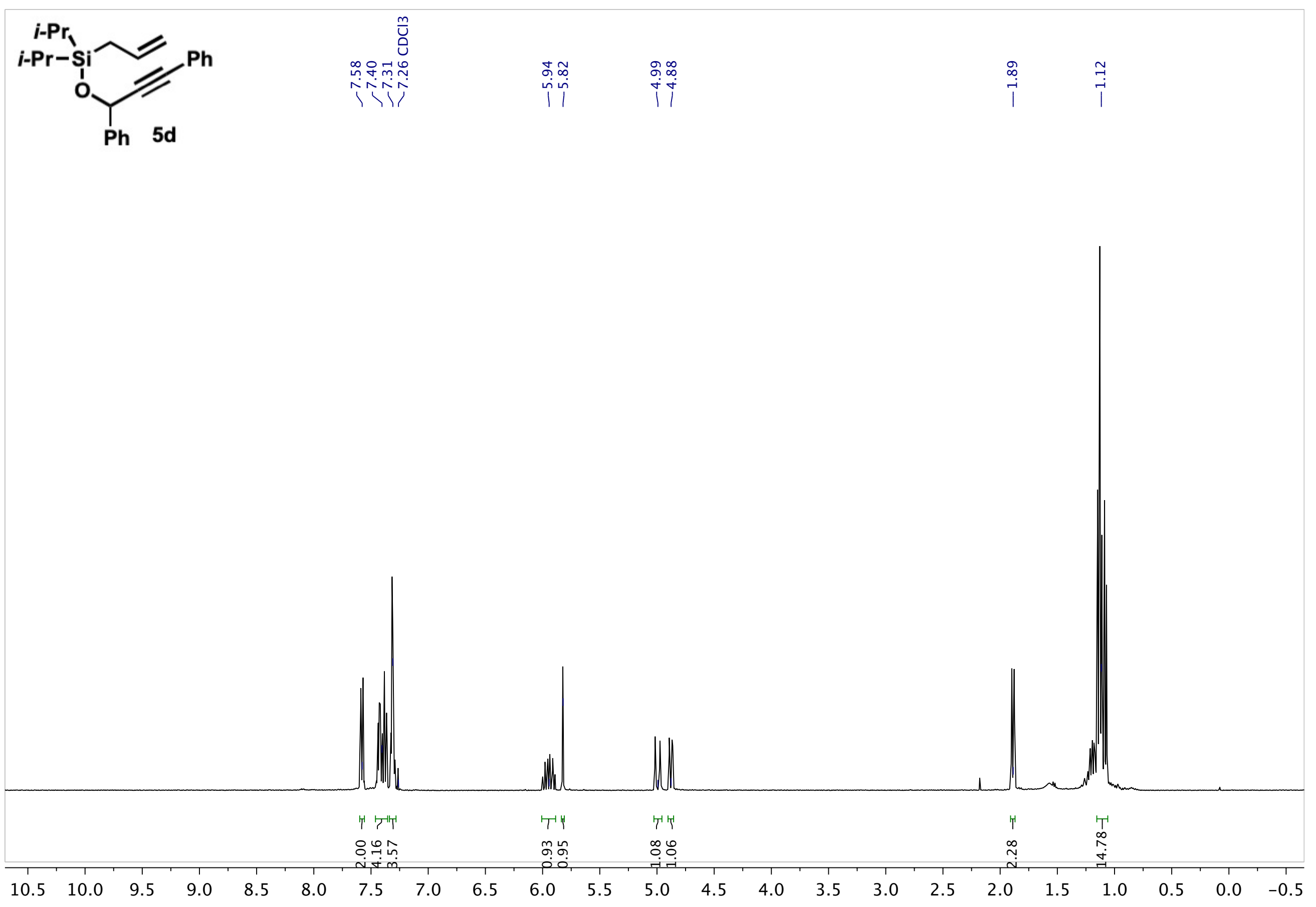


<smiles>C=CC[Si](CCC)(OC(C#CPc1ccccc1)c1ccccc1)C(C)C</smiles>

Ph 5d

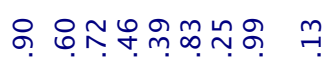

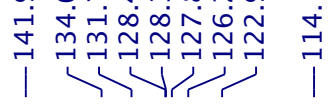

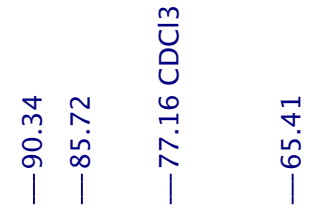
ㄴํㅅNㅇㅇㅇำ จำกี่ง่า

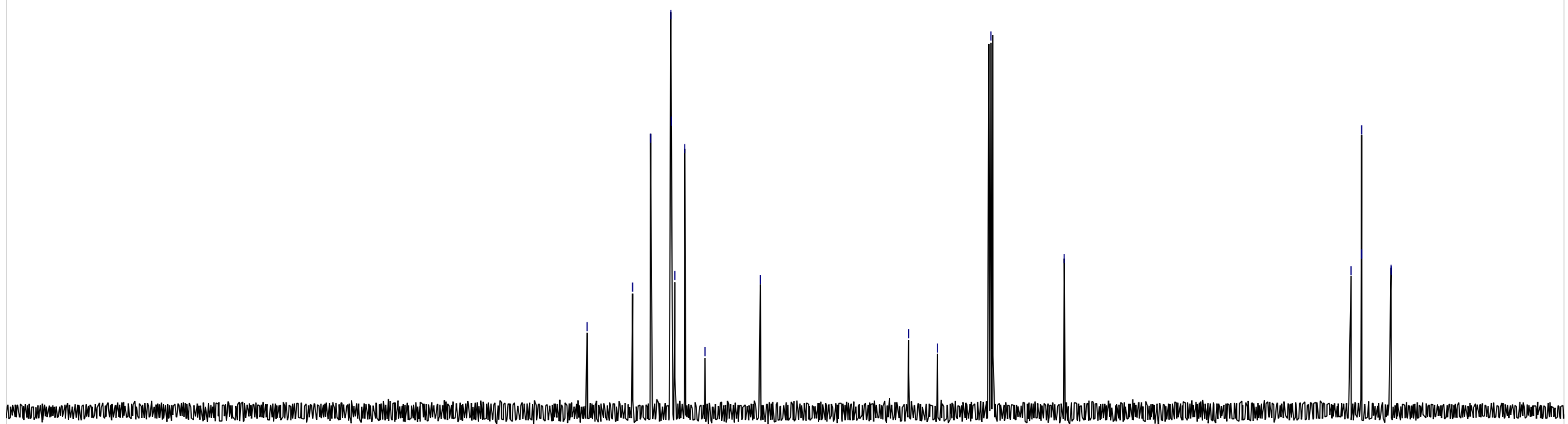




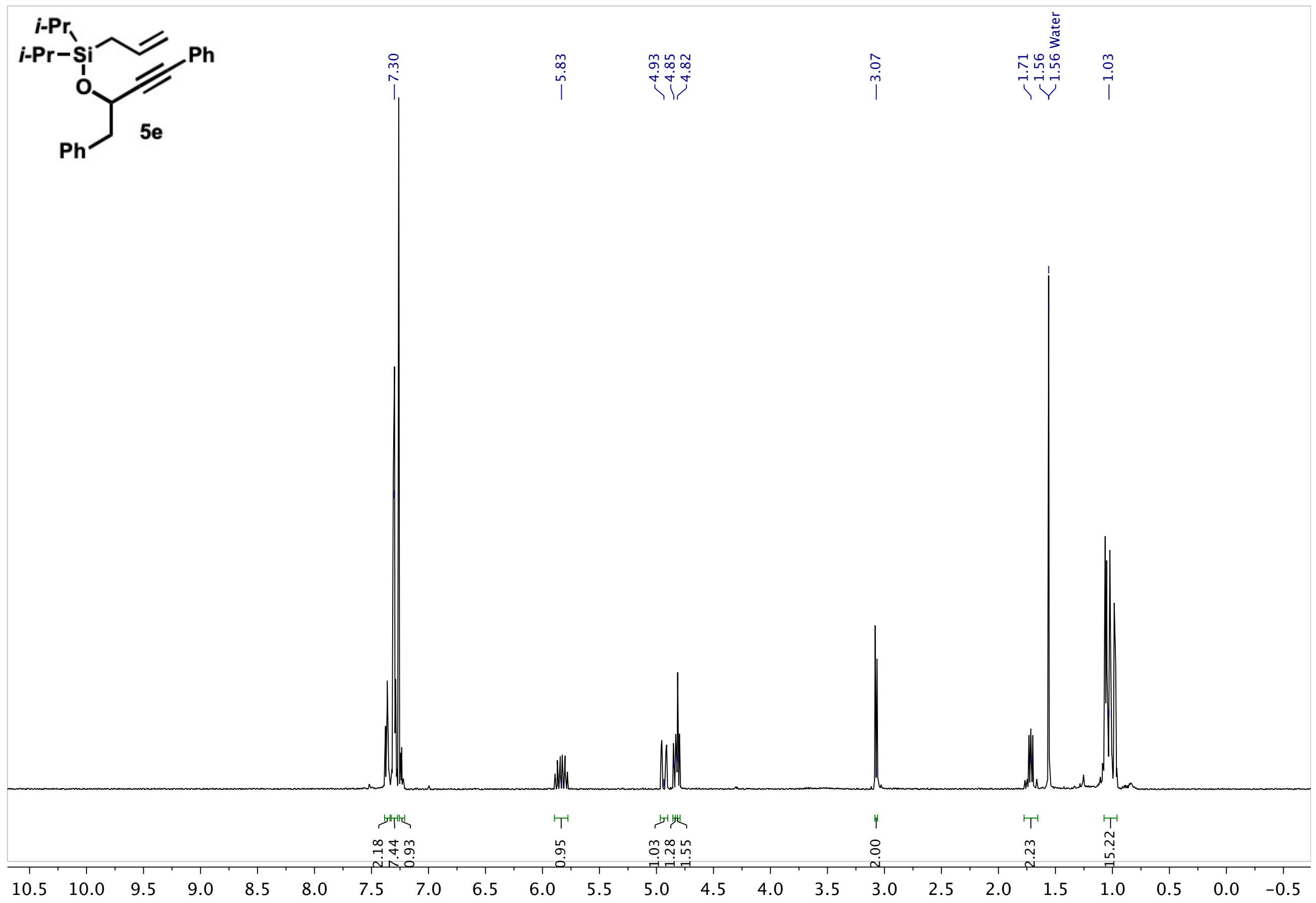




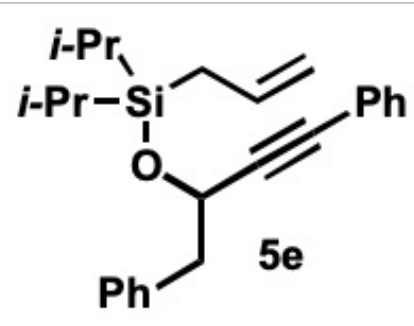

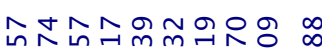

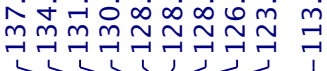

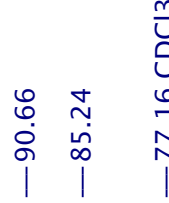

$m$
$\underline{U}$
0
0
0
$i$
$i$
$i$

$\overrightarrow{0}$
$\dot{0}$
1

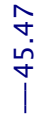

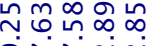

玲

(n)

(

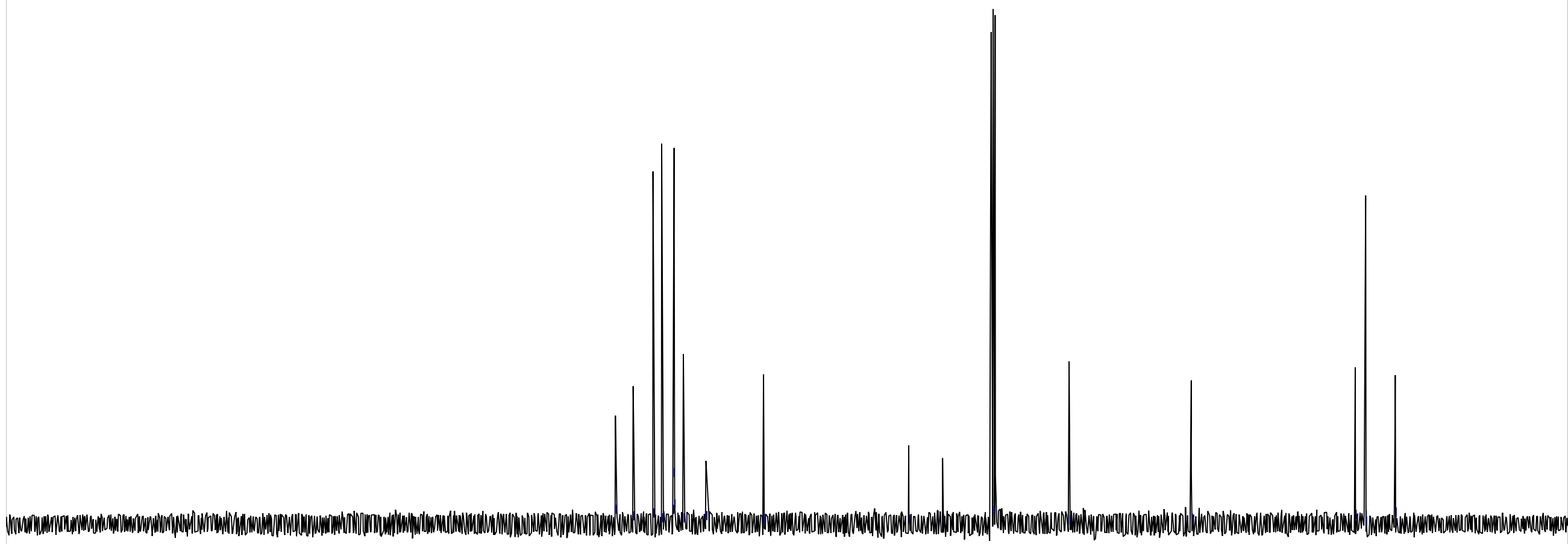




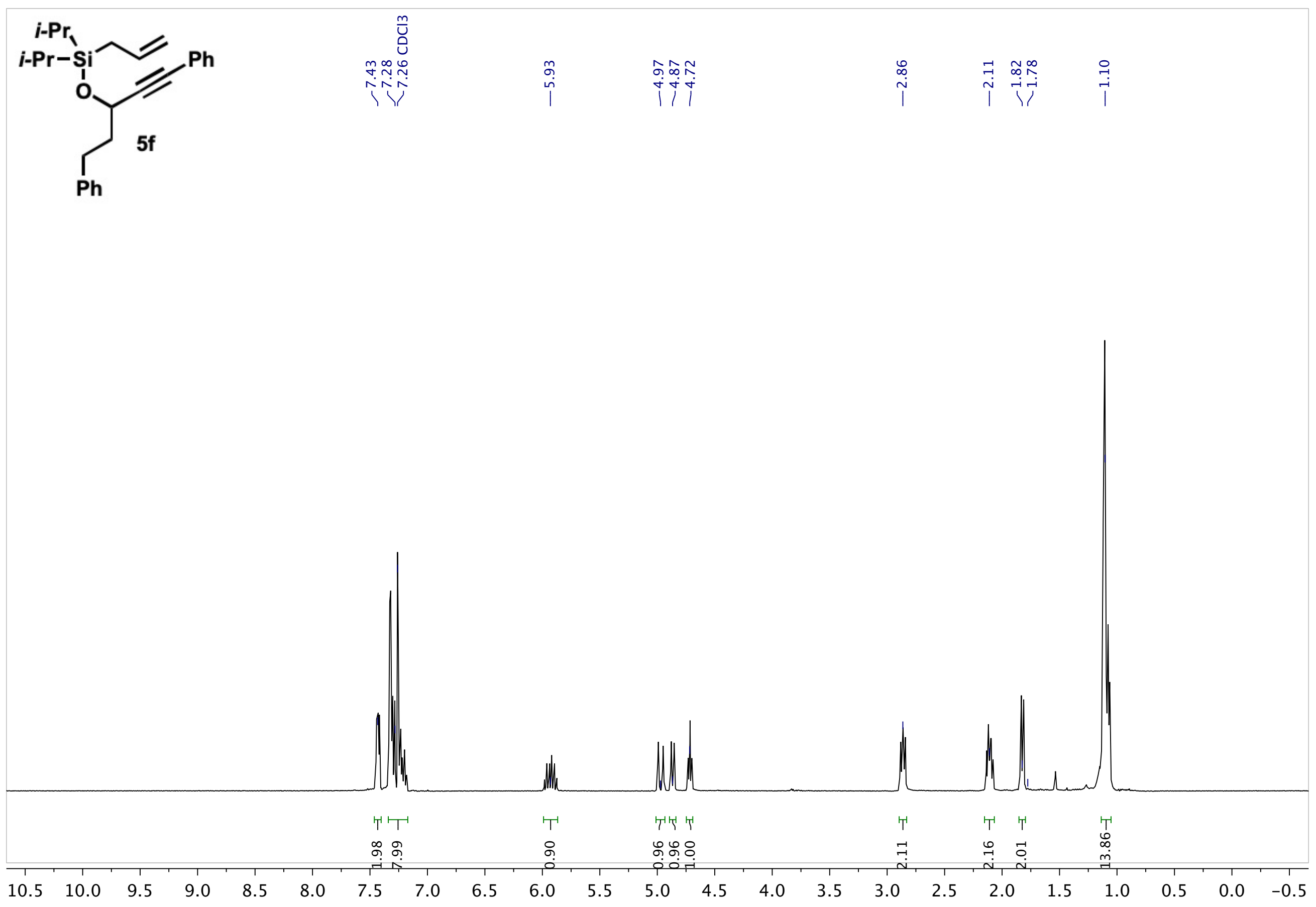




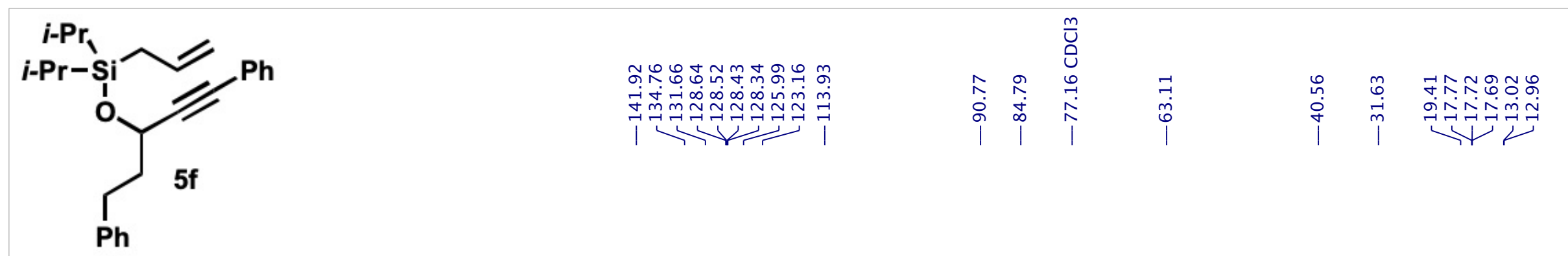

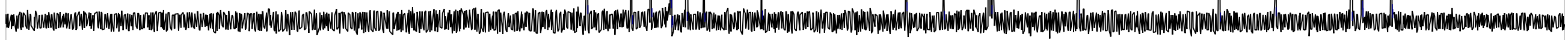

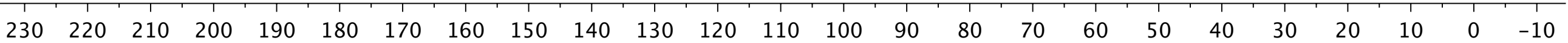



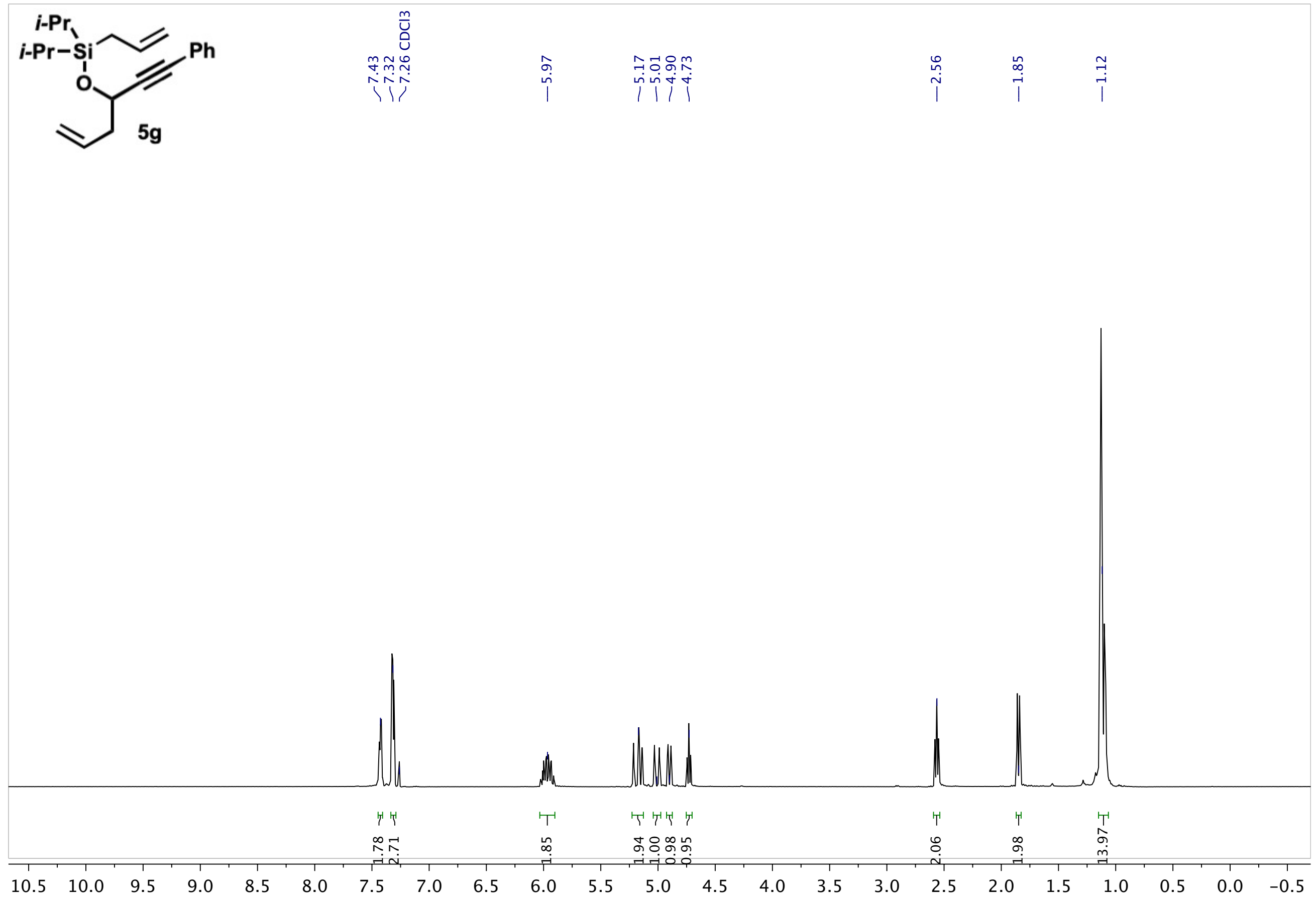


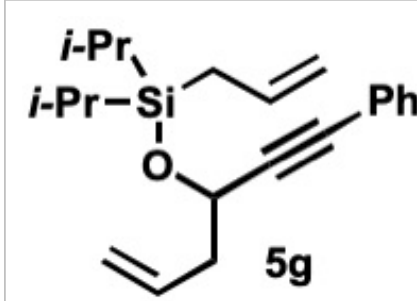

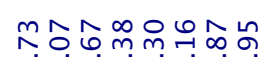

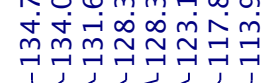

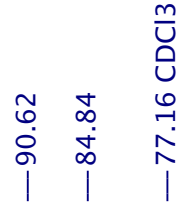

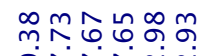

จำ่ำ่ส

5

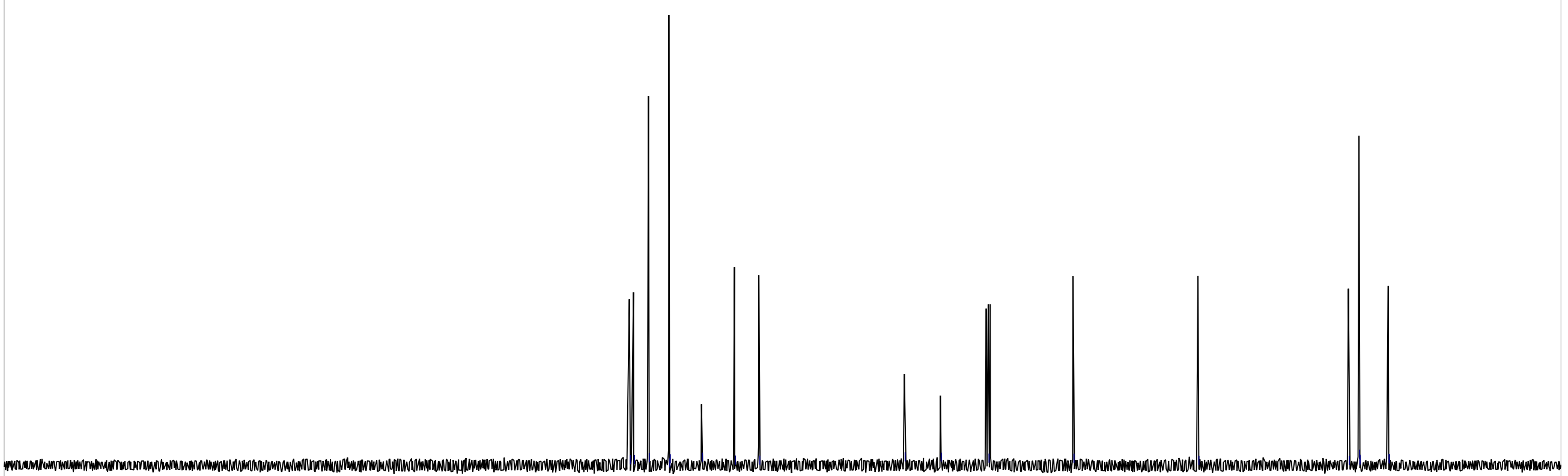

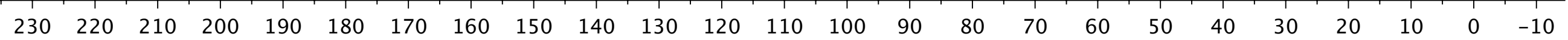




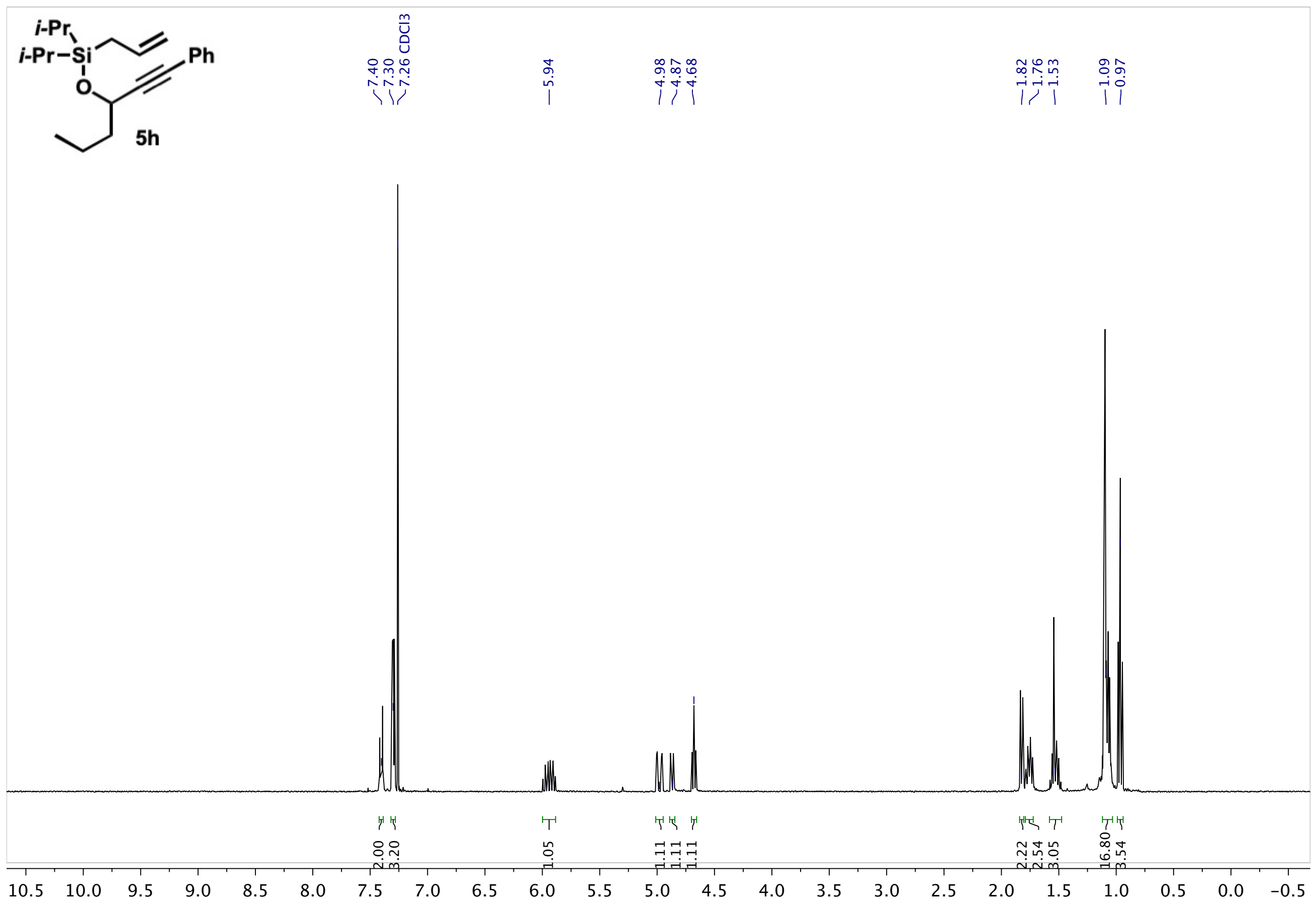




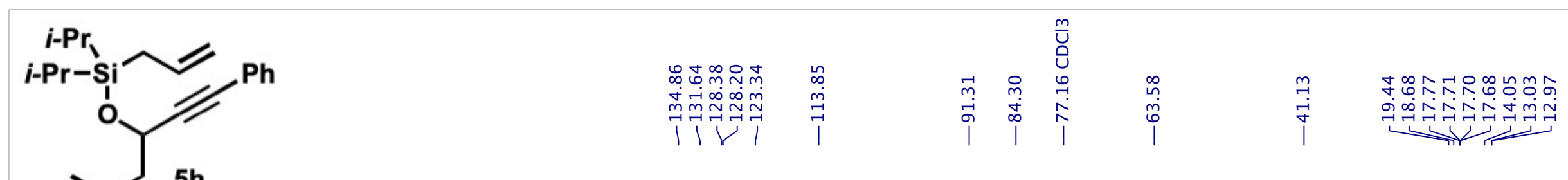

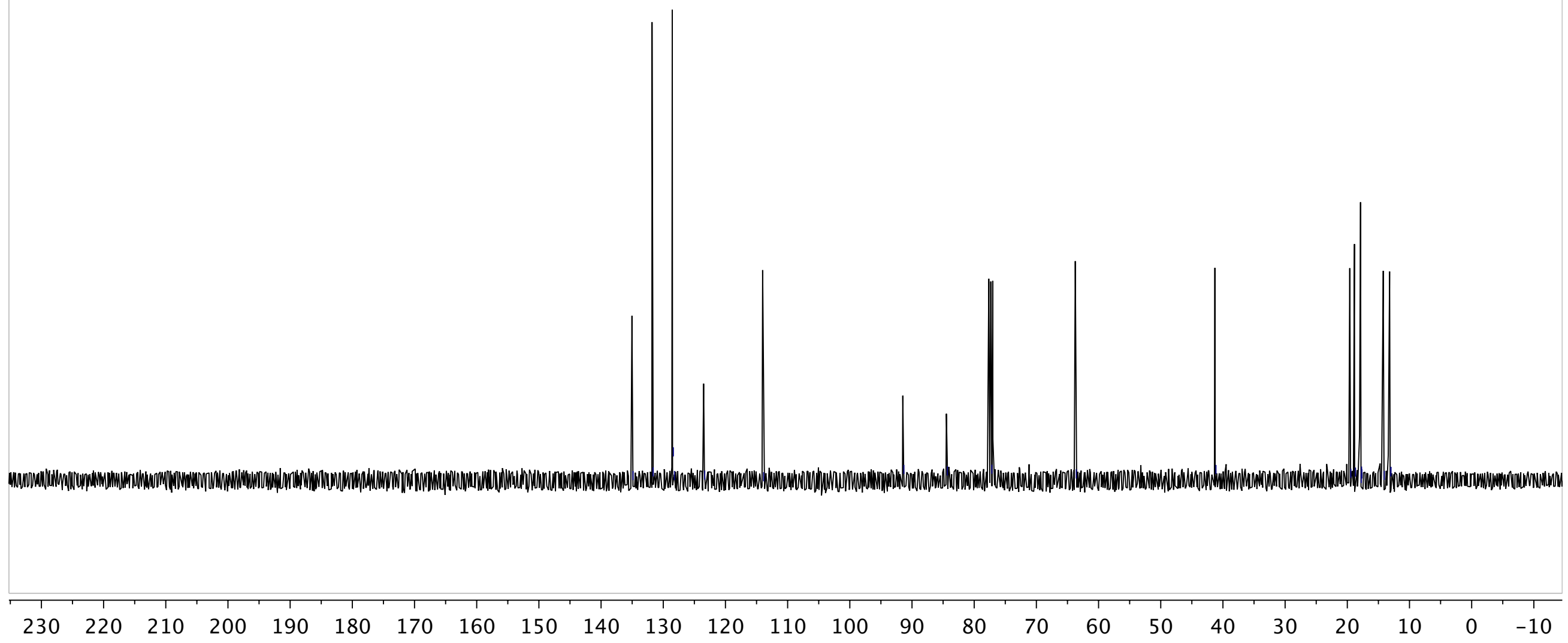



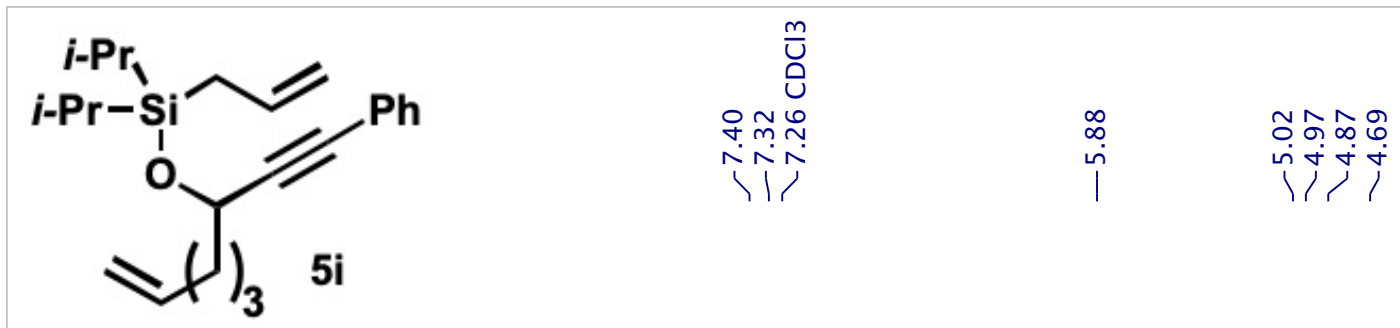

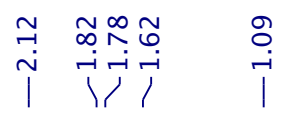

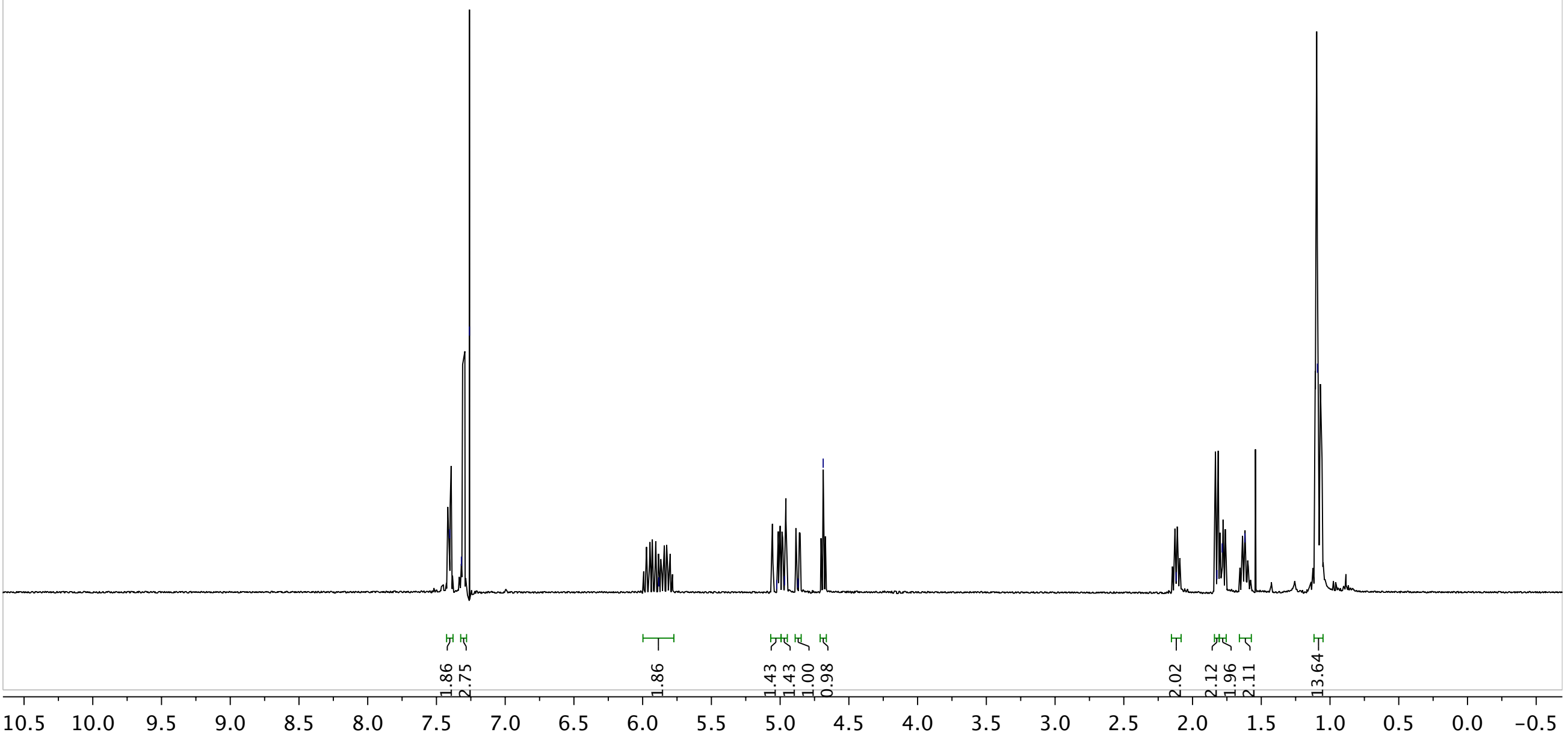



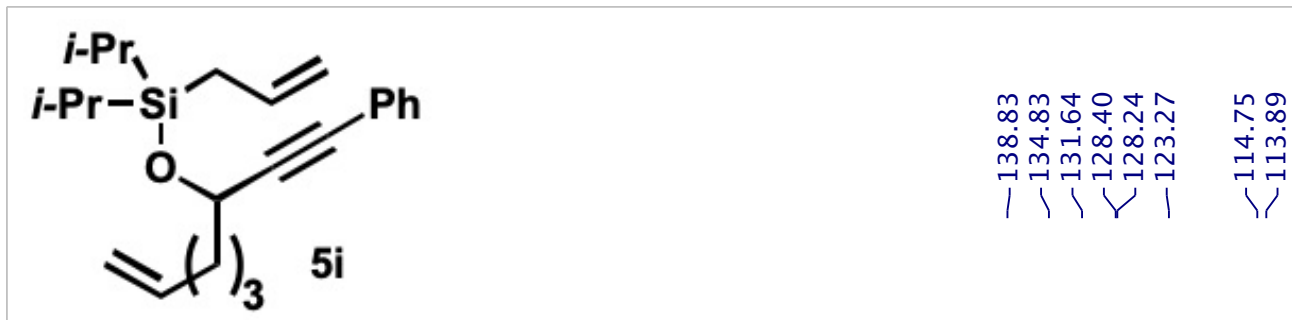

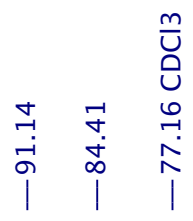

하

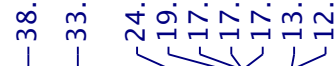

3

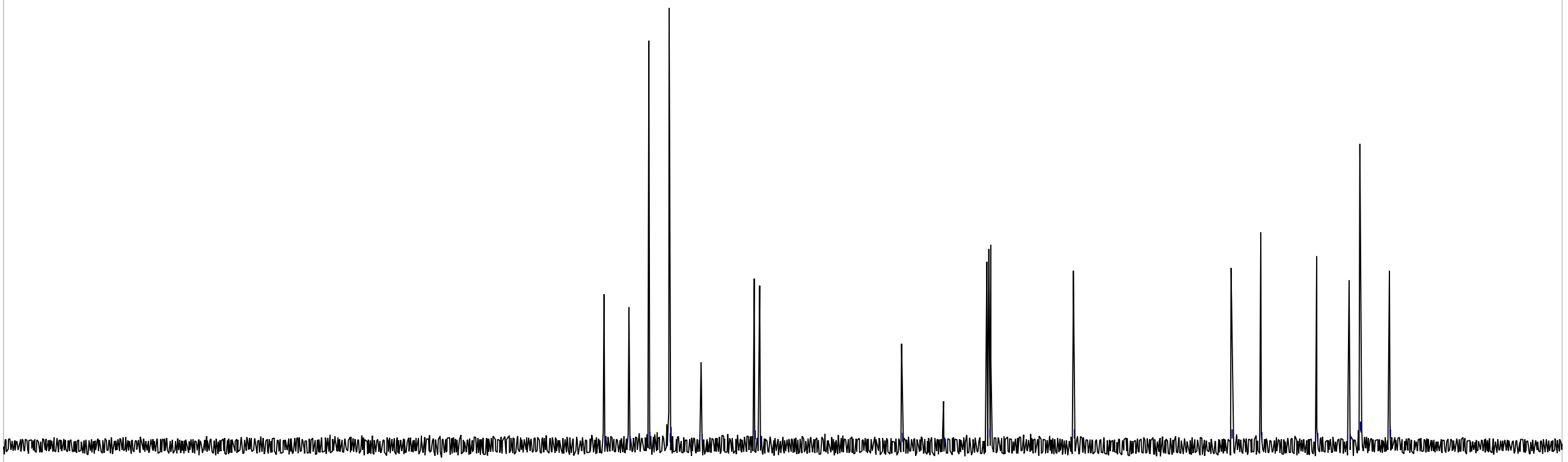



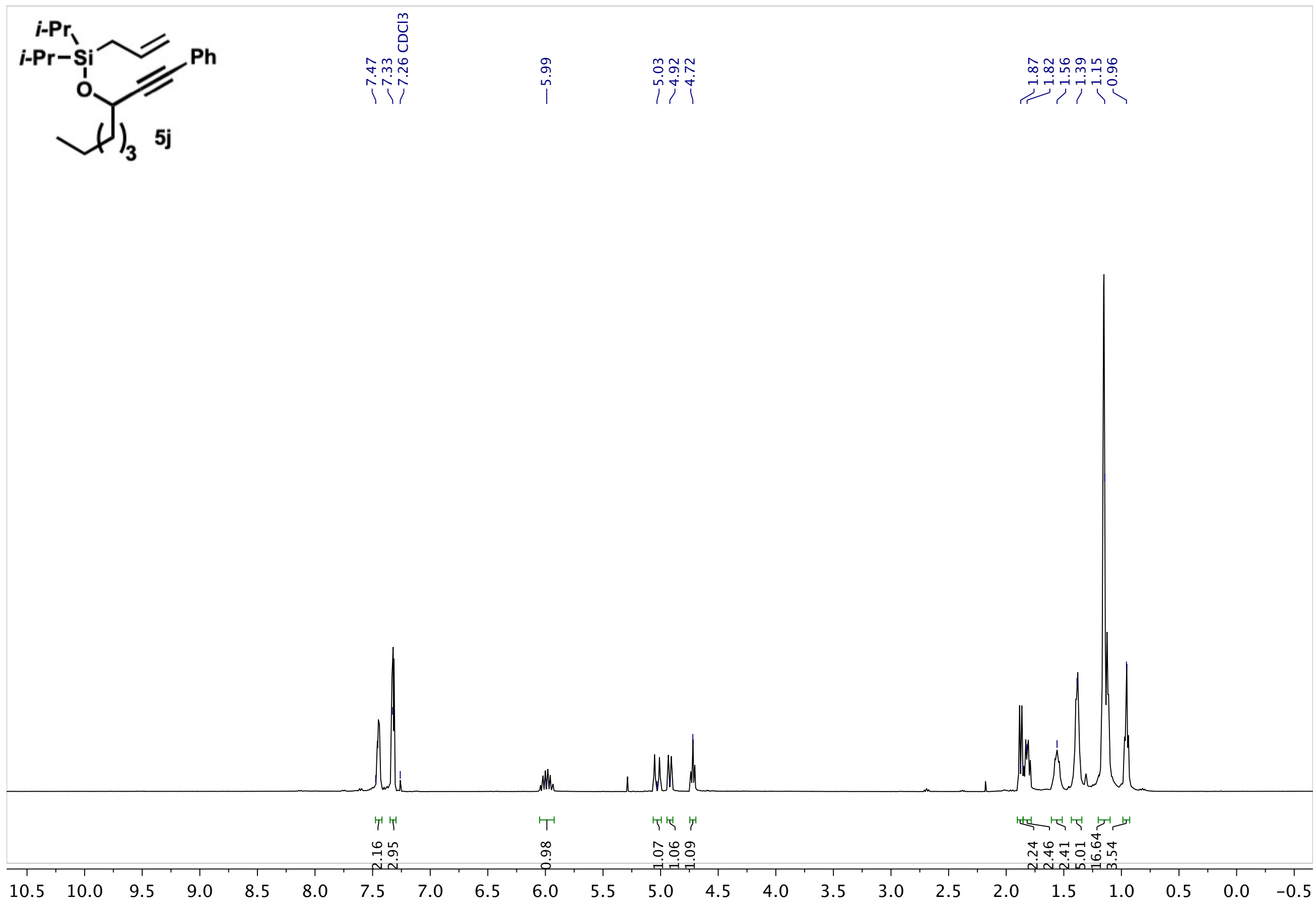

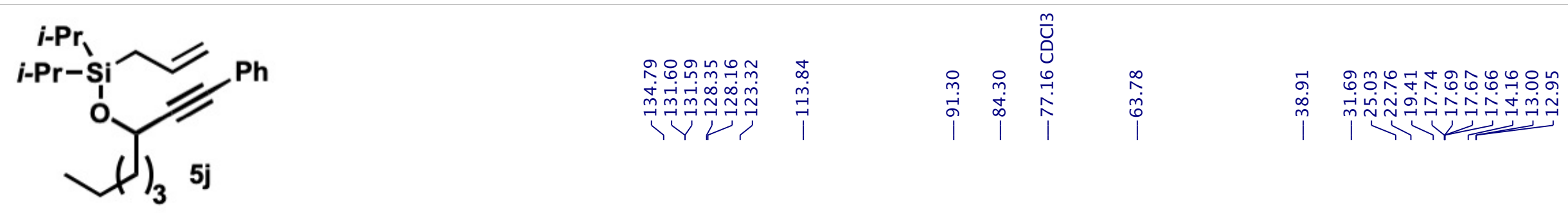

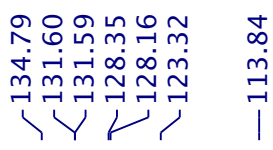

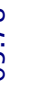

m m

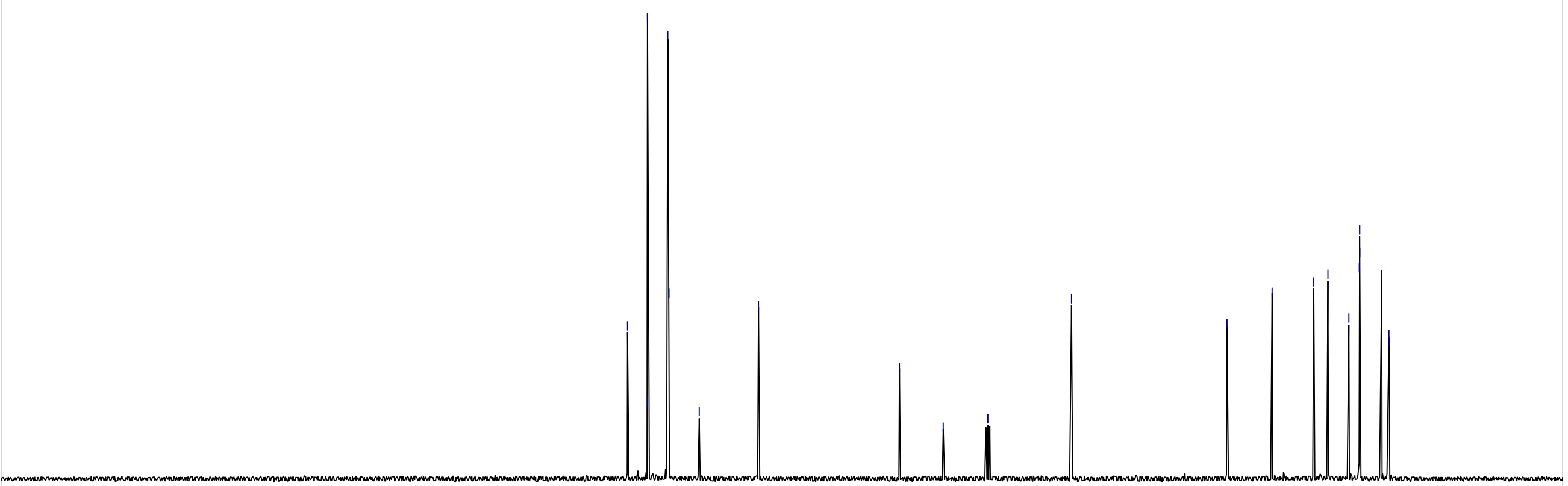



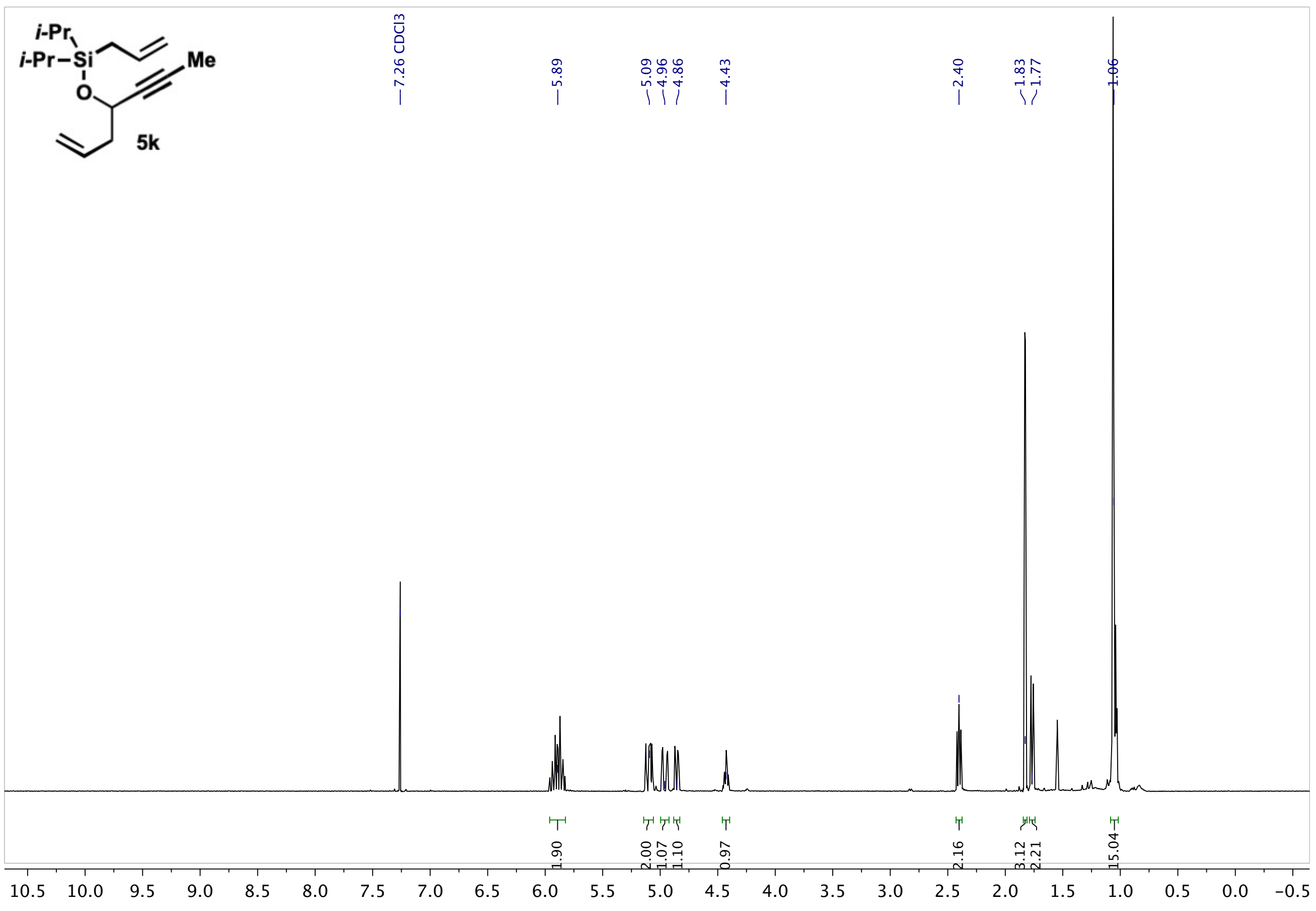

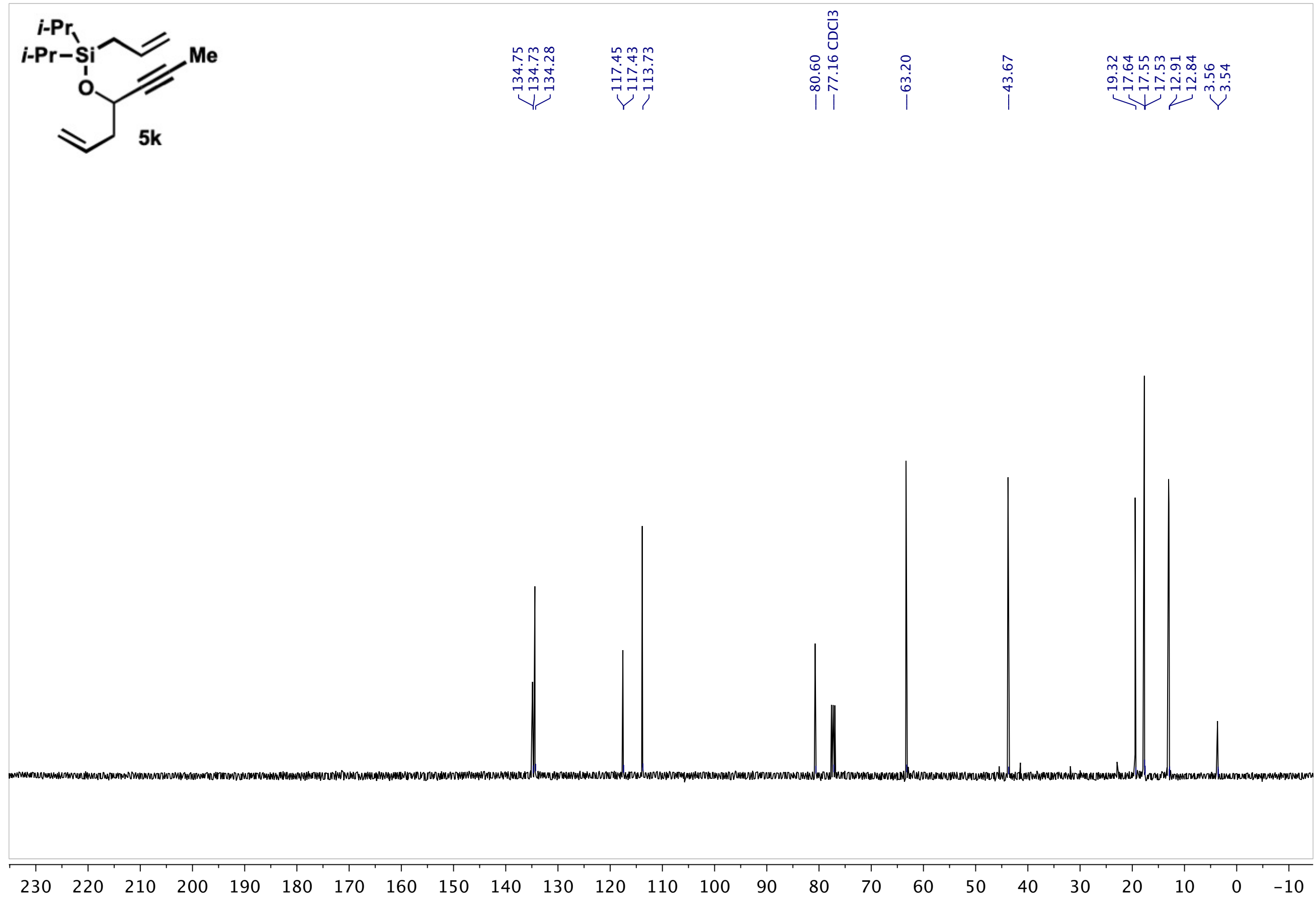

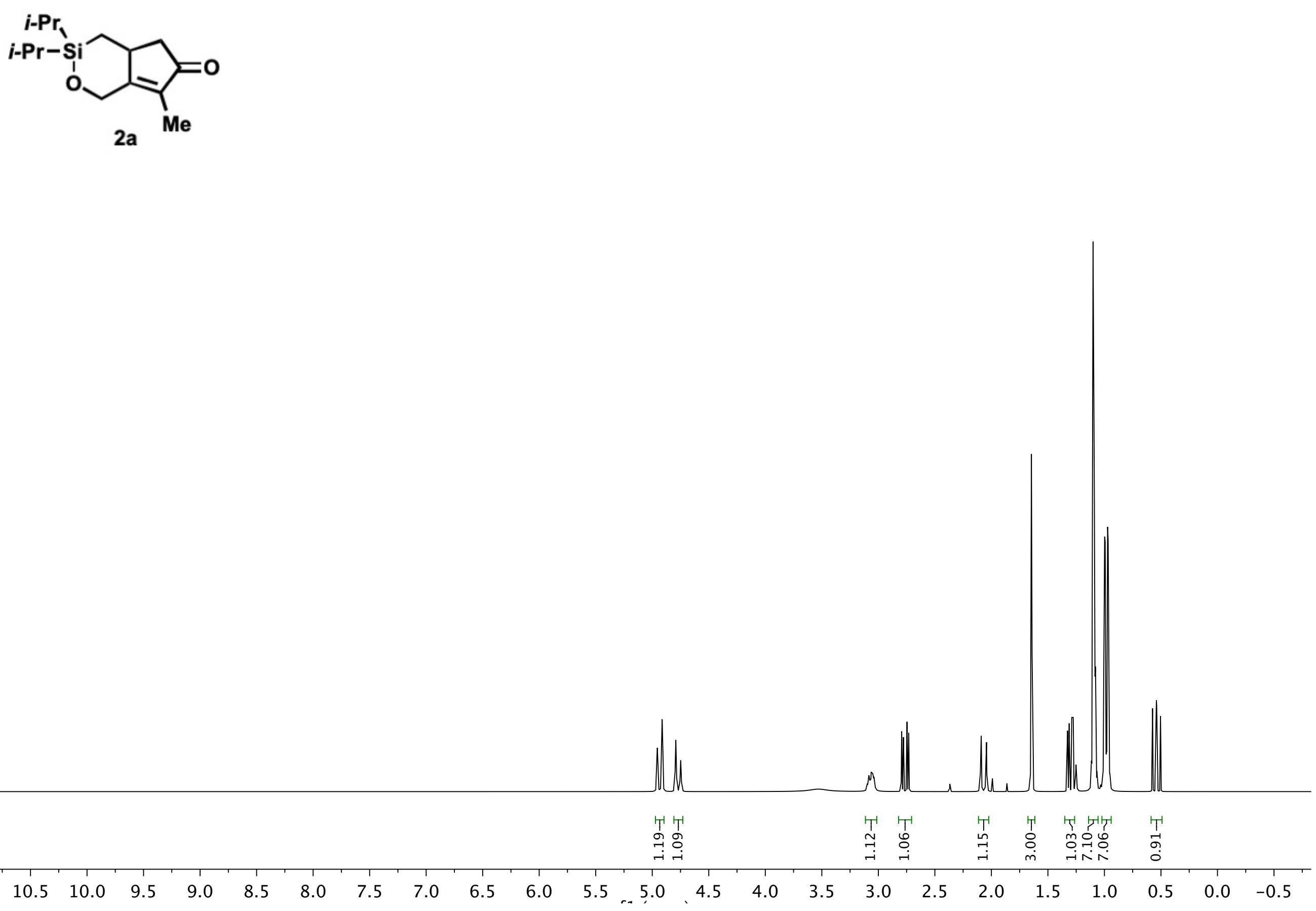


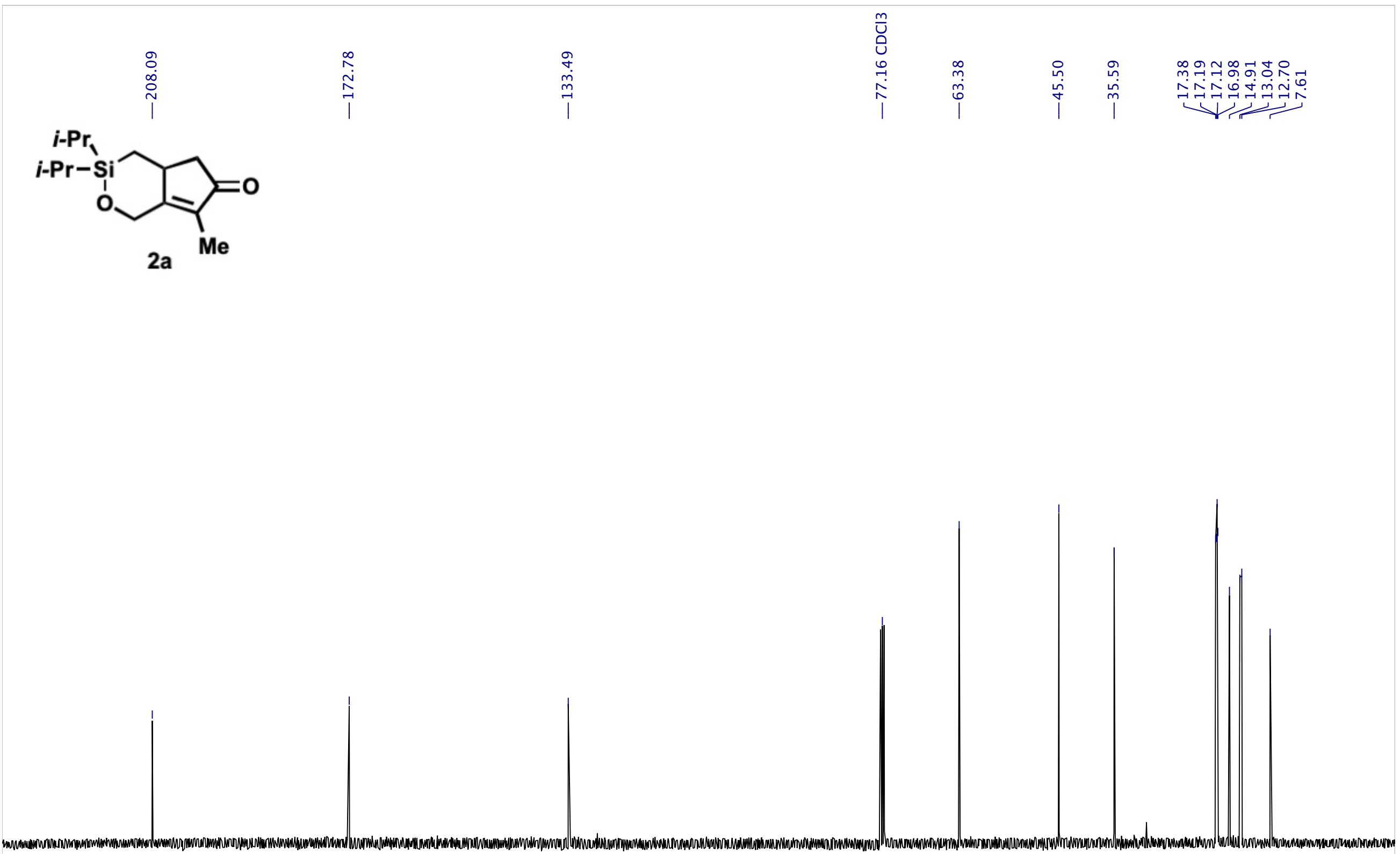

$\begin{array}{lllllllllllllllllllllllll}230 & 220 & 210 & 200 & 190 & 180 & 170 & 160 & 150 & 140 & 130 & 120 & 110 & 100 & 90 & 80 & 70 & 60 & 50 & 40 & 30 & 20 & 10 & 0 & -10\end{array}$




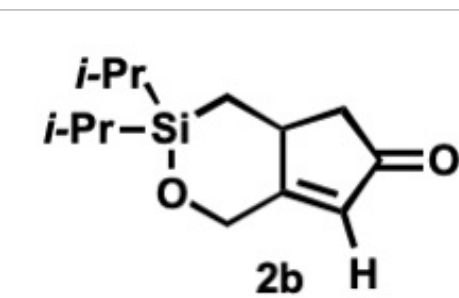

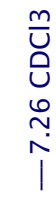

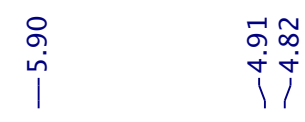

$2 b$

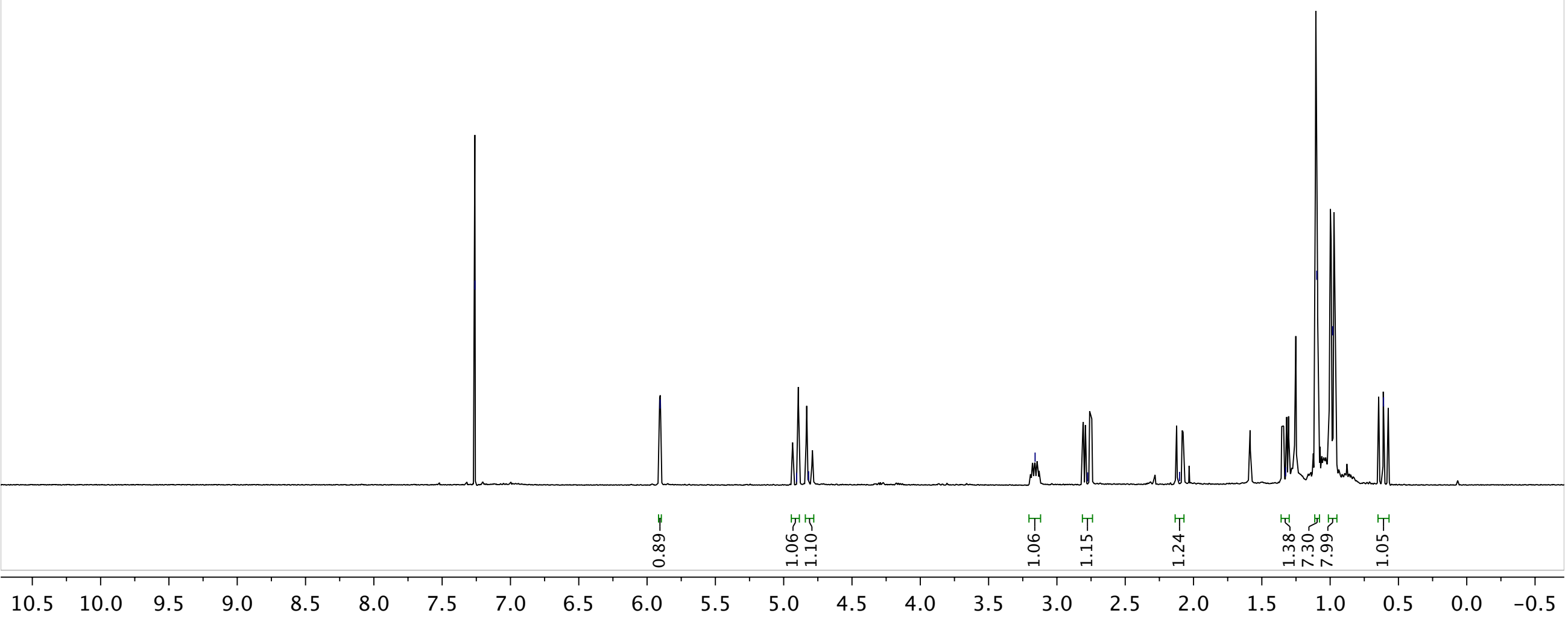



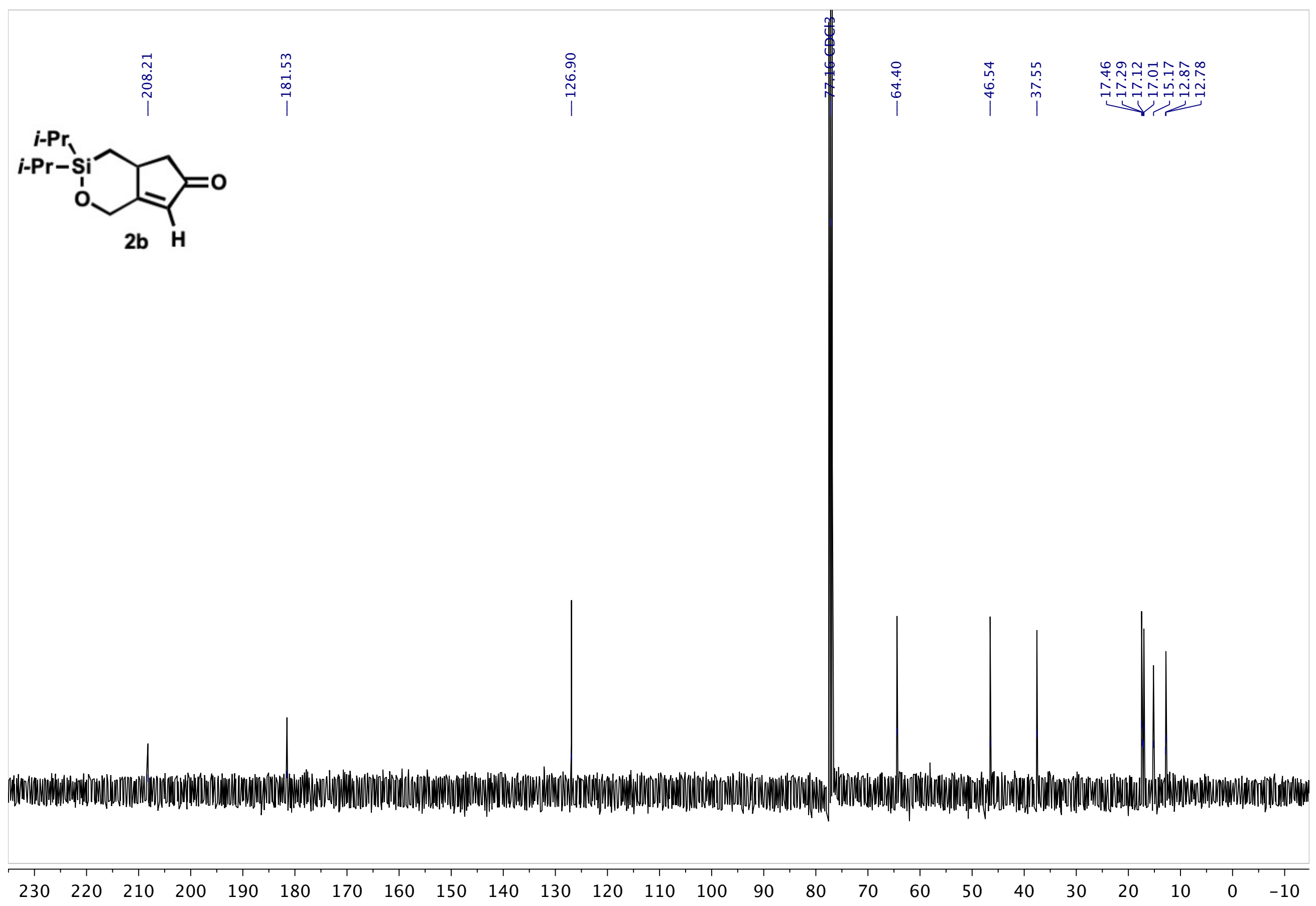

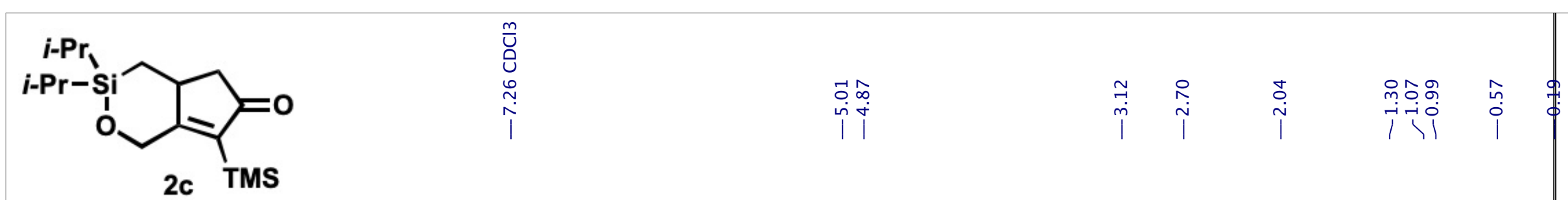

2c

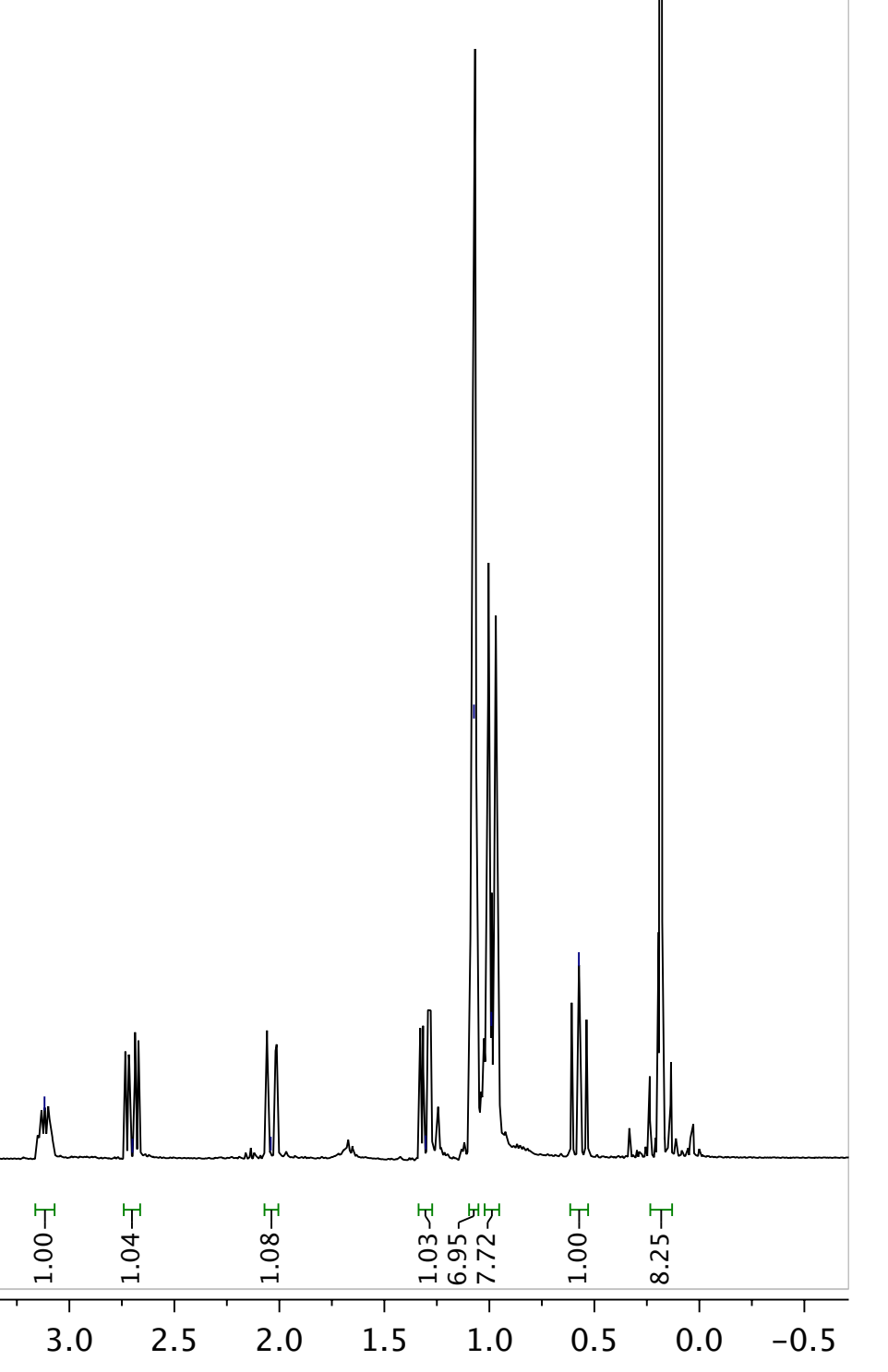



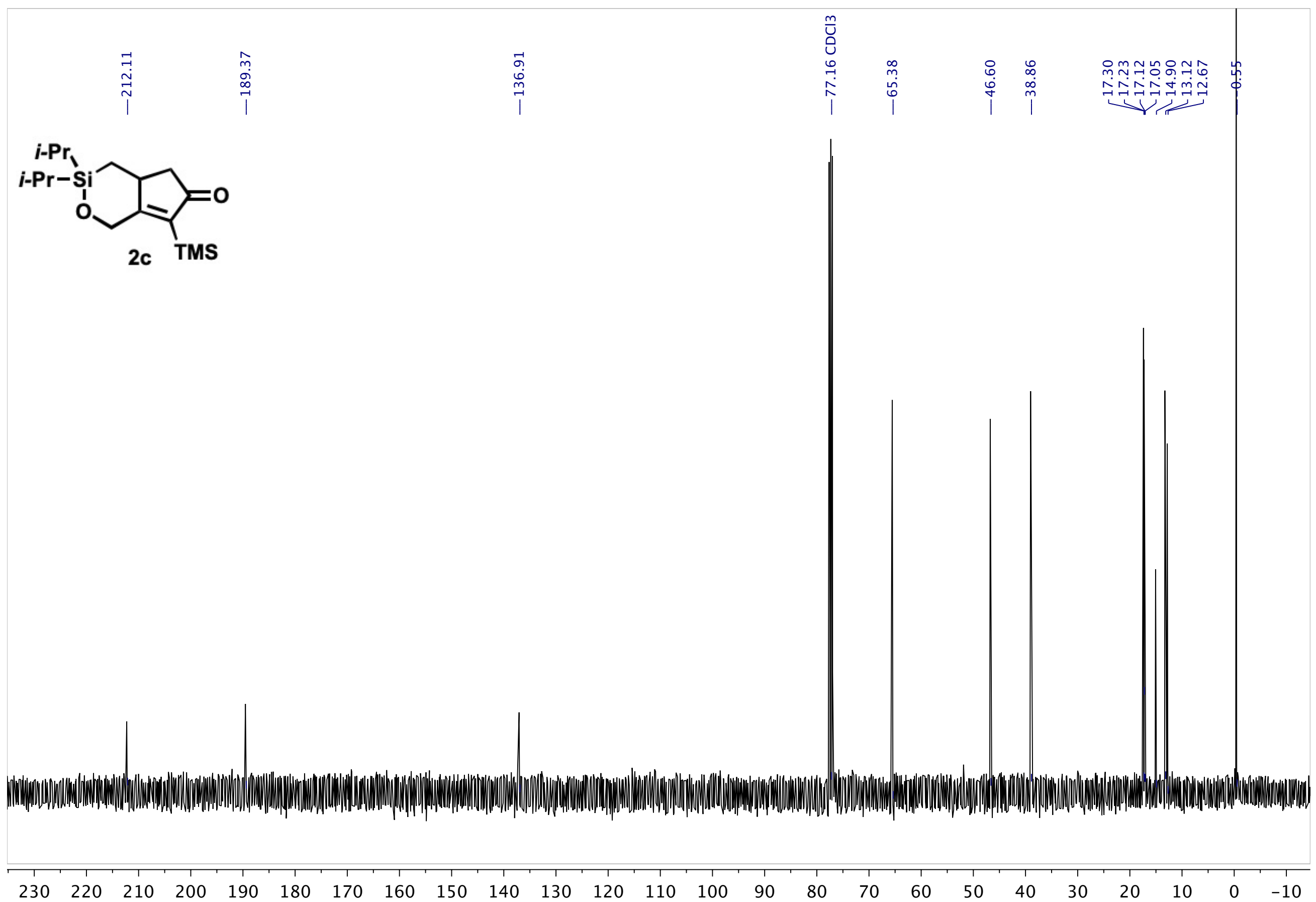

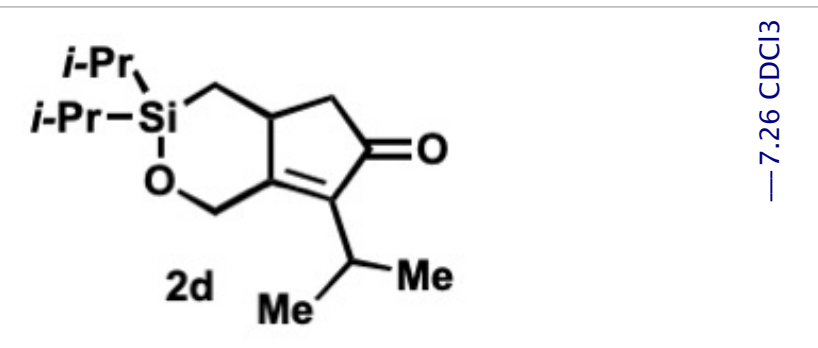

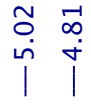

$\begin{array}{ll}-1 & 0 \\ 0 & \stackrel{0}{1}\end{array}$

$\stackrel{m}{o}$

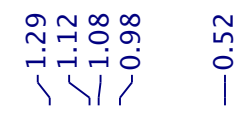

2d

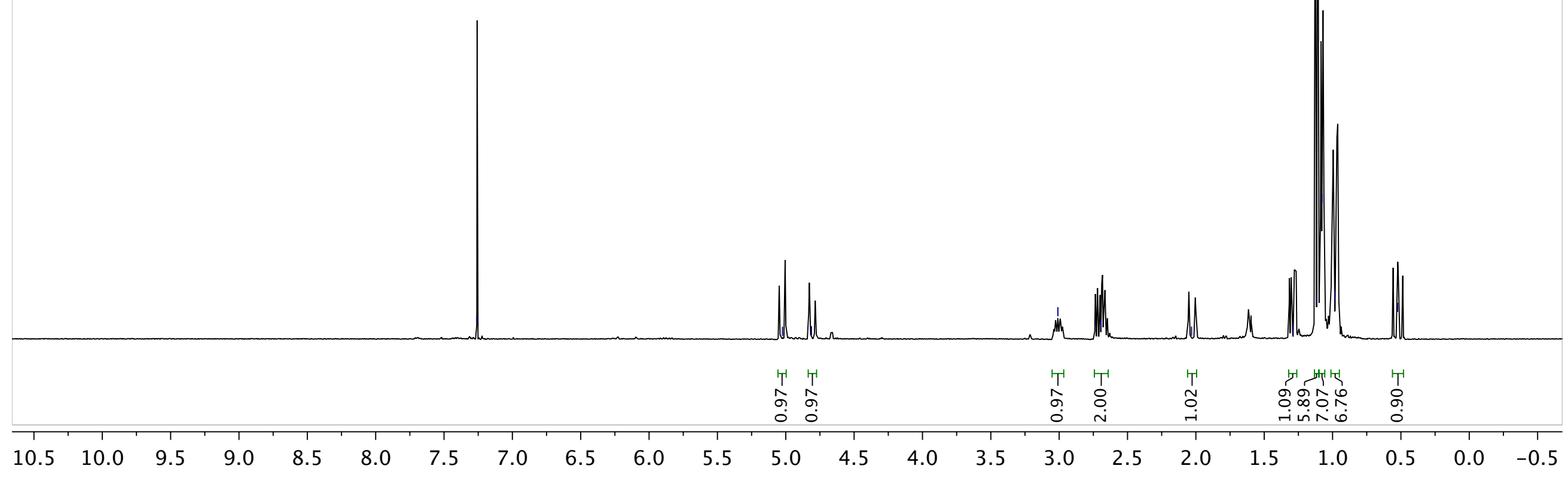




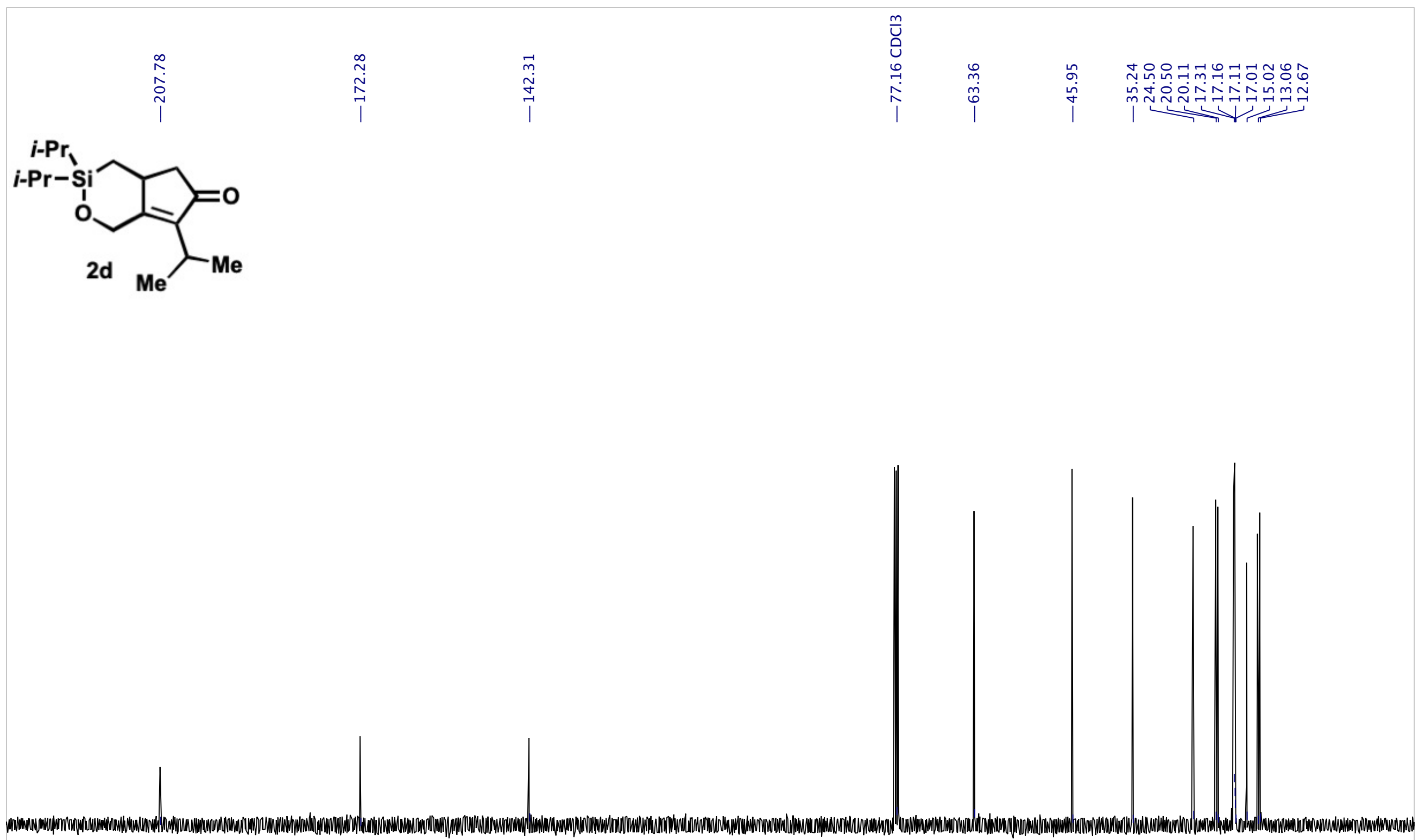

$\begin{array}{lllllllllllllllllllllllllllll}230 & 220 & 210 & 200 & 190 & 180 & 170 & 160 & 150 & 140 & 130 & 120 & 110 & 100 & 90 & 80 & 70 & 60 & 50 & 40 & 30 & 20 & 10 & 0 & -10\end{array}$




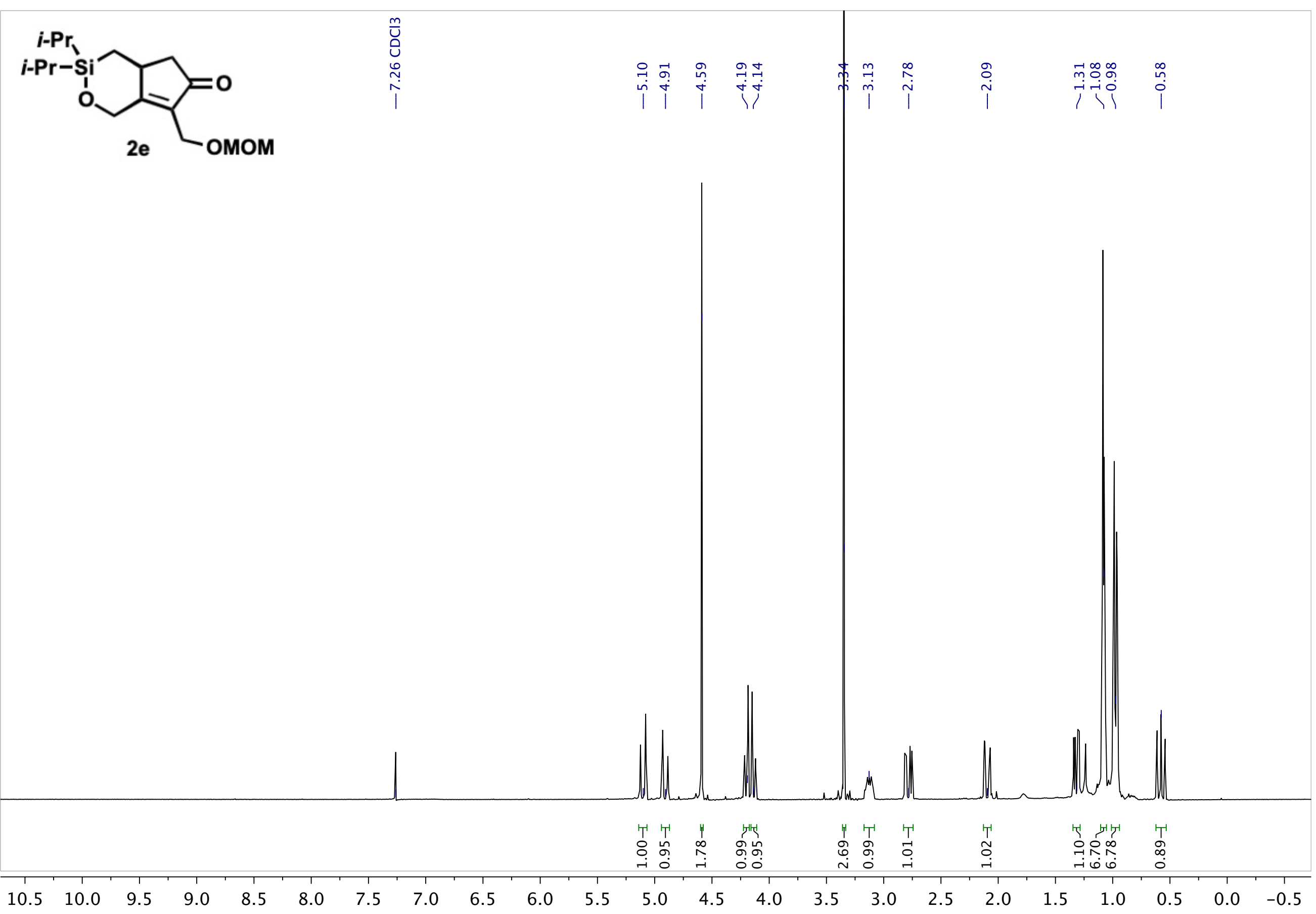




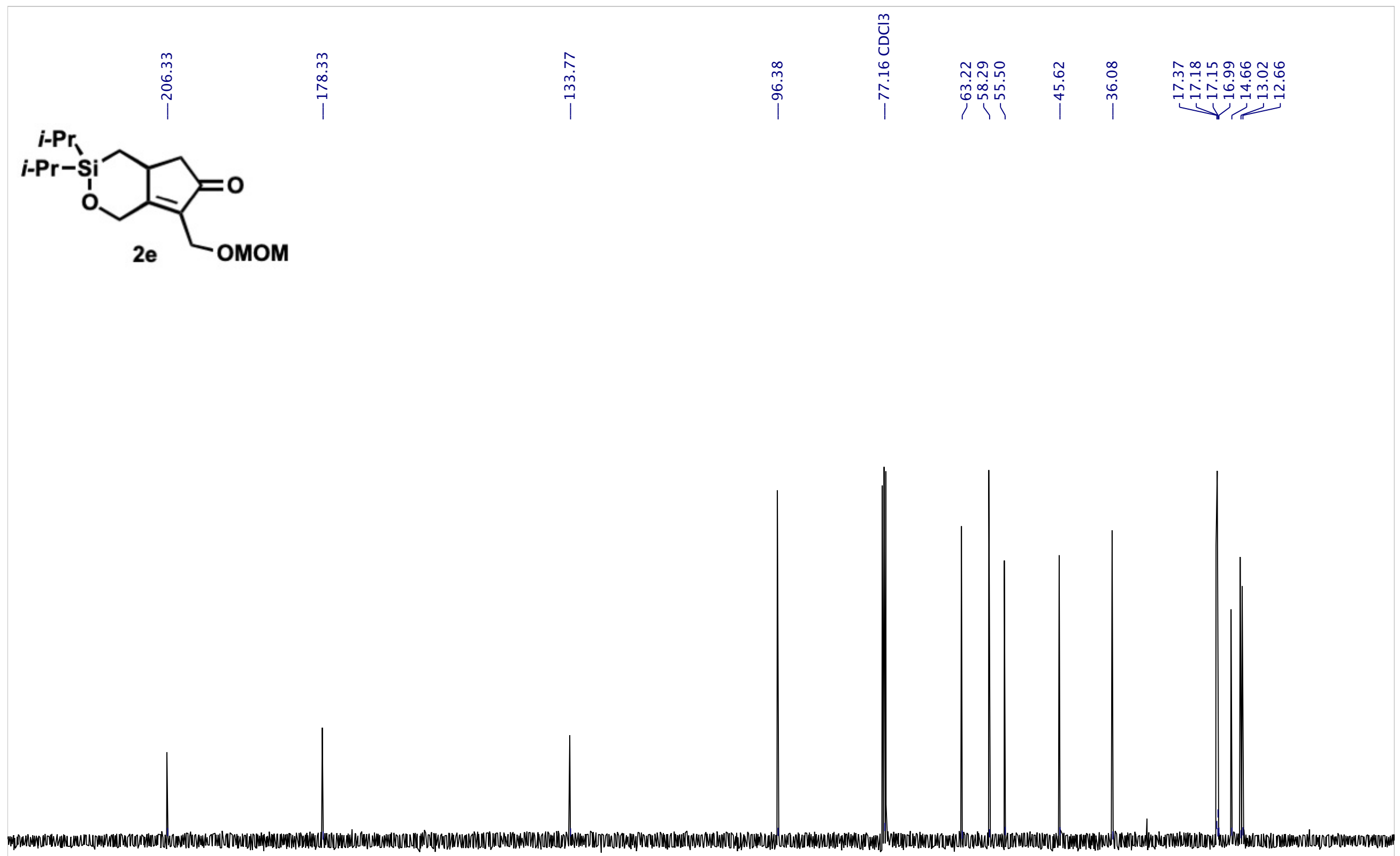

$\begin{array}{lllllllllllllllllllllllllll}230 & 220 & 210 & 200 & 190 & 180 & 170 & 160 & 150 & 140 & 130 & 120 & 110 & 100 & 90 & 80 & 70 & 60 & 50 & 40 & 30 & 20 & 10 & 0 & -10\end{array}$



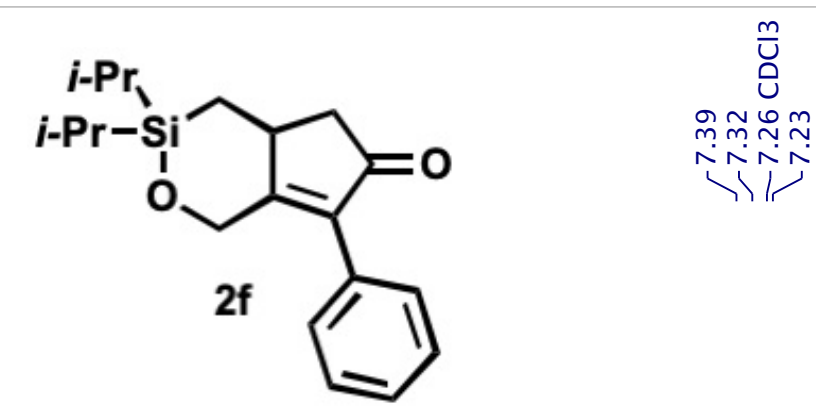

ㄷำ

กิं

$\stackrel{m}{\stackrel{m}{m}} \stackrel{m}{i}$

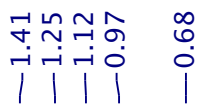

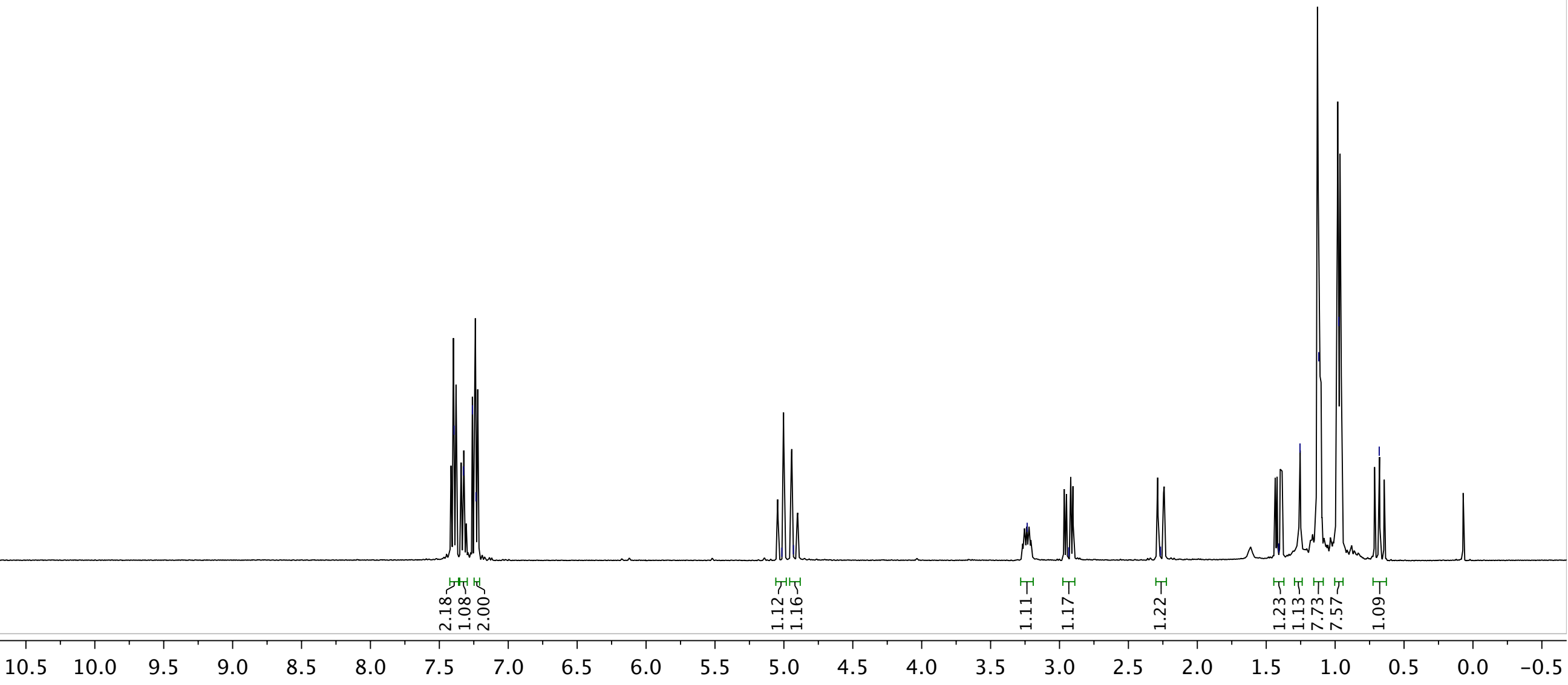




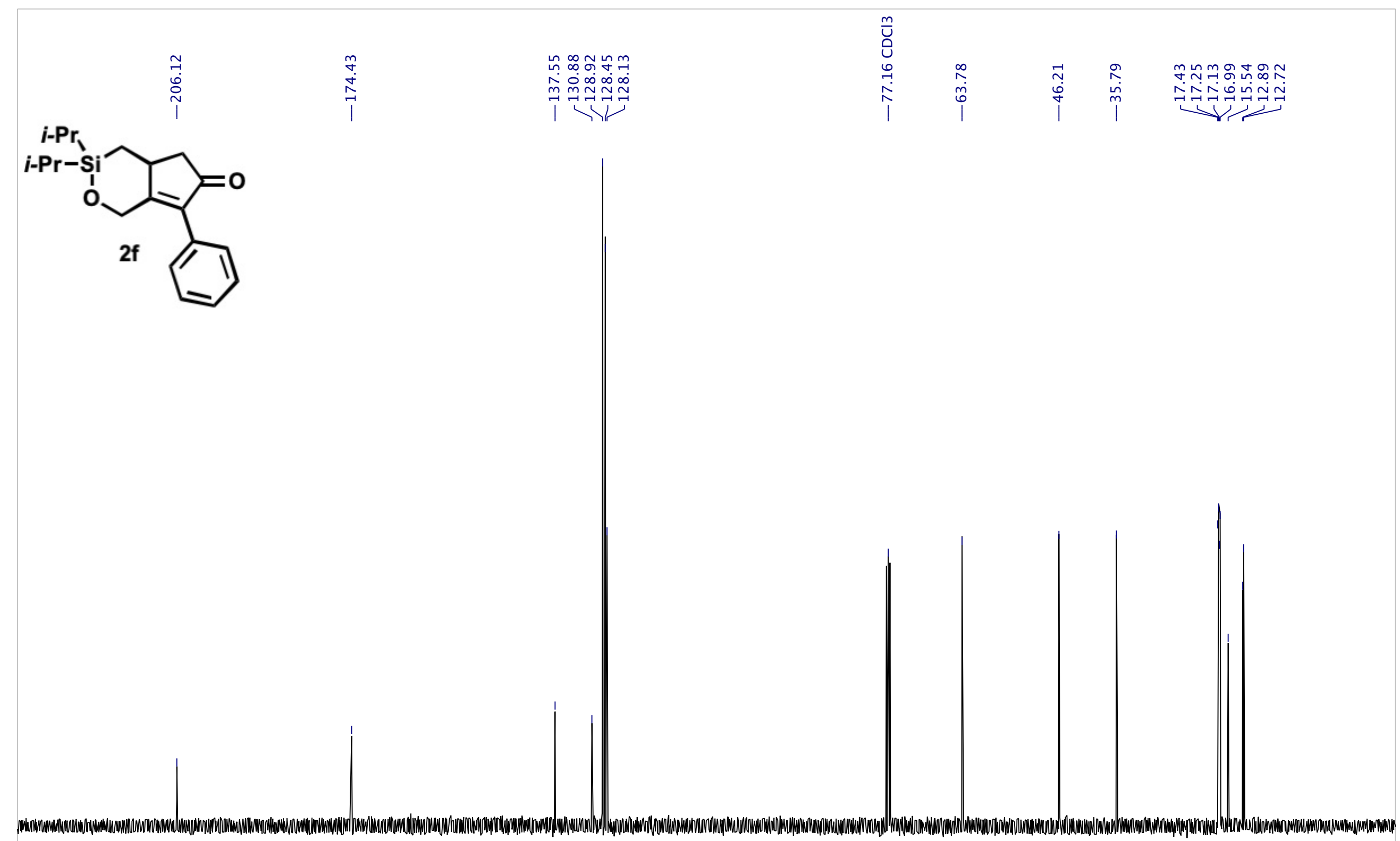

$\begin{array}{llllllllllllllllllllllllllll}230 & 220 & 210 & 200 & 190 & 180 & 170 & 160 & 150 & 140 & 130 & 120 & 110 & 100 & 90 & 80 & 70 & 60 & 50 & 40 & 30 & 20 & 10 & 0 & -10\end{array}$




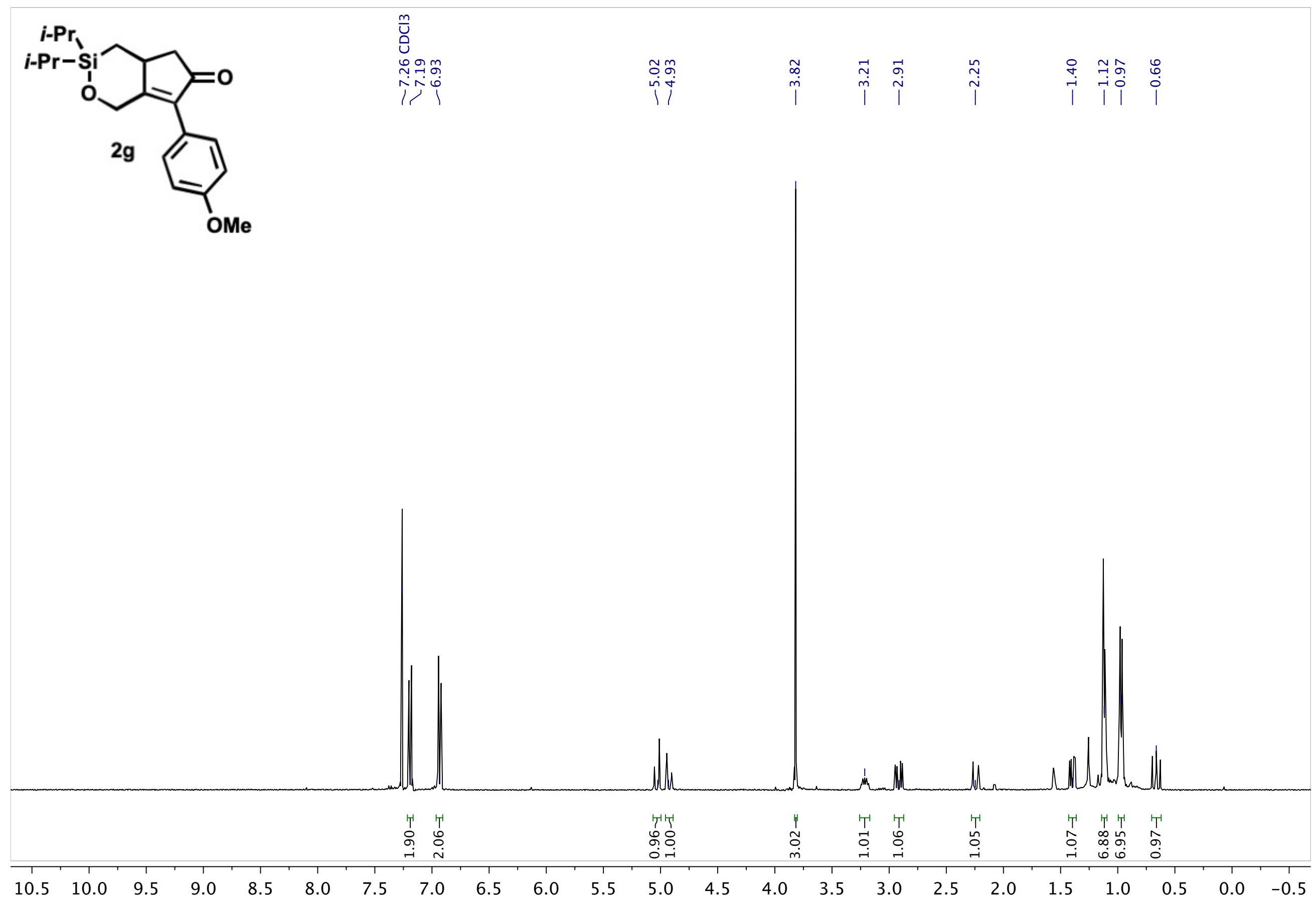




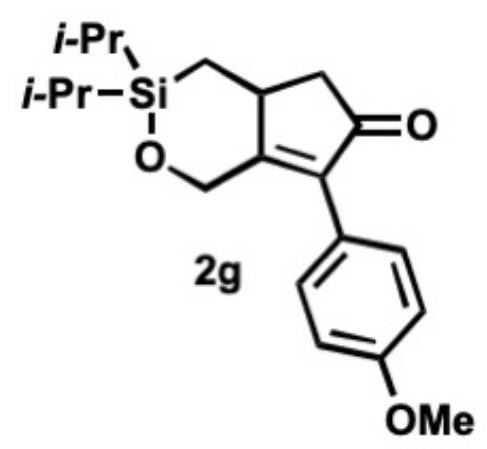



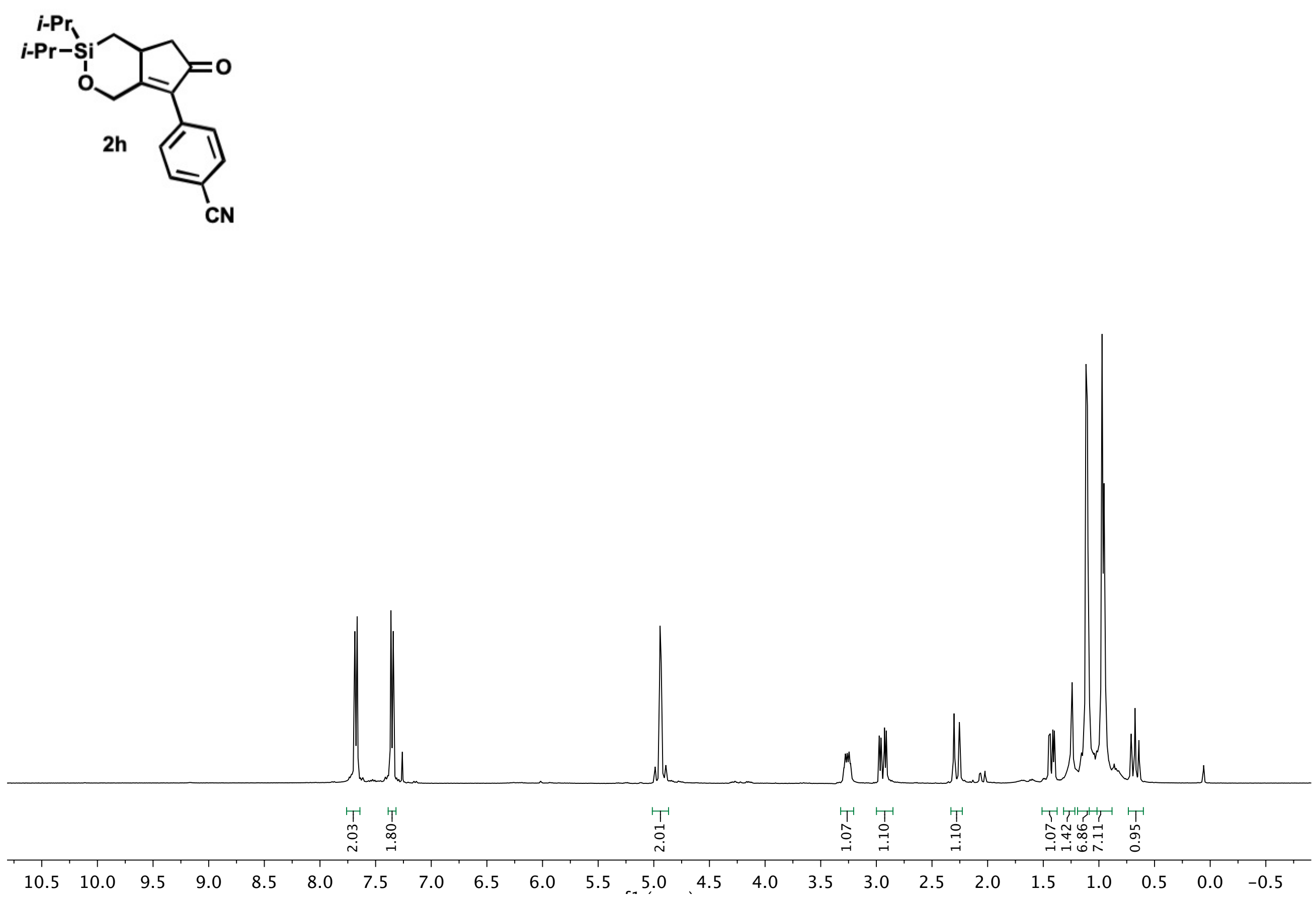


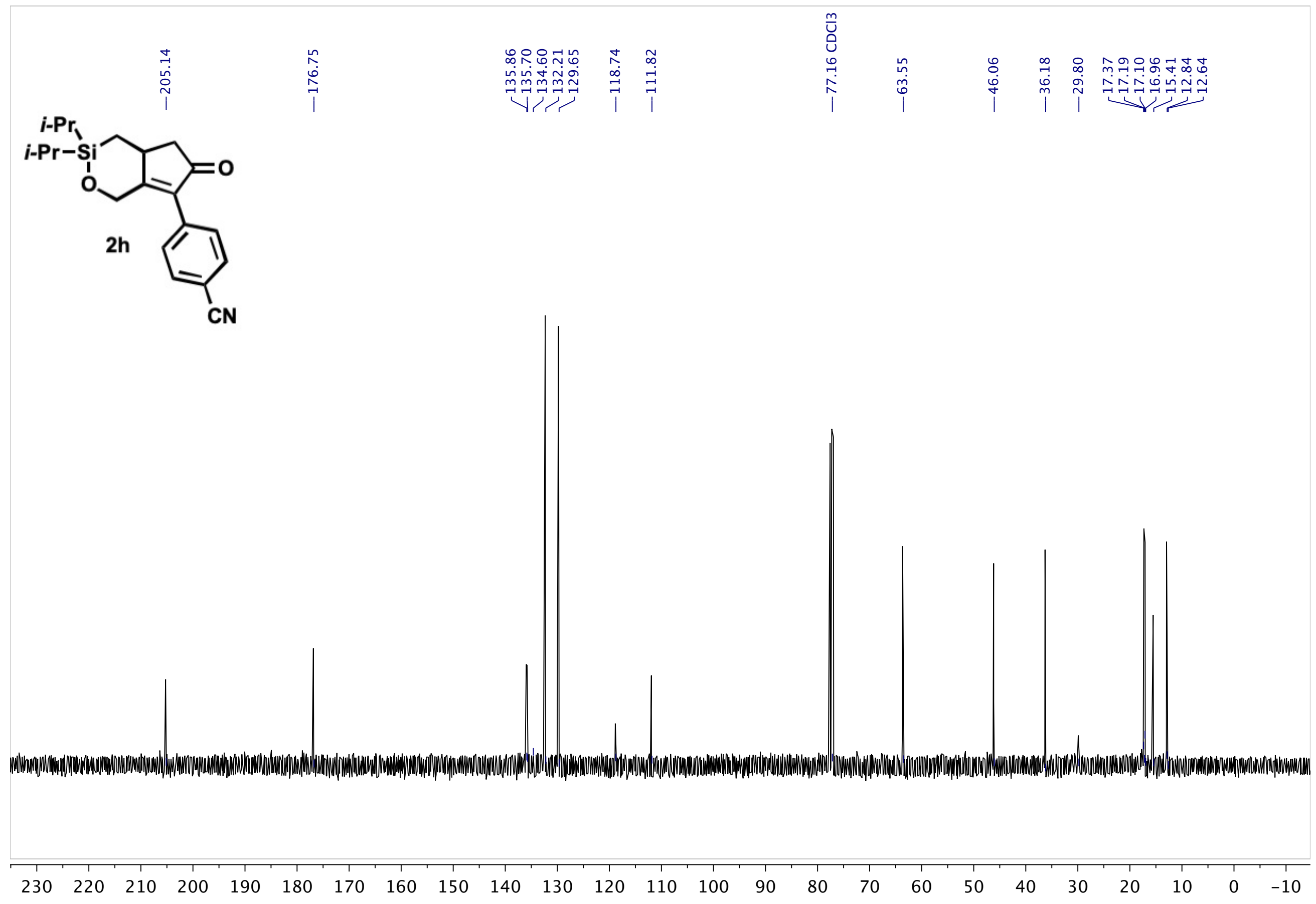



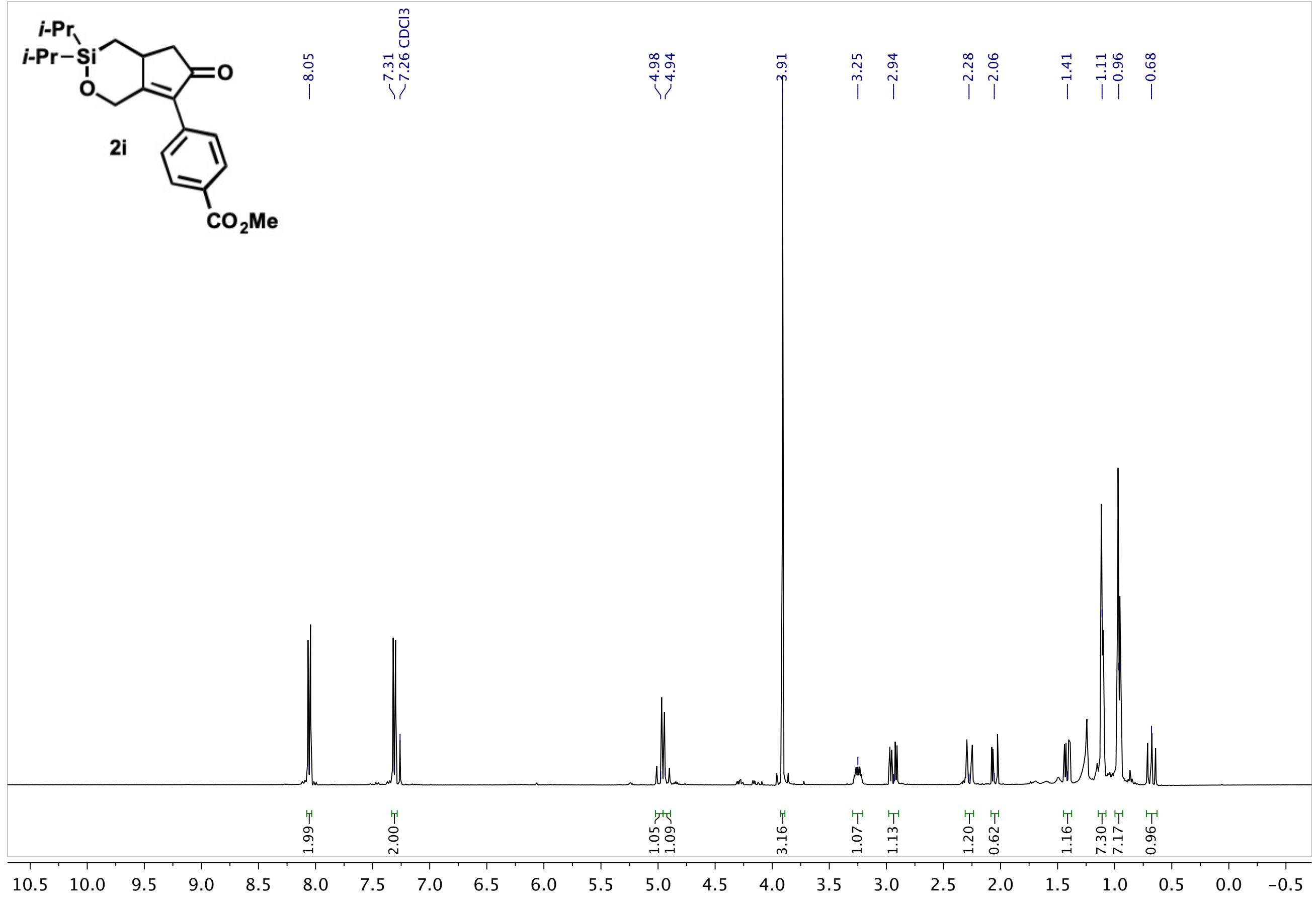


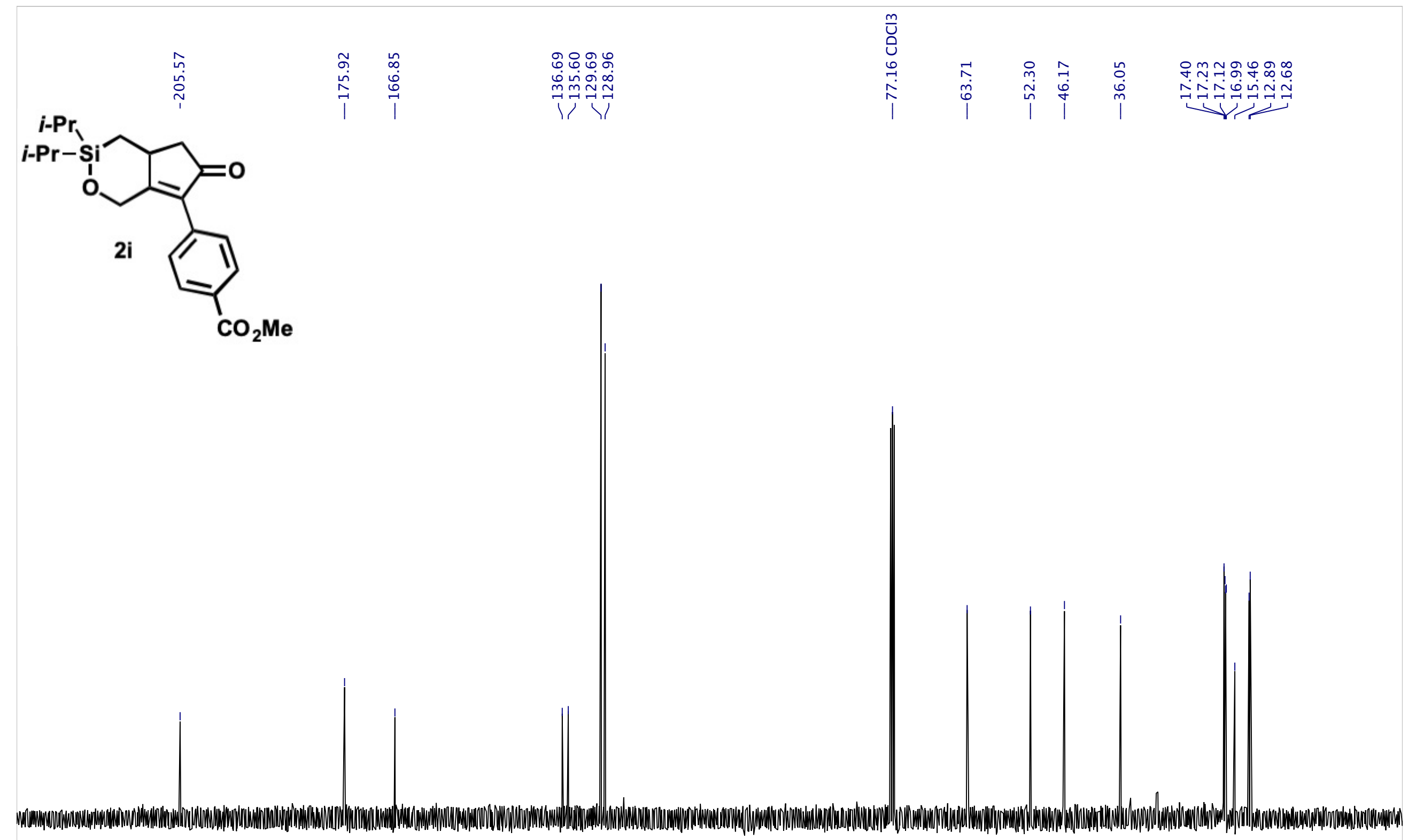

$\begin{array}{llllllllllllllllllllllllllll}230 & 220 & 210 & 200 & 190 & 180 & 170 & 160 & 150 & 140 & 130 & 120 & 110 & 100 & 90 & 80 & 70 & 60 & 50 & 40 & 30 & 20 & 10 & 0 & -10\end{array}$




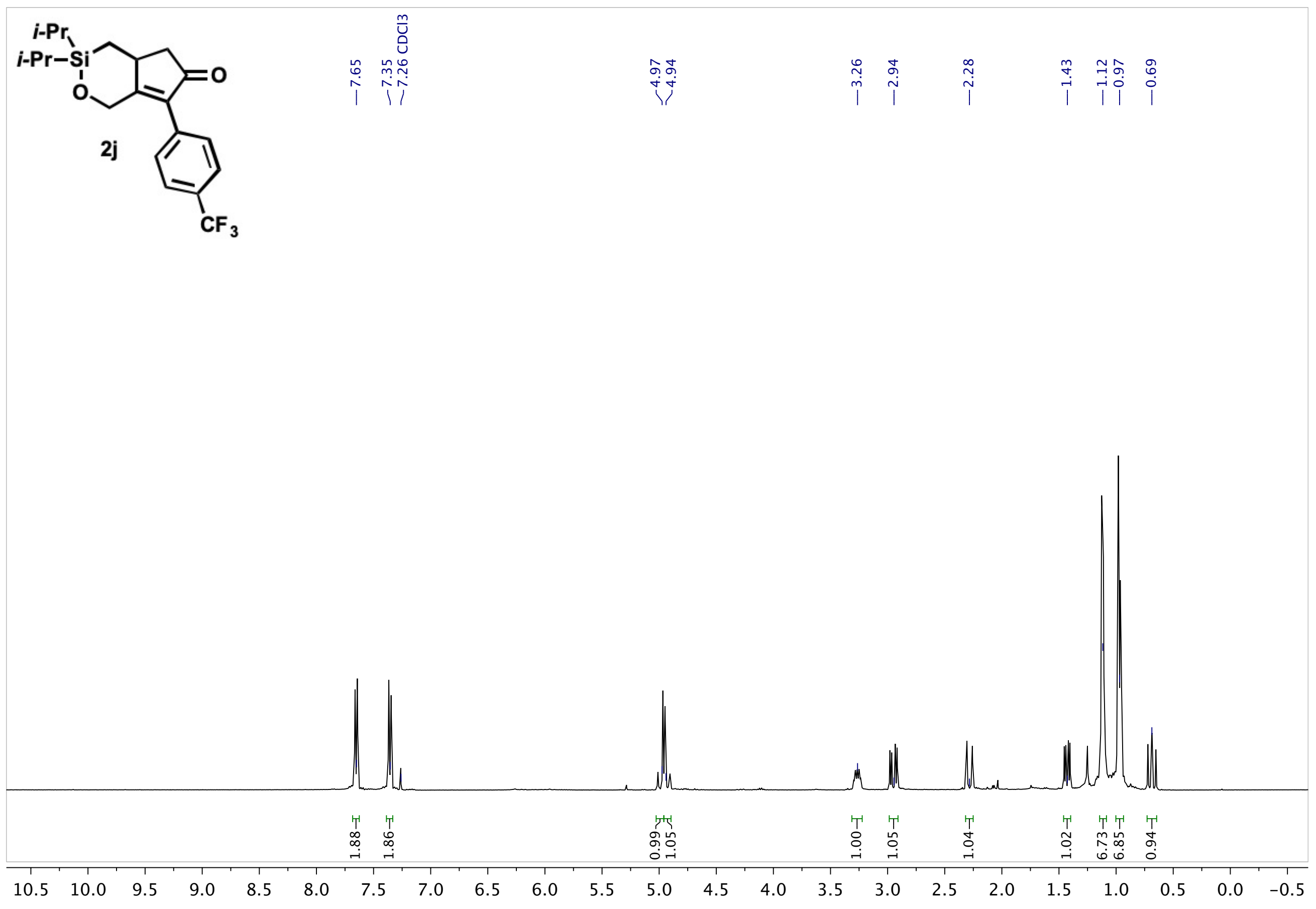



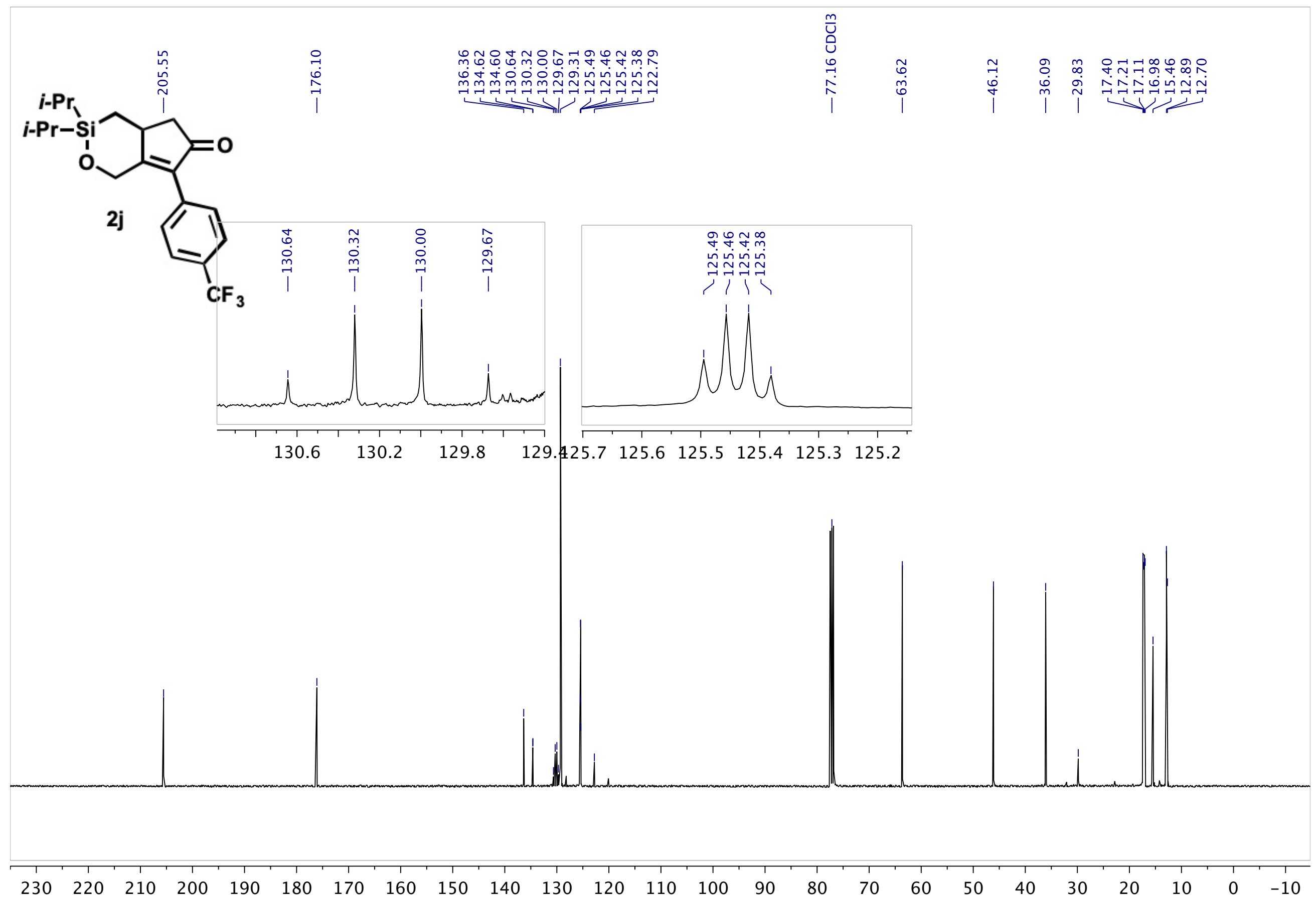


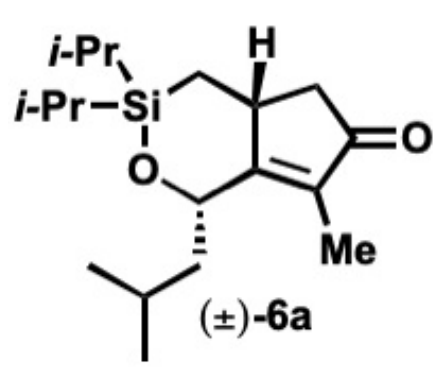

กำ ชุ

$\sqrt[n]{0.0020}$

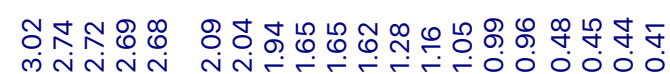

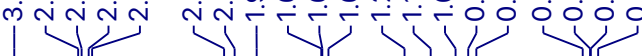

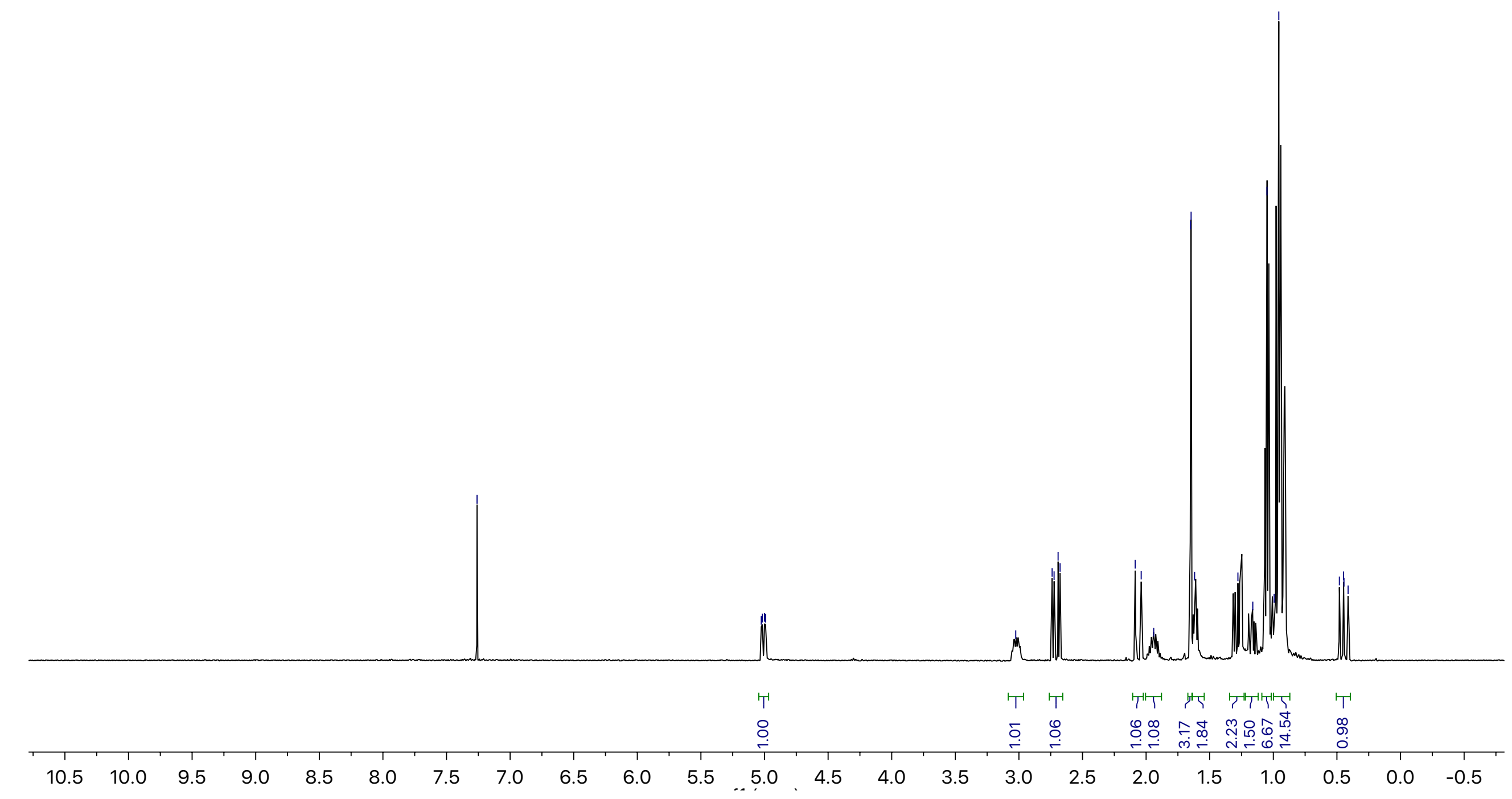




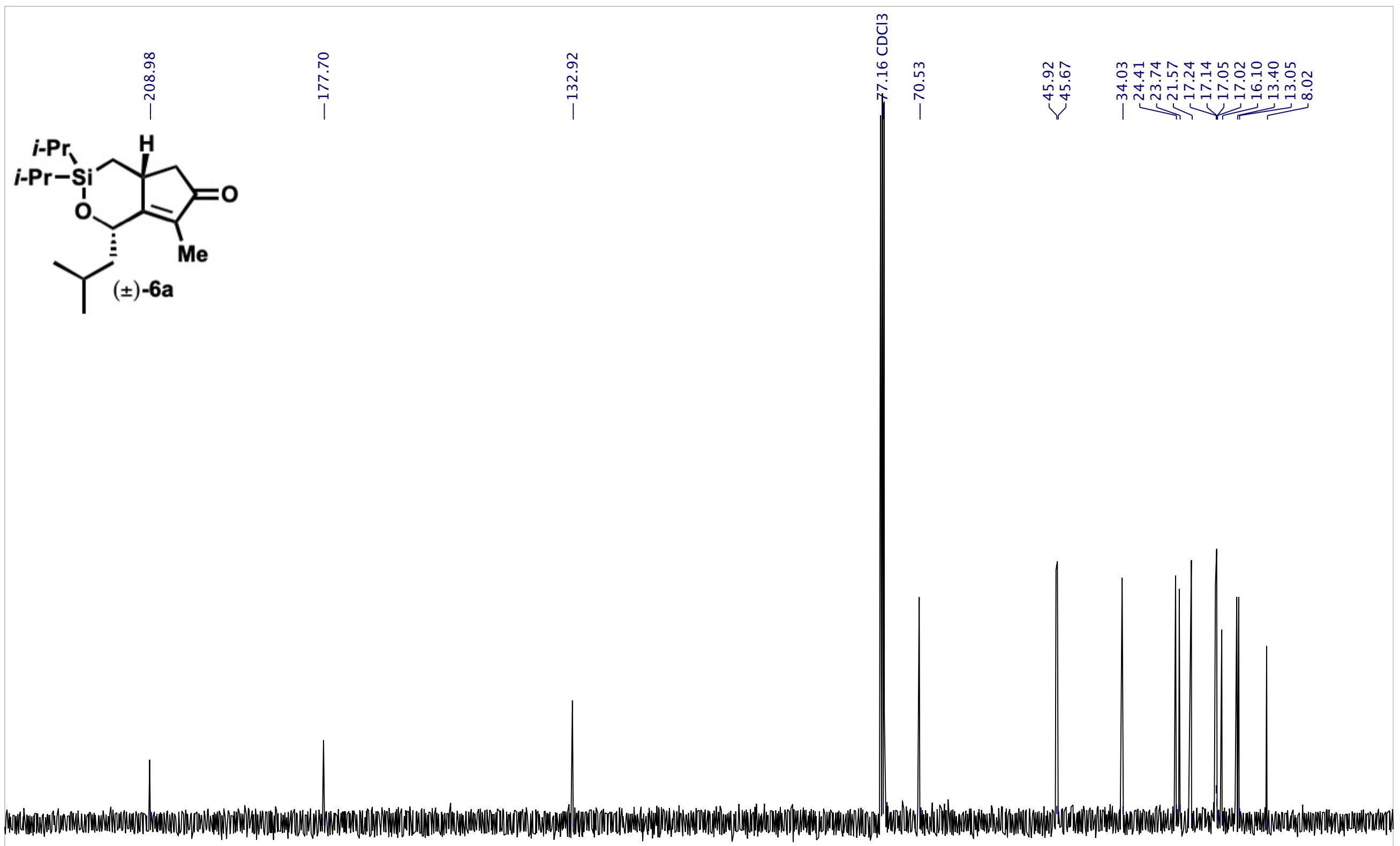

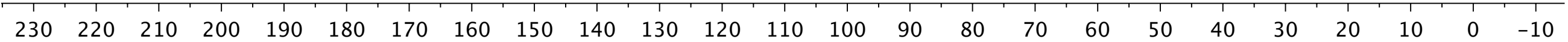




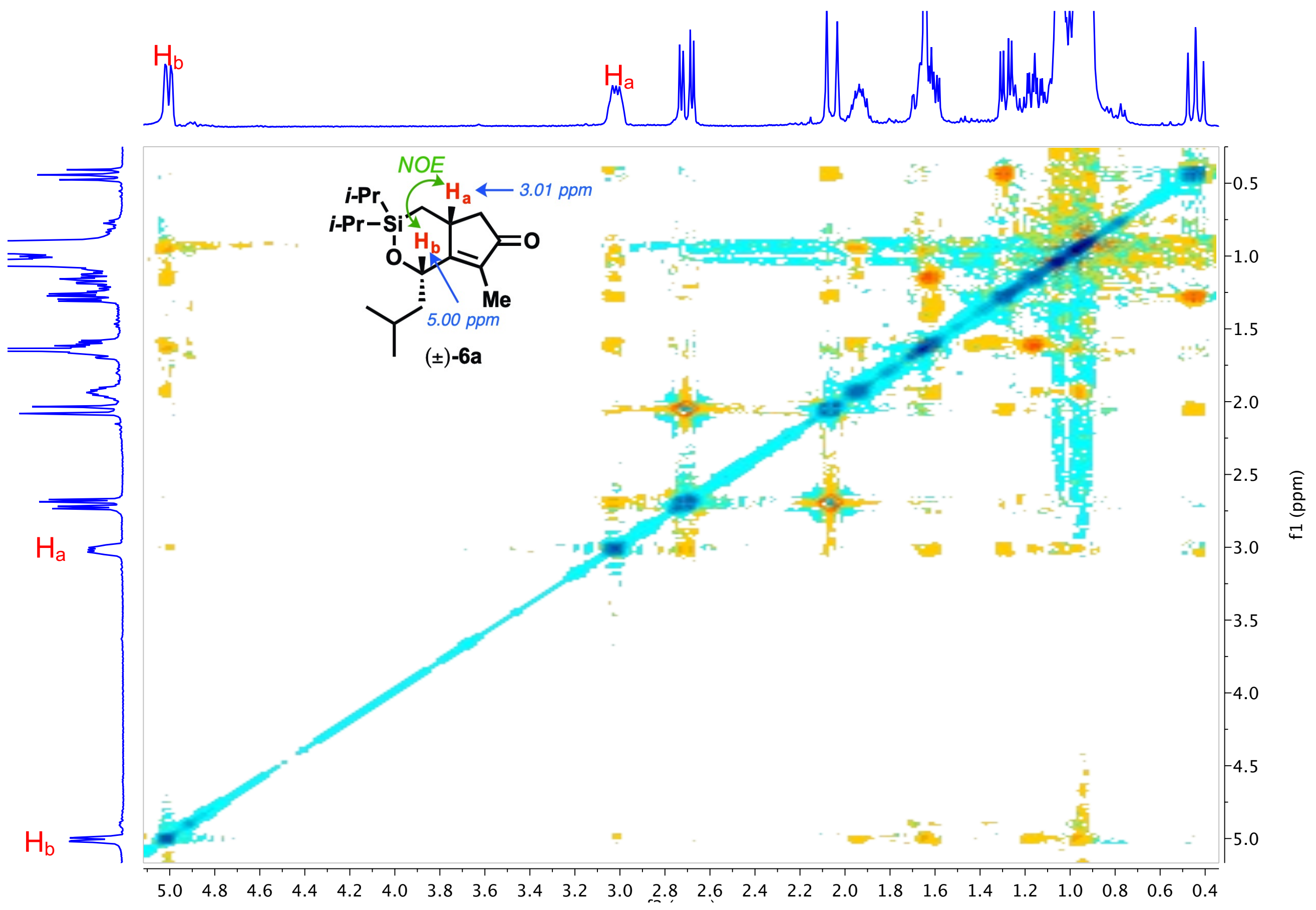




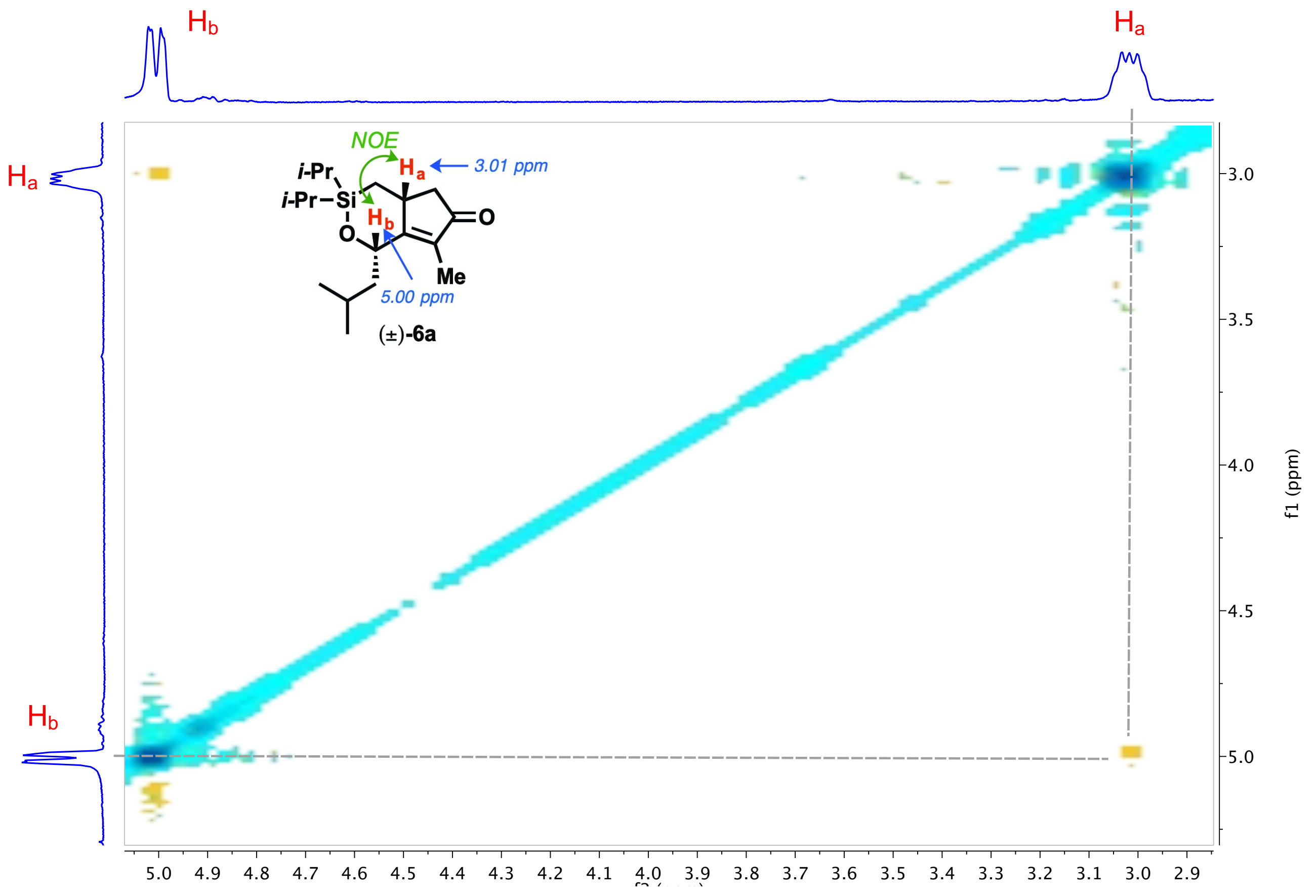




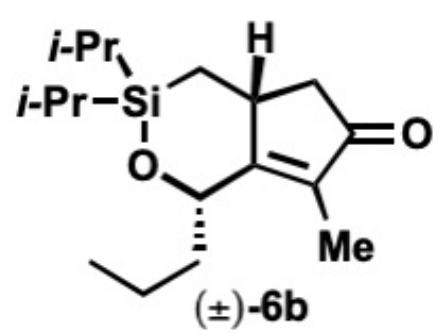

( \pm$)-6 b$

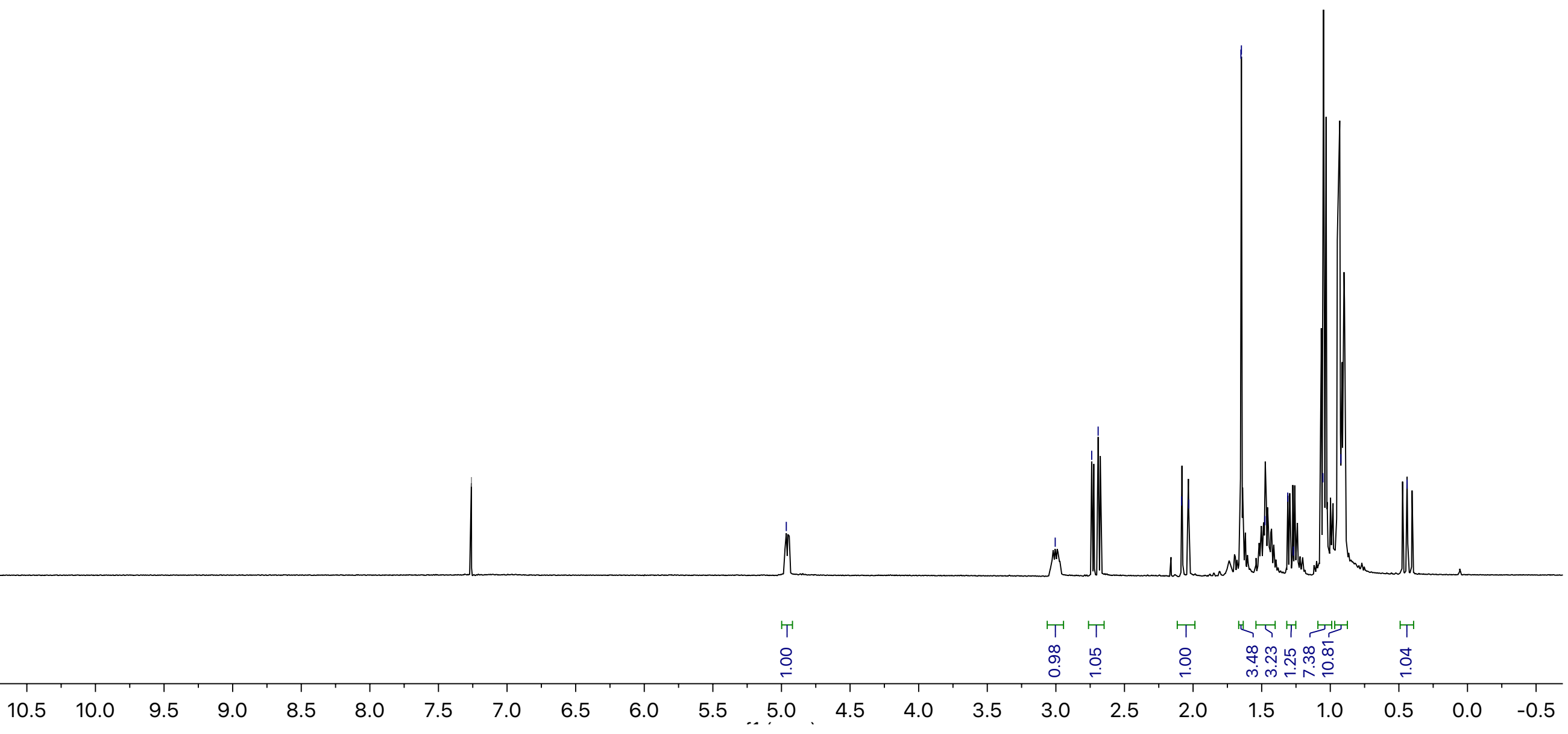




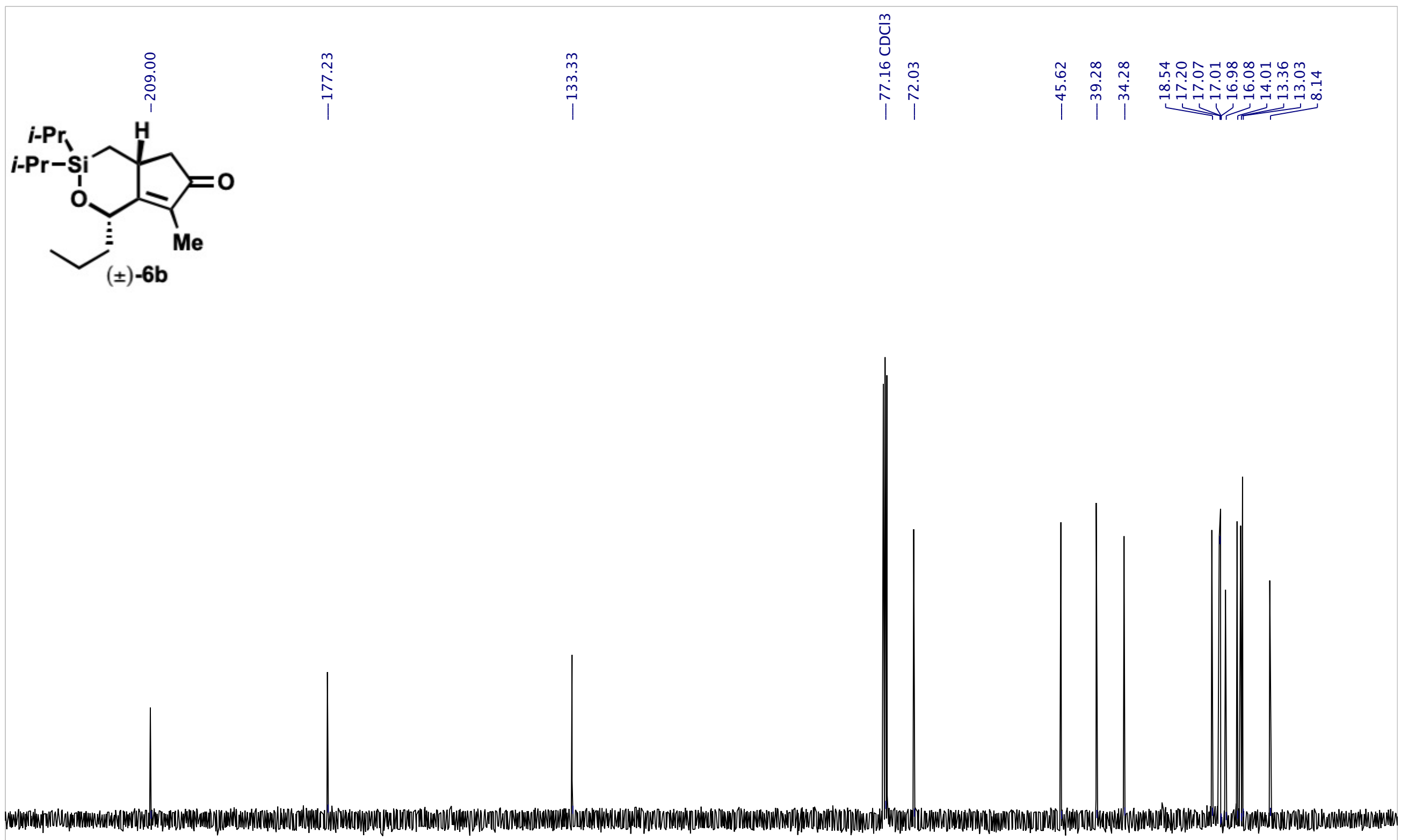

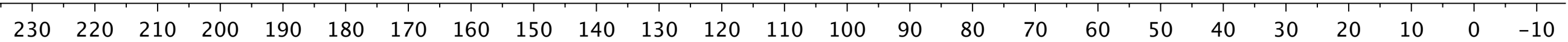




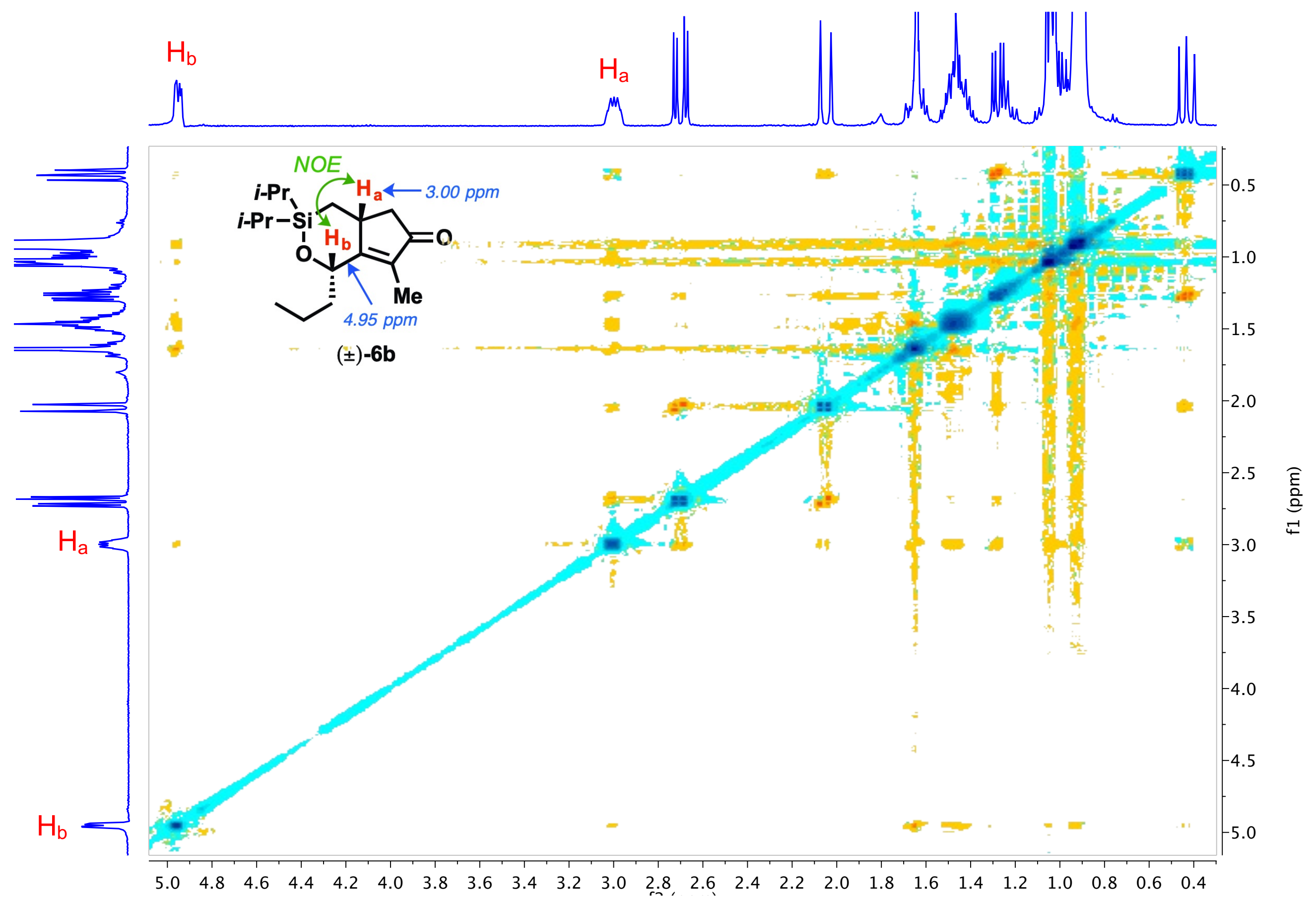




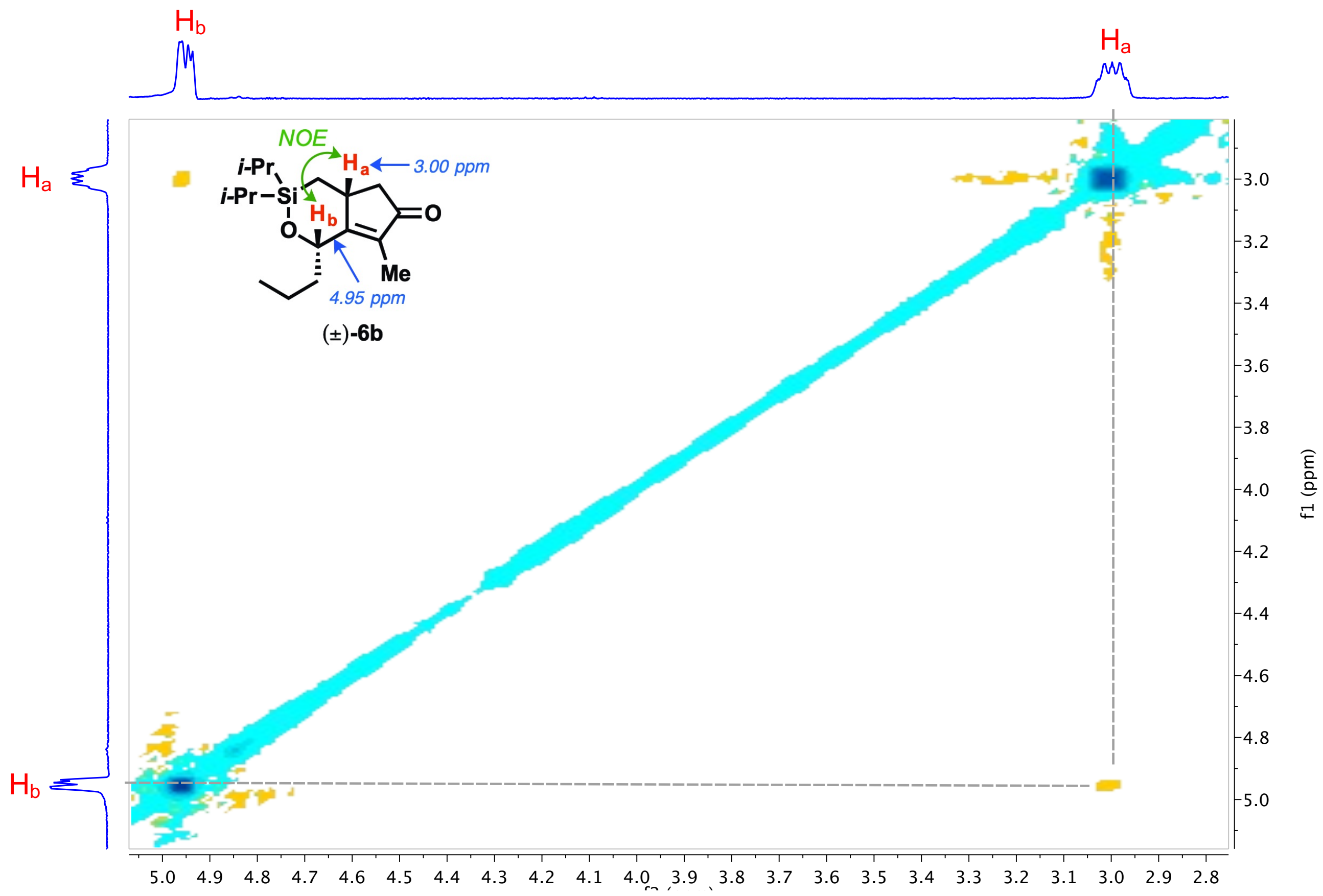



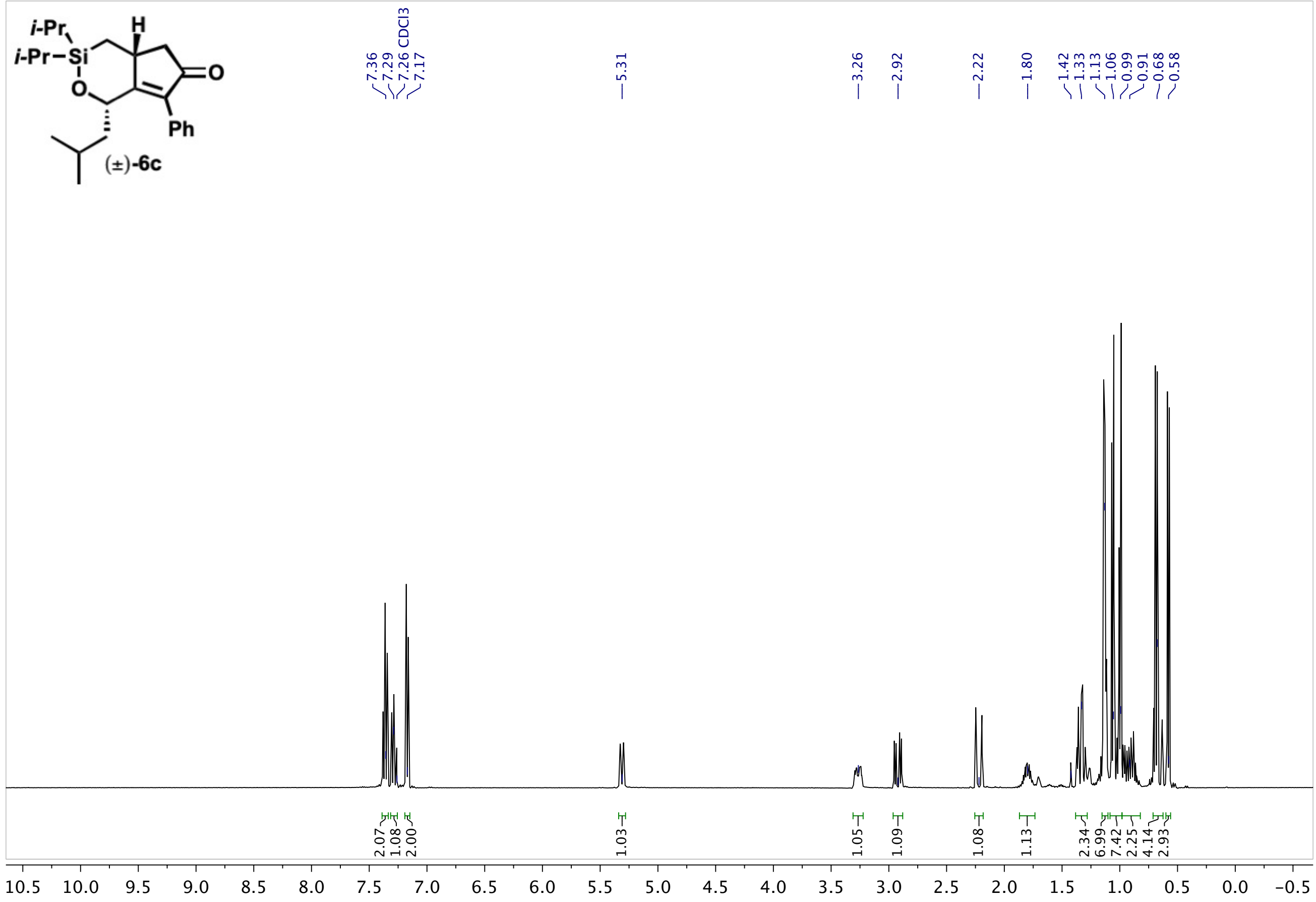


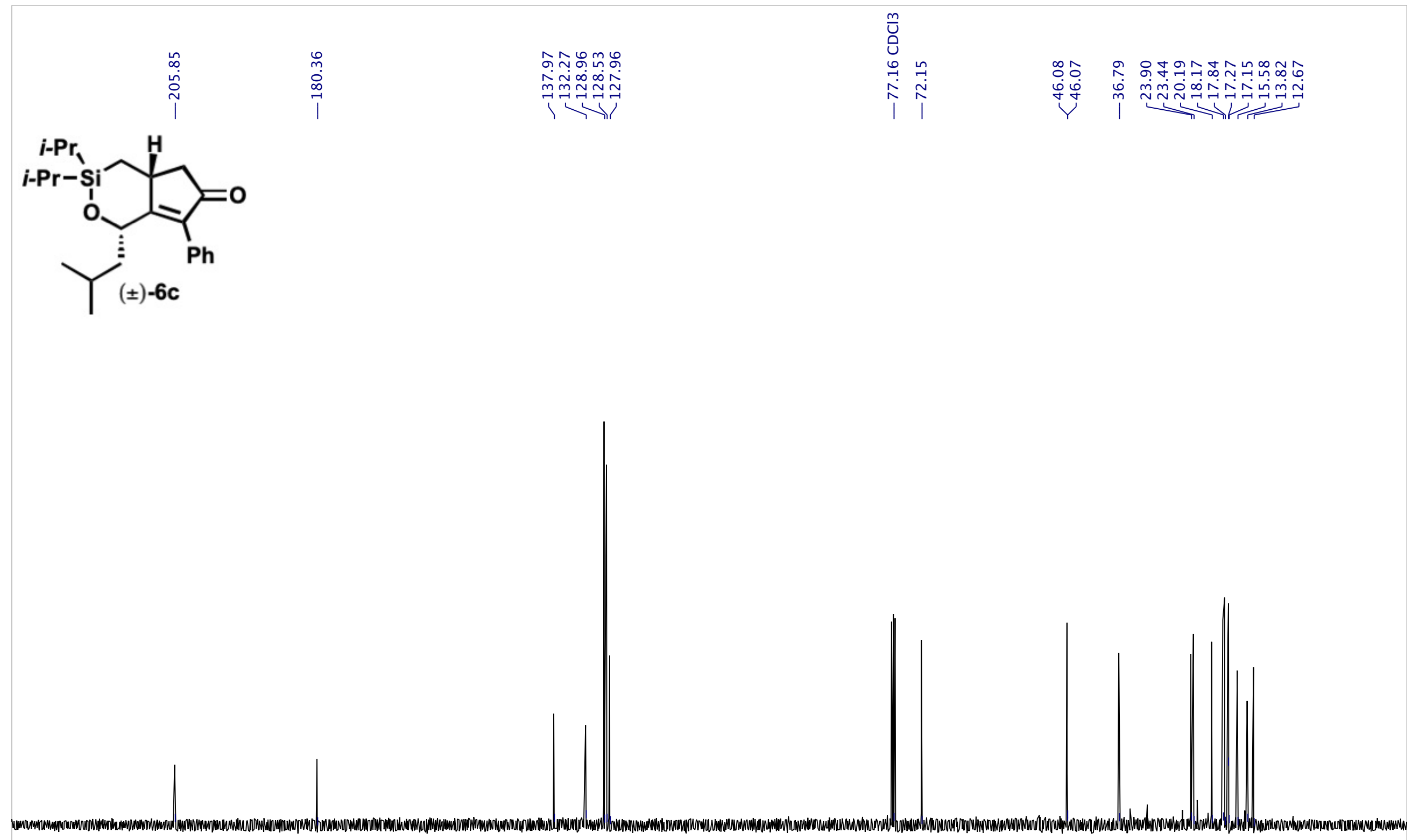

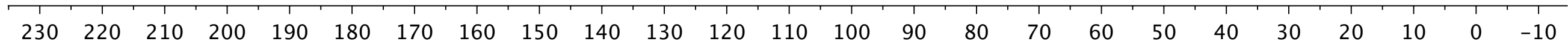




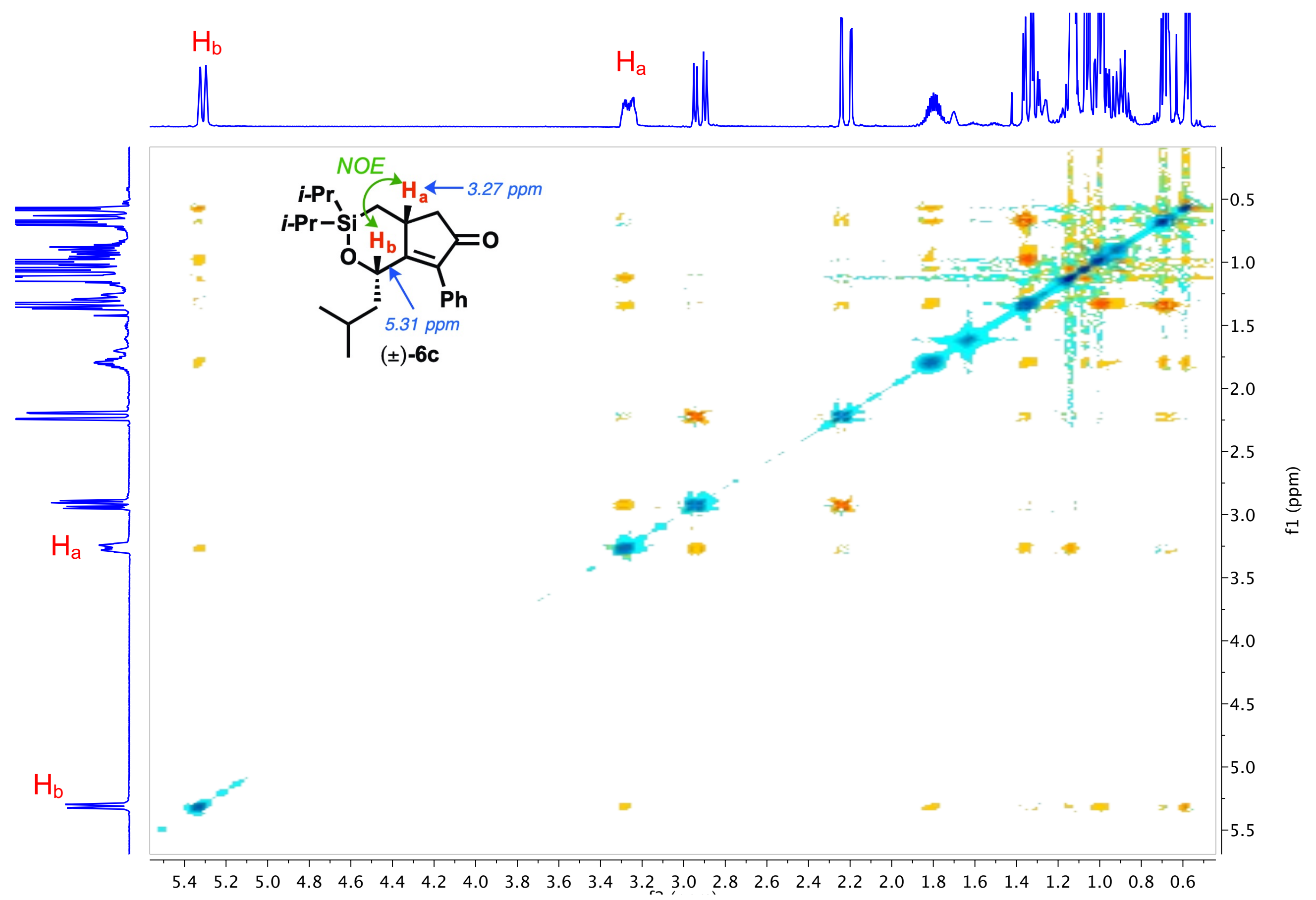




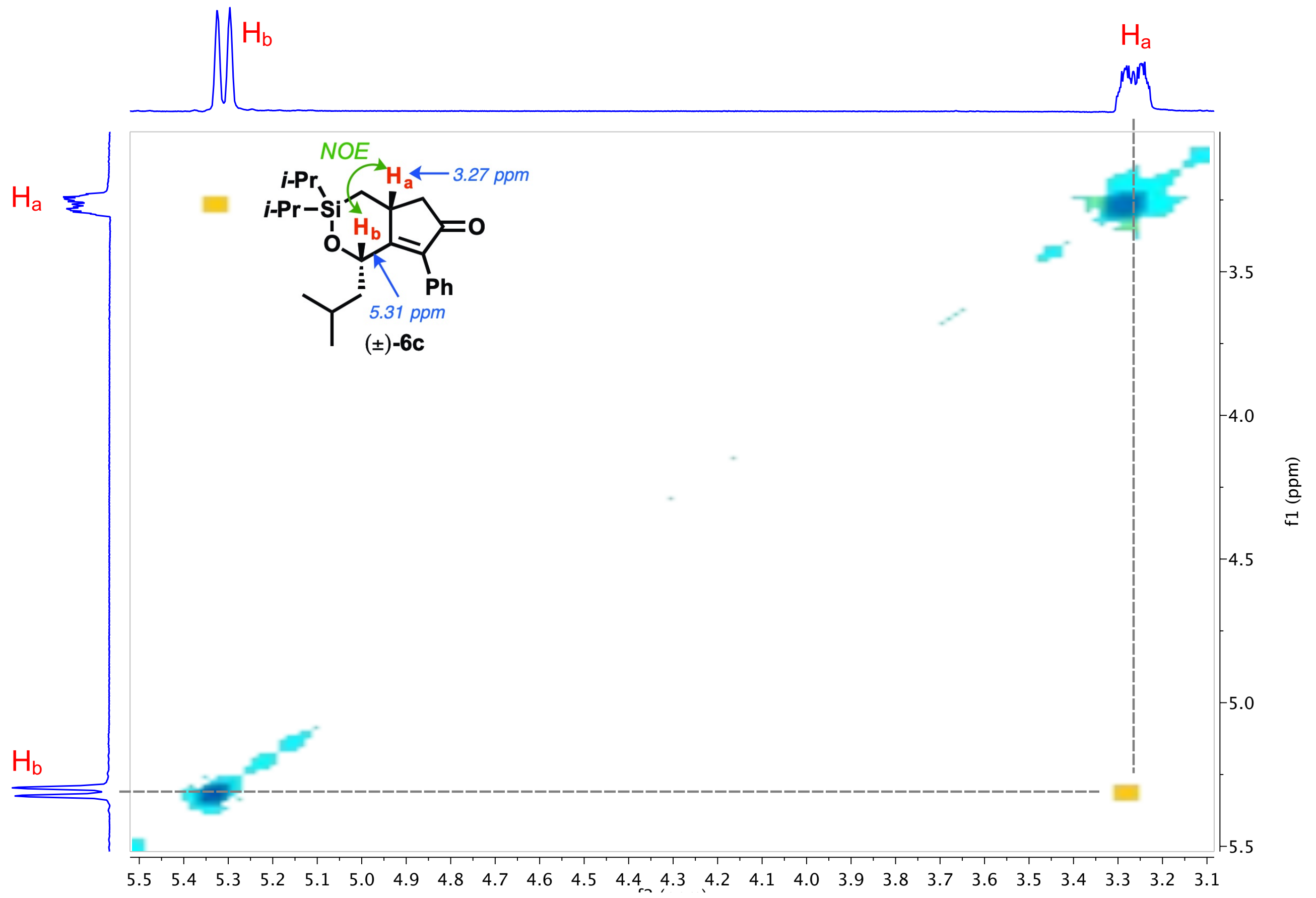




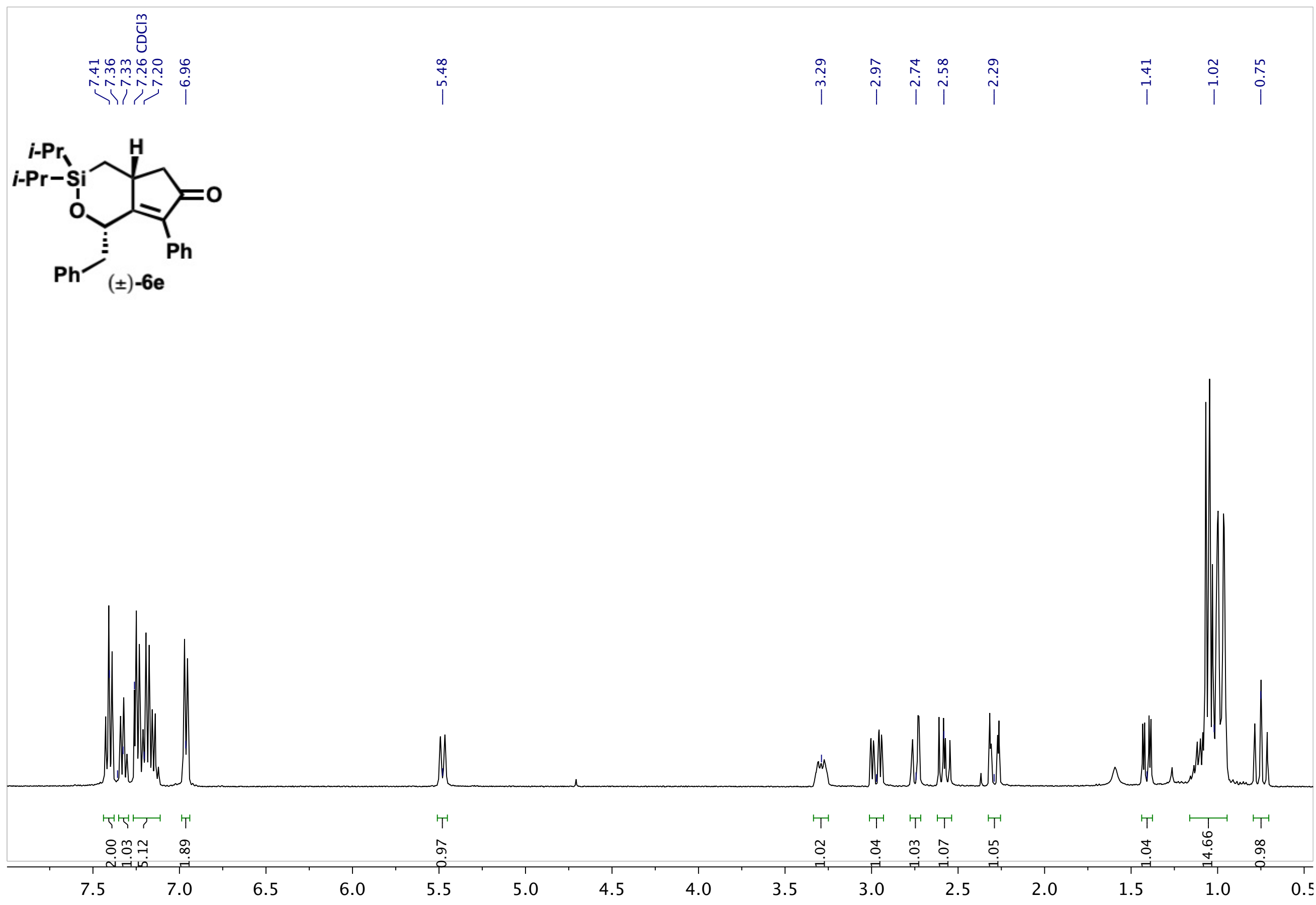




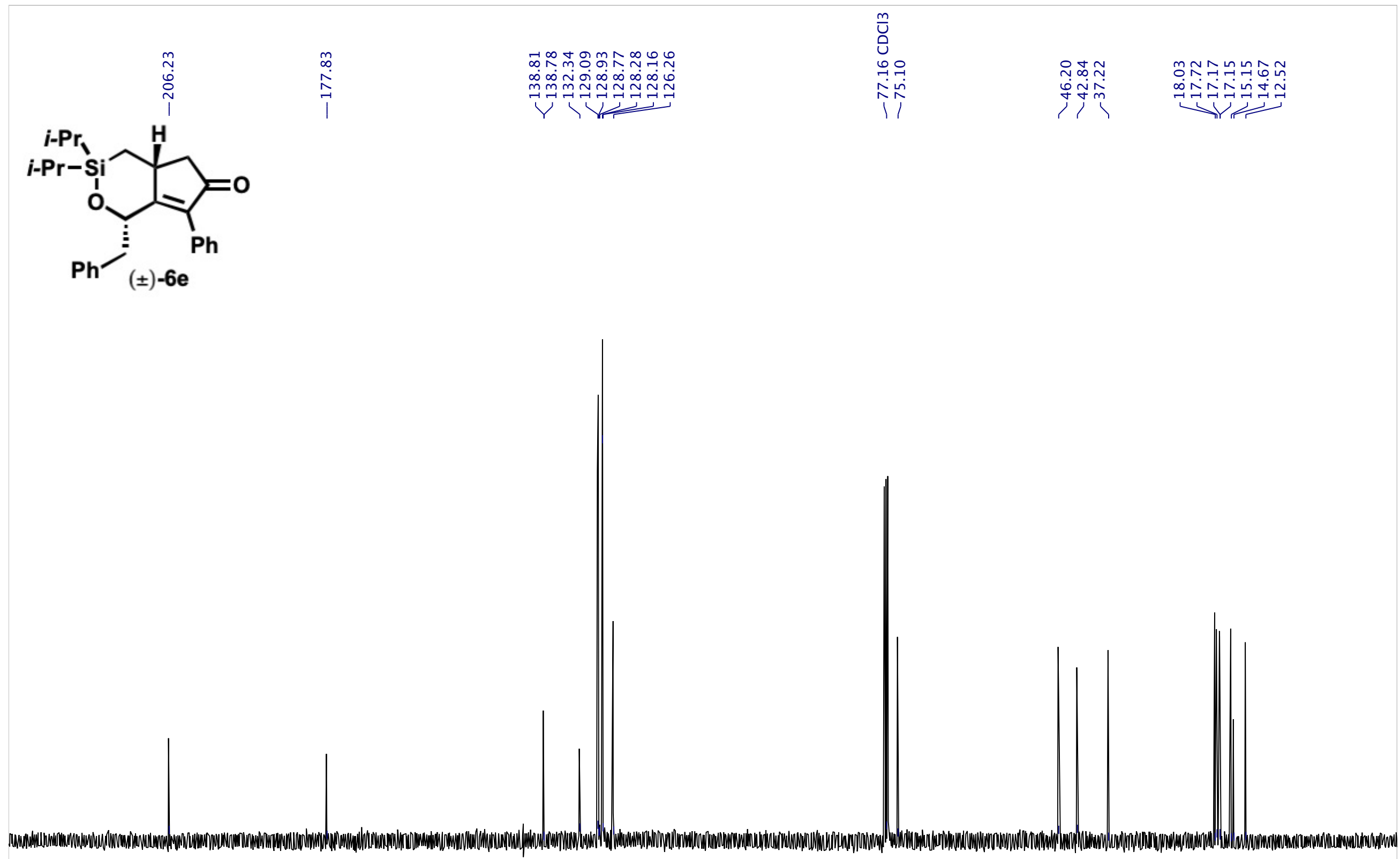

$\begin{array}{lllllllllllllllllllllllll}230 & 220 & 210 & 200 & 190 & 180 & 170 & 160 & 150 & 140 & 130 & 120 & 110 & 100 & 90 & 80 & 70 & 60 & 50 & 40 & 30 & 20 & 10 & 0 & -10\end{array}$




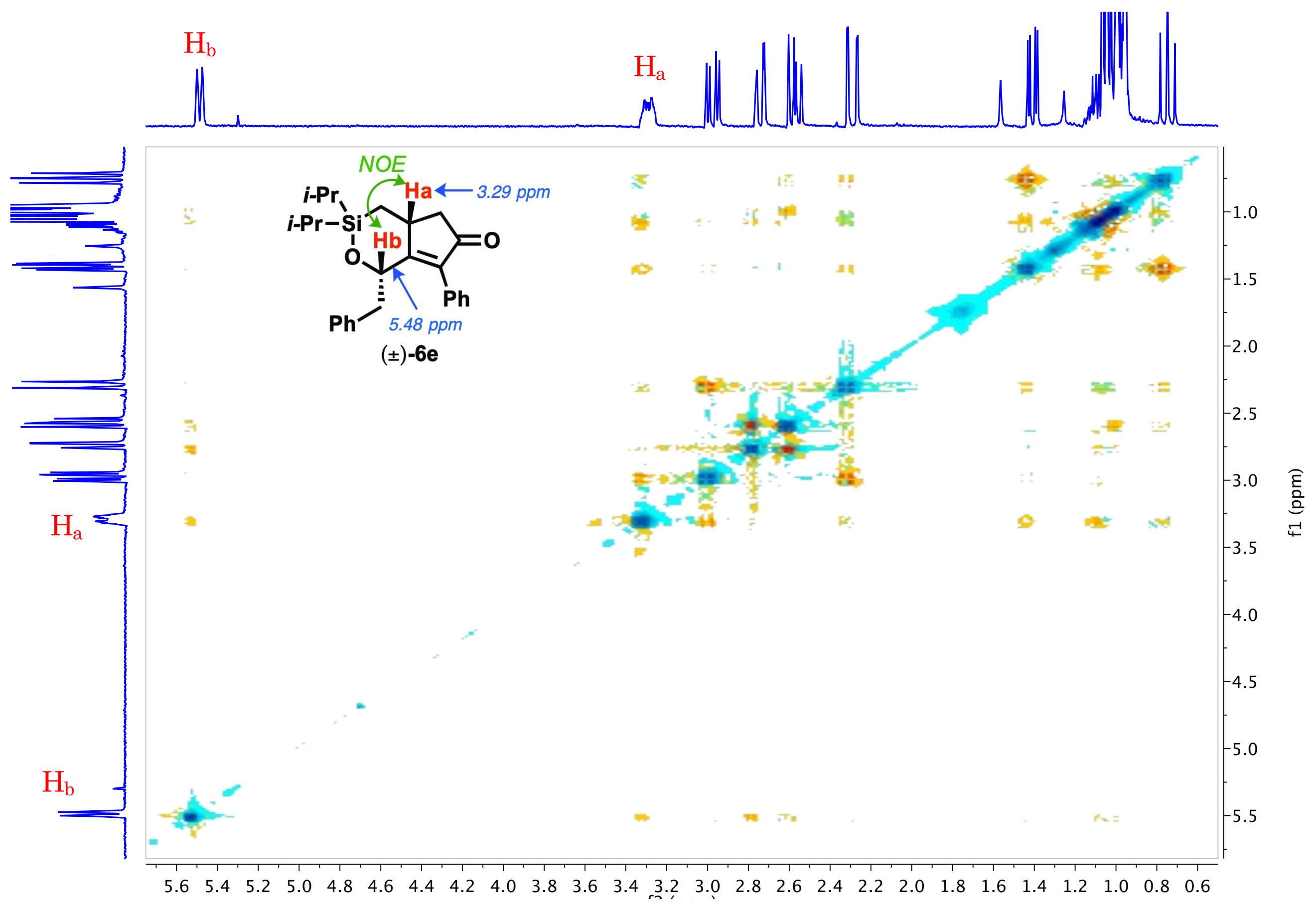




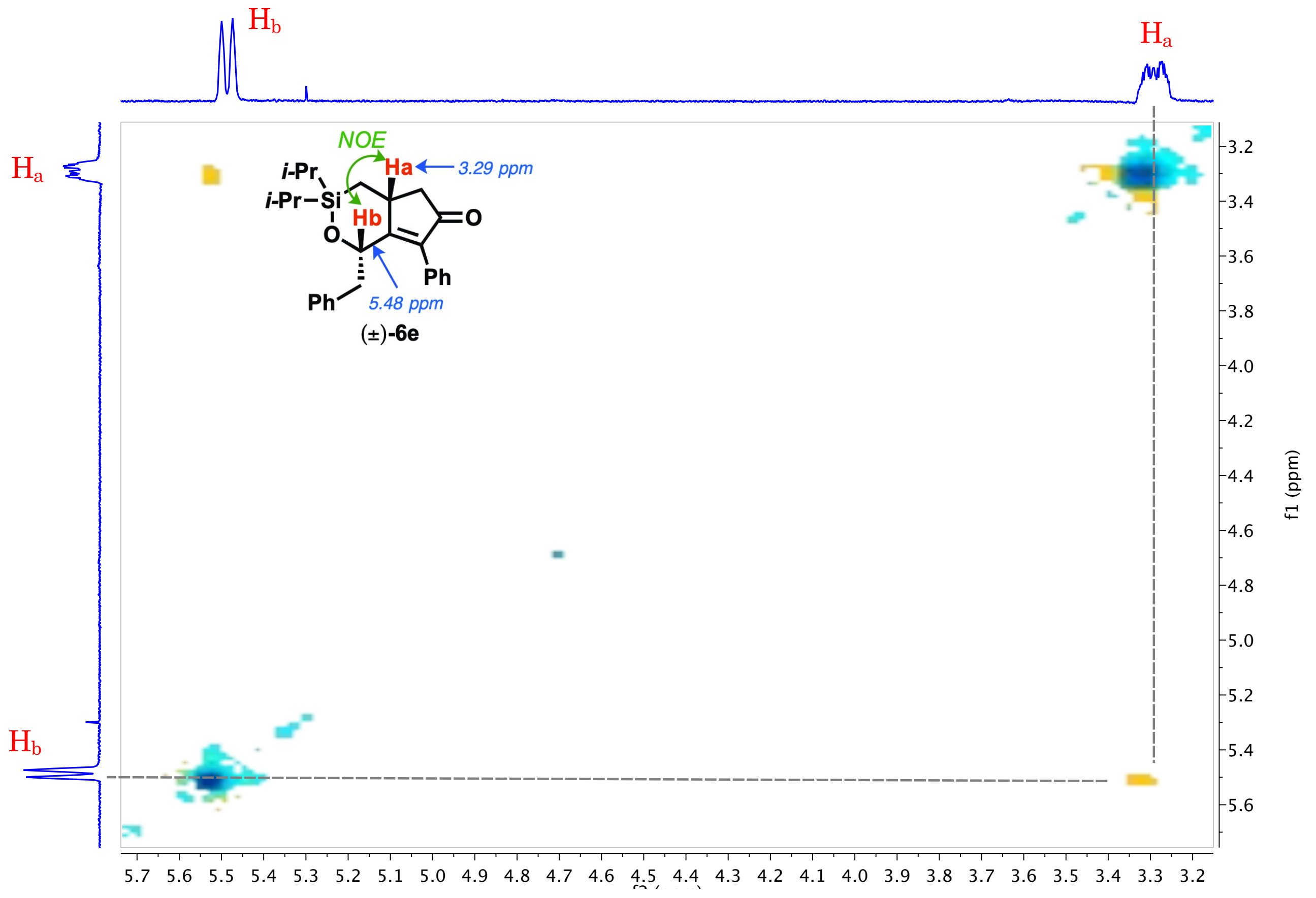



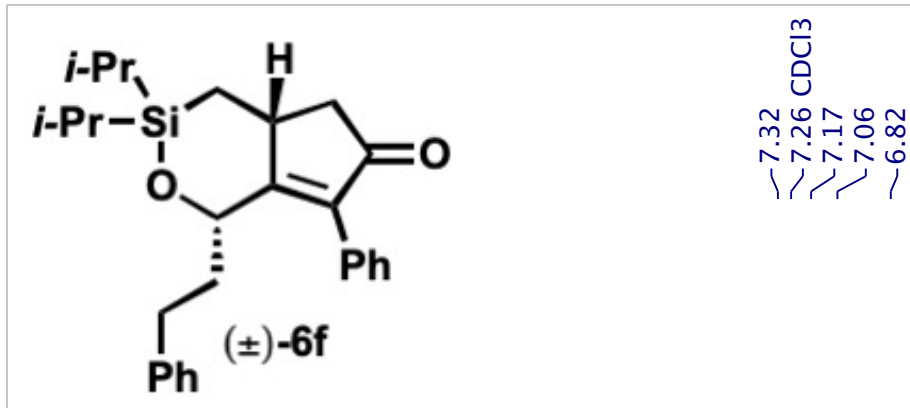

$\mathrm{Ph} \pm$

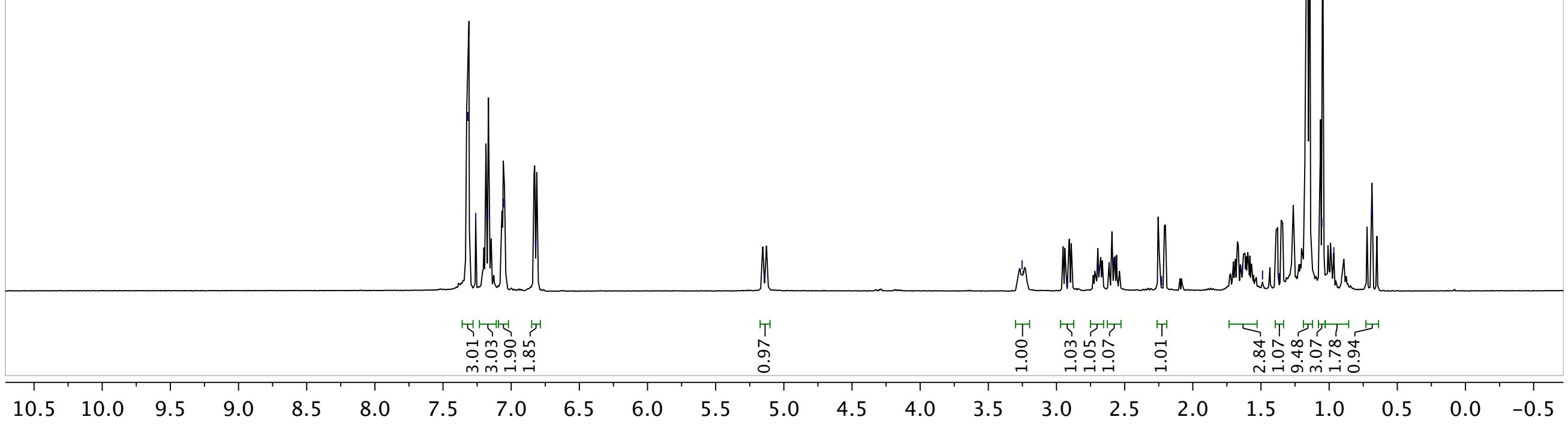




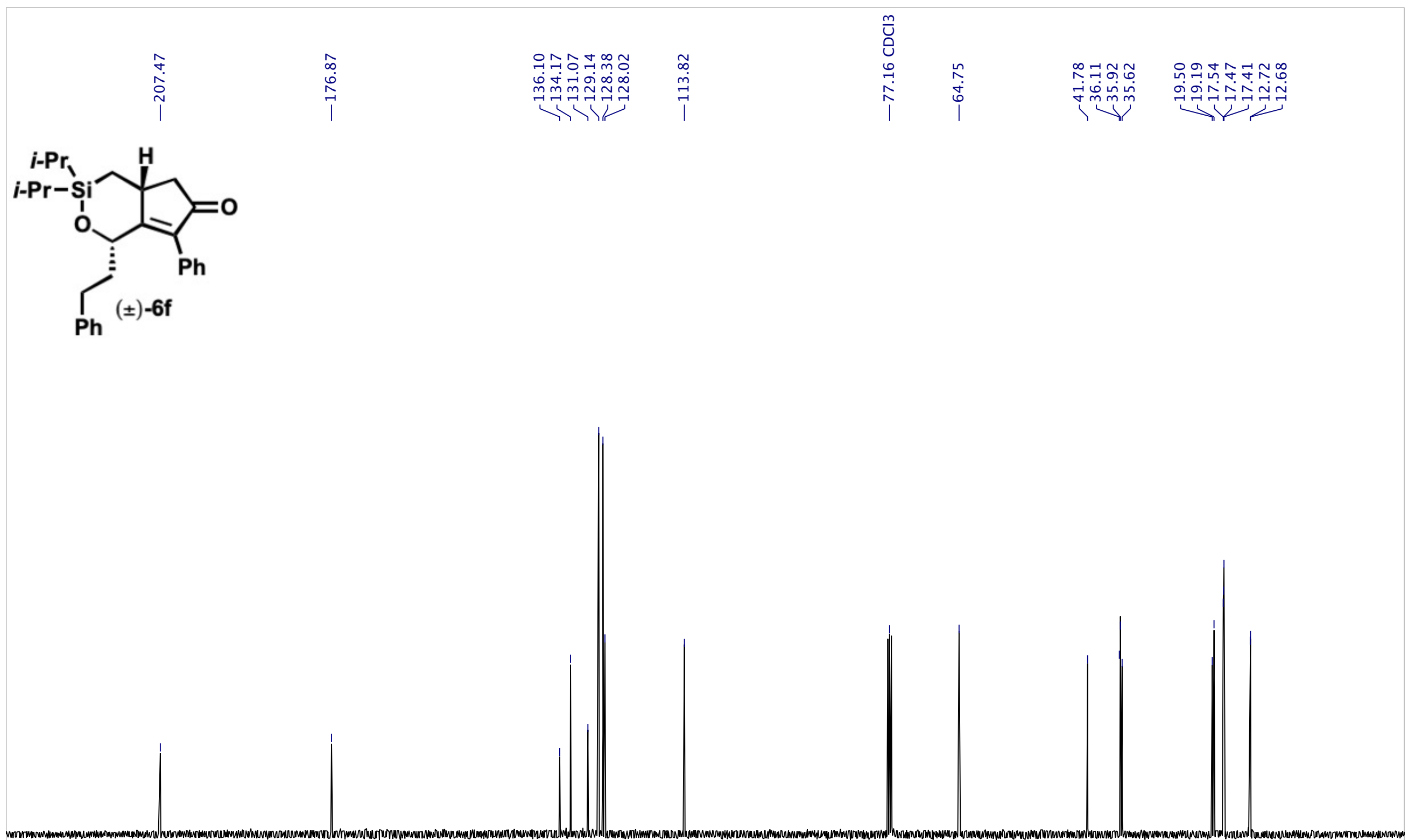

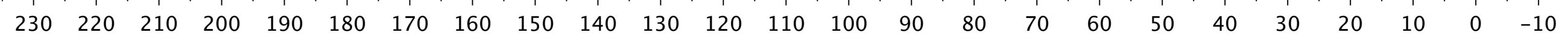




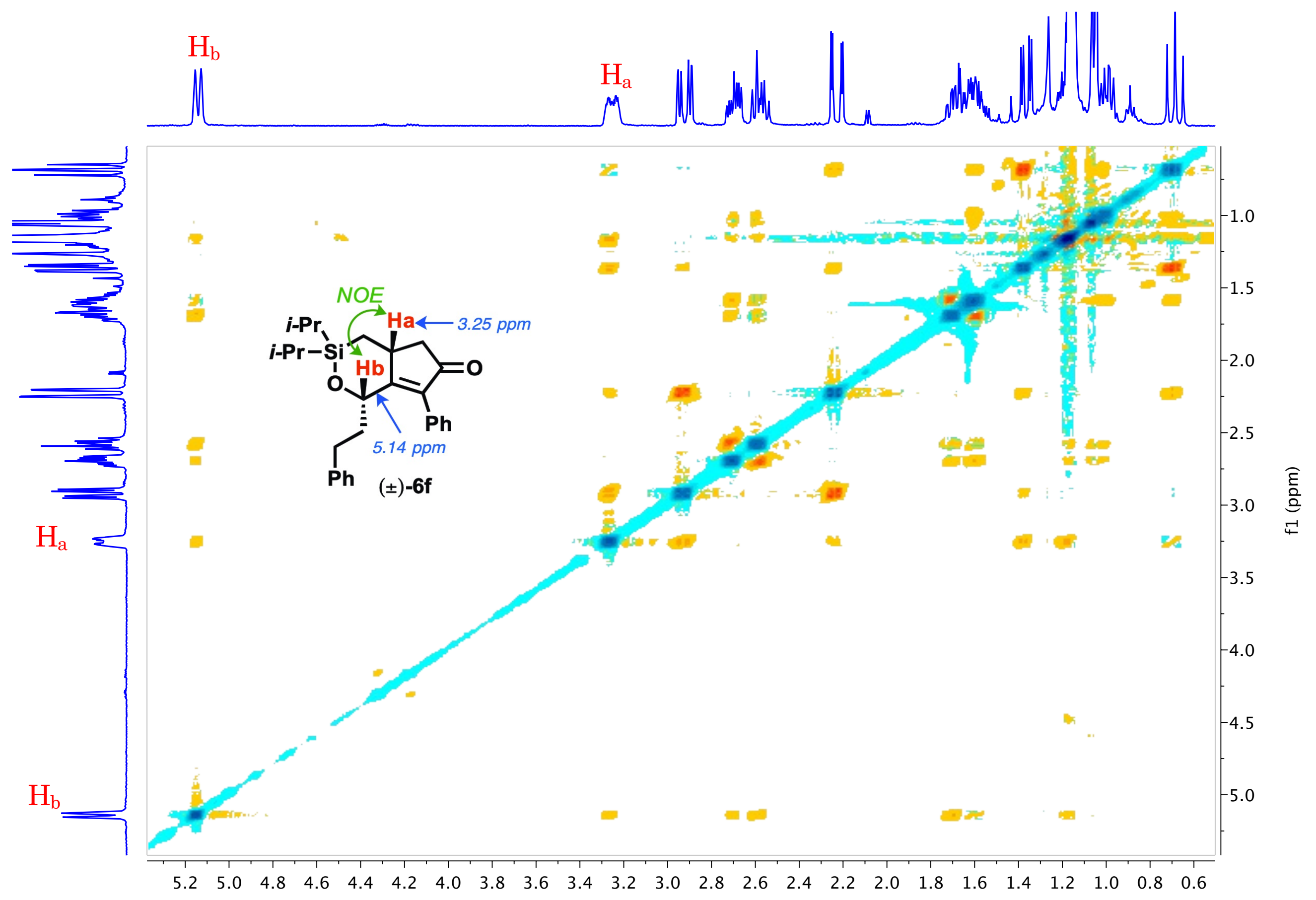




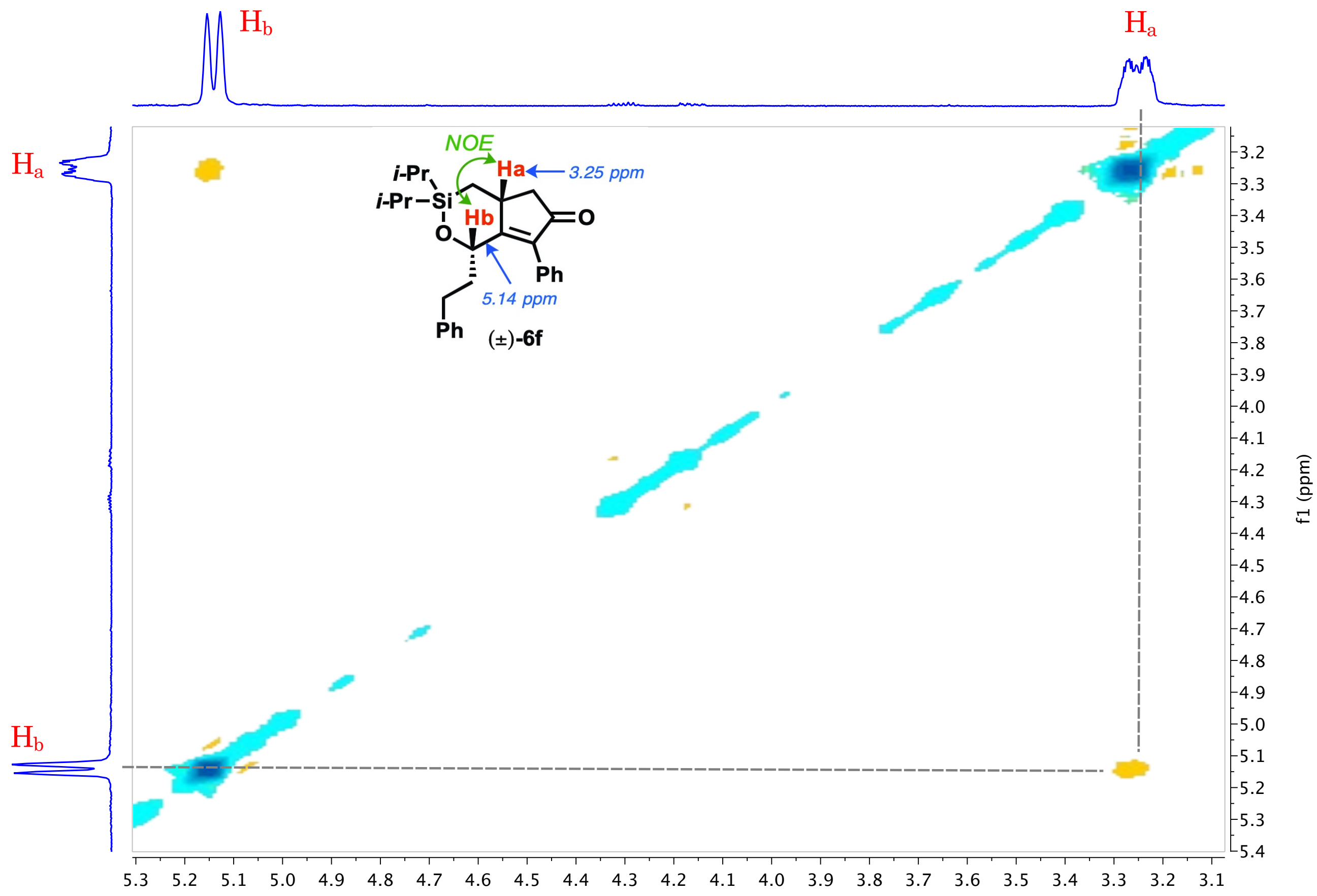




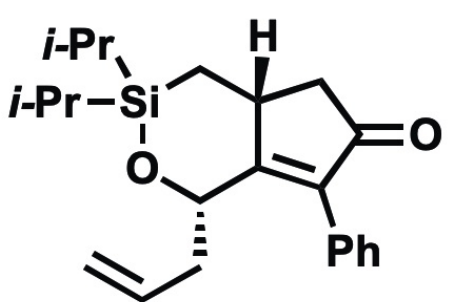

(士)-6g

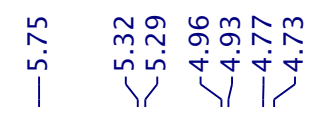

กัก คูำ

กุกั

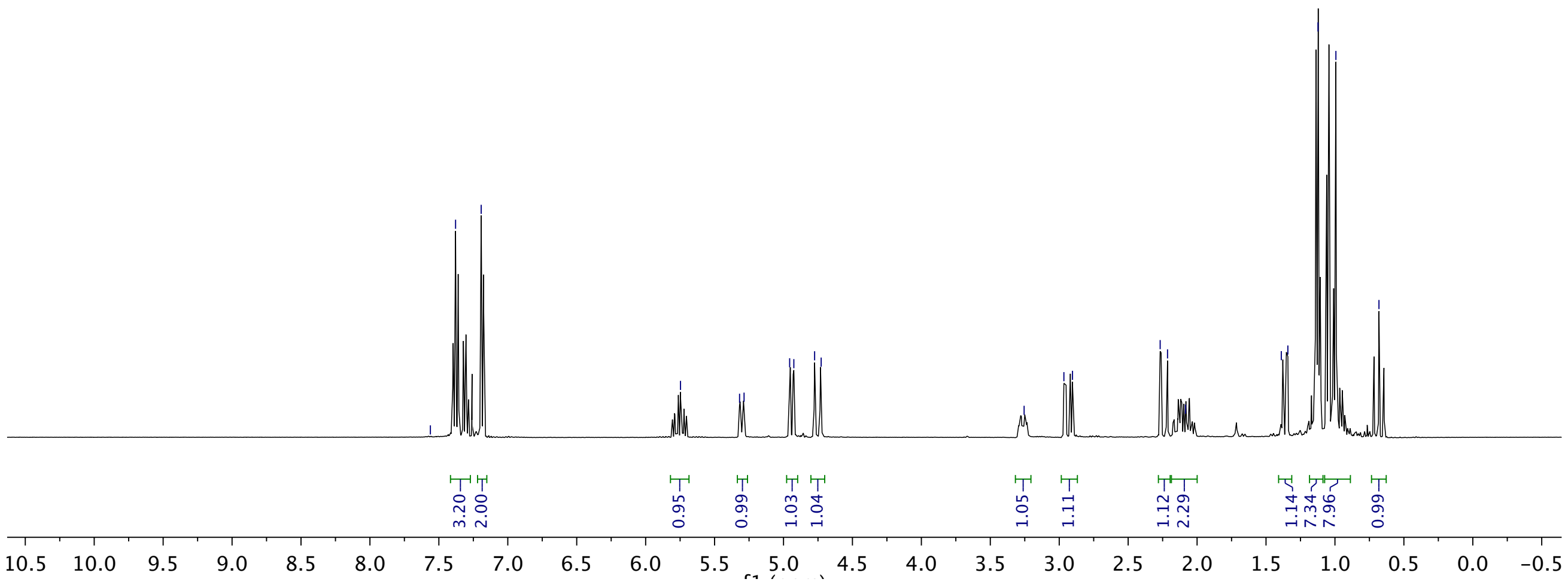




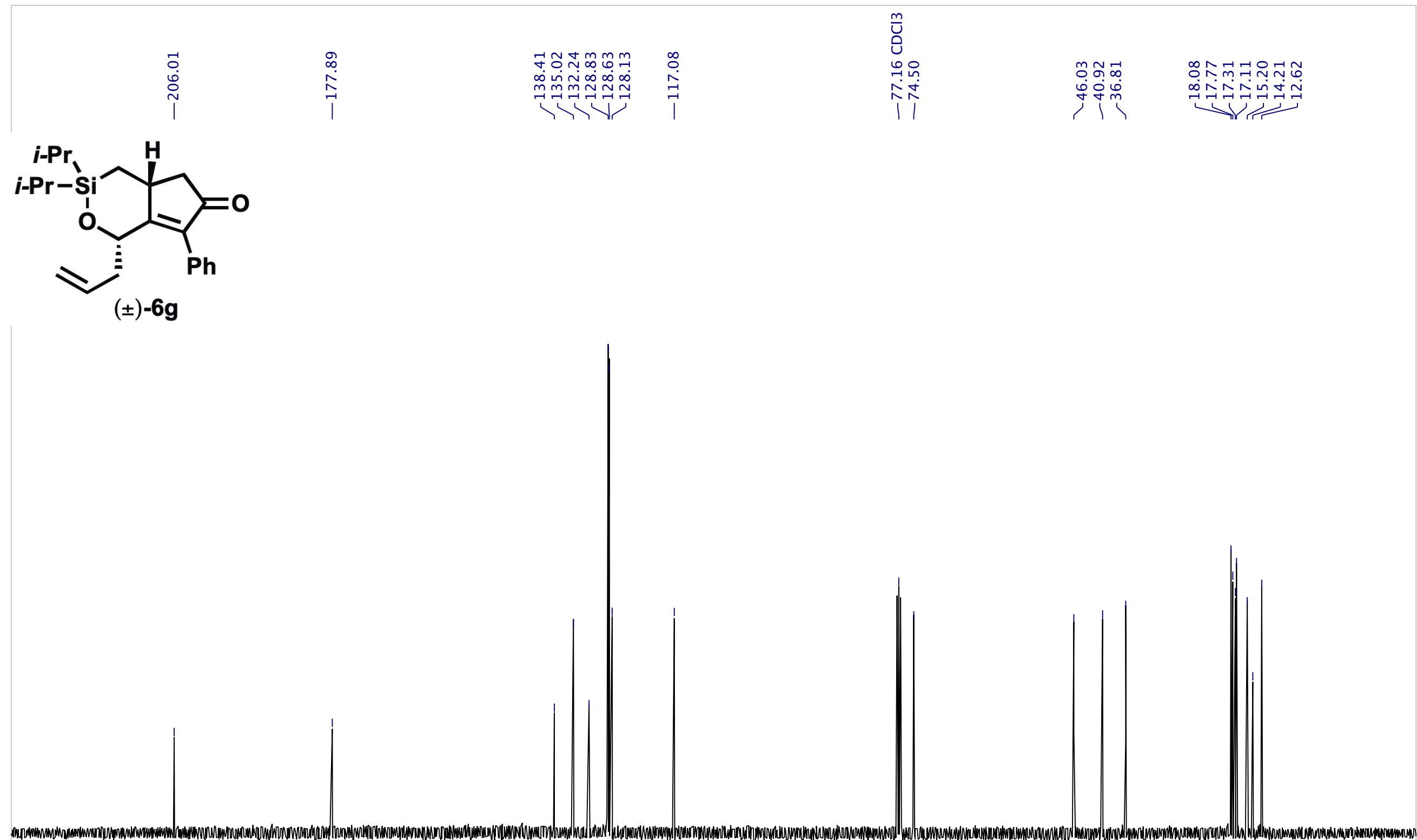

$\begin{array}{lllllllllllllllllllllllllll}230 & 220 & 210 & 200 & 190 & 180 & 170 & 160 & 150 & 140 & 130 & 120 & 110 & 100 & 90 & 80 & 70 & 60 & 50 & 40 & 30 & 20 & 10 & 0 & -10\end{array}$




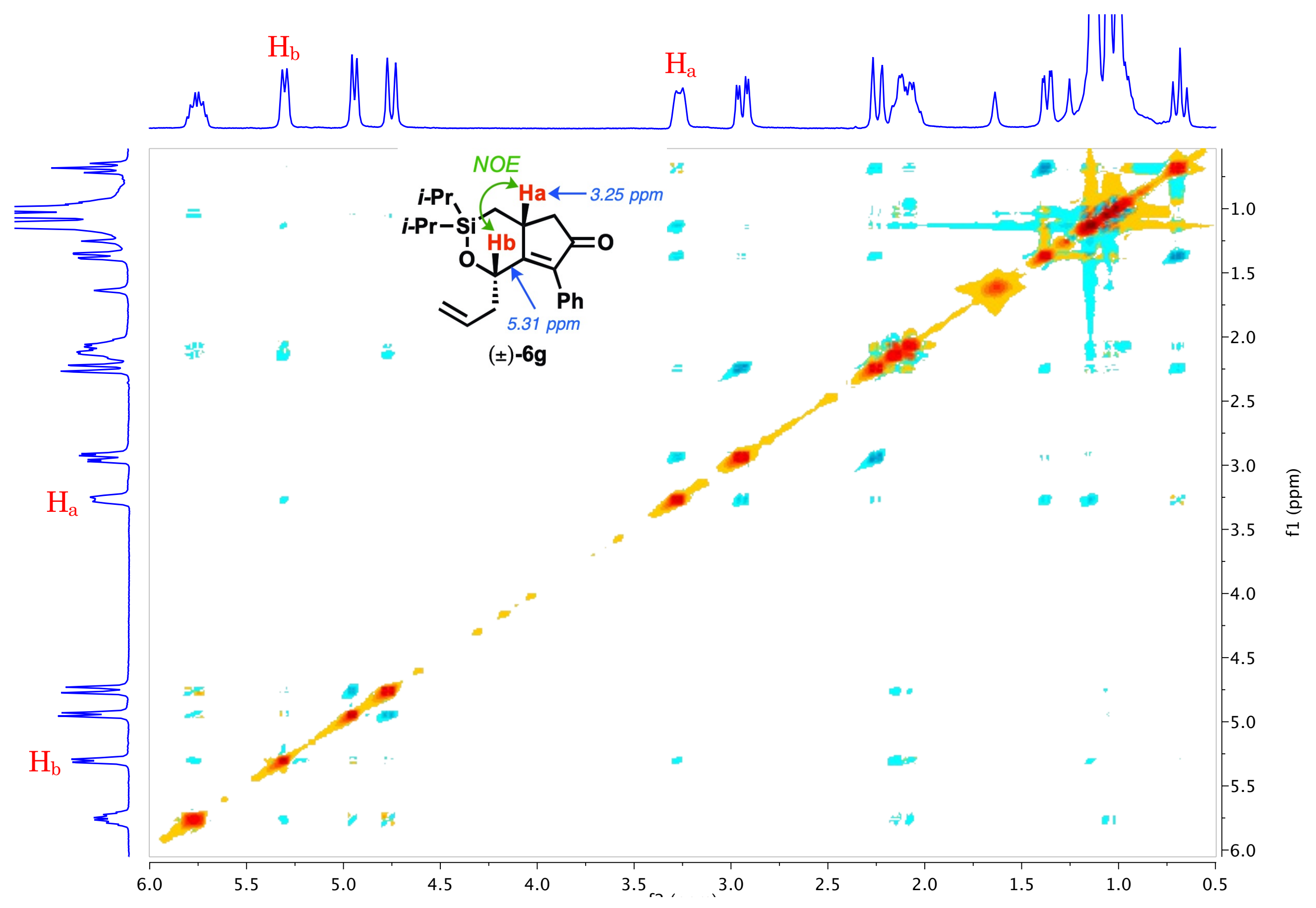




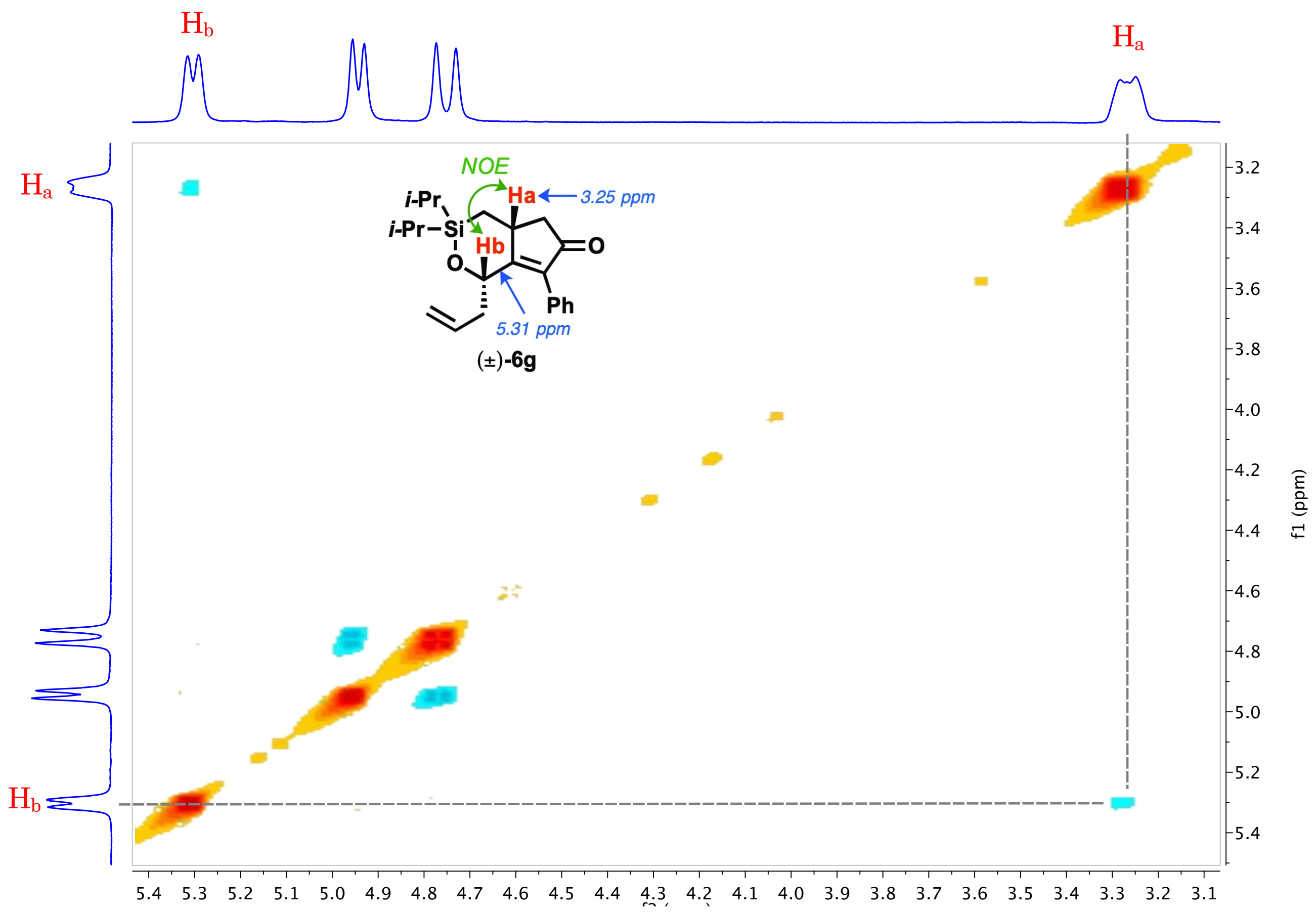




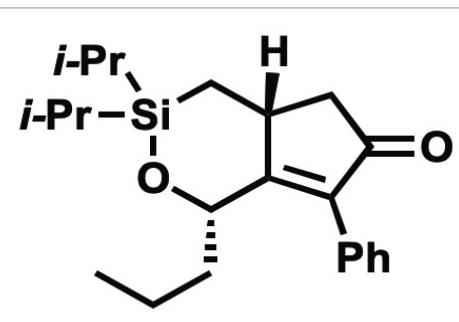

(土)-6h

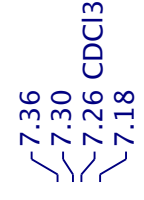

ตㄲำㅇํㅇํํ

itio

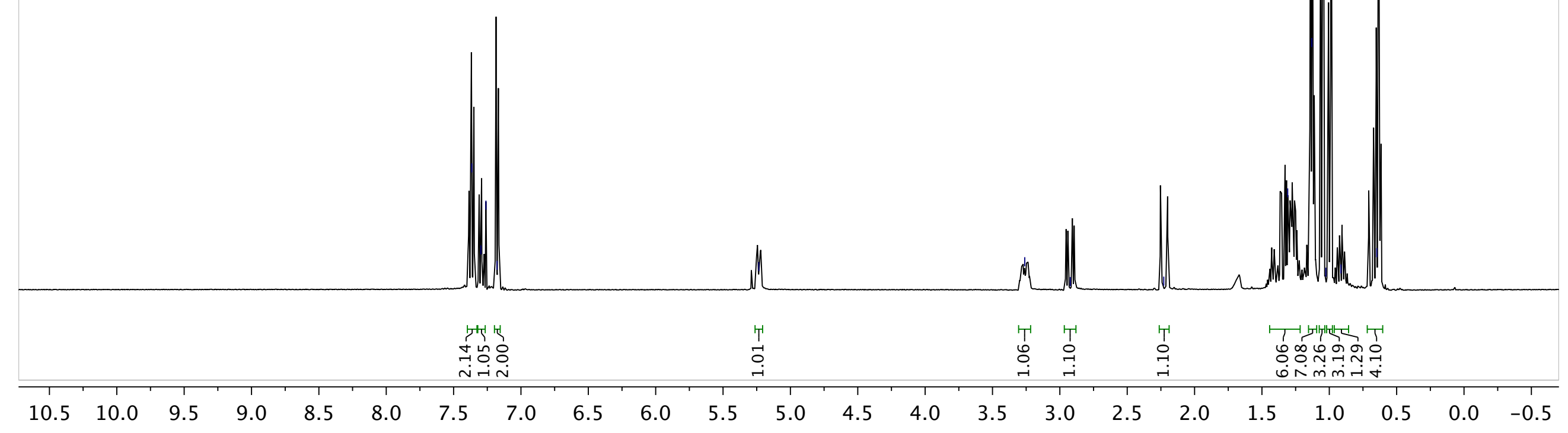




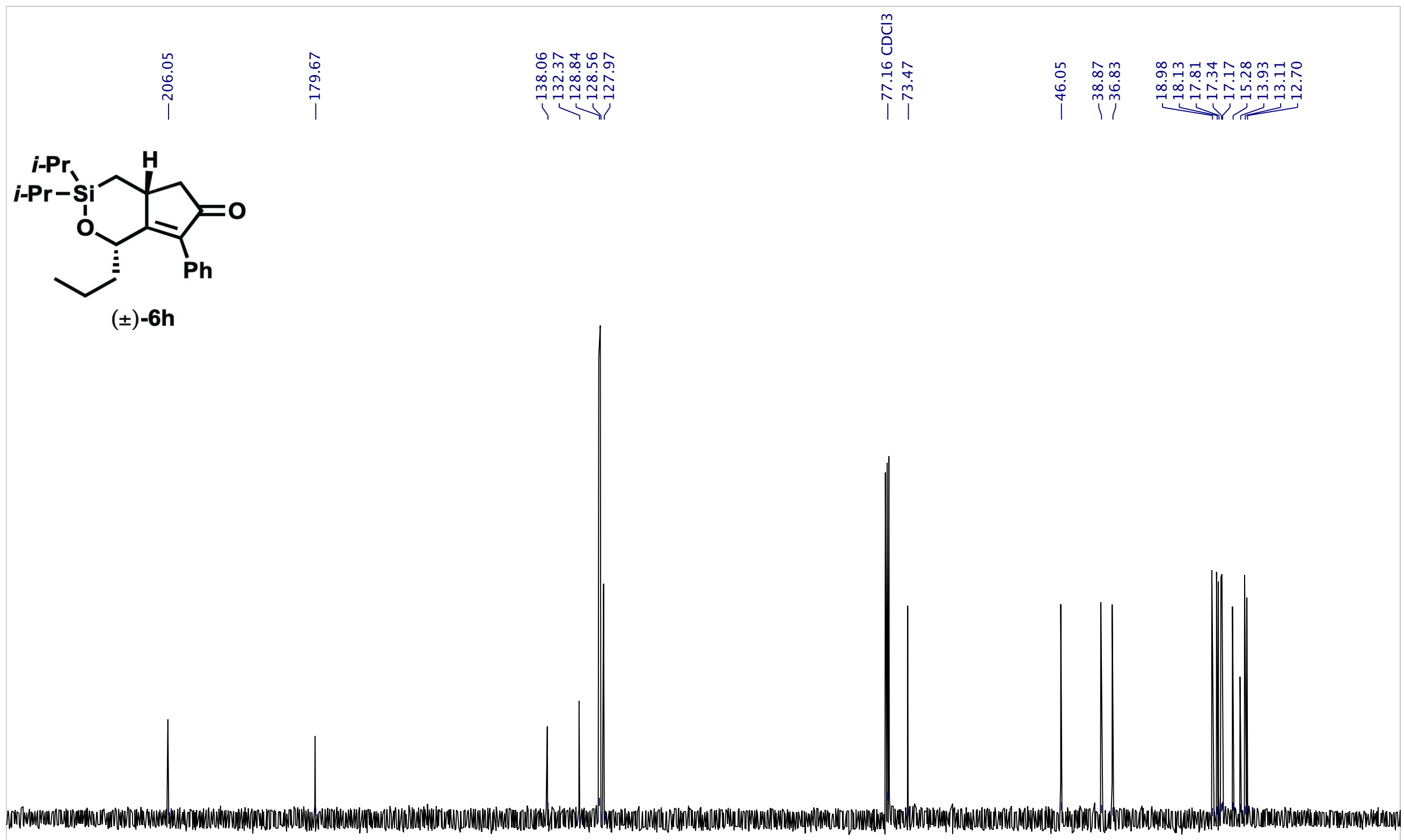

$\begin{array}{llllllllllllllllllllllllll}230 & 220 & 210 & 200 & 190 & 180 & 170 & 160 & 150 & 140 & 130 & 120 & 110 & 100 & 90 & 80 & 70 & 60 & 50 & 40 & 30 & 20 & 10 & 0 & -10\end{array}$




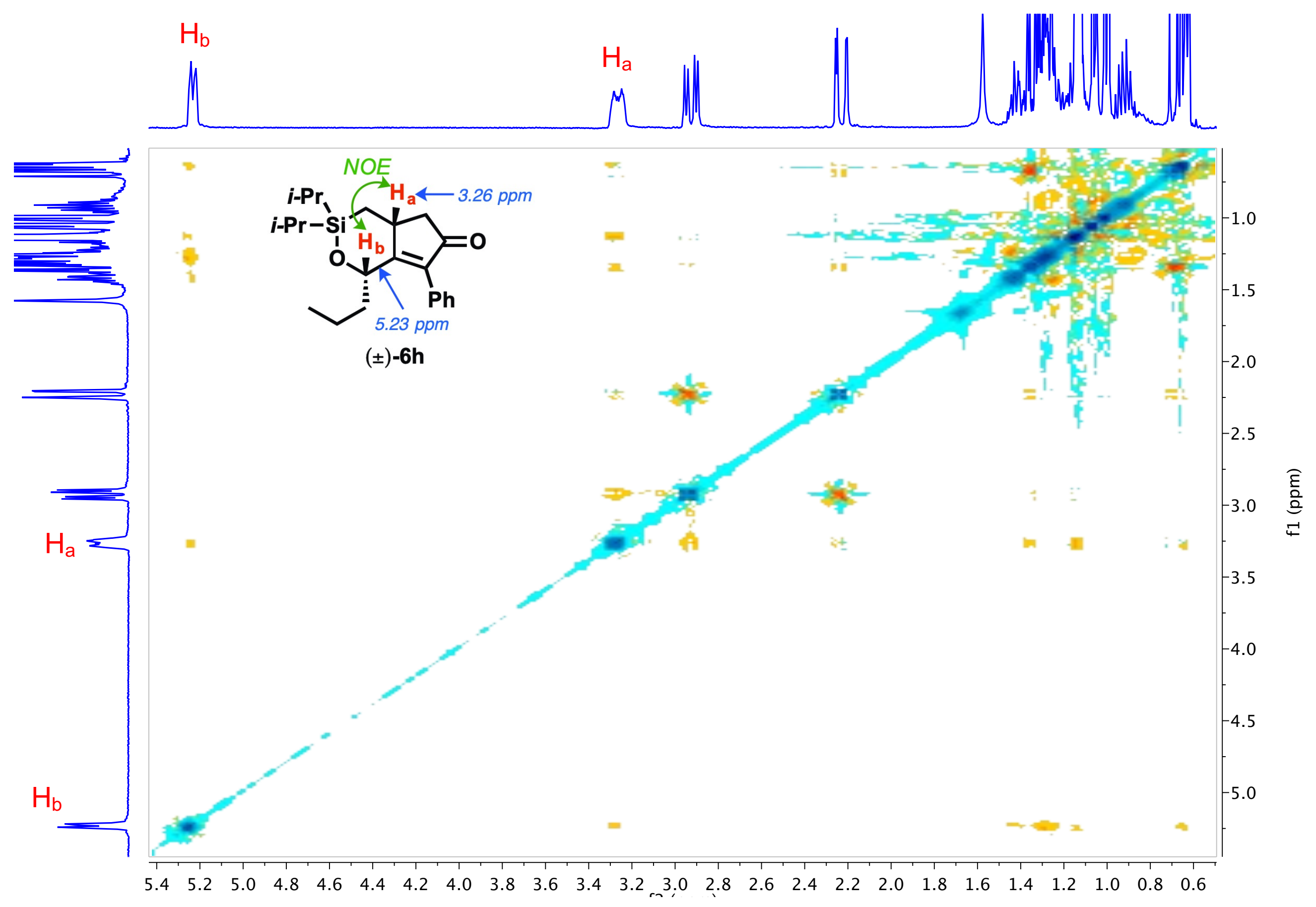




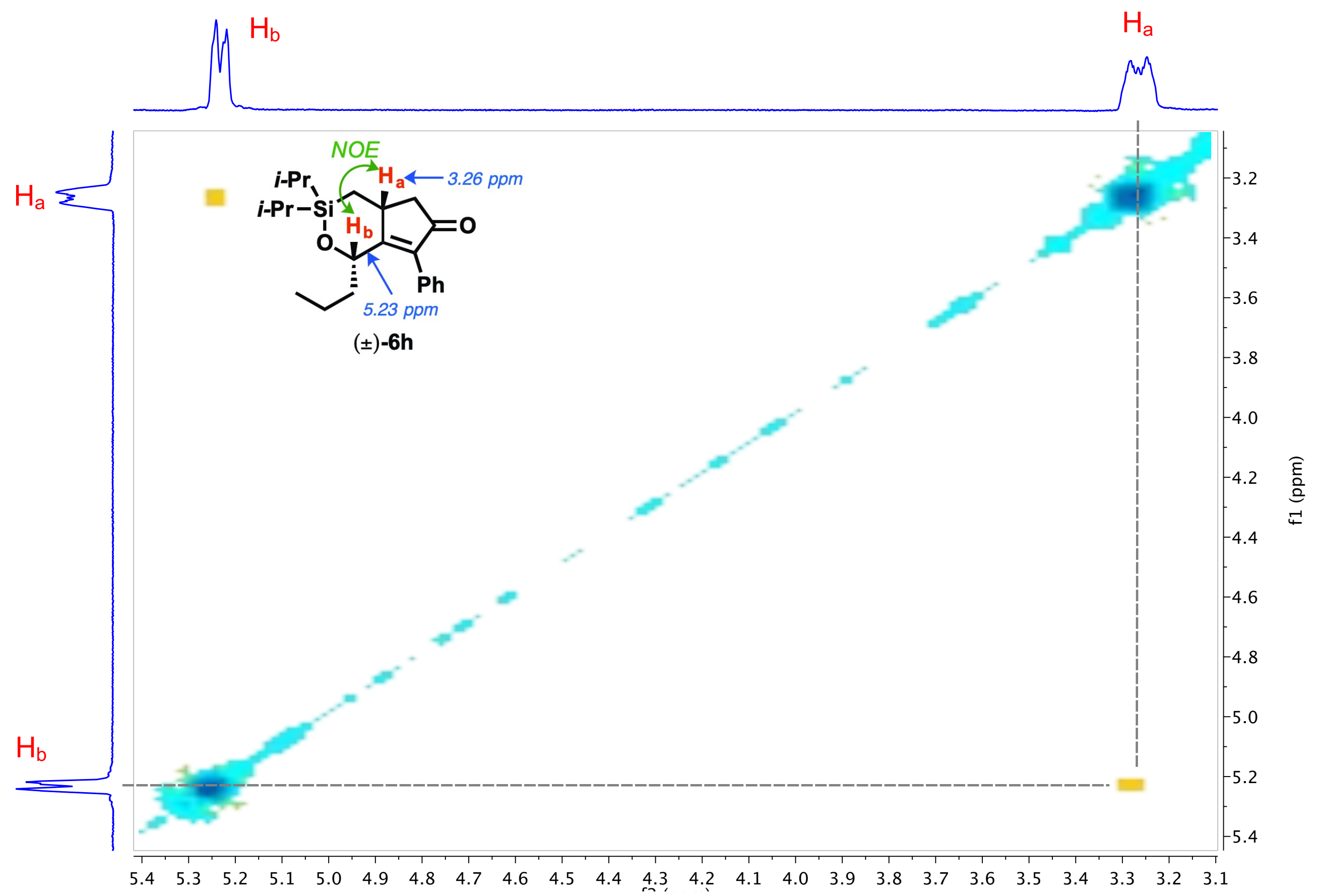




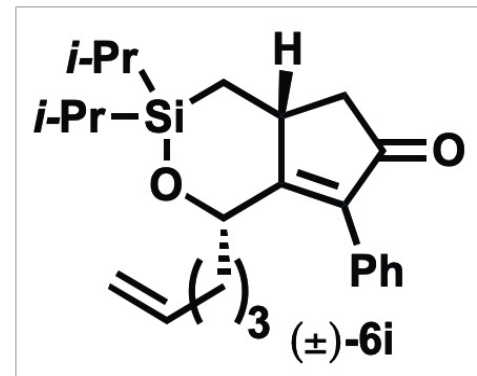

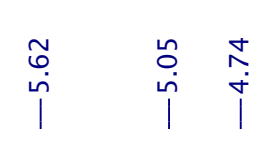

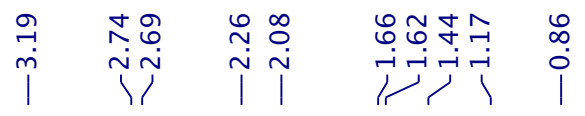

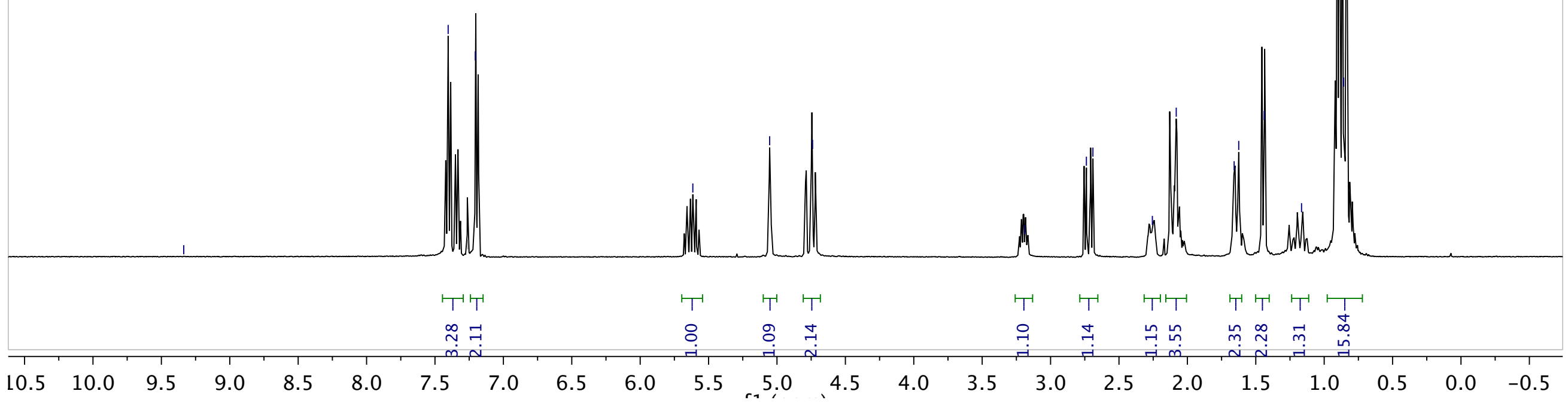




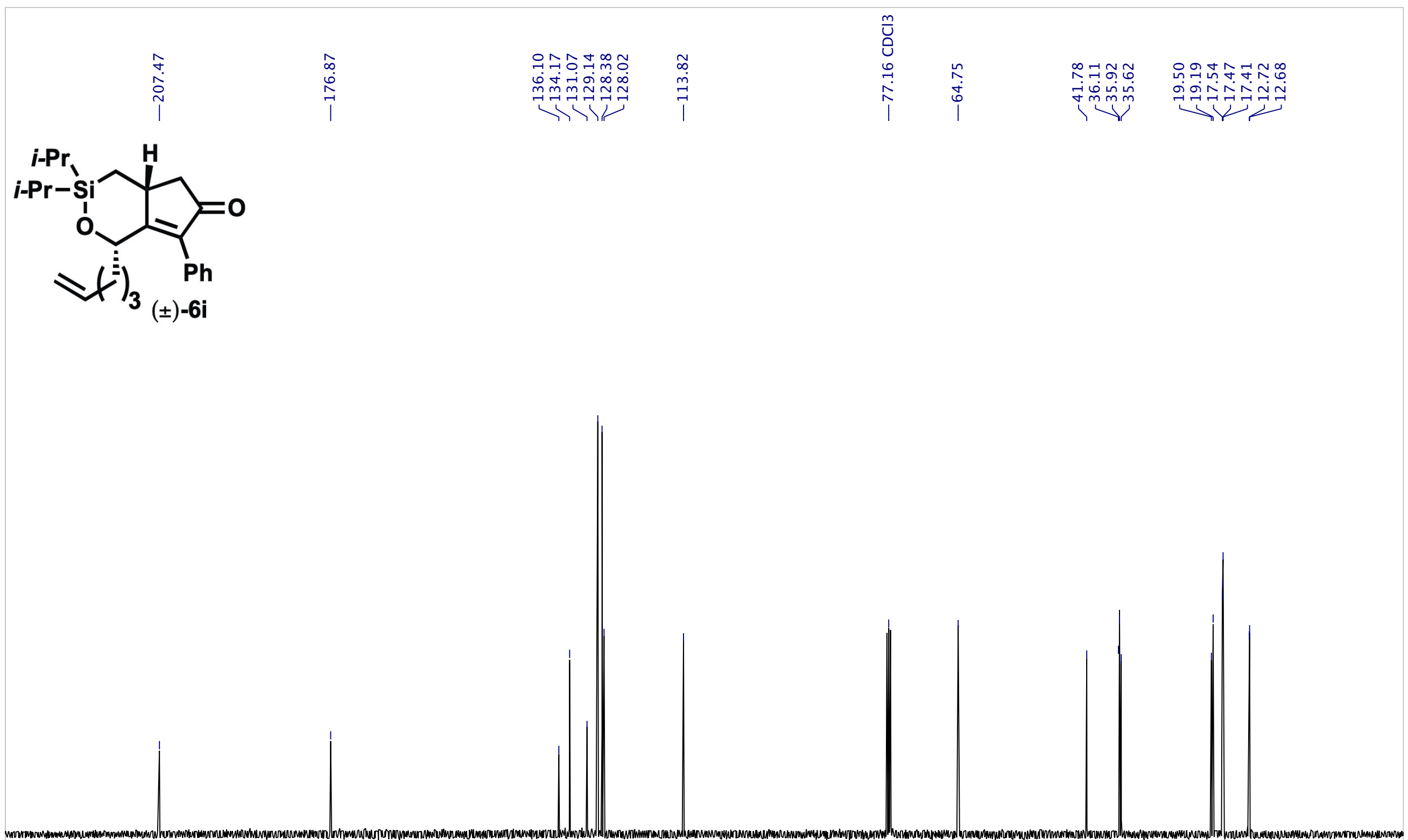

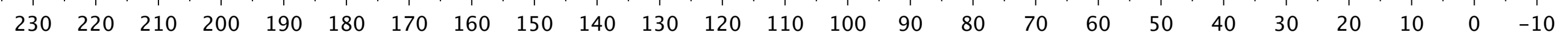




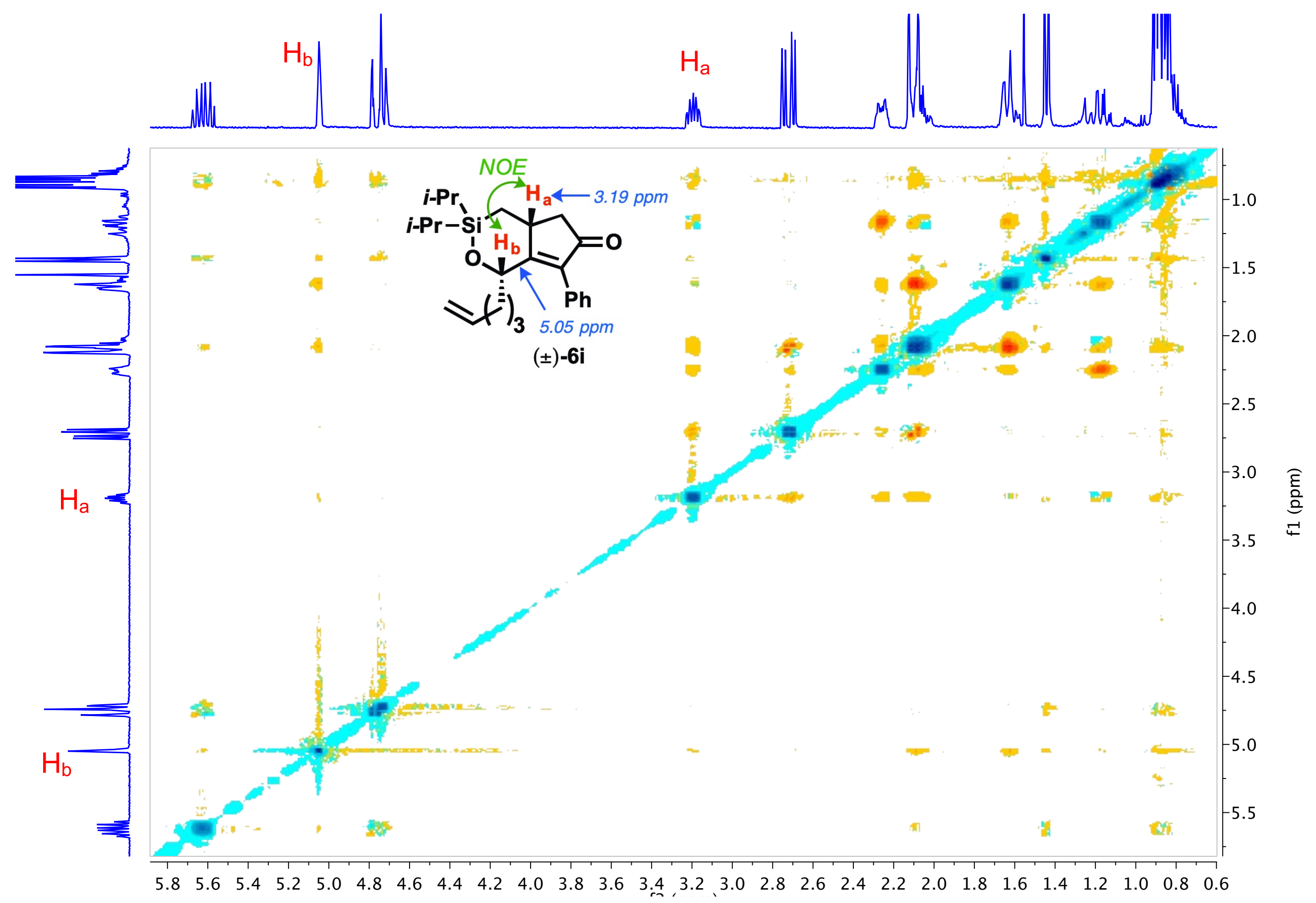




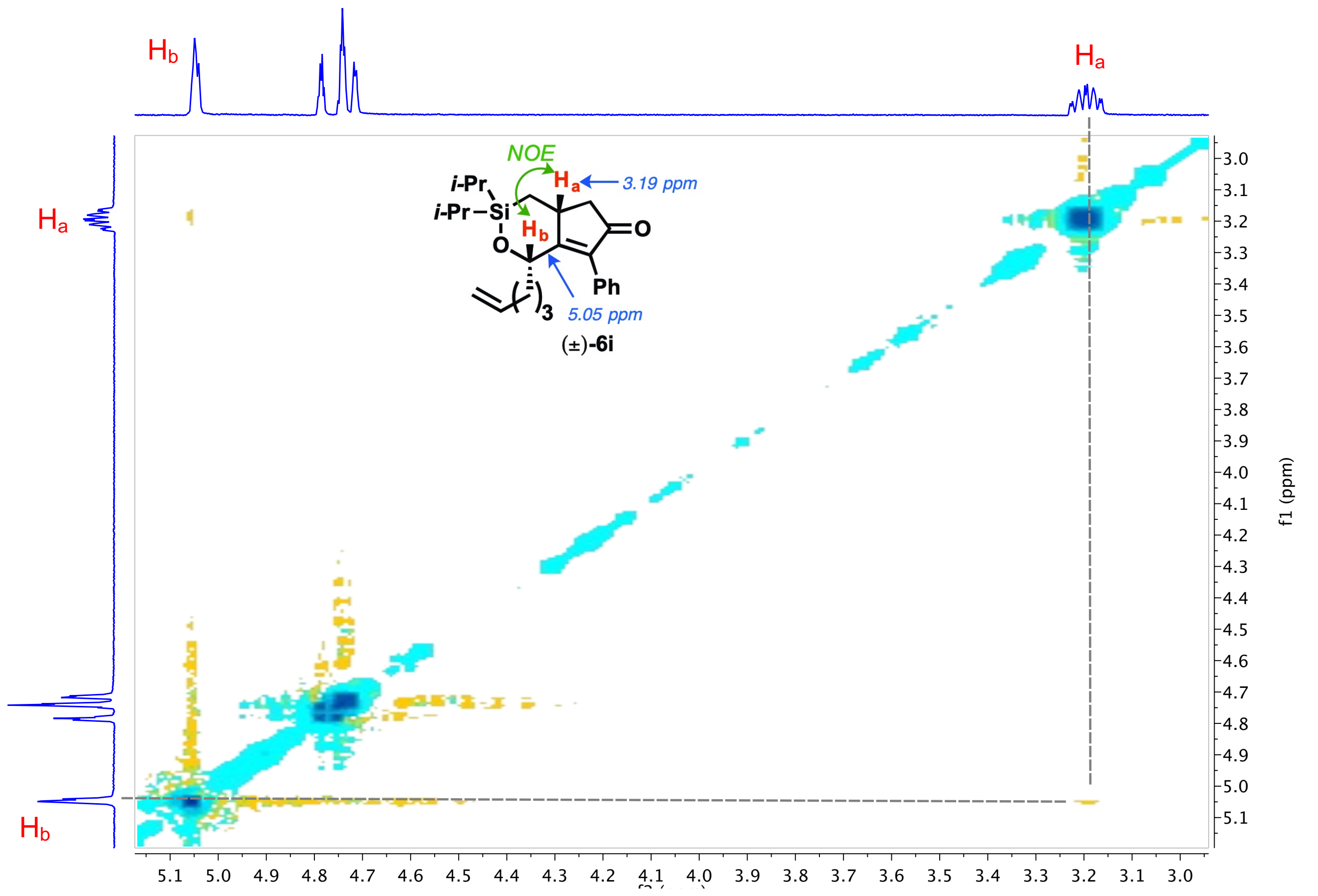



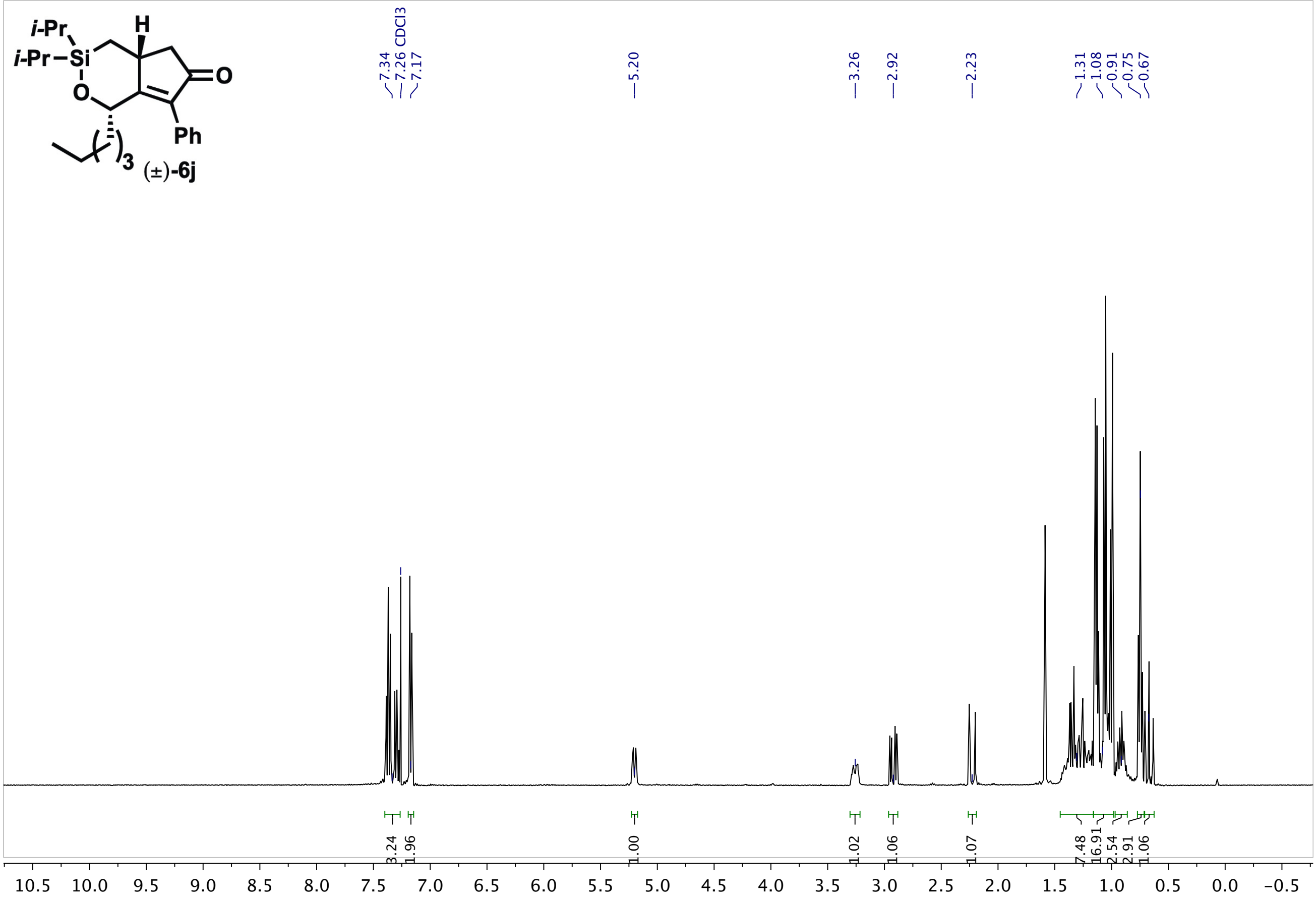


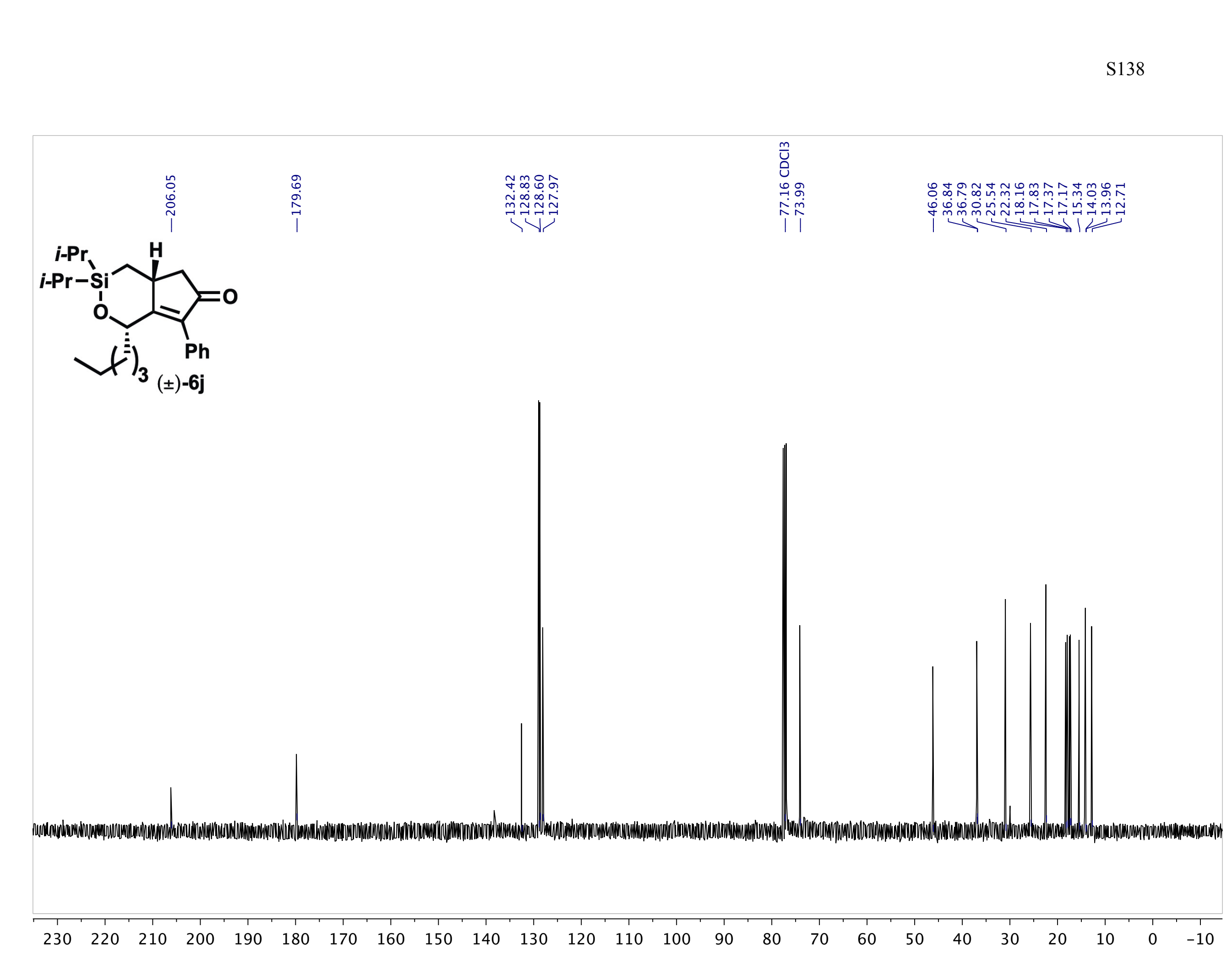




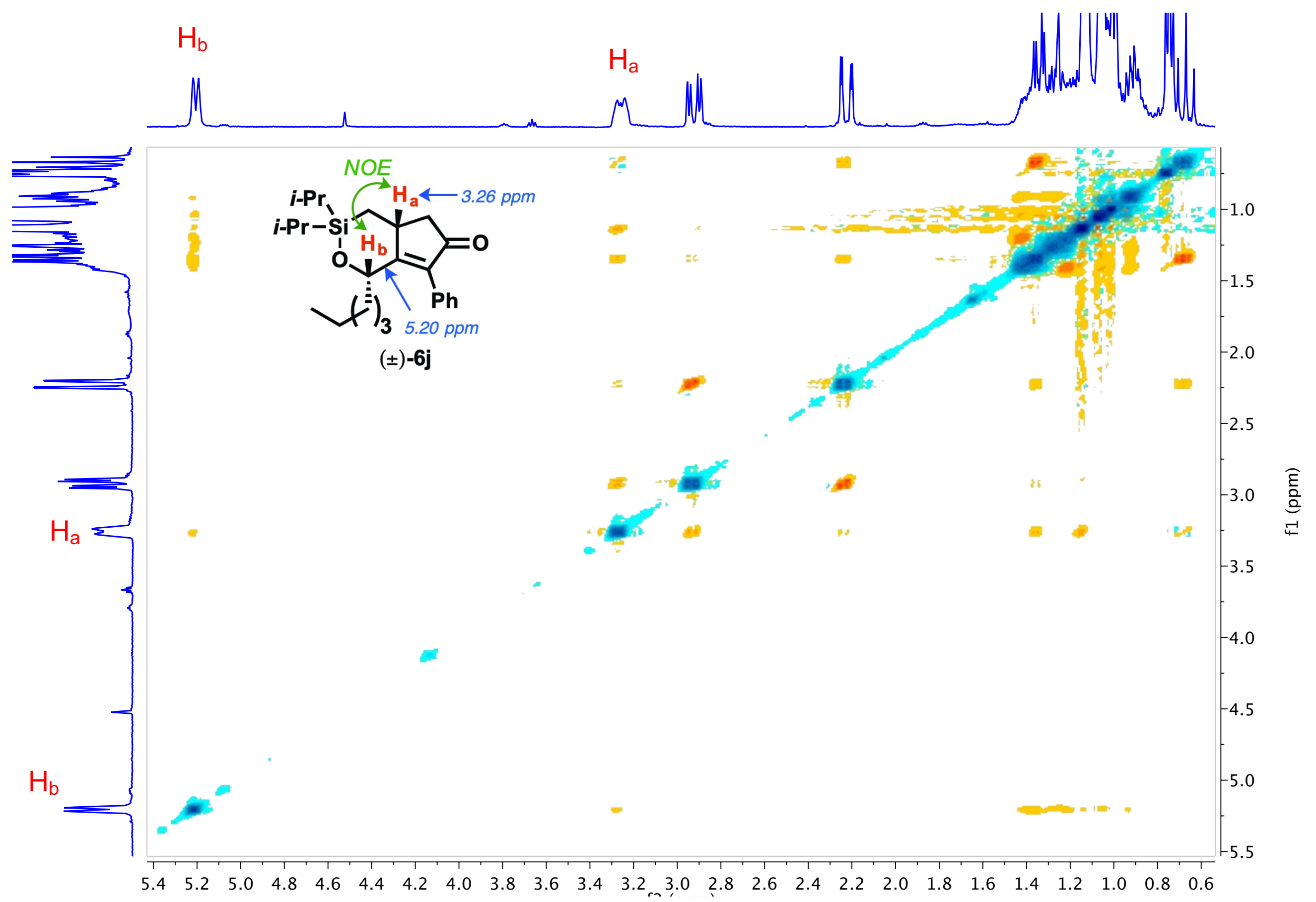




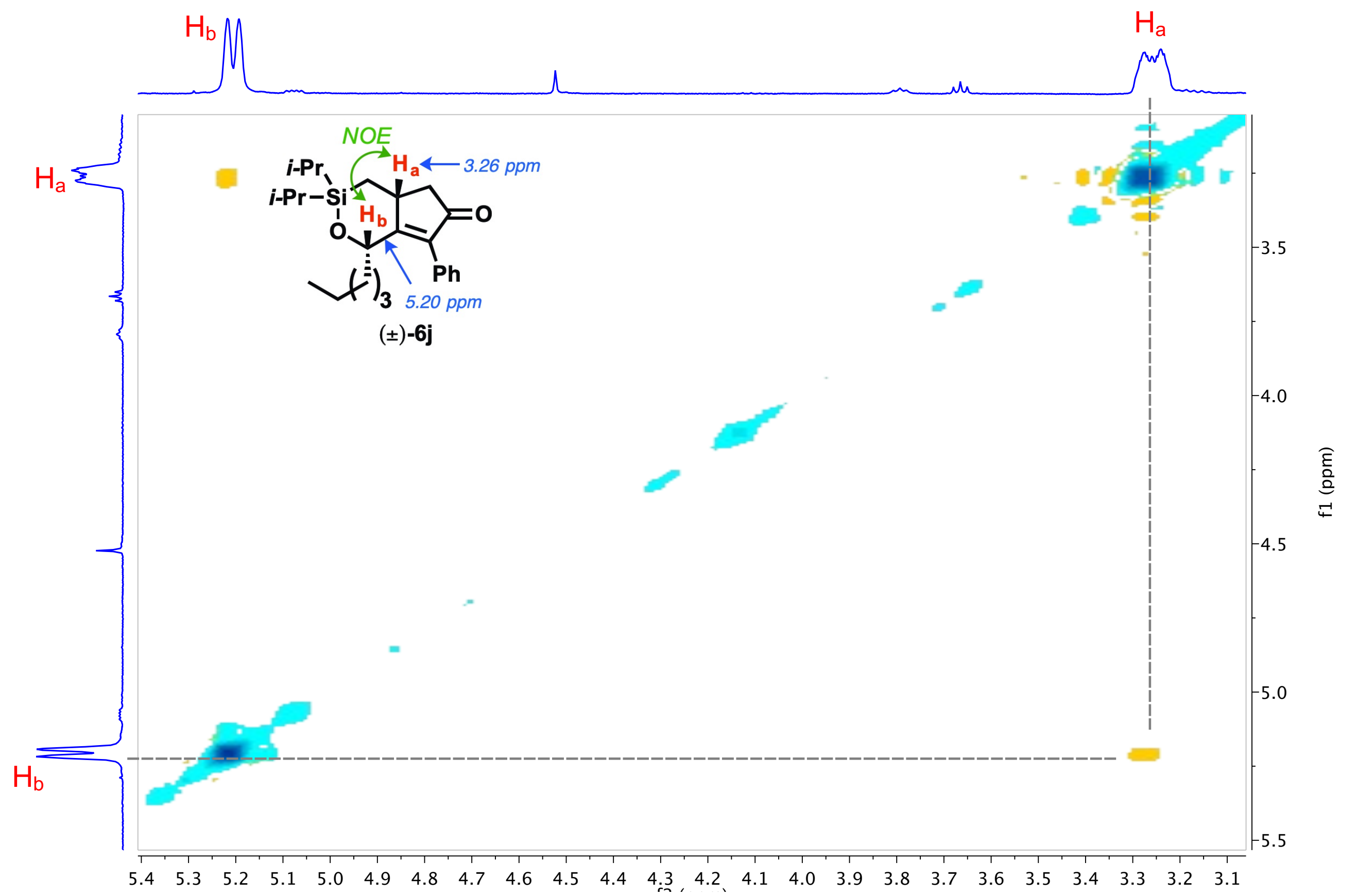




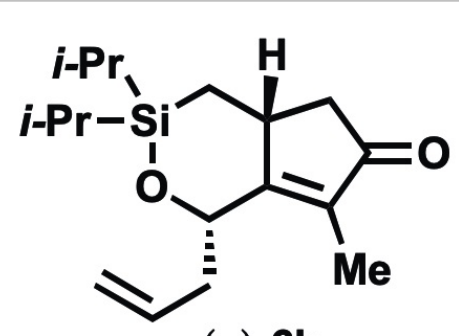

$( \pm)-6 k$

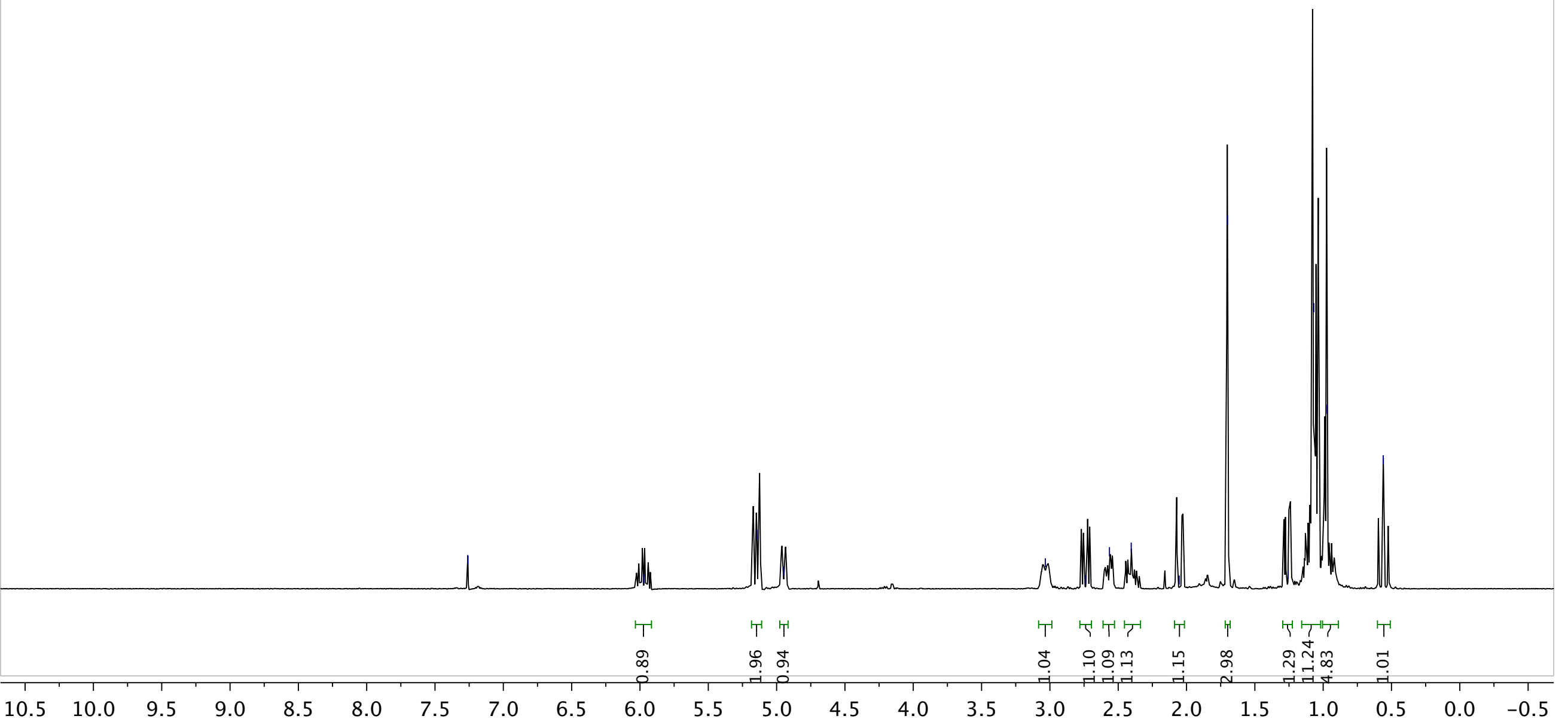




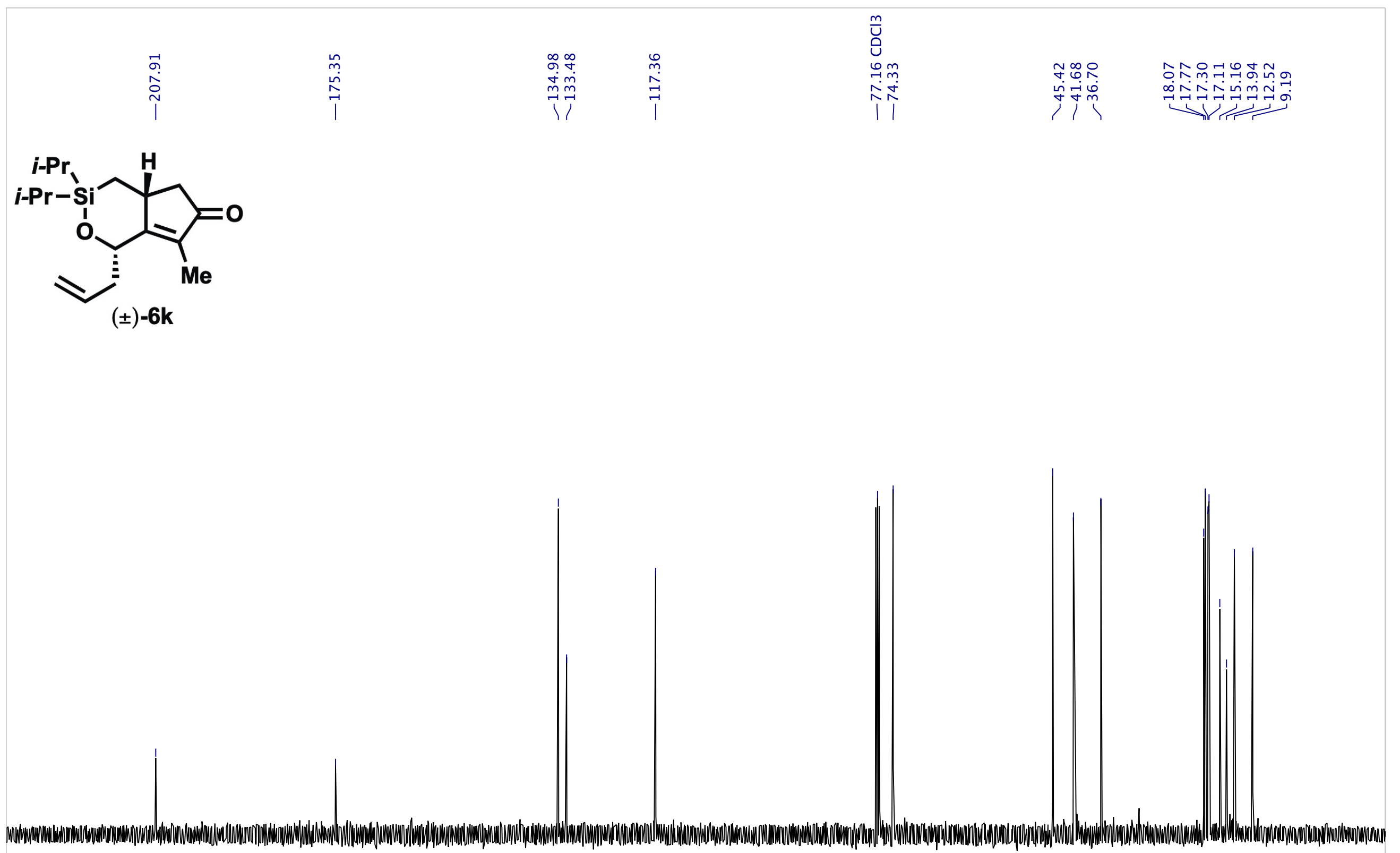

$\begin{array}{lllllllllllllllllllllllll}230 & 220 & 210 & 200 & 190 & 180 & 170 & 160 & 150 & 140 & 130 & 120 & 110 & 100 & 90 & 80 & 70 & 60 & 50 & 40 & 30 & 20 & 10 & 0 & -10\end{array}$




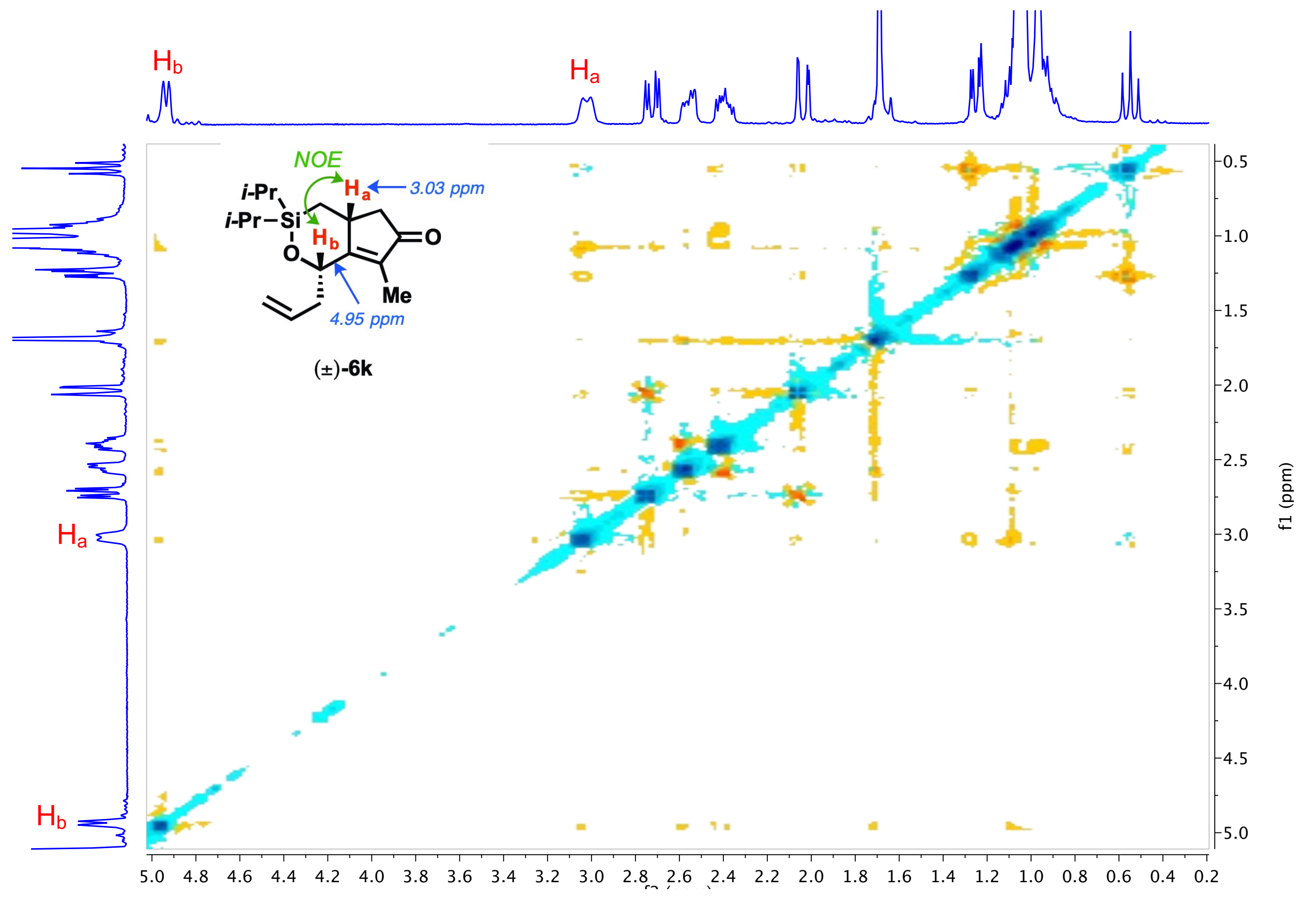




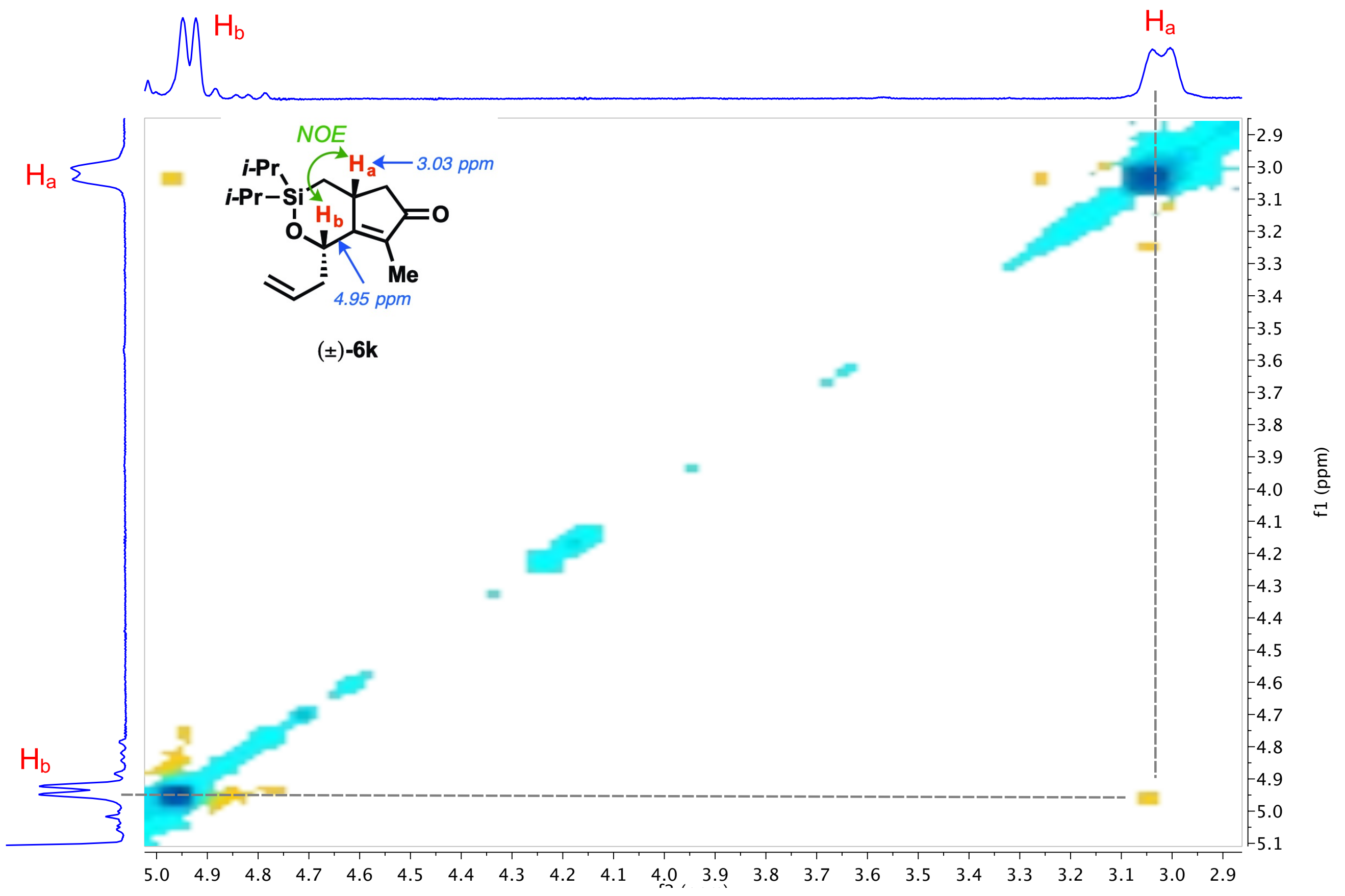



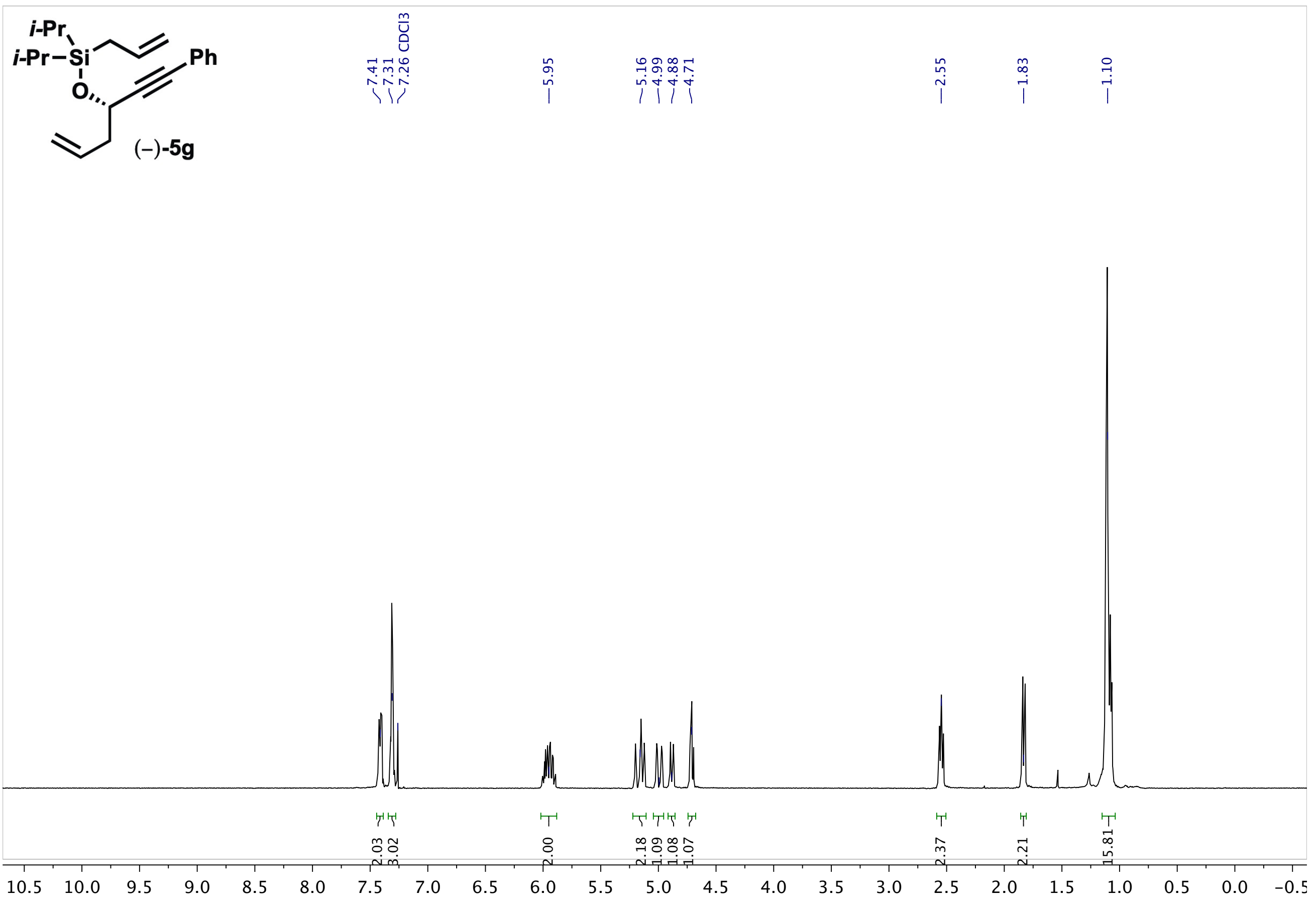

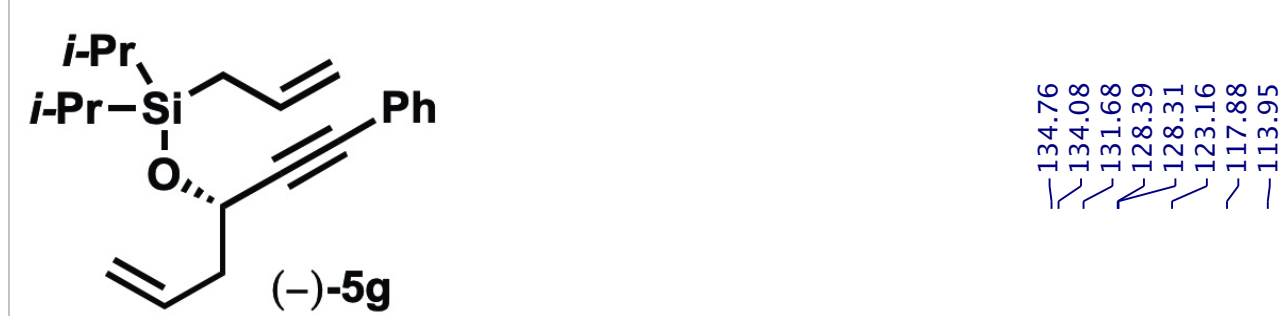

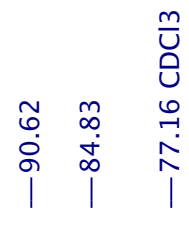

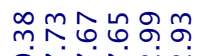
全通弪

$(-)-5 g$

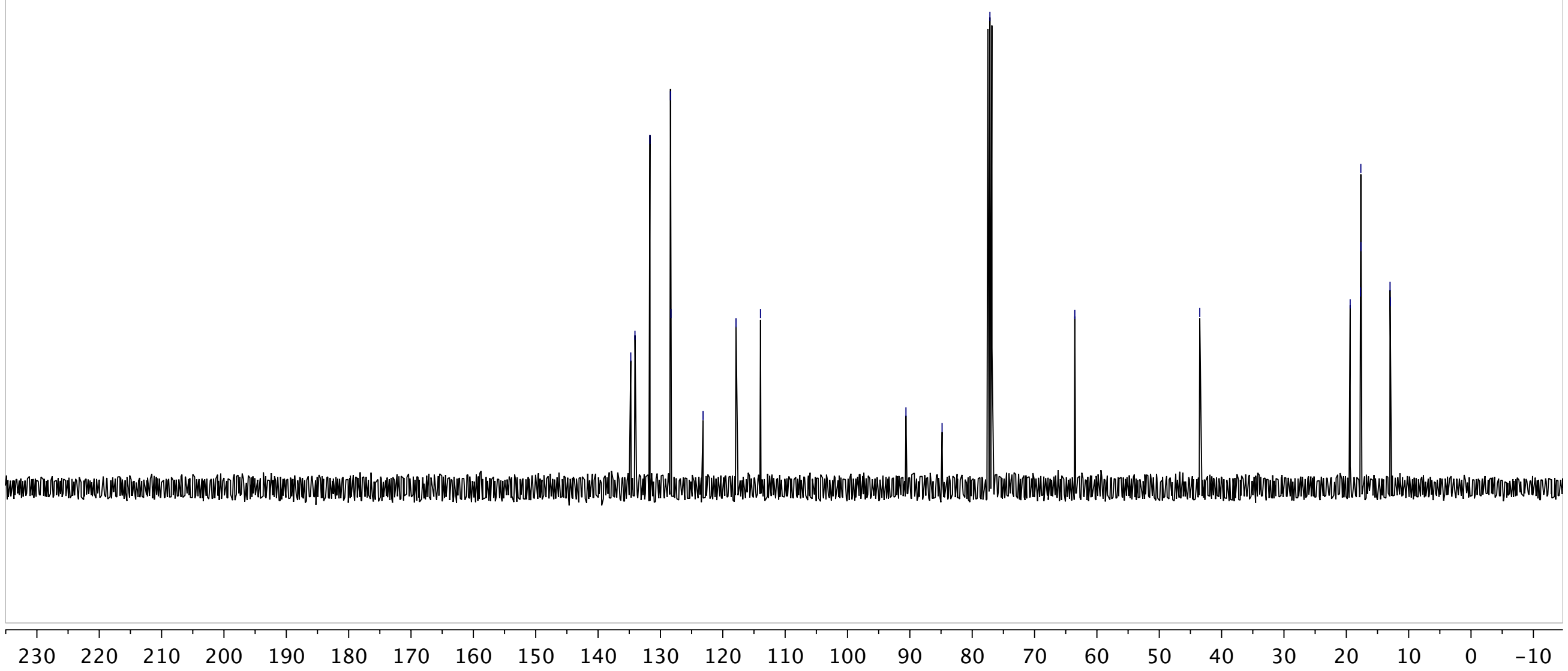



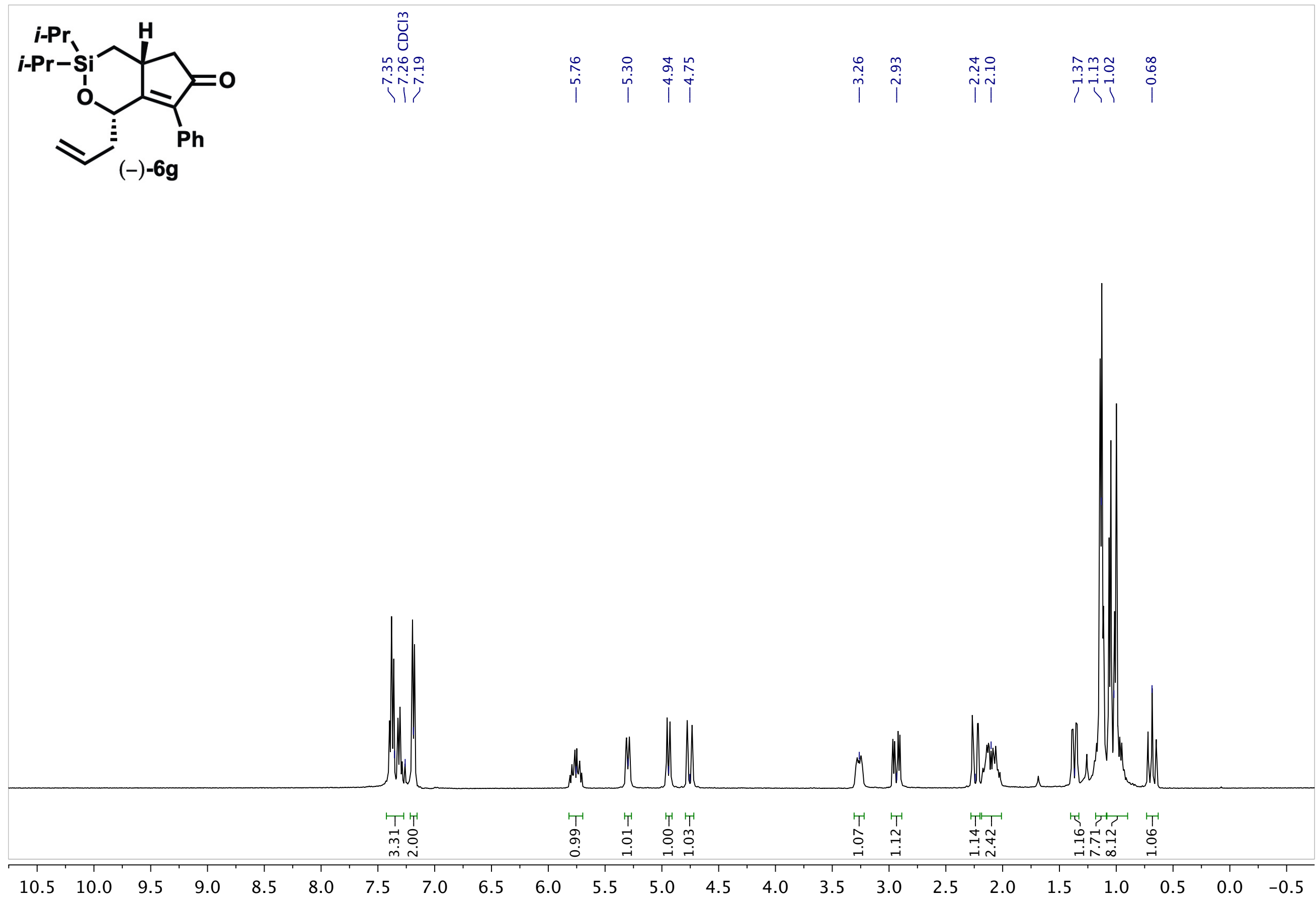


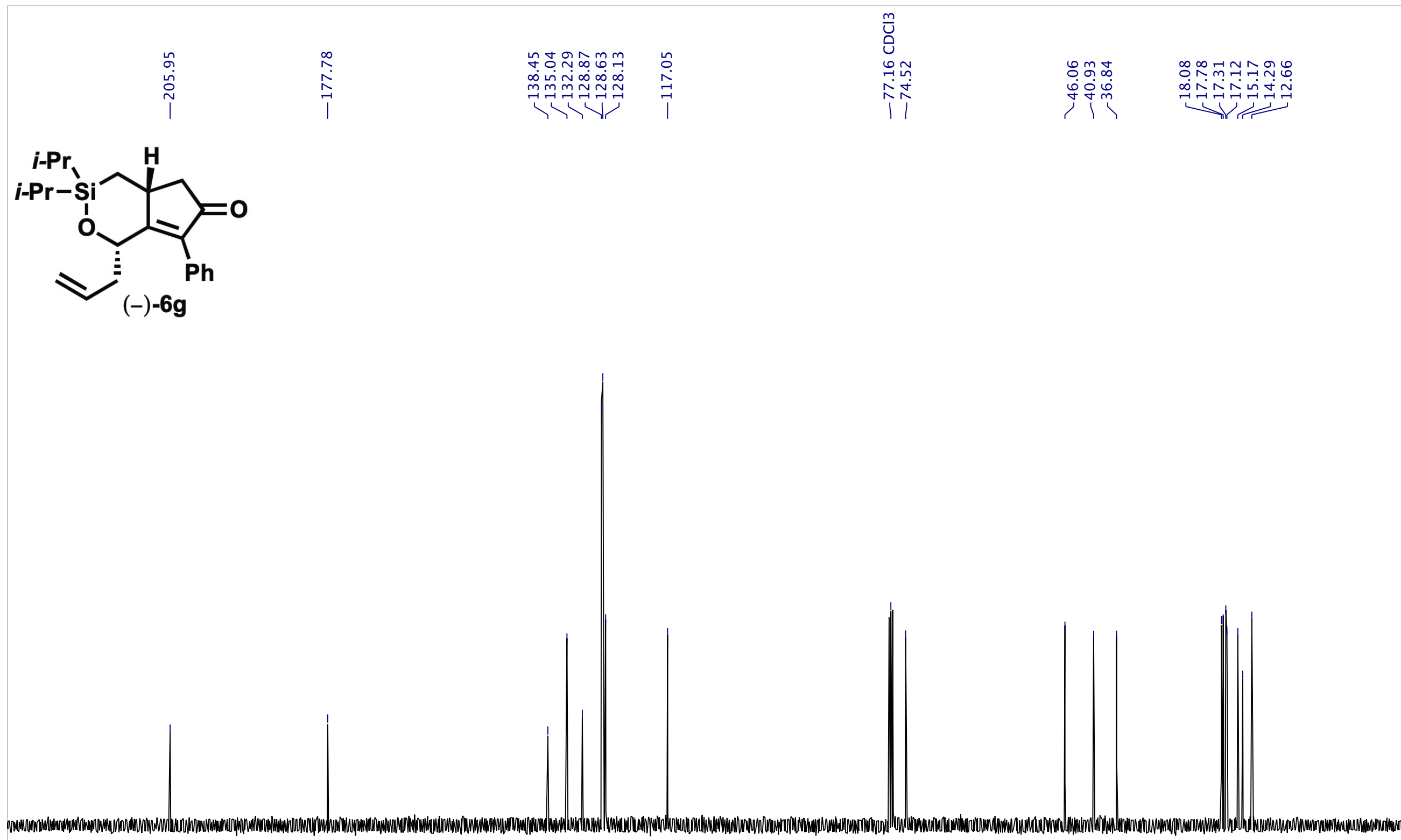

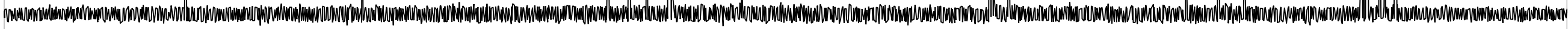

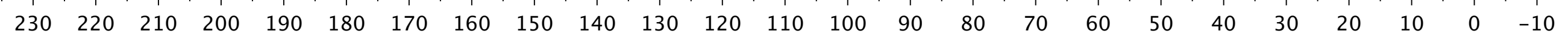




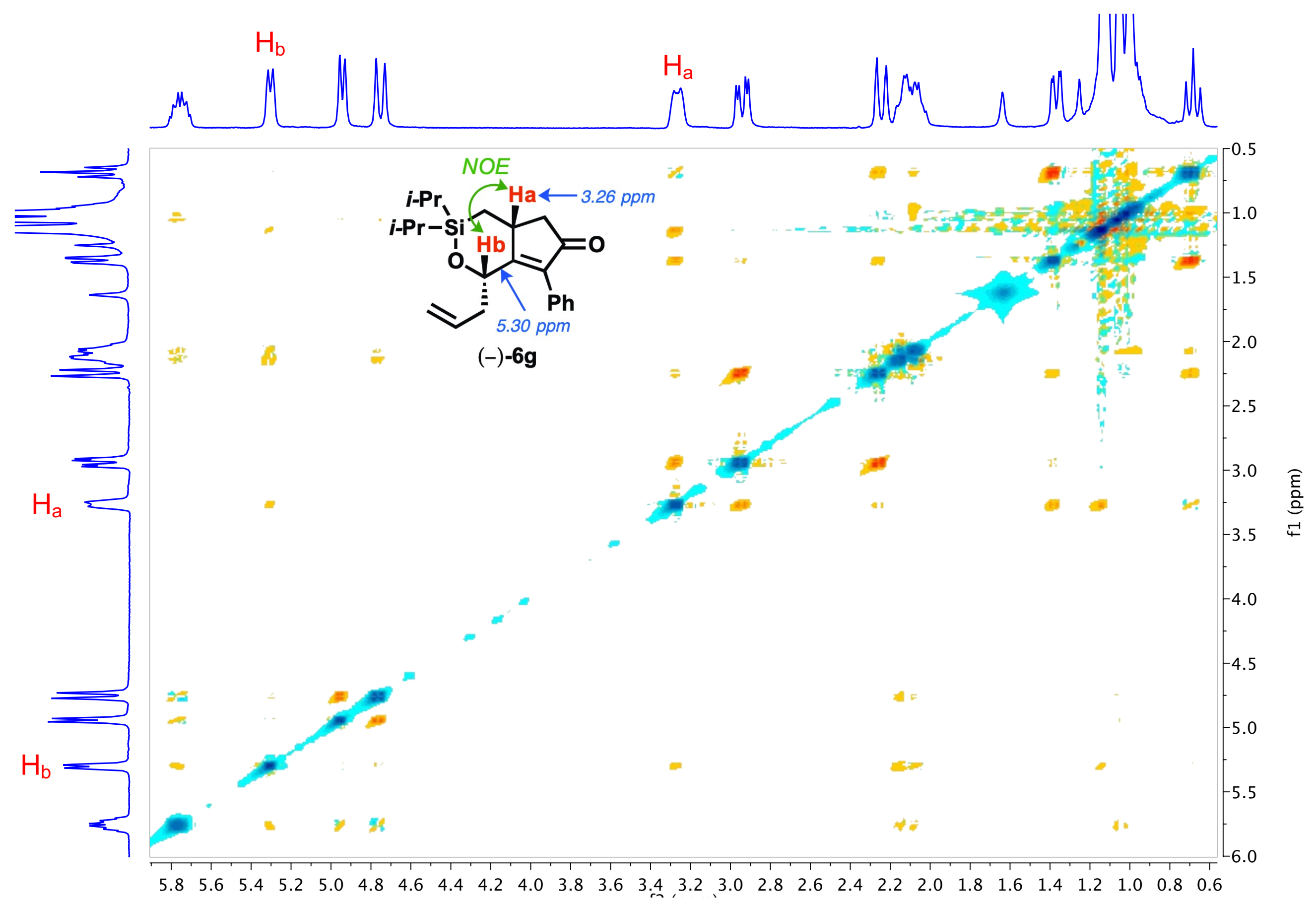




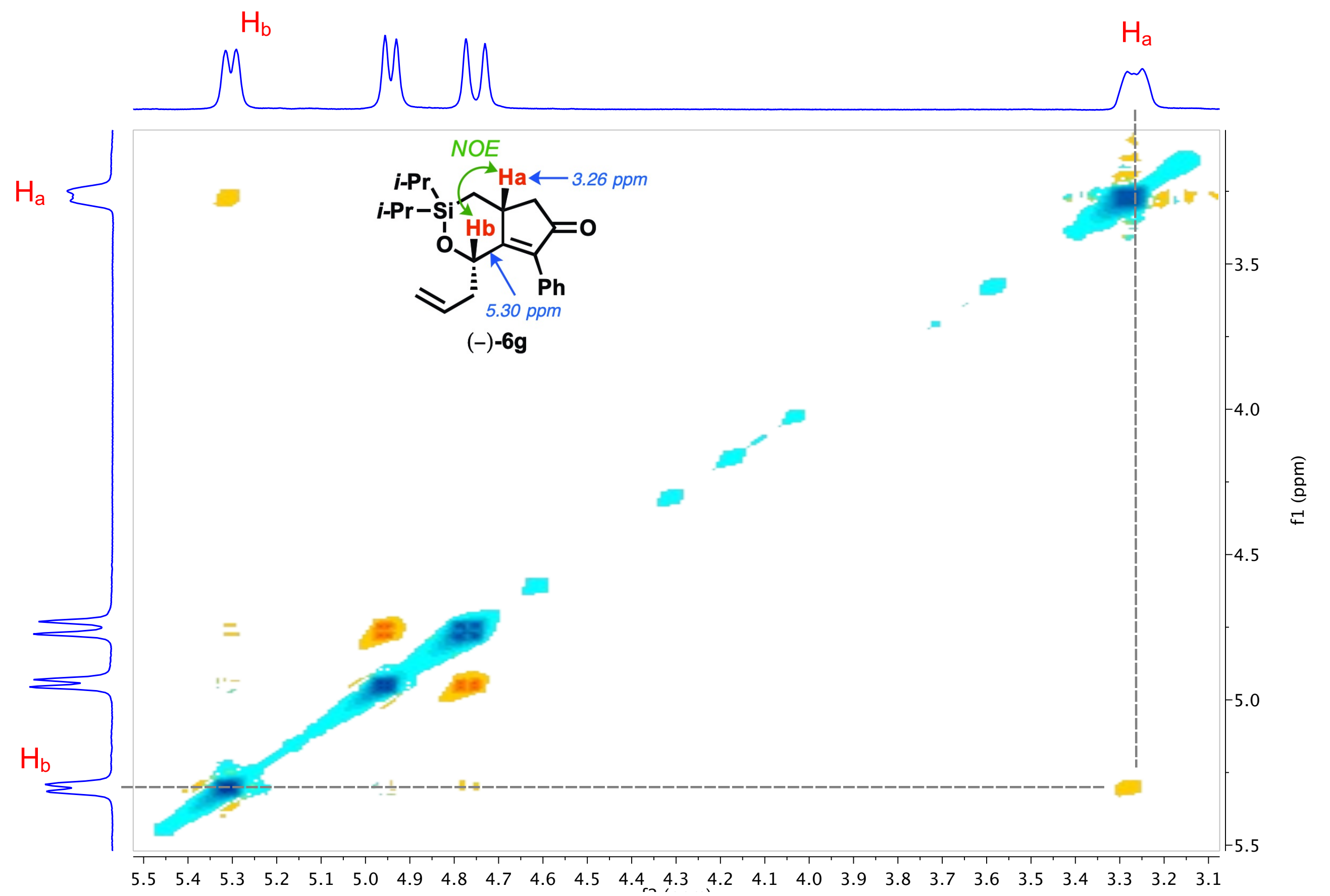



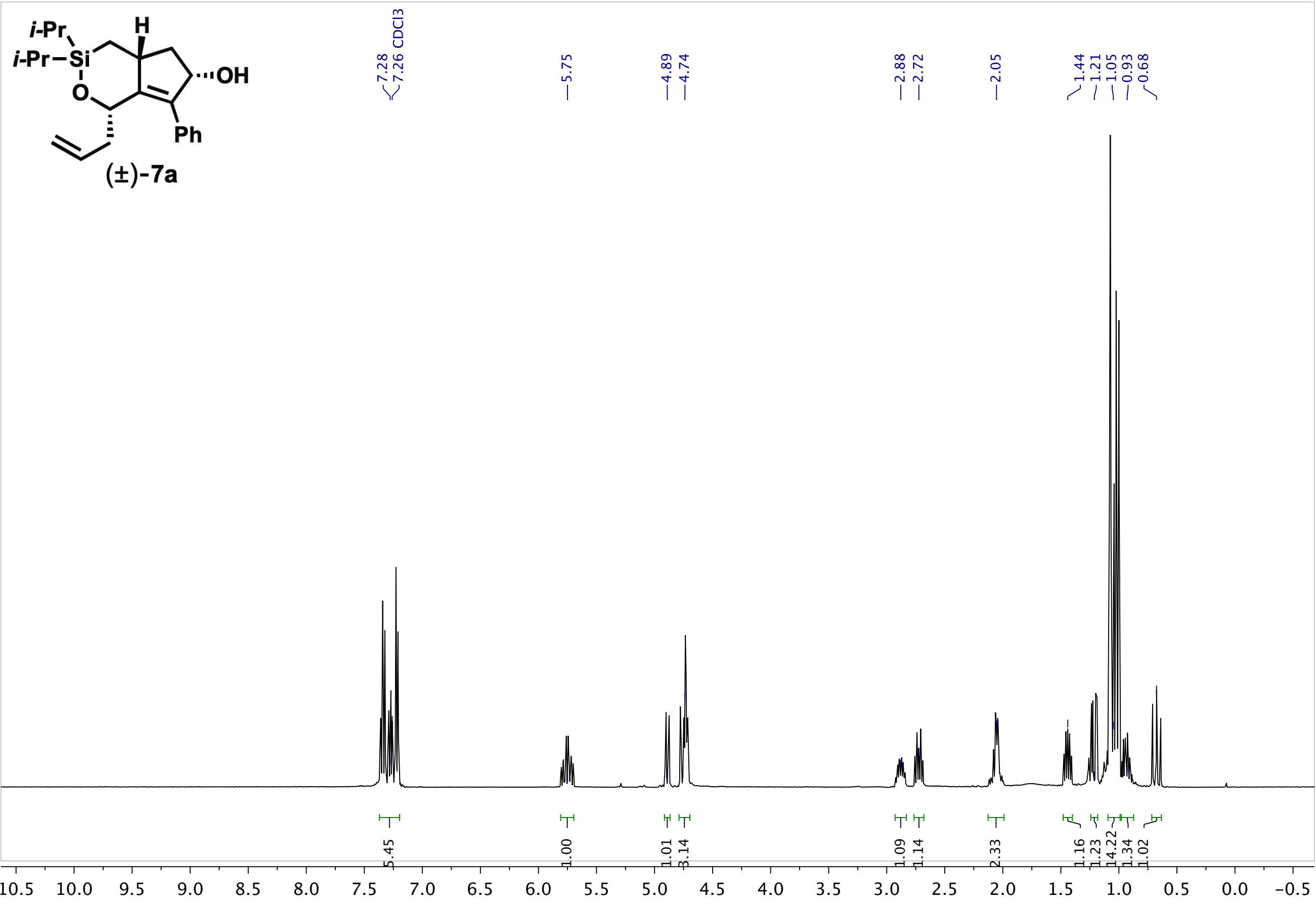
<smiles>C=CC[C@H]1O[Si](CC)(C([In])[In])C[C@H]2C[C@H](O)C(c3ccccc3)=C21</smiles>

$( \pm)-7 a$
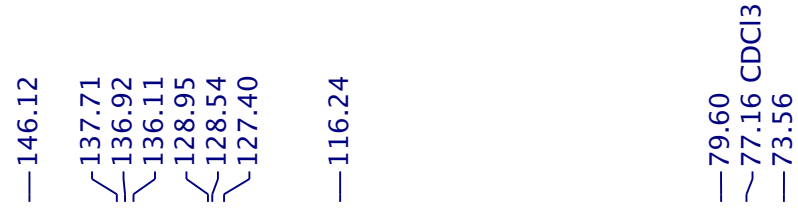

이요

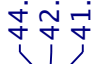

$\checkmark \infty m \infty$ m

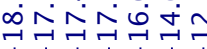

保

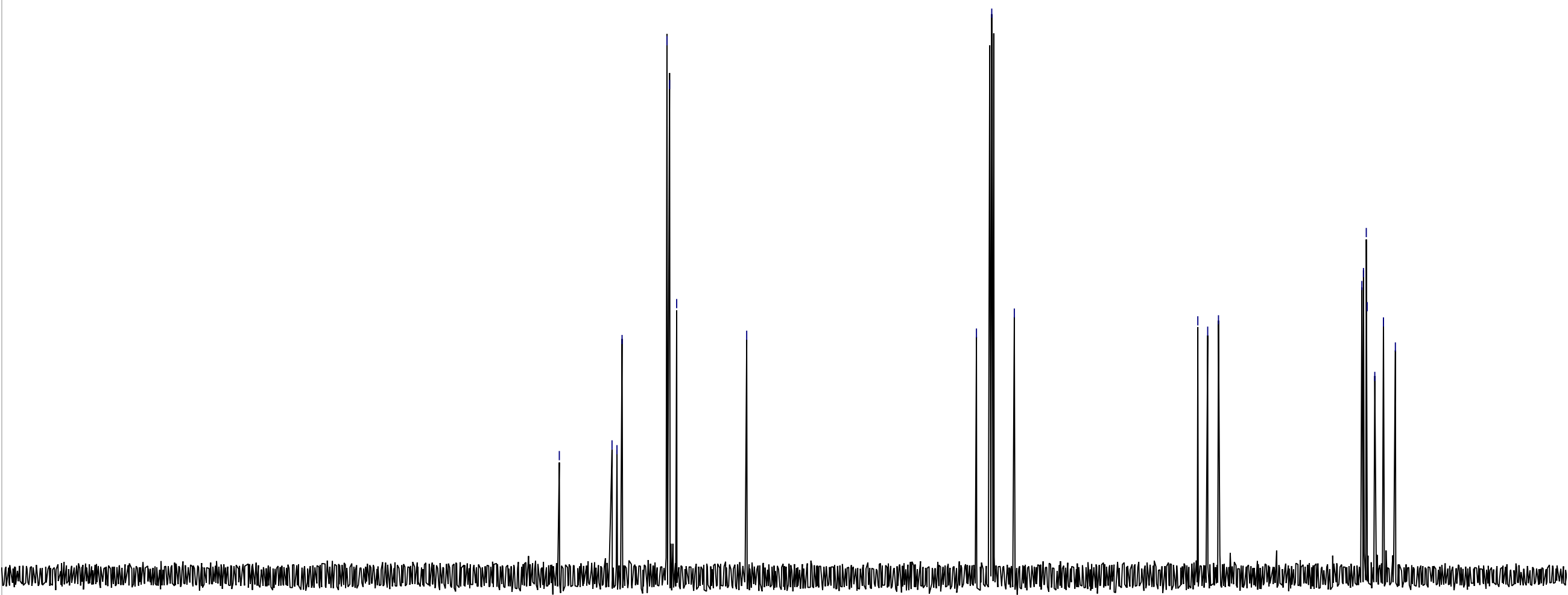

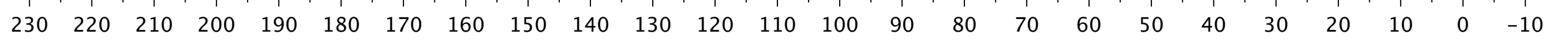




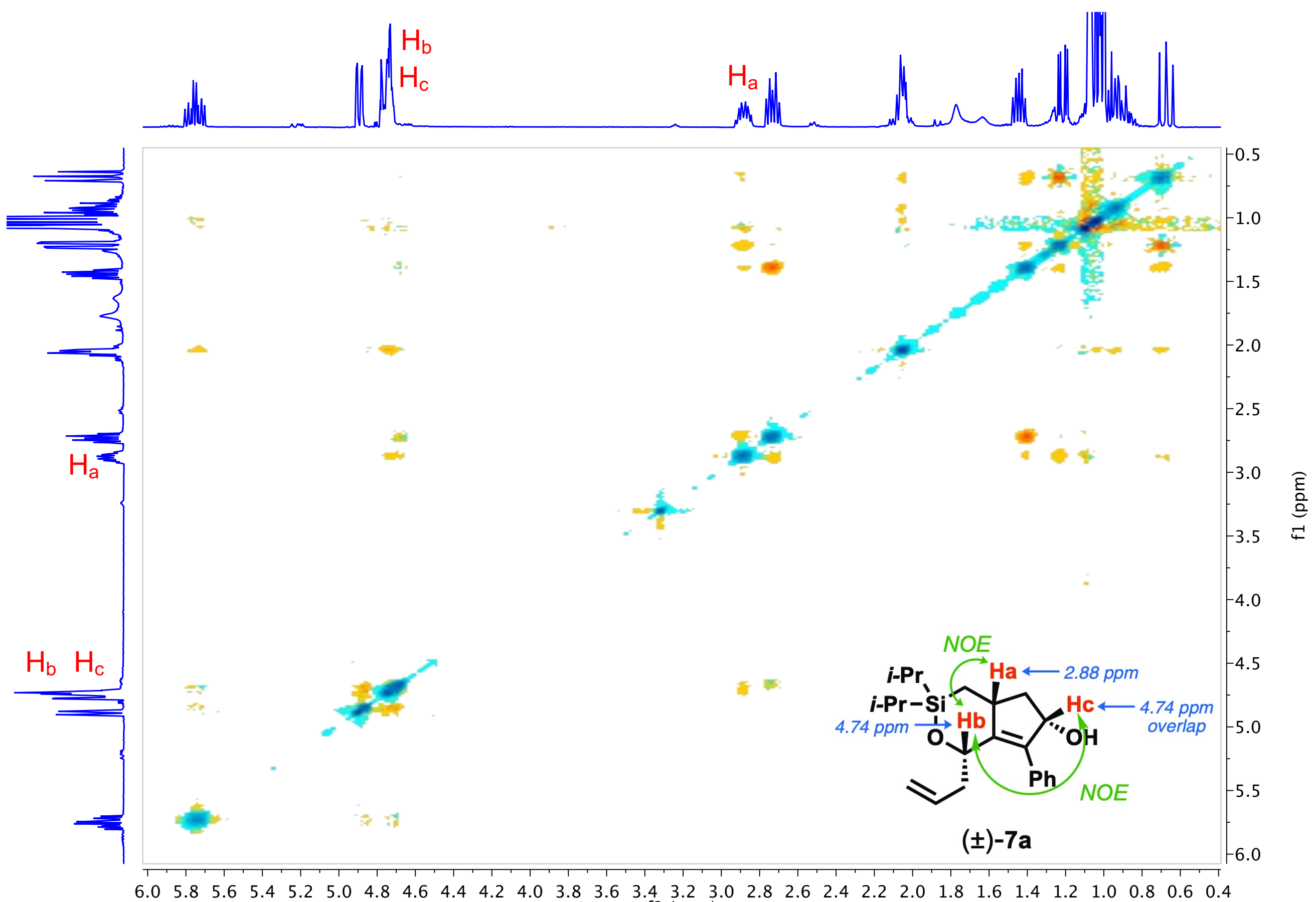




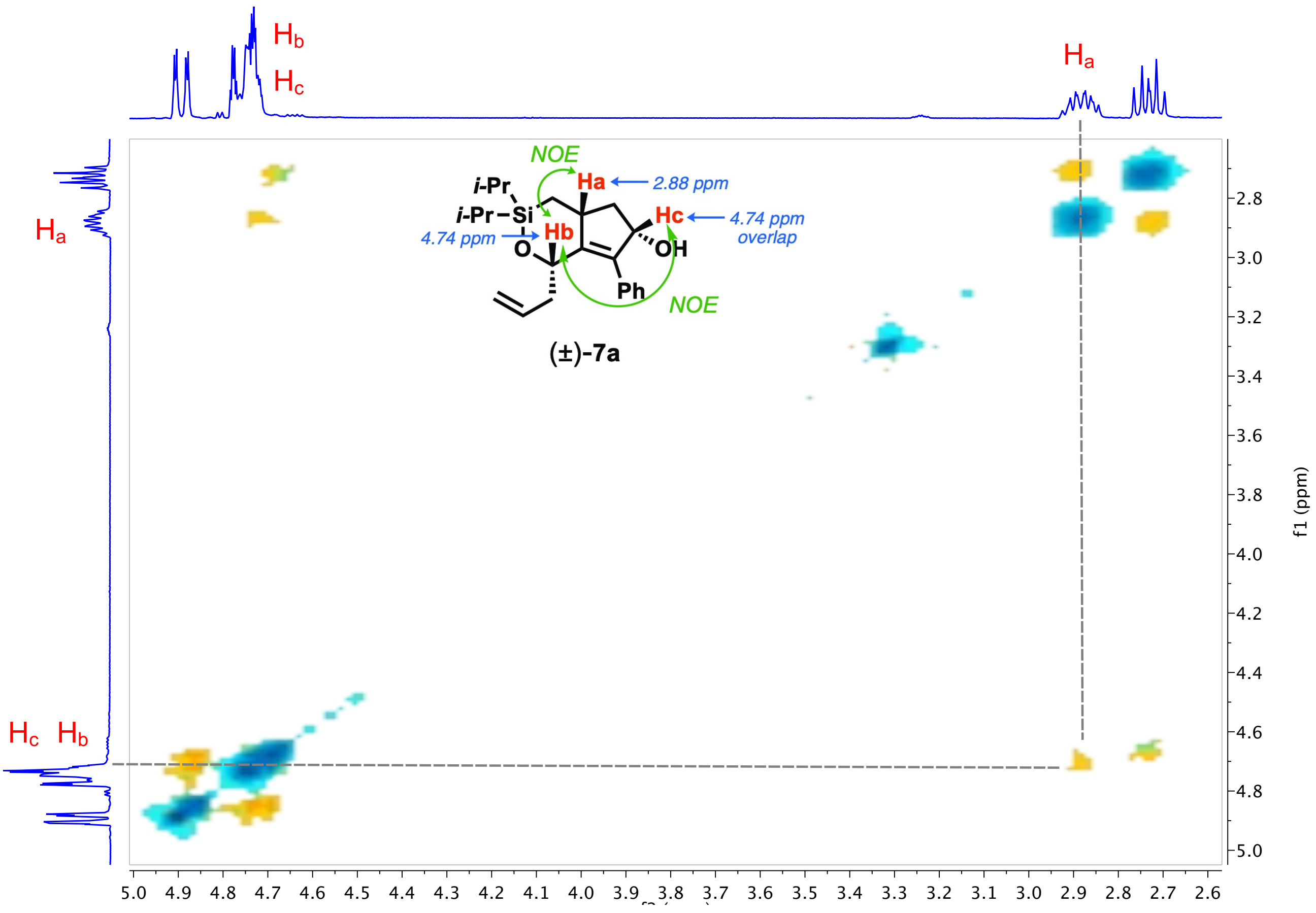



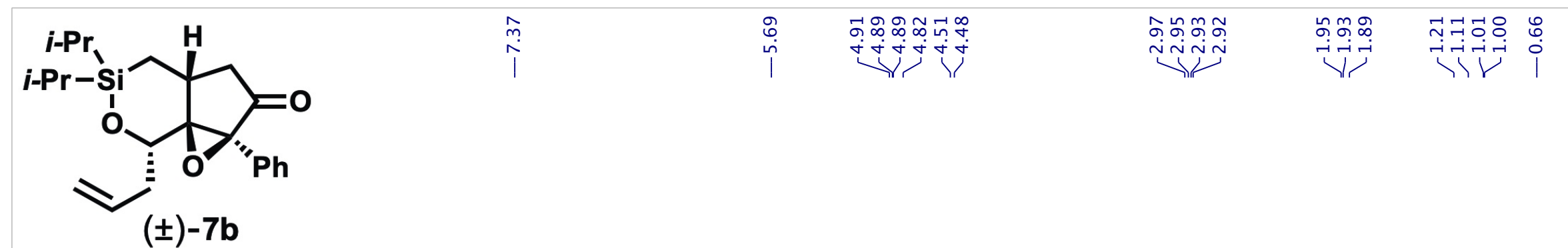

ลิดูกูดู สู

กำ

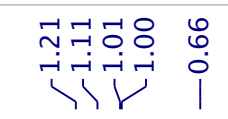

(士)-7b

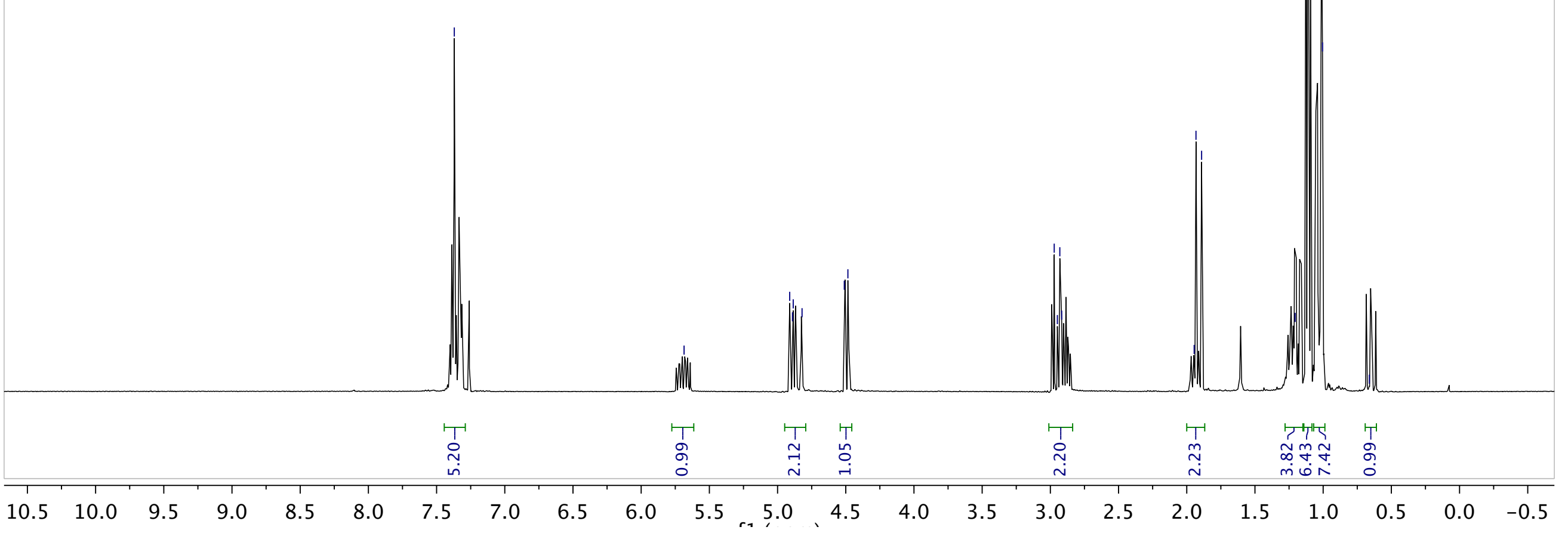




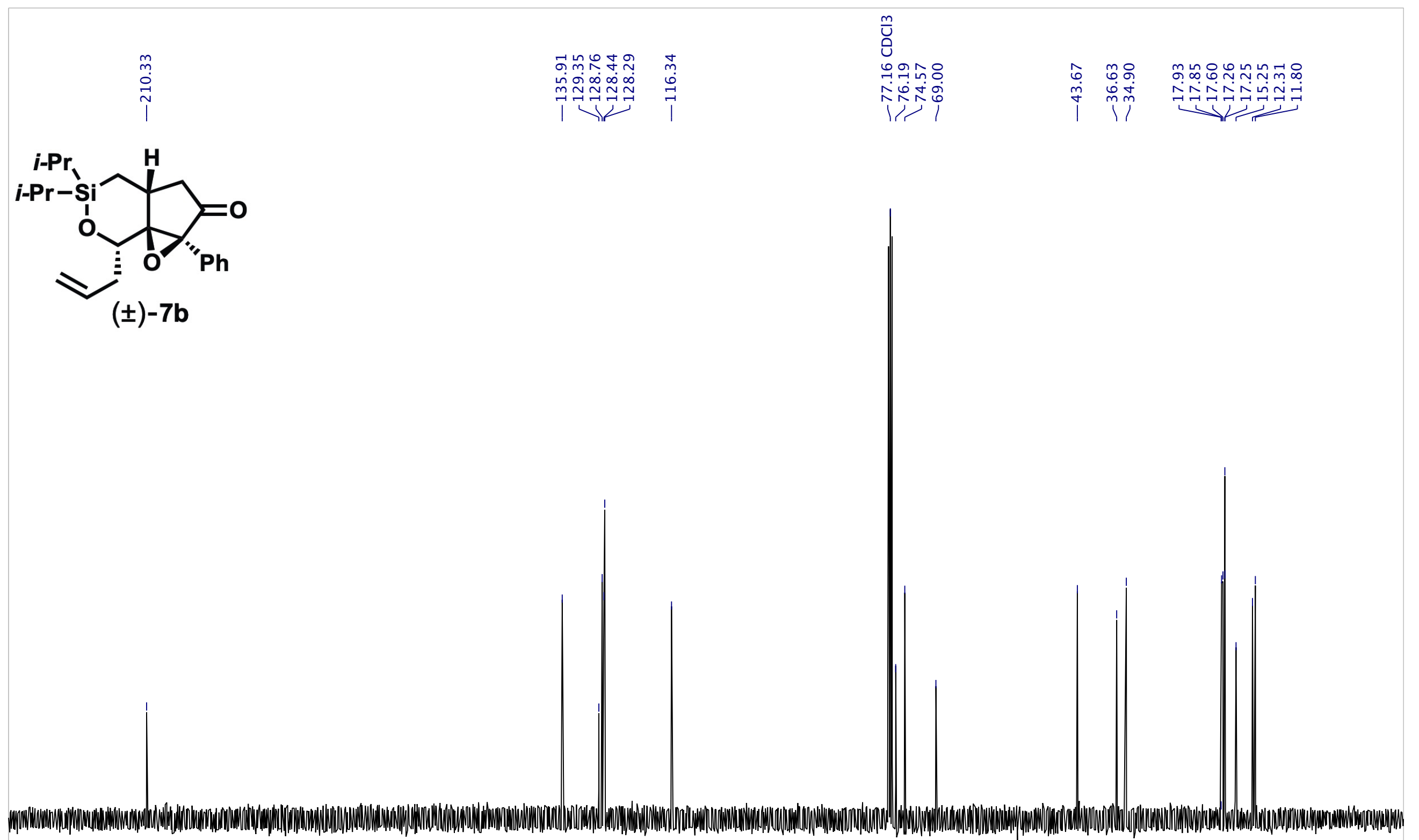

$\begin{array}{lllllllllllllllllllllllll}230 & 220 & 210 & 200 & 190 & 180 & 170 & 160 & 150 & 140 & 130 & 120 & 110 & 100 & 90 & 80 & 70 & 60 & 50 & 40 & 30 & 20 & 10 & 0 & -10\end{array}$




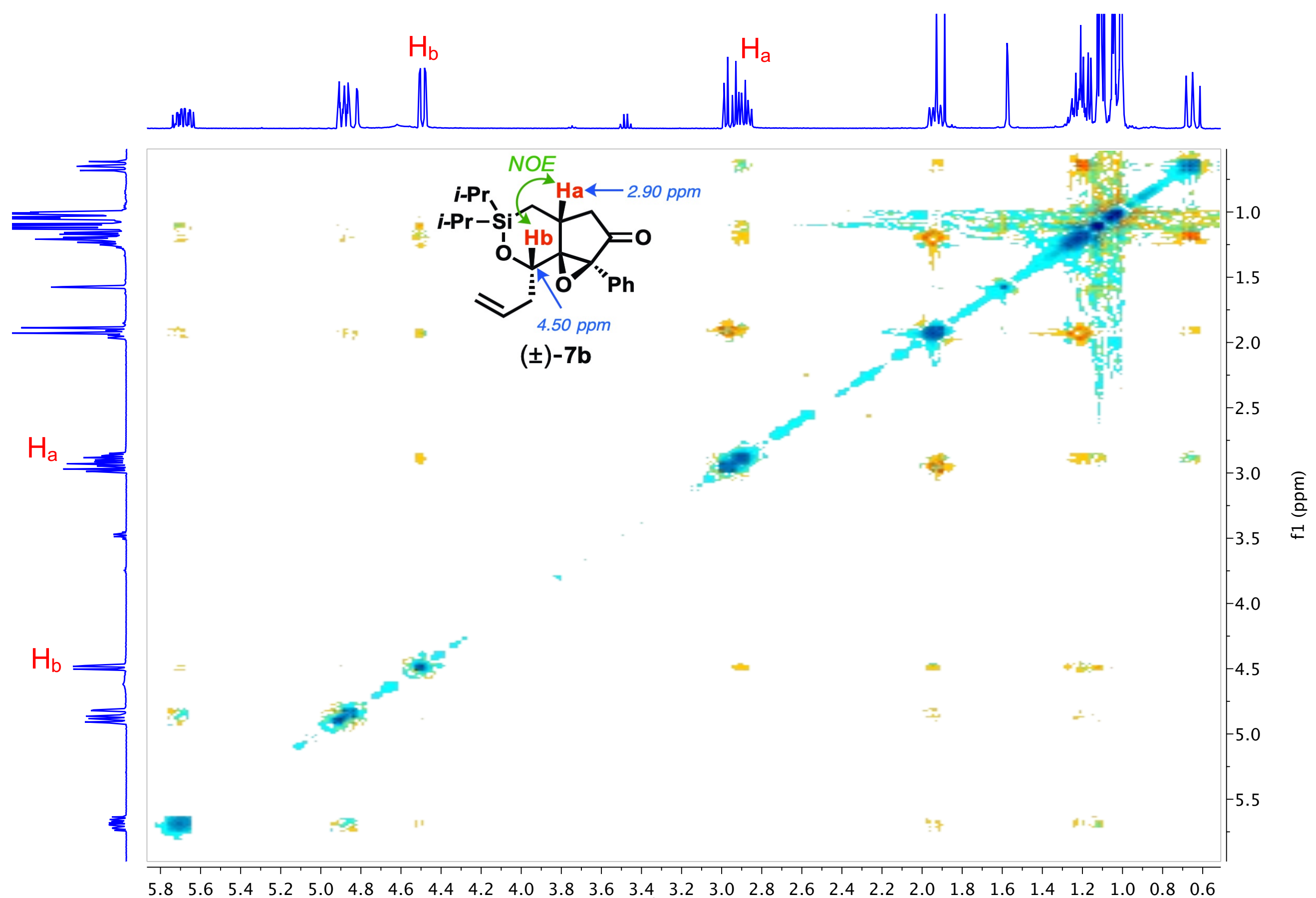




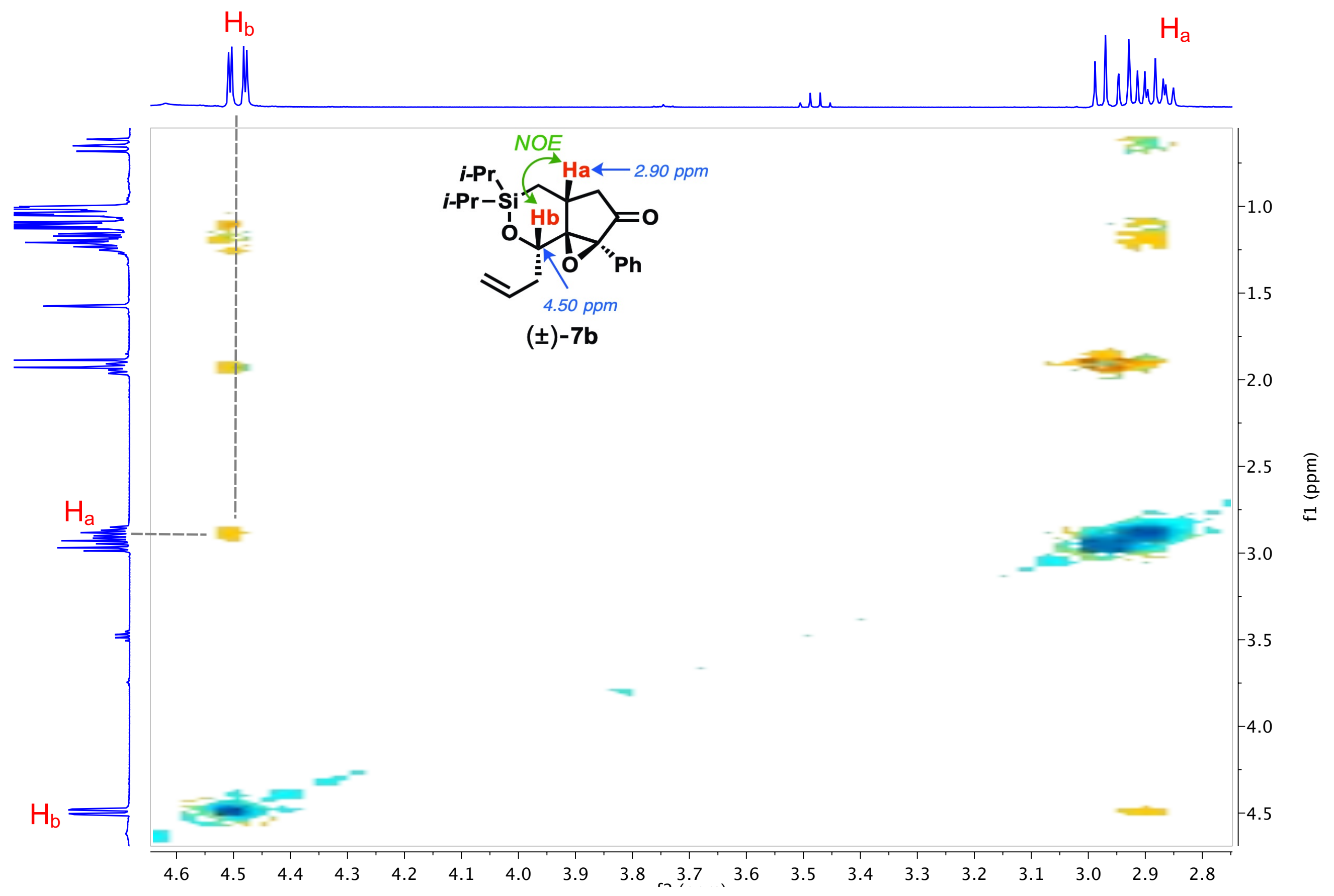




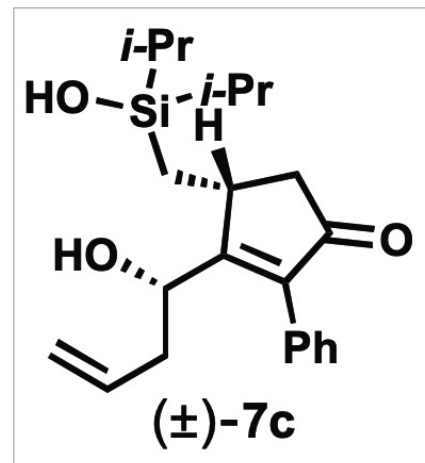

$( \pm)-7 c$

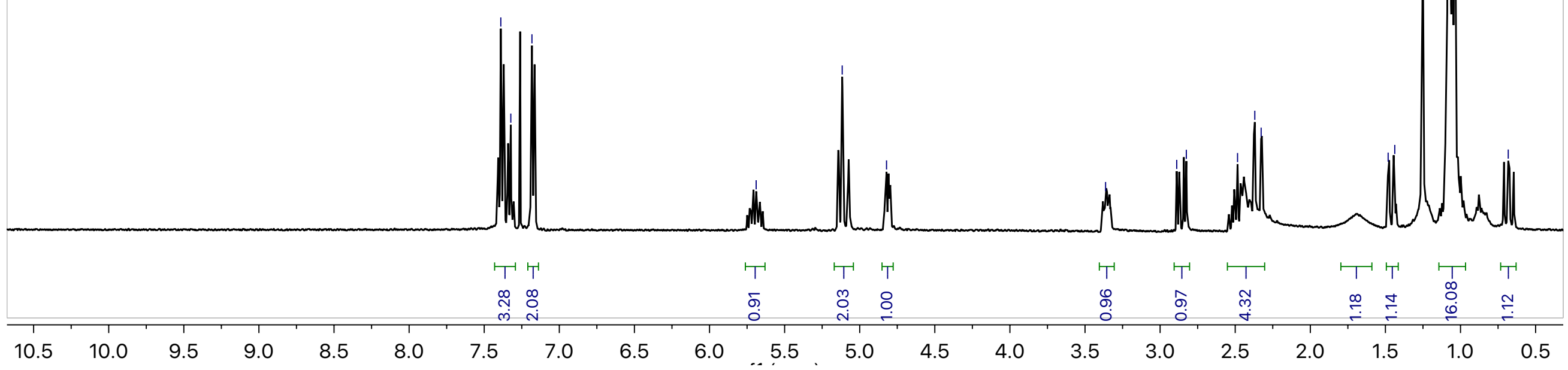




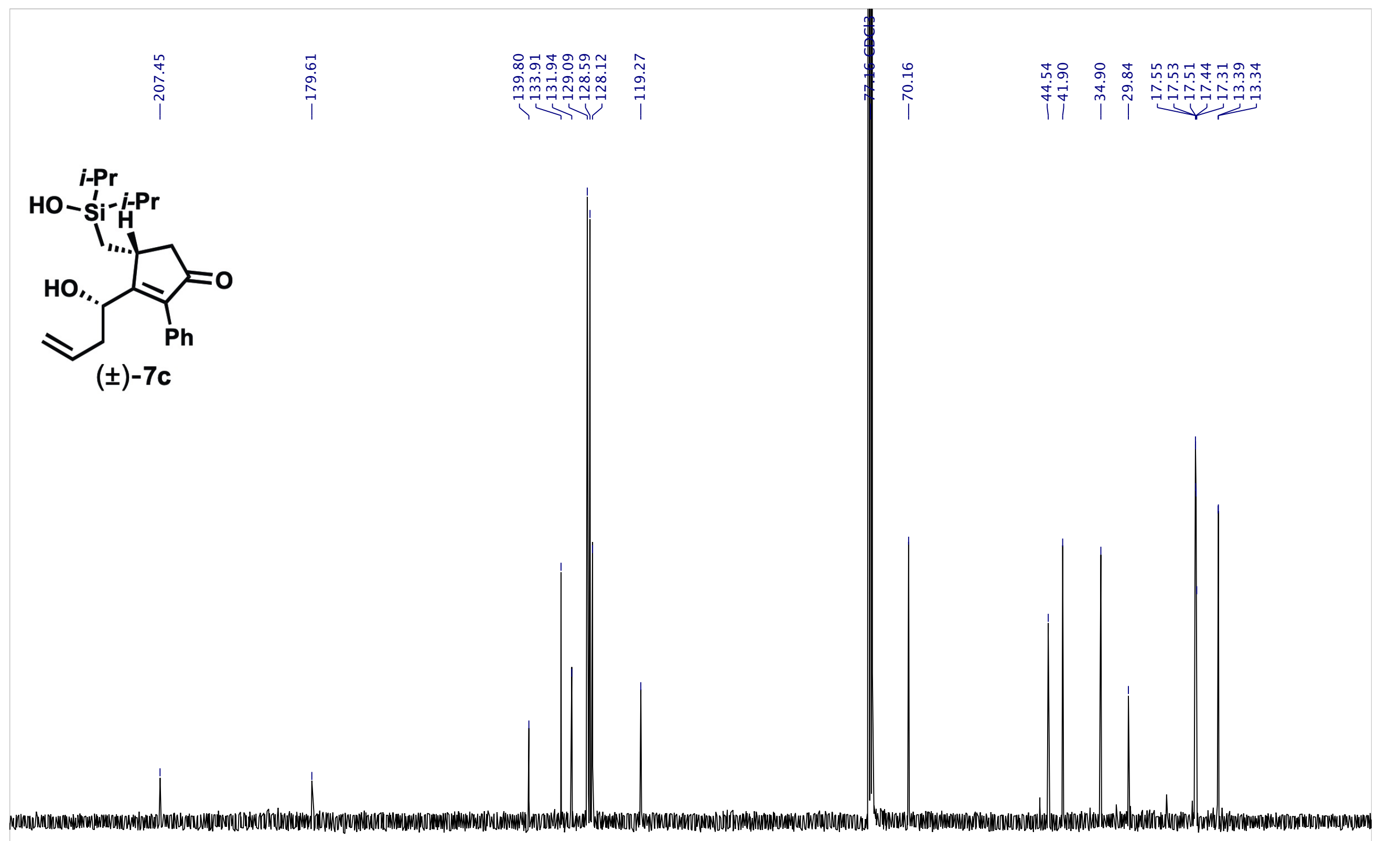

$\begin{array}{llllllllllllllllllllllllllllll}230 & 220 & 210 & 200 & 190 & 180 & 170 & 160 & 150 & 140 & 130 & 120 & 110 & 100 & 90 & 80 & 70 & 60 & 50 & 40 & 30 & 20 & 10 & 0 & -10\end{array}$




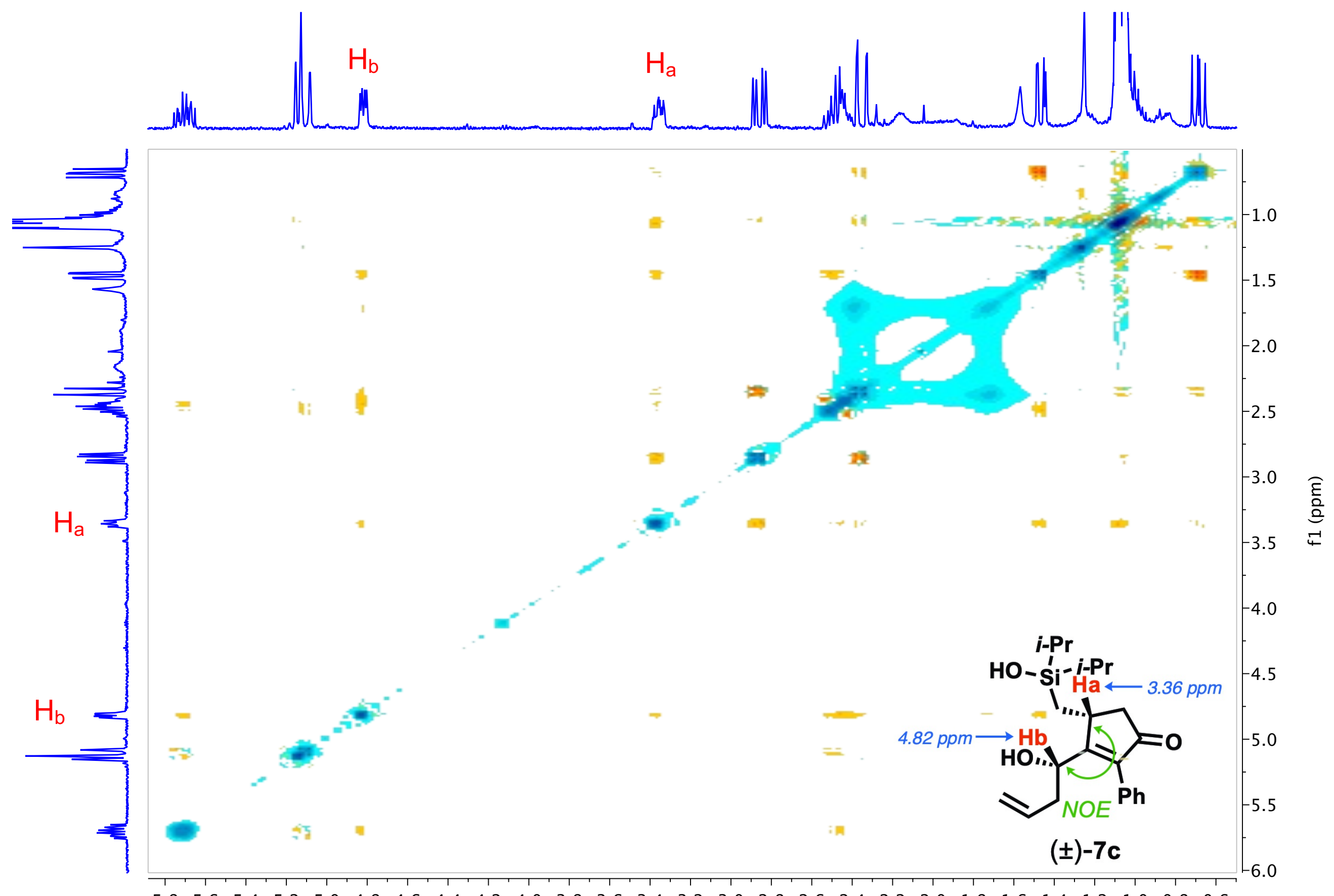




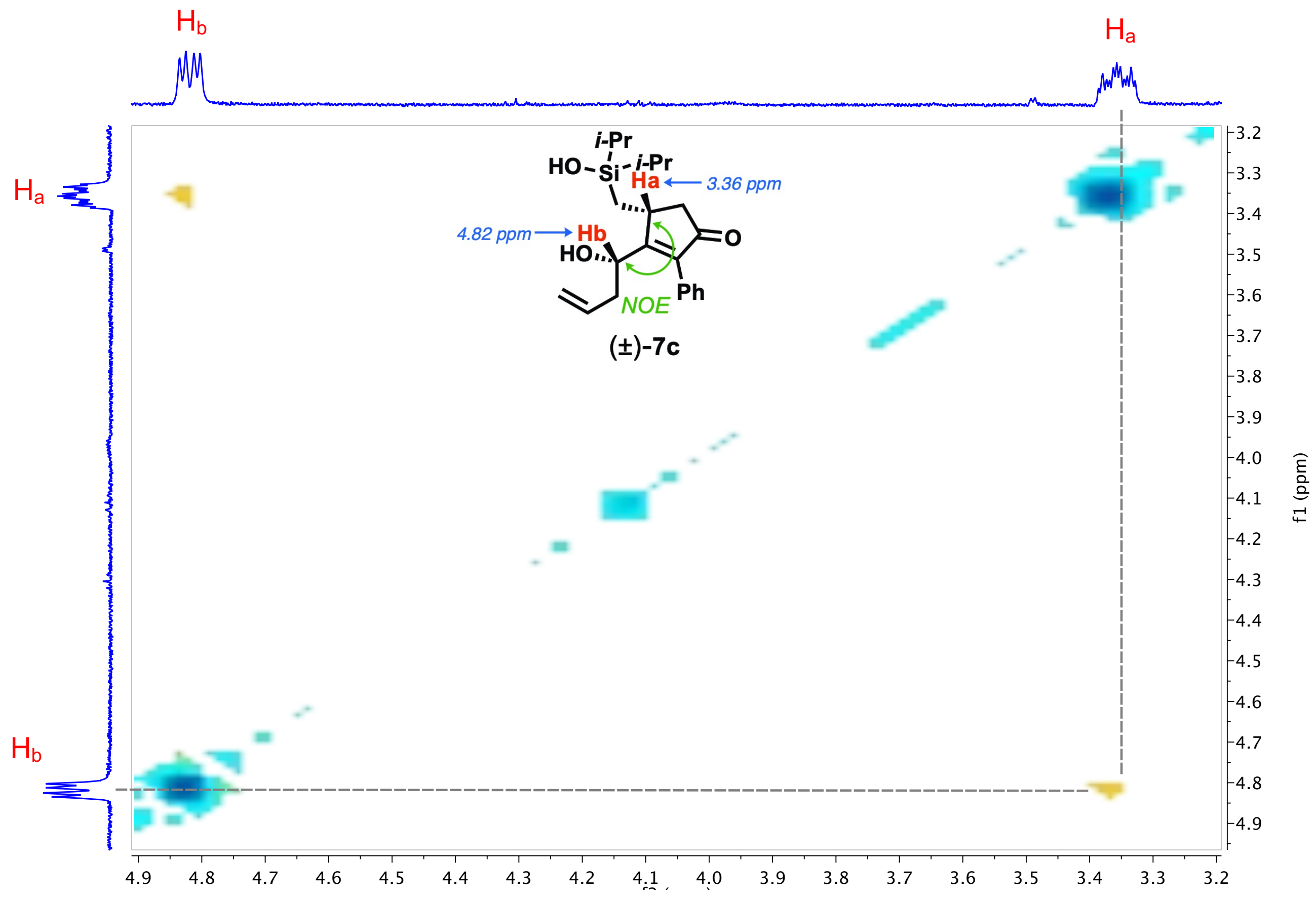



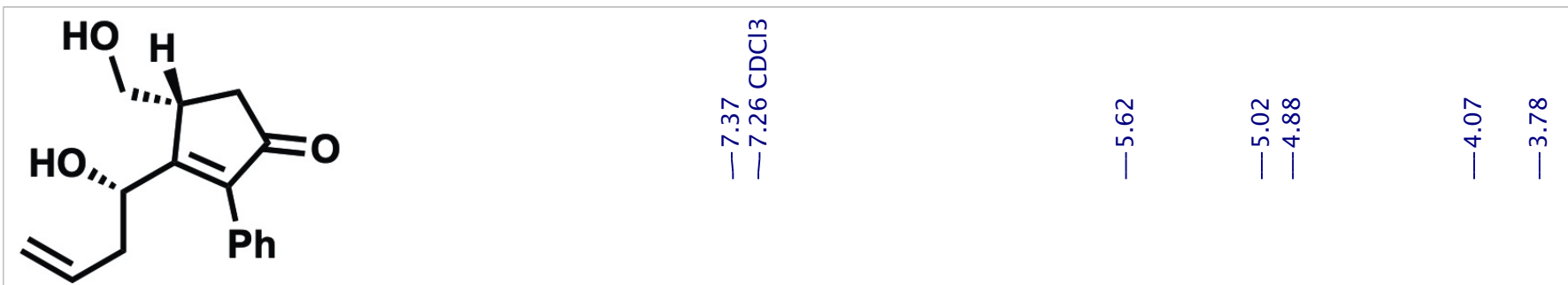

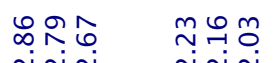

रंग भij

$( \pm)-7 d$

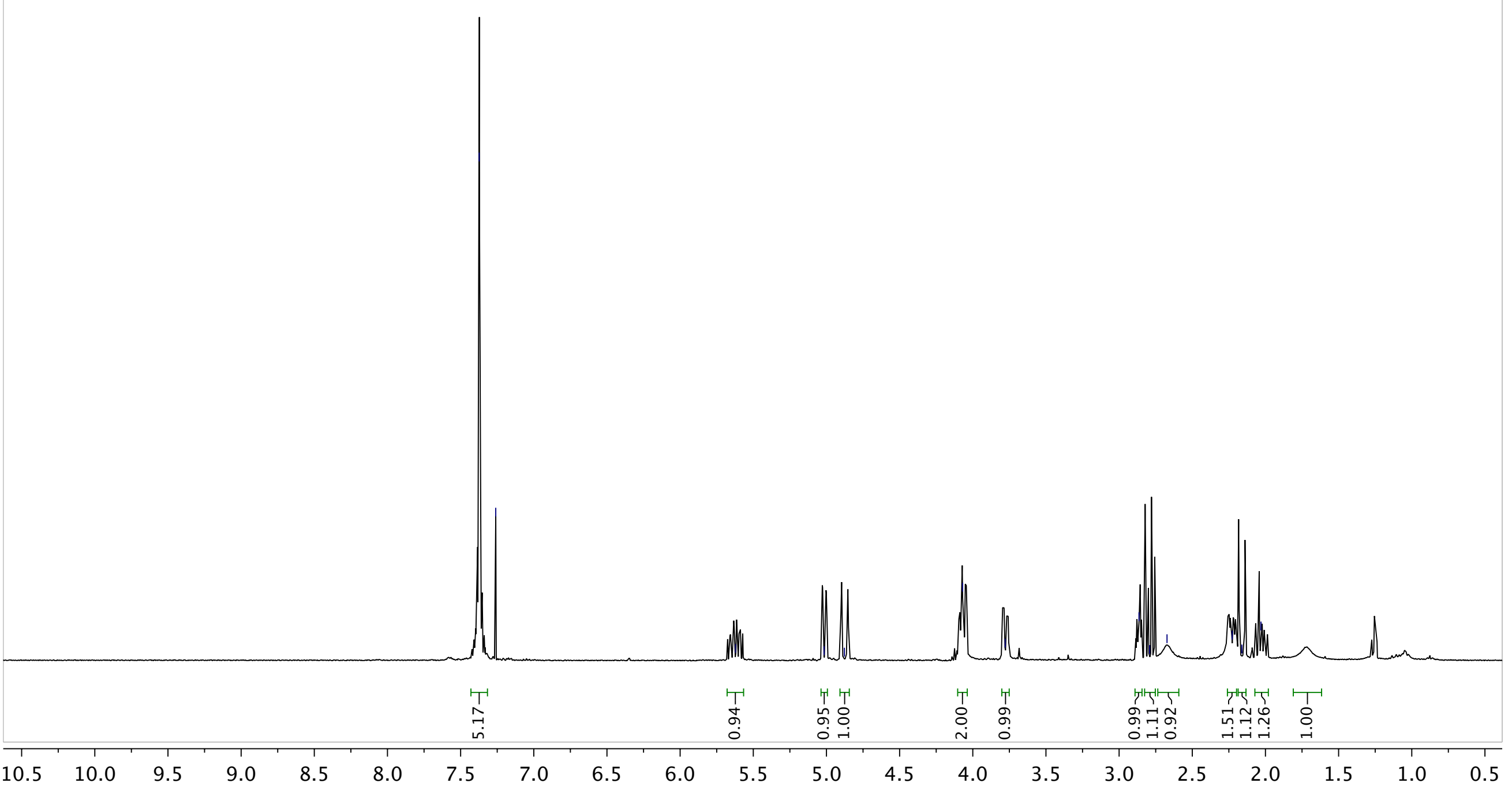




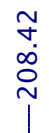

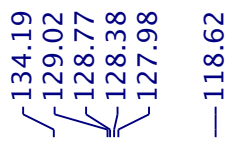

무요

소워ำ

$+11$

ำำำ

minn

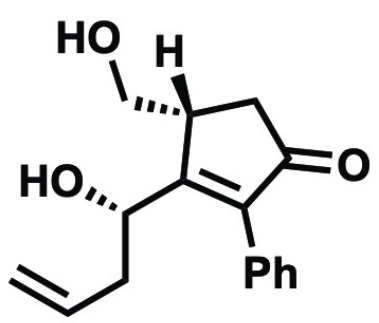

$( \pm)-7 d$
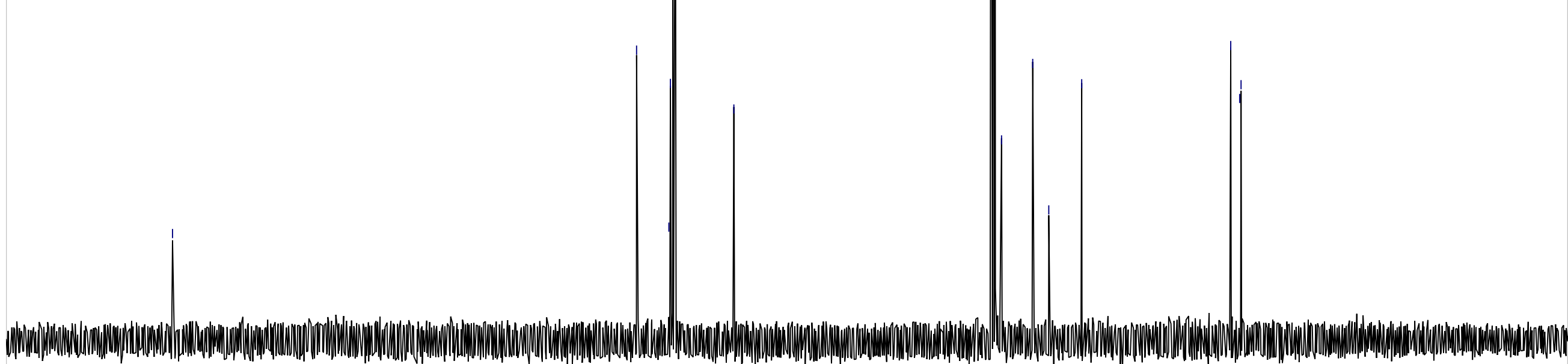


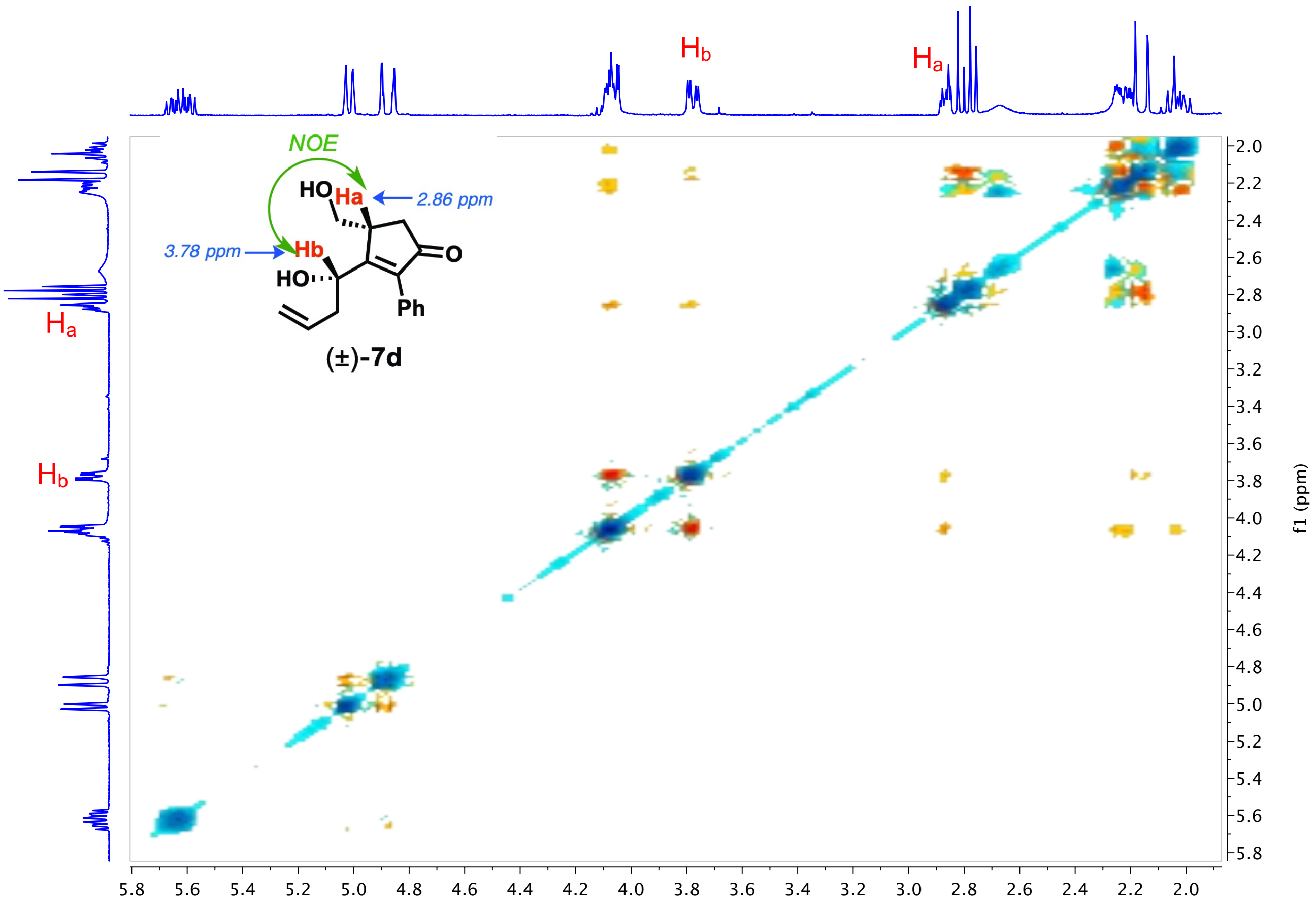




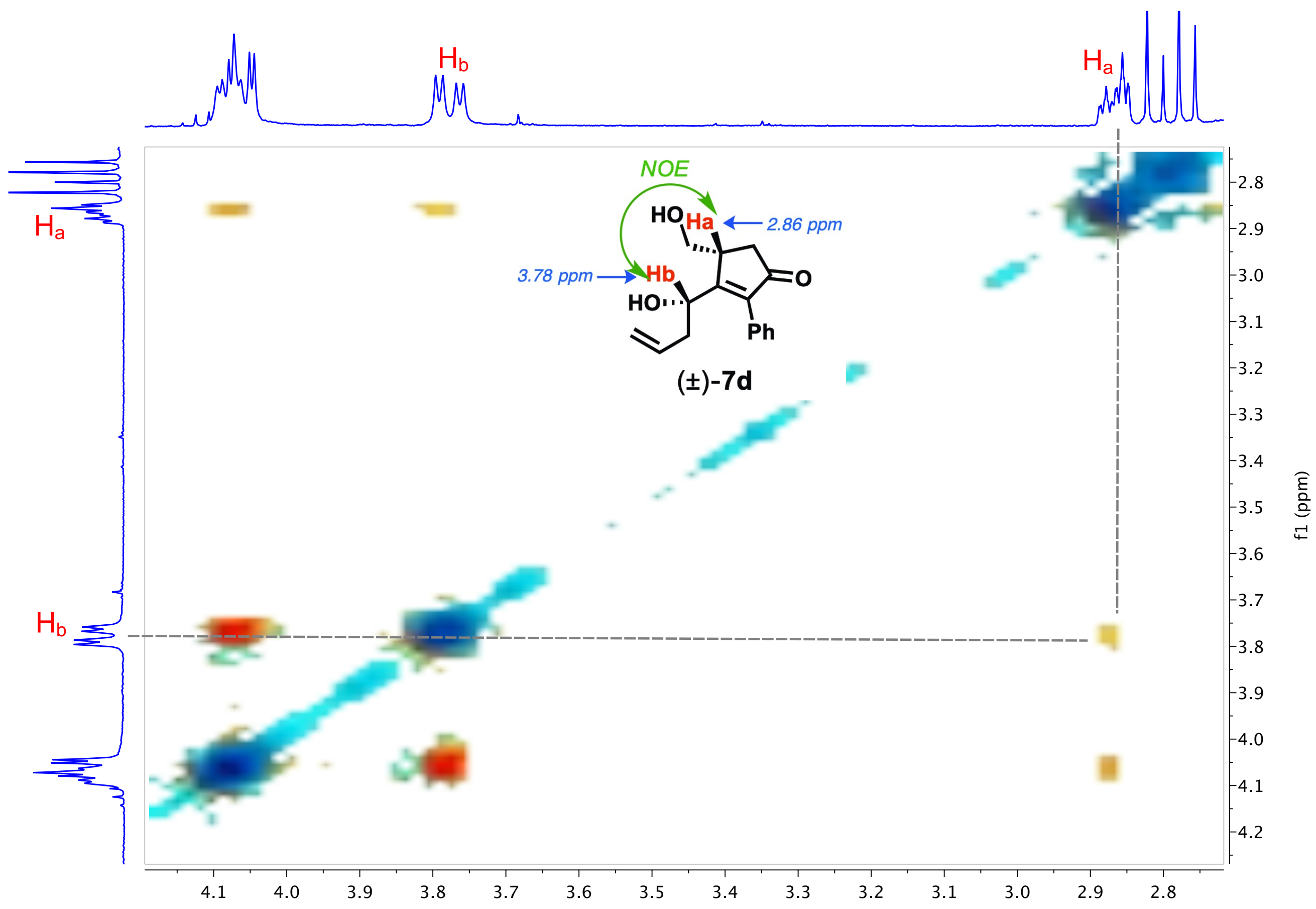




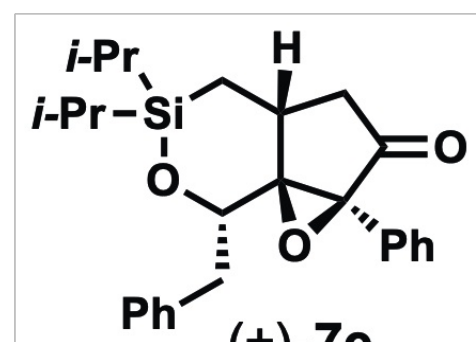

$( \pm)-7 e$

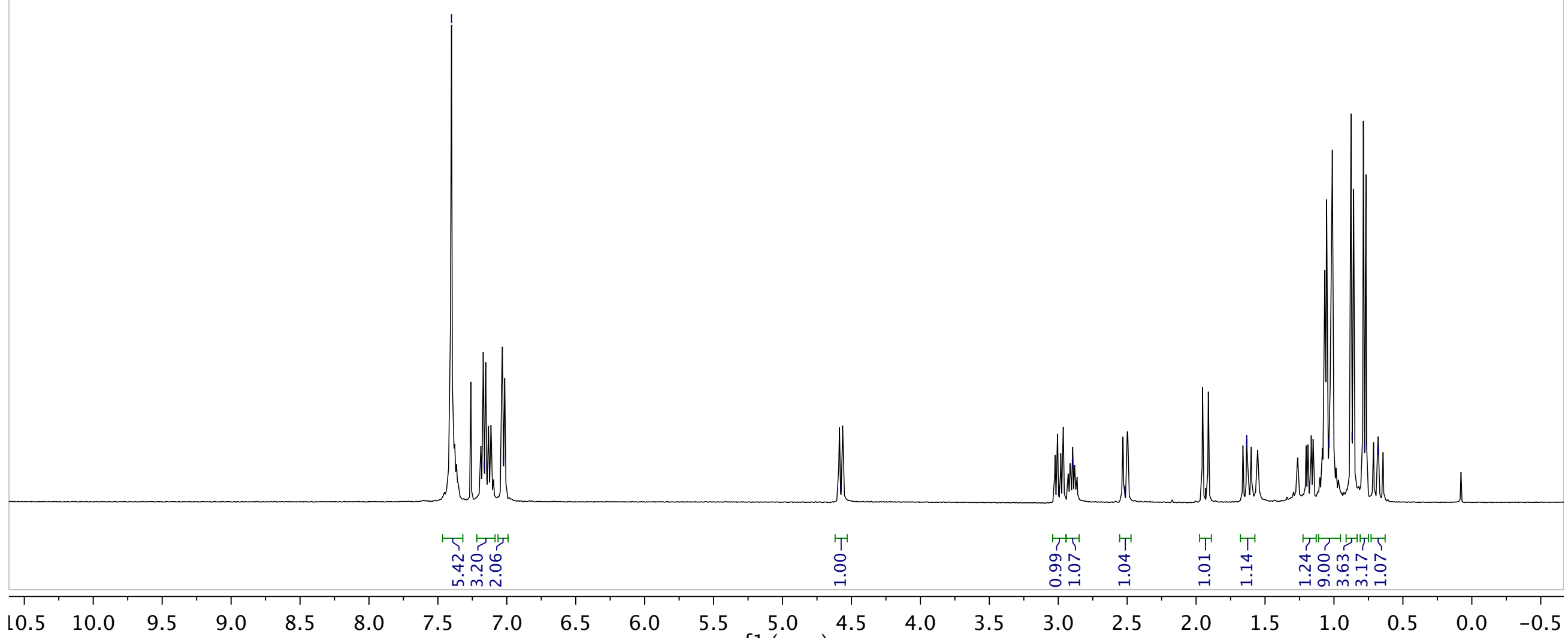




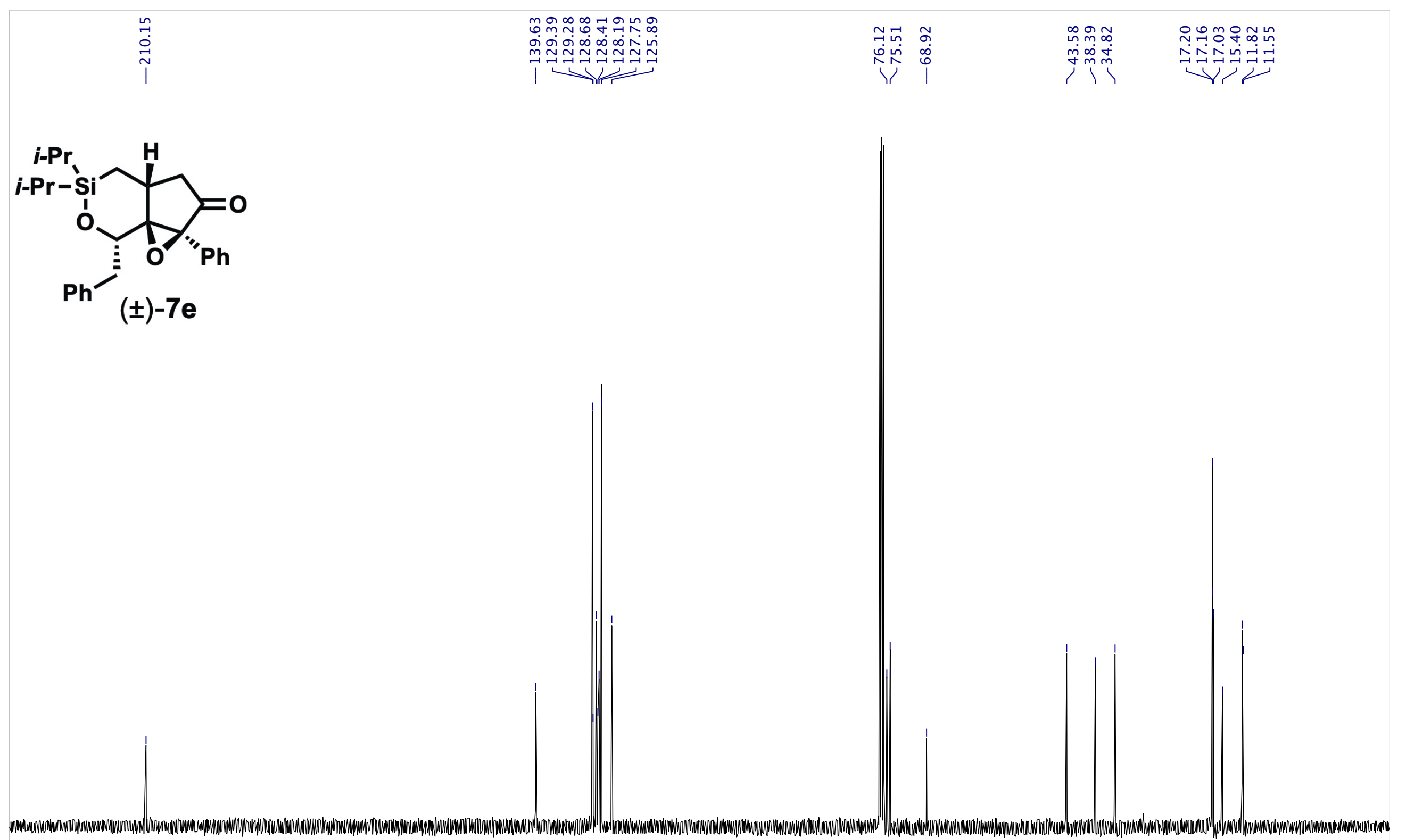

\begin{tabular}{llllllllllllllllllllllllll}
\hline 230 & 220 & 210 & 200 & 190 & 180 & 170 & 160 & 150 & 140 & 130 & 120 & 110 & 100 & 90 & 80 & 70 & 60 & 50 & 40 & 30 & 20 & 10 & 0 & -10
\end{tabular}




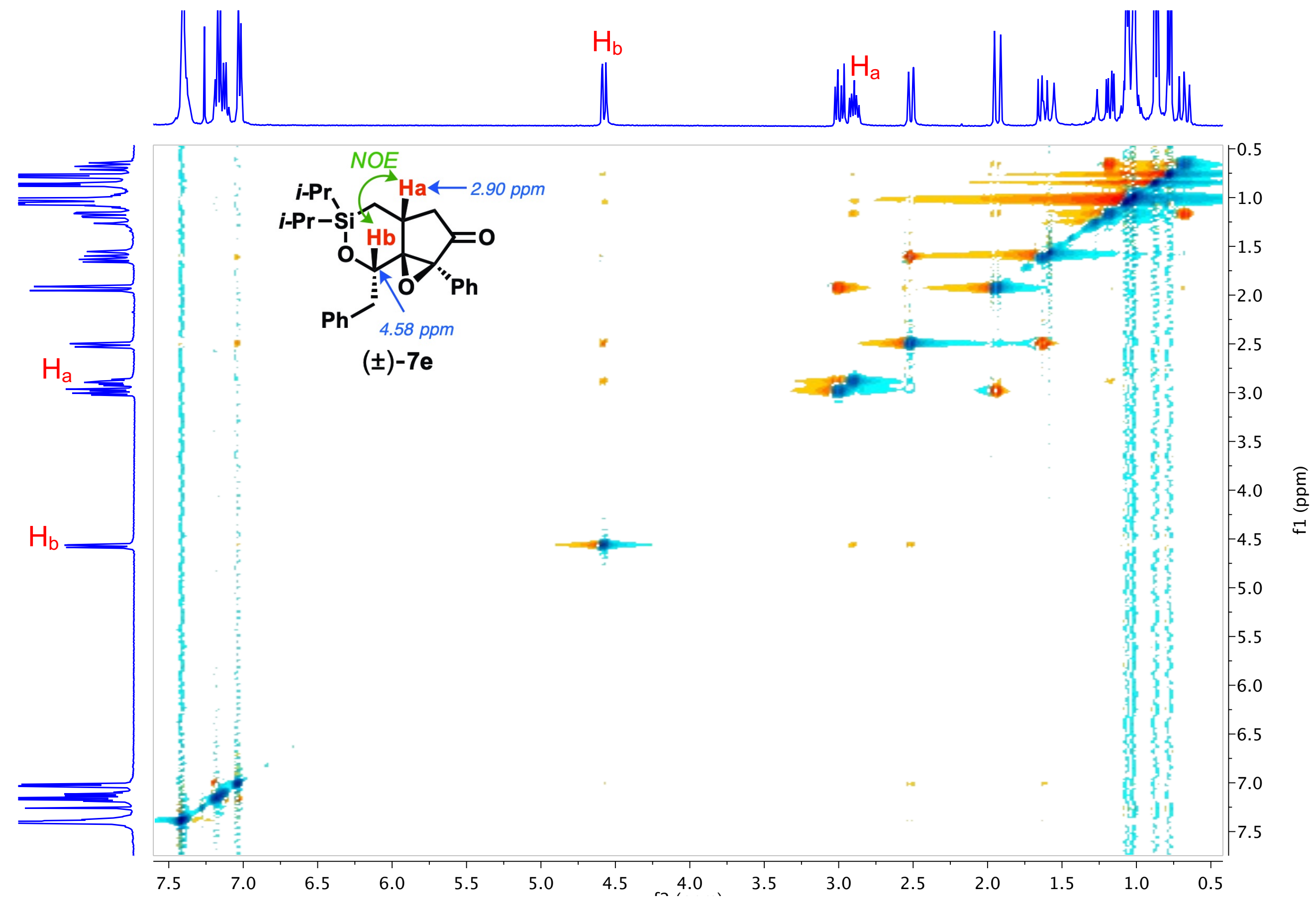




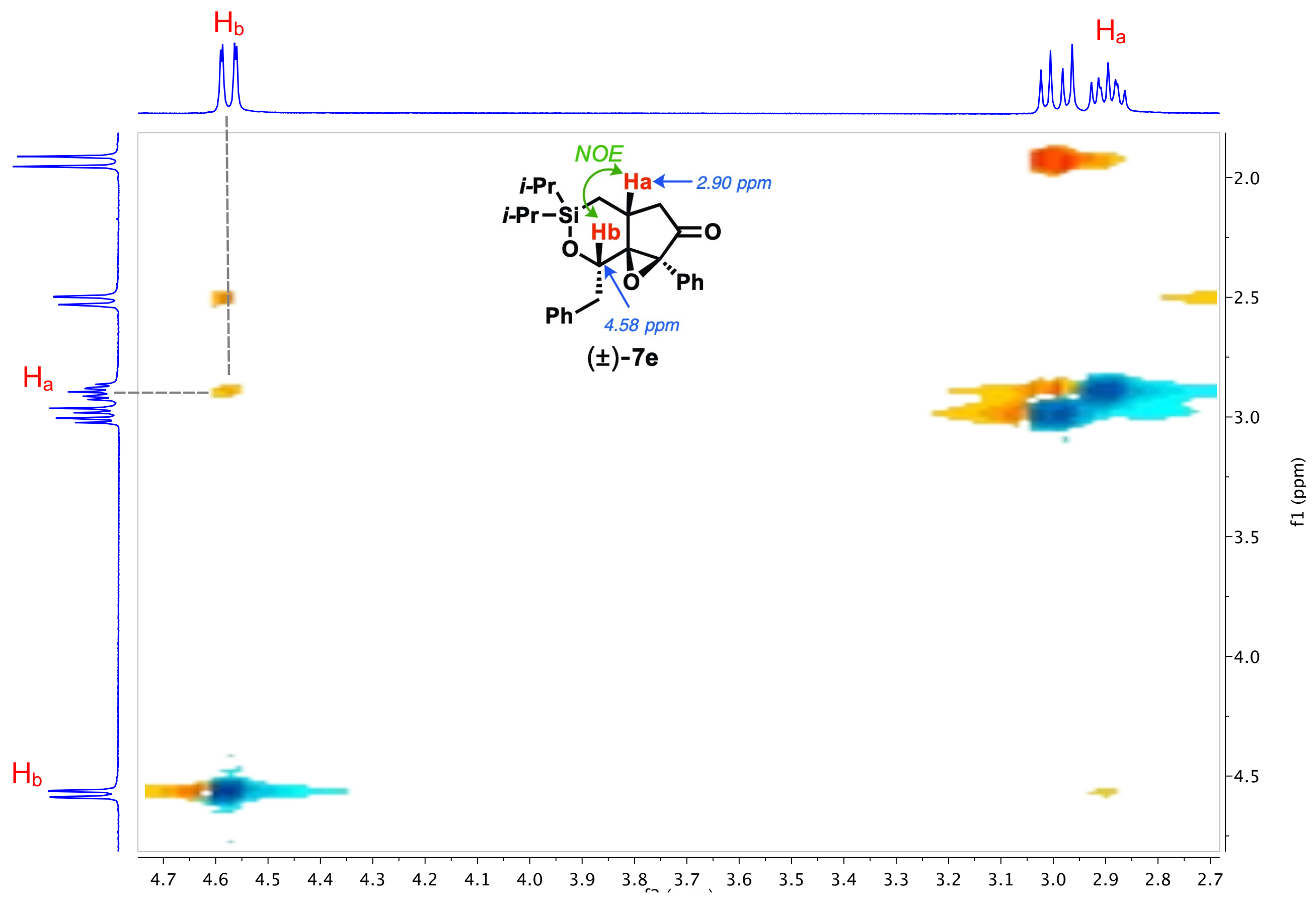




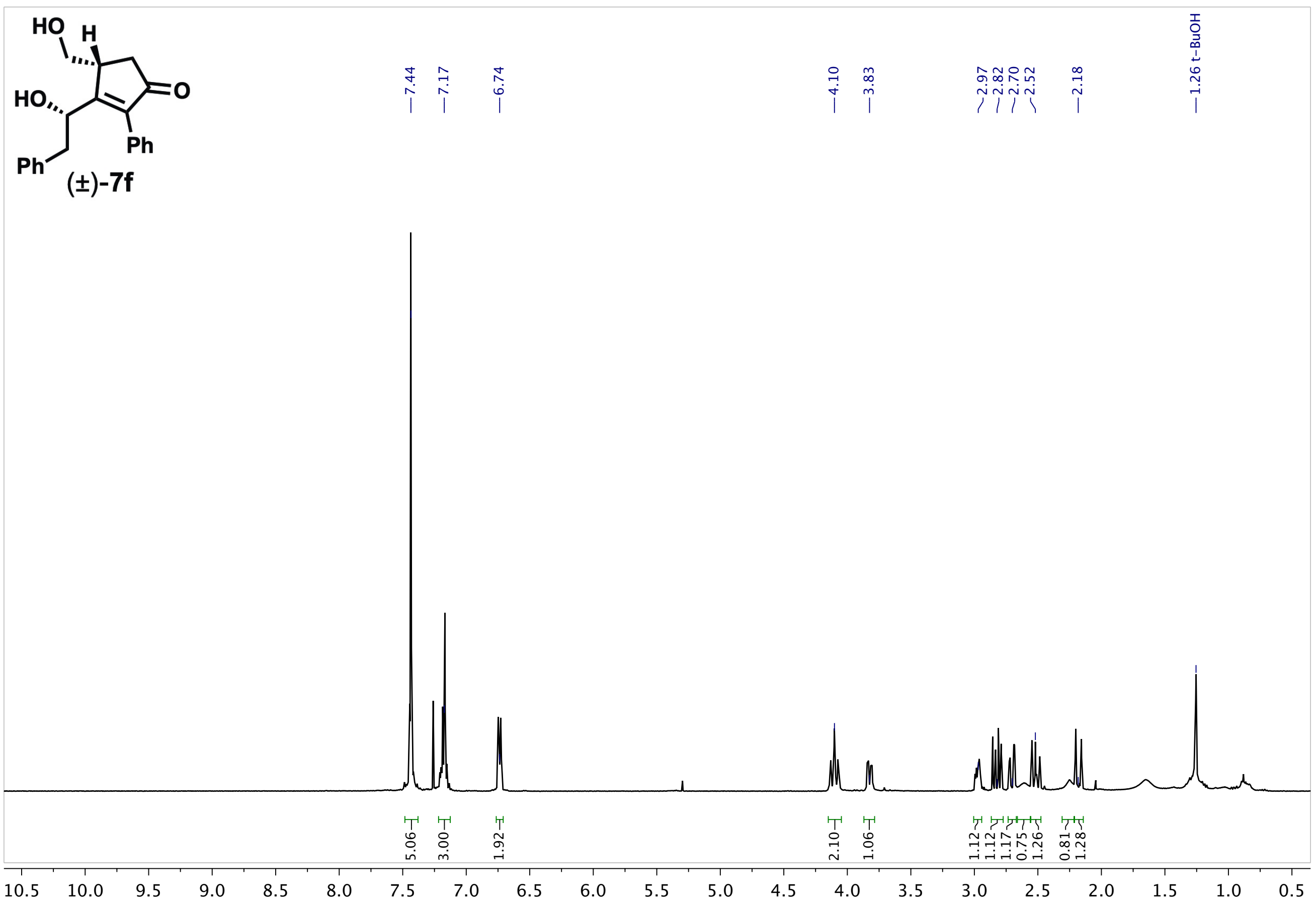


$\vec{m}$
$\infty$
$\infty$
0<smiles></smiles>

(士)-7f

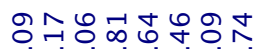

ஸ

14

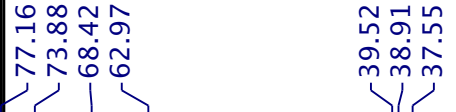

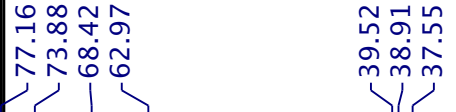

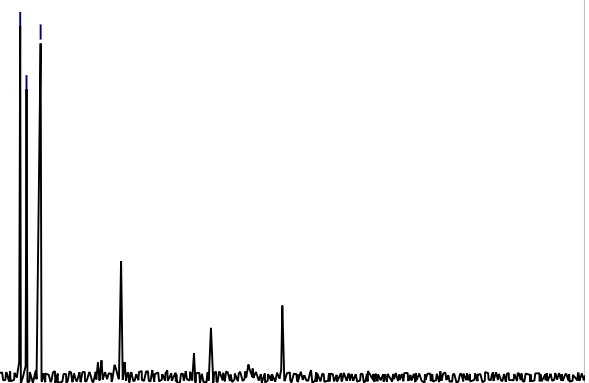




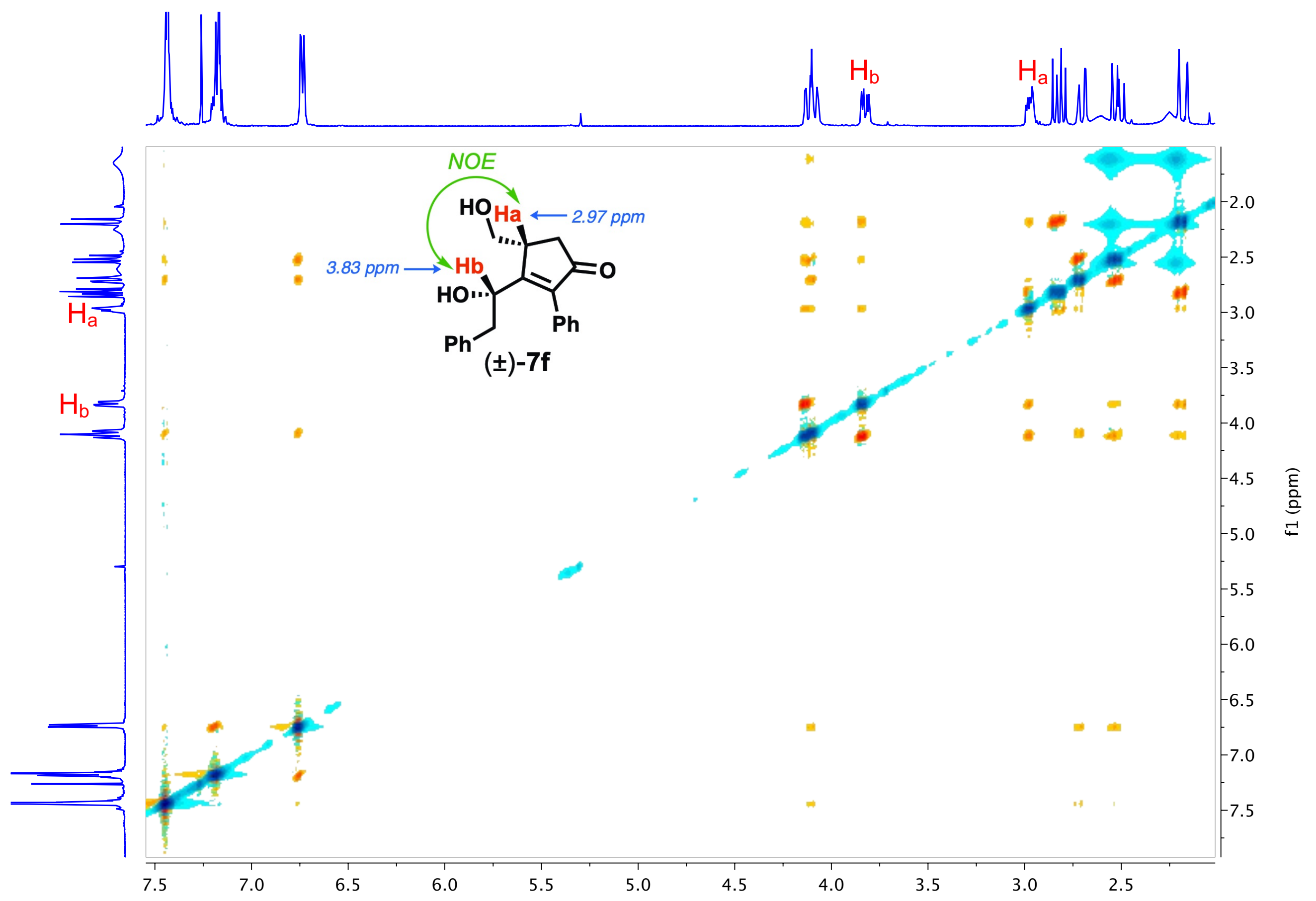




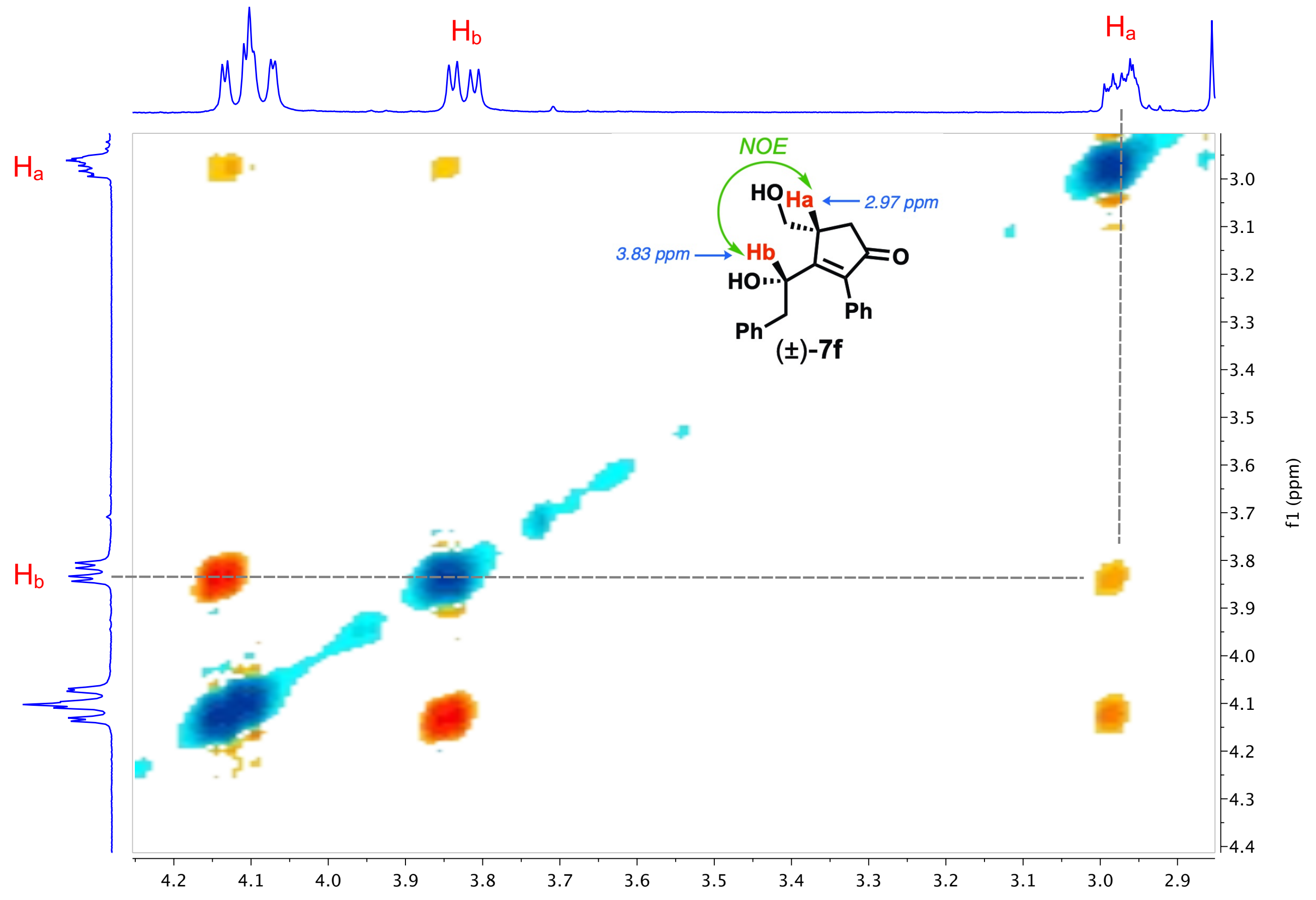


